\title{
A Radical Smiles Rearrangement Promoted by Neutral Eosin $Y$ as a Direct Hydrogen Atom Transfer Photocatalyst
}

\author{
Jianming Yan, ${ }^{\dagger}$ Han Wen Cheo, ${ }^{\dagger}$ Wei Kiat Teo, ${ }^{\dagger}$ Xiangcheng Shi, ${ }^{\dagger}$ Hui Wu, ${ }^{\ddagger}$ Shabana \\ Binte Idres, ${ }^{\ddagger}$ Lih-Wen Deng, ${ }^{\ddagger}$ Jie $\mathrm{Wu}^{*,+, \#}$ \\ 'Department of Chemistry, National University of Singapore, 3 Science Drive 3, 117543, Singapore \\ ${ }^{\ddagger}$ Department of Biochemistry, Yong Loo Lin School of Medicine, National University of Singapore, National \\ University Health System, 8 Medical Drive, 117597, Singapore \\ "National University of Singapore (Suzhou) Research Institute, No. 377 Lin Quan Street, Suzhou Industrial \\ Park, Suzhou, Jiangsu, 215123 China
}

\section{Contents}

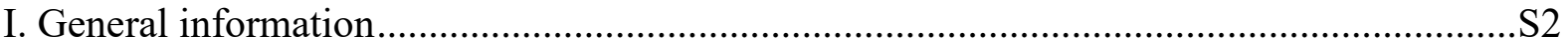

II . Biologically active compounds featuring an isothiazolidione moiety ...............................S2

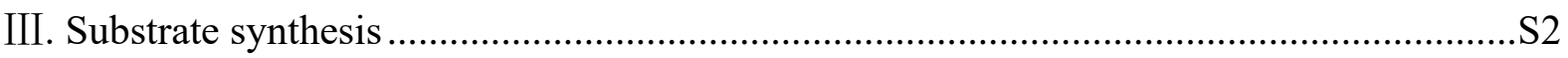

Synthesis of $N$-(hetero)arylsulfonyl propiolamide................................................................... S3

IV. Synthesis of isothiazolidin-3-one-1,1-dioxides via neutral eosin Y photocatalysis ........S11

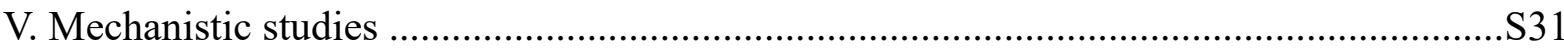

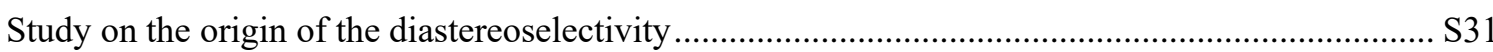

Evaluation of different modes of HAT catalysis.................................................................... S32

Control experiments indicating the involvement of vinyl radicals .............................................. S33

Evidence for the involvement of acyl radicals.......................................................................... S35

Deuterium-labelling study ....................................................................................................... S37

Determination of the quantum yield by standard ferrioxalate actinometry...................................... S41

Light on/off experiments over time …………………………………………………………….... $\mathrm{S} 42$

Luminescence quenching experiments ..................................................................................... S43

DFT calculations of SET and RHAT pathways for eosin Y regeneration ………………………….... S44

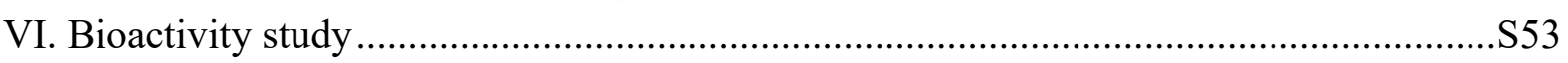

Materials and methods …………………………………………………………………….... 553

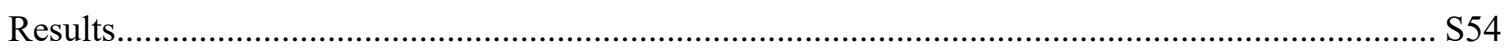

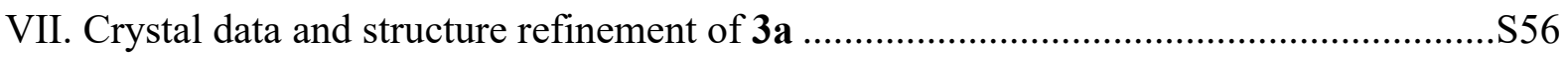

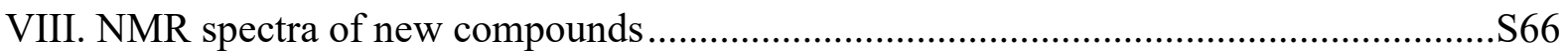

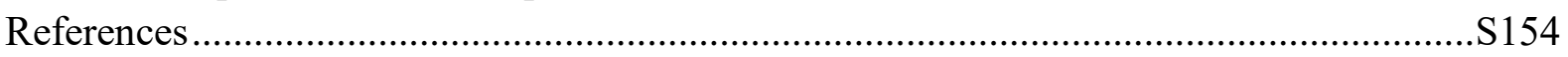




\section{General information}

Chemicals and anhydrous solvents were purchased from commercial suppliers and used as received. Commercial unavailable $N$-arylsulfonyl propiolamides were synthesized according to literature. ${ }^{1} \mathrm{H}$ NMR, ${ }^{13} \mathrm{C}$ NMR spectra were recorded on a Bruker AV-III400 (400 MHZ) or AMX500 (500 MHz) spectrometer. Chemical shifts were calibrated using residual undeuterated solvent as an internal reference $\left(\mathrm{CDCl}_{3}: 7.26 \mathrm{ppm}{ }^{1} \mathrm{H} \mathrm{NMR}, 77.16 \mathrm{ppm}{ }^{13} \mathrm{C} \mathrm{NMR}\right)$. Multiplicity was indicated as follows: s (singlet), d (doublet), $\mathrm{t}$ (triplet), q (quartet), $\mathrm{m}$ (multiplet), dd (doublet of doublet), brs (broad singlet). High-resolution mass spectra (HRMS) were obtained on a Finnigan/MAT 95XL-T spectrometer. GC analysis was performed on Aglilent 7820A \& 5977E GC-MS. HPLC analysis was performed using the corresponding commercial chiral columns (chiralcel IA, IC, ID, IE IF and OJ-H columns) as stated in the experimental procedures at $30{ }^{\circ} \mathrm{C}$ with the UV detector at $254 \mathrm{~nm}$. Cyclic voltammetry was performed using an Ametek Versa STAT 3. Blue LED strips (2 meters, $18 \mathrm{~W}$ ) were purchased from Inwares Pte Ltd (Singapore). All catalytic reactions were carried out in Schlenk tube (20 $\mathrm{mL}$ ) under an argon atmosphere with magnetic stirring after repeated freeze-pump-thaw. The isolated yield was the purified state by flash chromatography over silica gel.

\section{Biologically active compounds featuring an isothiazolidione moiety}
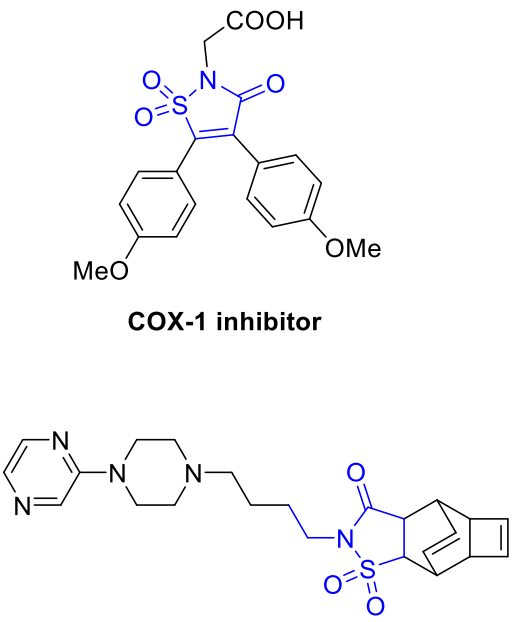

Potential anxiolytic agent
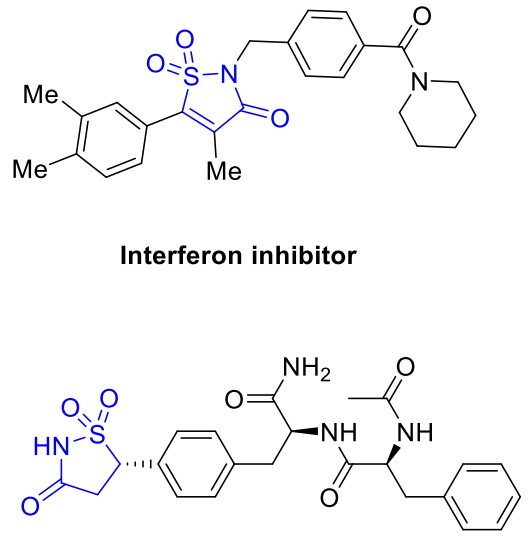

Protein Tyrosine Phosphatase inhibitor

Figure S1. Bioactive compounds featuring an isothiazolidin-3-one 1,1-dioxide moiety.

\section{Substrate synthesis}

Commercially unavailable (deuterated)aldehydes $(\mathbf{1 i}-d, \mathbf{S}-\mathbf{6 a}, \mathbf{S - 6 b}, \mathbf{S - 6 c})$ and $H$-phosphine oxides S-4 (S-4a, S-4b, S-4c, S-4d, S-4e) were prepared according to the reported procedures. Spectra data of $\mathbf{1 i}-d^{1}$ and $\mathbf{S}-\mathbf{4}^{2}$ were in line with the corresponding literature. A range of $N$ - 
(hetero)arylsulfonyl propiolamides 2 were prepared according to the literature procedure ${ }^{3}$ with slight modification (Scheme S1).

\section{Synthesis of $N$-(hetero)arylsulfonyl propiolamide}

Propiolic acid ( $0.25 \mathrm{~mL}, 4.0 \mathrm{mmol}), \mathrm{K}_{2} \mathrm{CO}_{3}(1.66 \mathrm{~g}, 12.0 \mathrm{mmol})$, THF $(20 \mathrm{~mL})$ were placed in a flask. To this mixture, pivaloyl chloride $(0.5 \mathrm{~mL}, 4.0 \mathrm{mmol})$ was added dropwise. The mixture was stirred for $3 \mathrm{~h}$ at room temperature and then the reaction mixture was filtered to remove solid impurities. The filtrate was concentrated under reduced pressure to afford crude product S-1, which was instantly used in the following step without further purification. To avoid decomposition of pivalic propiolic anhydride $\mathbf{S - 1}$, pay special attention to keep water bath temperature below $30{ }^{\circ} \mathrm{C}$ while removing solvent under rotavap.

$N$-(hetero)arylsulfonyl propiolamide $(\mathbf{S}-\mathbf{2}, 2.0 \mathrm{mmol})$ was dissolved in anhydrous THF $(10 \mathrm{~mL})$ under an inert atmosphere. The reaction mixture was cooled down to $-78{ }^{\circ} \mathrm{C}$ and $n$-BuLi $(2.0$ $\mathrm{M}$ in cyclohexane, $2.2 \mathrm{mmol}$ ) was added dropwise. The reaction was performed at $-78{ }^{\circ} \mathrm{C}$ for $1 \mathrm{~h}$ followed by slow addition of pivalic propiolic anhydride $\mathbf{S - 1}(4.0 \mathrm{mmol})$ which was freshly prepared in the first step (eq S1). Then the low temperature bath was removed and the reaction was allowed to warm up to room temperature. After the reaction was finished as judged by TLC (3-6 h), saturated $\mathrm{NH}_{4} \mathrm{Cl}$ aqueous solution $(10 \mathrm{~mL})$ was added and ethyl acetate $(3 \times 20$ $\mathrm{mL}$ ) was used for extraction. The combined organic layer was washed with brine and dried over sodium sulfate. The solvent was then removed under reduced pressure to give a crude product. Purification by silica gel column chromatography afforded the corresponding pure product 2.

\section{Scheme S1. General procedure for the synthesis of $N$-(hetero)arylsulfonyl propiolamide}<smiles>C#CC(=O)O</smiles>

$(4.0 \mathrm{mmol})$<smiles>CC(C)(C)C(=O)Cl</smiles>

$(4.0 \mathrm{mmol})$

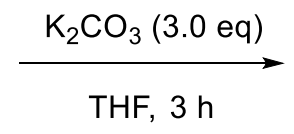

THF, $3 \mathrm{~h}$

S-1<smiles>C#CC(=O)OC(=O)C(C)(C)C</smiles>

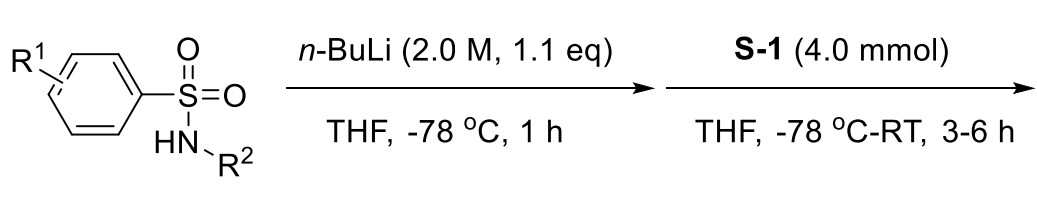

S-2 $(2.0 \mathrm{mmol})$<smiles>[R]C1C=CC(S(=O)(=O)N([R])C(=O)C#C)CC1</smiles>

2<smiles>C[C@]12CC[C@H]3[C@@H](CC[C@@H]4C[C@@H](OC(=O)c5ccc(C=O)cc5)CC[C@@]34C)[C@@H]1CCC2=O</smiles>

from epiandrosterone 
$(3 S, 5 S, 8 R, 9 S, 10 S, 13 S, 14 S)$-10,13-Dimethyl-17-oxohexadecahydro-1H-

cyclopenta $[a]$ phenanthren-3-yl 4-formylbenzoate (S-6a). Following the literature procedure, ${ }^{4}$ the title compound $(744 \mathrm{mg})$ was obtained in $88 \%$ yield starting from epiandrosterone (581 mg, $2.0 \mathrm{mmol}$ ) and 4-formyl benzoic acid (300 mg, $2.0 \mathrm{mmol}) .{ }^{1} \mathrm{H} \mathrm{NMR}$ $\left(500 \mathrm{MHz}, \mathrm{CDCl}_{3}\right) \delta 10.09(\mathrm{~s}, 1 \mathrm{H}), 8.18(\mathrm{~d}, J=8.3 \mathrm{~Hz}, 2 \mathrm{H}), 7.94(\mathrm{~d}, J=8.3 \mathrm{~Hz}, 2 \mathrm{H}), 4.97(\mathrm{tt}$, $J=11.4,4.9 \mathrm{~Hz}, 1 \mathrm{H}), 2.44(\mathrm{dd}, J=19.7,8.2 \mathrm{~Hz}, 1 \mathrm{H}), 2.07(\mathrm{dt}, J=19.3,9.0 \mathrm{~Hz}, 1 \mathrm{H}), 1.99-$ $1.90(\mathrm{~m}, 2 \mathrm{H}), 1.87-1.75(\mathrm{~m}, 4 \mathrm{H}), 1.71-1.63(\mathrm{~m}, 2 \mathrm{H}), 1.60-1.47(\mathrm{~m}, 3 \mathrm{H}), 1.41-1.22(\mathrm{~m}$, $6 \mathrm{H}), 1.12(\mathrm{td}, J=13.6,3.9 \mathrm{~Hz}, 1 \mathrm{H}), 1.05-0.96(\mathrm{~m}, 1 \mathrm{H}), 0.91(\mathrm{~s}, 3 \mathrm{H}), 0.86(\mathrm{~s}, 3 \mathrm{H}), 0.76(\mathrm{td}, J$ $=12.2,3.9 \mathrm{~Hz}, 1 \mathrm{H}) .{ }^{13} \mathrm{C} \mathrm{NMR}\left(126 \mathrm{MHz}, \mathrm{CDCl}_{3}\right) \delta 221.26,191.80,165.18,139.16,135.99$, $130.26,129.57,75.05,54.46,51.51,47.92$, 44.84, 36.86, 35.98, 35.85, 35.19, 34.12, 31.67, $30.95,28.43,27.60,21.92,20.64,13.96,12.42$. HRMS ESI $[\mathrm{M}+\mathrm{H}]^{+}$calculated for $\mathrm{C}_{27} \mathrm{H}_{35} \mathrm{O}_{4}$ 423.2530, found 423.2527 .<smiles>CC(C)[C@H]1CC[C@@H](C)C[C@H]1OC(=O)c1ccc(C=O)cc1</smiles>

from $L$-menthol

$(1 R, 2 S, 5 R)$-2-Isopropyl-5-methylcyclohexyl 4-formylbenzoate (S-6b). Following the literature procedure, ${ }^{5}$ the title compound $(519 \mathrm{mg}$ ) was obtained in $90 \%$ yield starting from $L$ menthol (313 mg, $2.0 \mathrm{mmol}$ ) and 4-formyl benzoic acid (300 mg, $2.0 \mathrm{mmol}) .{ }^{1} \mathrm{H}$ NMR (400 $\left.\mathrm{MHz}_{\mathrm{CDCl}}\right) \delta 10.09(\mathrm{~s}, 1 \mathrm{H}), 8.19(\mathrm{~d}, J=8.2 \mathrm{~Hz}, 2 \mathrm{H}), 7.94(\mathrm{~d}, J=8.4 \mathrm{~Hz}, 2 \mathrm{H}), 4.96(\mathrm{td}, J=$ $10.9,4.4 \mathrm{~Hz}, 1 \mathrm{H}), 2.18-2.08(\mathrm{~m}, 1 \mathrm{H}), 1.99-1.90(\mathrm{~m}, 1 \mathrm{H}), 1.79-1.68(\mathrm{~m}, 2 \mathrm{H}), 1.61-1.49$ (m, 2H), $1.19-1.05(\mathrm{~m}, 2 \mathrm{H}), 0.93(\mathrm{~d}, J=4.2 \mathrm{~Hz}, 3 \mathrm{H}), 0.91(\mathrm{~d}, J=4.7 \mathrm{~Hz}, 3 \mathrm{H}), 0.91-0.84$ $(\mathrm{m}, 1 \mathrm{H}), 0.79$ (d, $J=7.0 \mathrm{~Hz}, 3 \mathrm{H}) .{ }^{13} \mathrm{C} \mathrm{NMR}\left(126 \mathrm{MHz}, \mathrm{CDCl}_{3}\right) \delta 191.78,165.16,139.13$, $135.95,130.26,129.59,75.78,47.34,41.00,34.36,31.57,26.67,23.73,22.14,20.87,16.62$. HRMS ESI $[\mathrm{M}+\mathrm{H}]^{+}$calculated for $\mathrm{C}_{18} \mathrm{H}_{25} \mathrm{O}_{3} 289.1798$, found 289.1788 .

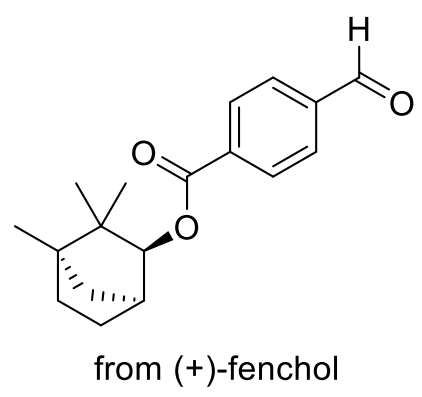

(1S,2S,4R)-3,3,4-Trimethylbicyclo[2.2.1]heptan-2-yl 4-formylbenzoate (S-6c). Following the literature procedure, ${ }^{6}$ the title compound $(458 \mathrm{mg}$ ) was obtained in $80 \%$ yield starting from (+)-fenchol (309 mg, $2.0 \mathrm{mmol}$ ) and 4-formyl benzoic acid (300 mg, $2.0 \mathrm{mmol}) .{ }^{1} \mathrm{H} \mathrm{NMR}(400$ $\left.\mathrm{MHz}, \mathrm{CDCl}_{3}\right) \delta 10.11(\mathrm{~s}, 1 \mathrm{H}), 8.21(\mathrm{~d}, J=8.1 \mathrm{~Hz}, 2 \mathrm{H}), 7.96(\mathrm{~d}, J=8.6 \mathrm{~Hz}, 2 \mathrm{H}), 4.65(\mathrm{~d}, J=$ $1.9 \mathrm{~Hz}, 1 \mathrm{H}), 1.92$ (dddd, $J=12.3,8.9,5.7,2.2 \mathrm{~Hz}, 1 \mathrm{H}), 1.83-1.75(\mathrm{~m}, 2 \mathrm{H}), 1.67$ (dq, $J=10.4$, $2.2 \mathrm{~Hz}, 1 \mathrm{H}), 1.59-1.48(\mathrm{~m}, 1 \mathrm{H}), 1.31-1.21(\mathrm{~m}, 2 \mathrm{H}), 1.19$ (s, 3H), $1.12(\mathrm{~s}, 3 \mathrm{H}), 0.85(\mathrm{~s}, 3 \mathrm{H})$. 
${ }^{13} \mathrm{C} \mathrm{NMR}\left(126 \mathrm{MHz}, \mathrm{CDCl}_{3}\right) \delta 191.78,165.99,139.22,135.80,130.23,129.70,87.62,48.79$, 48.53, 41.59, 40.03, 29.89, 27.02, 26.03, 20.43, 19.62. HRMS ESI $[\mathrm{M}+\mathrm{H}]^{+}$calculated for $\mathrm{C}_{18} \mathrm{H}_{23} \mathrm{O}_{3} 287.1642$, found 287.1638 .<smiles>C#CC(=O)N(C)S(=O)(=O)c1ccc(C)cc1</smiles>

$N$-methyl- $N$-tosylpropiolamide (2a). Following the literature procedure, ${ }^{3}$ the title compound $(281 \mathrm{mg}$ ) was obtained in 59\% yield starting from $N$,4-dimethylbenzenesulfonamide $(370 \mathrm{mg}$, $2.0 \mathrm{mmol}) .{ }^{1} \mathrm{H} \mathrm{NMR}\left(400 \mathrm{MHz}, \mathrm{CDCl}_{3}\right) \delta 7.88(\mathrm{~d}, J=8.0 \mathrm{~Hz}, 2 \mathrm{H}), 7.34(\mathrm{~d}, J=8.0 \mathrm{~Hz}, 2 \mathrm{H})$, $3.45(\mathrm{~s}, 3 \mathrm{H}), 3.31(\mathrm{~s}, 1 \mathrm{H}), 2.45(\mathrm{~s}, 3 \mathrm{H}) .{ }^{13} \mathrm{C} \mathrm{NMR}\left(101 \mathrm{MHz}, \mathrm{CDCl}_{3}\right) \delta 151.80,145.62,135.37$, 129.87, 128.48, 82.29, 75.37, 33.57, 21.84. HRMS ESI $[\mathrm{M}+\mathrm{H}]^{+}$calculated for $\mathrm{C}_{11} \mathrm{H}_{12} \mathrm{NO}_{3} \mathrm{~S}$ 238.0532, found 238.0527 .<smiles>C#CC(=O)N(C)S(=O)(=O)c1ccccc1</smiles>

$N$-methyl- $N$-(phenylsulfonyl)propiolamide (2b). Following the literature procedure, ${ }^{3}$ the title compound $(255 \mathrm{mg}$ ) was obtained in $57 \%$ yield starting from $\mathrm{N}$-methylbenzenesulfonamide (342 mg, $2.0 \mathrm{mmol}) .{ }^{1} \mathrm{H}$ NMR $\left(500 \mathrm{MHz}, \mathrm{CDCl}_{3}\right) \delta 8.02-7.96(\mathrm{~m}, 2 \mathrm{H}), 7.66(\mathrm{t}, J=7.5 \mathrm{~Hz}$, $1 \mathrm{H}), 7.58-7.51(\mathrm{~m}, 2 \mathrm{H}), 3.46(\mathrm{~s}, 3 \mathrm{H}), 3.35(\mathrm{~s}, 1 \mathrm{H}) .{ }^{13} \mathrm{C} \mathrm{NMR}\left(126 \mathrm{MHz}, \mathrm{CDCl}_{3}\right) \delta 151.75$, $138.19,134.37,129.22,128.34,82.57,75.18,33.65$. HRMS ESI $[\mathrm{M}+\mathrm{H}]^{+}$calculated for $\mathrm{C}_{10} \mathrm{H}_{10} \mathrm{NO}_{3} \mathrm{~S} 224.0376$, found 224.0369.<smiles>C#CC(=O)N(C)S(=O)(=O)c1ccc(OC)cc1</smiles>

$N$-((4-methoxyphenyl)sulfonyl)- $N$-methylpropiolamide (2d). Following the literature procedure, ${ }^{3}$ the title compound (294 mg) was obtained in 58\% yield starting from 4-methoxy$N$-methylbenzenesulfonamide (402 mg, $2.0 \mathrm{mmol}) .{ }^{1} \mathrm{H} \mathrm{NMR}\left(500 \mathrm{MHz}, \mathrm{CDCl}_{3}\right) \delta 7.94(\mathrm{~d}, J=$ $8.7 \mathrm{~Hz}, 2 \mathrm{H}), 7.00(\mathrm{~d}, J=8.9 \mathrm{~Hz}, 2 \mathrm{H}), 3.88(\mathrm{~s}, 3 \mathrm{H}), 3.45(\mathrm{~s}, 3 \mathrm{H}), 3.31(\mathrm{~s}, 1 \mathrm{H}) .{ }^{13} \mathrm{C} \mathrm{NMR}(126$ $\left.\mathrm{MHz}, \mathrm{CDCl}_{3}\right) \delta 164.31,151.82,130.86,129.62,114.40,82.15,75.41,55.90,33.62 . \mathrm{HRMS}$ ESI $[\mathrm{M}+\mathrm{H}]^{+}$calculated for $\mathrm{C}_{11} \mathrm{H}_{12} \mathrm{NO}_{4} \mathrm{~S} 254.0482$, found 254.0480 .<smiles>C#CC(=O)N(C)S(=O)(=O)c1ccc(CC(C)C)cc1</smiles>

$N$-((4-(tert-butyl)phenyl)sulfonyl)- $N$-methylpropiolamide (2e). Following the literature procedure, ${ }^{3}$ the title compound $(285 \mathrm{mg}$ ) was obtained in $51 \%$ yield starting from 4-(tert-butyl)$N$-methylbenzenesulfonamide $(454 \mathrm{mg}, 2.0 \mathrm{mmol}) .{ }^{1} \mathrm{H} \mathrm{NMR}\left(500 \mathrm{MHz}, \mathrm{CDCl}_{3}\right) \delta 7.92(\mathrm{~d}, J=$ 
$8.5 \mathrm{~Hz}, 2 \mathrm{H}), 7.55(\mathrm{~d}, J=8.7 \mathrm{~Hz}, 2 \mathrm{H}), 3.47(\mathrm{~s}, 3 \mathrm{H}), 3.33(\mathrm{~s}, 1 \mathrm{H}), 1.35$ (s, 9H). ${ }^{13} \mathrm{C}$ NMR $(126$ $\left.\mathrm{MHz}, \mathrm{CDCl}_{3}\right) \delta 158.49,151.83,135.20,128.32,126.31,82.31,75.43,35.50,33.59,31.15$. HRMS ESI $[\mathrm{M}+\mathrm{H}]^{+}$calculated for $\mathrm{C}_{14} \mathrm{H}_{18} \mathrm{NO}_{3} \mathrm{~S} 280.1002$, found 280.1004 .<smiles>C#CC(=O)N(C)S(=O)(=O)c1ccc(NC(C)C)cc1</smiles>

$N$-((4-acetamidophenyl)sulfonyl)- $N$-methylpropiolamide (2f). Following the literature procedure, ${ }^{3}$ the title compound $(224 \mathrm{mg})$ was obtained in $40 \%$ yield starting from $\mathrm{N}-(4-(\mathrm{N}-$ methylsulfamoyl)phenyl)acetamide $(457 \mathrm{mg}, 2.0 \mathrm{mmol}) .{ }^{1} \mathrm{H}$ NMR (500 MHz, Acetone- $\left.d_{6}\right) \delta$ $9.64(\mathrm{~s}, 1 \mathrm{H}), 7.96(\mathrm{~d}, J=8.9 \mathrm{~Hz}, 2 \mathrm{H}), 7.88(\mathrm{~d}, J=9.0 \mathrm{~Hz}, 2 \mathrm{H}), 4.26(\mathrm{~s}, 1 \mathrm{H}), 3.49(\mathrm{~s}, 3 \mathrm{H}), 2.14$ $(\mathrm{s}, 3 \mathrm{H}) .{ }^{13} \mathrm{C}$ NMR $(126 \mathrm{MHz}$, Acetone) $\delta 169.73,152.41,145.67,132.79,130.51,119.26,84.30$, 76.06, 34.00, 24.43. HRMS ESI $[\mathrm{M}+\mathrm{H}]^{+}$calculated for $\mathrm{C}_{12} \mathrm{H}_{13} \mathrm{~N}_{2} \mathrm{O}_{4} \mathrm{~S}$ 281.0591, found 281.0589 .

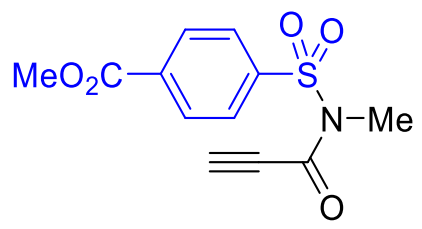

Methyl 4-( $N$-methyl- $N$-propioloylsulfamoyl)benzoate (2g). Following the literature procedure, ${ }^{3}$ the title compound $(259 \mathrm{mg})$ was obtained in $46 \%$ yield starting from methyl $4-(\mathrm{N}$ methylsulfamoyl)benzoate $(458 \mathrm{mg}, 2.0 \mathrm{mmol}) .{ }^{1} \mathrm{H} \mathrm{NMR}\left(500 \mathrm{MHz}, \mathrm{CDCl}_{3}\right) \delta 8.20(\mathrm{~d}, J=8.6$ $\mathrm{Hz}, 2 \mathrm{H}), 8.07(\mathrm{~d}, J=8.7 \mathrm{~Hz}, 2 \mathrm{H}), 3.97(\mathrm{~s}, 3 \mathrm{H}), 3.51(\mathrm{~s}, 3 \mathrm{H}), 3.34(\mathrm{~s}, 1 \mathrm{H}) .{ }^{13} \mathrm{C}$ NMR $(126 \mathrm{MHz}$, $\left.\mathrm{CDCl}_{3}\right) \delta 165.42,151.73,141.99,135.35,130.36,128.54,82.74,75.07,52.94,33.85$. HRMS ESI $[\mathrm{M}+\mathrm{H}]^{+}$calculated for $\mathrm{C}_{12} \mathrm{H}_{12} \mathrm{NO}_{5} \mathrm{~S} 282.0431$, found 282.0426 .<smiles>C#CC(=O)N(C)S(=O)(=O)c1ccc(C(F)(F)F)cc1</smiles>

$N$-methyl- $N$-((4-(trifluoromethyl)phenyl)sulfonyl)propiolamide (2h). Following the literature procedure, ${ }^{3}$ the title compound $(245 \mathrm{mg}$ ) was obtained in $42 \%$ yield starting from $\mathrm{N}$ methyl-4-(trifluoromethyl)benzenesulfonamide (478 mg, $2.0 \mathrm{mmol}) .{ }^{1} \mathrm{H}$ NMR $(500 \mathrm{MHz}$, $\left.\mathrm{CDCl}_{3}\right) \delta 8.14(\mathrm{~d}, J=8.0 \mathrm{~Hz}, 2 \mathrm{H}), 7.82(\mathrm{~d}, J=8.1 \mathrm{~Hz}, 2 \mathrm{H}), 3.53(\mathrm{~s}, 3 \mathrm{H}), 3.35(\mathrm{~s}, 1 \mathrm{H}) .{ }^{13} \mathrm{C} \mathrm{NMR}$ $\left(126 \mathrm{MHz}, \mathrm{CDCl}_{3}\right) \delta 151.76,141.72,135.96\left(\mathrm{q},{ }^{2} J_{\mathrm{C}-\mathrm{F}}=34.0 \mathrm{~Hz}\right), 129.19,126.41\left(\mathrm{q},{ }^{3} J_{\mathrm{C}-\mathrm{F}}=\right.$ $3.8 \mathrm{~Hz}), 123.13\left(\mathrm{q},{ }^{1} J_{\mathrm{C}-\mathrm{F}}=34.0 \mathrm{~Hz}\right), 82.79,75.01,34.00$. HRMS ESI $[\mathrm{M}+\mathrm{H}]^{+}$calculated for $\mathrm{C}_{11} \mathrm{H}_{9} \mathrm{~F}_{3} \mathrm{NO}_{3} \mathrm{~S} 292.0250$, found 292.0245.<smiles>C#CC(=O)N(C)S(=O)(=O)c1ccc(F)cc1</smiles> 
$N$-((4-fluorophenyl)sulfonyl)- $N$-methylpropiolamide (2i). Following the literature procedure, ${ }^{3}$ the title compound $(275 \mathrm{mg}$ ) was obtained in $57 \%$ yield starting from 4-fluoro- $N$ methylbenzenesulfonamide (378 mg, $2.0 \mathrm{mmol}) .{ }^{1} \mathrm{H} \mathrm{NMR}\left(500 \mathrm{MHz}, \mathrm{CDCl}_{3}\right) \delta 8.07$ (dd, $J=$ 8.0, 5.0 Hz, 2H), 7.27 (dd, $J=16.9,8.0 \mathrm{~Hz}, 2 \mathrm{H}), 3.52(\mathrm{~s}, 3 \mathrm{H}), 3.36(\mathrm{~s}, 1 \mathrm{H}) .{ }^{13} \mathrm{C} \mathrm{NMR}(126$ $\left.\mathrm{MHz}, \mathrm{CDCl}_{3}\right) \delta 166.19\left(\mathrm{~d},{ }^{1} J_{\mathrm{C}-\mathrm{F}}=258.2 \mathrm{~Hz}\right), 151.77,134.27,131.57\left(\mathrm{~d},{ }^{3} J_{\mathrm{C}-\mathrm{F}}=9.7 \mathrm{~Hz}\right)$, $116.58\left(\mathrm{~d},{ }^{2} J_{\mathrm{C}-\mathrm{F}}=22.9 \mathrm{~Hz}\right), 82.44,75.20,33.83 .{ }^{19} \mathrm{~F}$ NMR $\left(376 \mathrm{MHz}, \mathrm{CDCl}_{3}\right) \delta-102.00$. HRMS ESI $[\mathrm{M}+\mathrm{H}]^{+}$calculated for $\mathrm{C}_{10} \mathrm{H}_{9} \mathrm{FNO}_{3} \mathrm{~S} 242.0282$, found 242.0278.

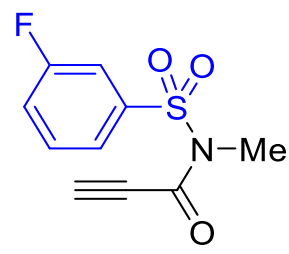

$N$-((3-fluorophenyl)sulfonyl)- $N$-methylpropiolamide (2j). Following the literature procedure, ${ }^{3}$ the title compound $(217 \mathrm{mg}$ ) was obtained in $45 \%$ yield starting from 3-fluoro- $\mathrm{N}$ methylbenzenesulfonamide $(378 \mathrm{mg}, 2.0 \mathrm{mmol}) .{ }^{1} \mathrm{H}$ NMR $\left(500 \mathrm{MHz}, \mathrm{CDCl}_{3}\right) \delta 7.79$ (ddd, $J=$ 7.9, 1.8, $1.0 \mathrm{~Hz}, 1 \mathrm{H}), 7.71(\mathrm{dt}, J=8.1,2.1 \mathrm{~Hz}, 1 \mathrm{H}), 7.55(\mathrm{td}, J=8.1,5.2 \mathrm{~Hz}, 1 \mathrm{H}), 7.37$ (tdd, $J$ $=8.2,2.6,0.9 \mathrm{~Hz}, 1 \mathrm{H}), 3.48(\mathrm{~s}, 3 \mathrm{H}), 3.37(\mathrm{~s}, 1 \mathrm{H}) .{ }^{13} \mathrm{C} \mathrm{NMR}\left(126 \mathrm{MHz}, \mathrm{CDCl}_{3}\right) \delta 163.26\left(\mathrm{~d},{ }^{1} \mathrm{~J}\right.$ $\mathrm{C}-\mathrm{F}=252.6 \mathrm{~Hz}), 151.70,140.09\left(\mathrm{~d},{ }^{3} J_{\mathrm{C}-\mathrm{F}}=7.3 \mathrm{~Hz}\right), 131.07\left(\mathrm{~d},{ }^{3} J_{\mathrm{C}-\mathrm{F}}=7.8 \mathrm{~Hz}\right), 124.23\left(\mathrm{~d},{ }^{4} J\right.$ $\mathrm{C}-\mathrm{F}=3.4 \mathrm{~Hz}), 121.69\left(\mathrm{~d},{ }^{2} J_{\mathrm{C}-\mathrm{F}}=21.3 \mathrm{~Hz}\right), 115.90\left(\mathrm{~d},{ }^{2} J_{\mathrm{C}-\mathrm{F}}=24.9 \mathrm{~Hz}\right), 82.75,75.08,33.82$. ${ }^{19} \mathrm{~F}$ NMR $\left(376 \mathrm{MHz}, \mathrm{CDCl}_{3}\right) \delta-108.89$. HRMS ESI $[\mathrm{M}+\mathrm{H}]^{+}$calculated for $\mathrm{C}_{10} \mathrm{H}_{9} \mathrm{FNO}_{3} \mathrm{~S}$ 242.0282 , found 242.0285 .<smiles>C#CC(=O)N(C)S(=O)(=O)c1cccc(OC)c1</smiles>

$N$-((3-methoxyphenyl)sulfonyl)- $N$-methylpropiolamide (2k). Following the literature procedure, ${ }^{3}$ the title compound $(243 \mathrm{mg}$ ) was obtained in $48 \%$ yield starting from 3-methoxy$\mathrm{N}$-methylbenzenesulfonamide (402 mg, $2.0 \mathrm{mmol}$ ). ${ }^{1} \mathrm{H} \mathrm{NMR}\left(400 \mathrm{MHz}, \mathrm{CDCl}_{3}\right) \delta 7.57-7.50$ (m, 2H), 7.45 (t, $J=8.0 \mathrm{~Hz}, 1 \mathrm{H}), 7.18(\mathrm{ddd}, J=8.3,2.6,1.0 \mathrm{~Hz}, 1 \mathrm{H}), 3.87(\mathrm{~s}, 3 \mathrm{H}), 3.45(\mathrm{~s}, 3 \mathrm{H})$, 3.35 (s, 1H). ${ }^{13} \mathrm{C}$ NMR $\left(126 \mathrm{MHz}, \mathrm{CDCl}_{3}\right) \delta 159.97,151.76,139.36,130.32,120.73,120.27$, 113.22, 82.46, 75.35, 55.89, 33.62. HRMS ESI $[\mathrm{M}+\mathrm{H}]^{+}$calculated for $\mathrm{C}_{11} \mathrm{H}_{12} \mathrm{NO}_{4} \mathrm{~S} 254.0482$, found 254.0485 .

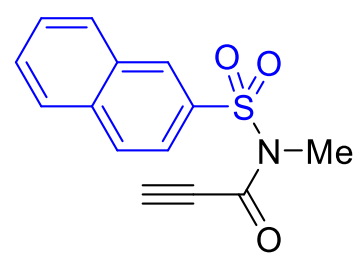

$N$-methyl- $N$-(naphthalen-2-ylsulfonyl)propiolamide (2l). Following the literature procedure, ${ }^{3}$ the title compound $(317 \mathrm{mg})$ was obtained in $58 \%$ yield starting from $\mathrm{N}$ methylnaphthalene-2-sulfonamide (443 mg, $2.0 \mathrm{mmol}) .{ }^{1} \mathrm{H}$ NMR $\left(500 \mathrm{MHz}, \mathrm{CDCl}_{3}\right) \delta 8.60$ (d, $J=2.0 \mathrm{~Hz}, 1 \mathrm{H}), 8.00(\mathrm{t}, J=9.2 \mathrm{~Hz}, 2 \mathrm{H}), 7.95-7.90(\mathrm{~m}, 2 \mathrm{H}), 7.69(\mathrm{ddd}, J=8.2,6.9,1.3 \mathrm{~Hz}$, 
1H), $7.64(\mathrm{ddd}, J=8.2,6.9,1.3 \mathrm{~Hz}, 1 \mathrm{H}), 3.53(\mathrm{~s}, 3 \mathrm{H}), 3.33(\mathrm{~s}, 1 \mathrm{H}) .{ }^{13} \mathrm{C}$ NMR $(126 \mathrm{MHz}$, $\left.\mathrm{CDCl}_{3}\right) \delta 151.80,135.63,135.02,131.96,130.81,129.81,129.73,129.64,128.12,128.02$, 122.57, 82.42, 75.34, 33.77. HRMS ESI $[\mathrm{M}+\mathrm{H}]^{+}$calculated for $\mathrm{C}_{14} \mathrm{H}_{12} \mathrm{NO}_{3} \mathrm{~S} 274.0532$, found 274.0527 .<smiles>C#CC(=O)N(C)S(=O)(=O)c1ccccc1OC</smiles>

$N$-((2-methoxyphenyl)sulfonyl)- $N$-methylpropiolamide (2m). Following the literature procedure, ${ }^{3}$ the title compound $(269 \mathrm{mg}$ ) was obtained in 53\% yield starting from 2-methoxy$N$-methylbenzenesulfonamide (402 mg, $2.0 \mathrm{mmol}) .{ }^{1} \mathrm{H}$ NMR $\left(500 \mathrm{MHz}, \mathrm{CDCl}_{3}\right) \delta 8.06$ (dd, $J$ $=8.0,1.7 \mathrm{~Hz}, 1 \mathrm{H}), 7.60(\mathrm{ddd}, J=8.4,7.4,1.7 \mathrm{~Hz}, 1 \mathrm{H}), 7.10(\mathrm{ddd}, J=8.2,7.4,1.0 \mathrm{~Hz}, 1 \mathrm{H})$, $7.02(\mathrm{dd}, J=8.4,0.9 \mathrm{~Hz}, 1 \mathrm{H}), 3.95(\mathrm{~s}, 3 \mathrm{H}), 3.43(\mathrm{~s}, 3 \mathrm{H}), 3.22(\mathrm{~s}, 1 \mathrm{H}) .{ }^{13} \mathrm{C}$ NMR $(126 \mathrm{MHz}$, $\left.\mathrm{CDCl}_{3}\right) \delta 157.09,152.02,136.19,132.51,126.28,120.49,112.43,82.24,75.47,56.41$. HRMS ESI $[\mathrm{M}+\mathrm{H}]^{+}$calculated for $\mathrm{C}_{11} \mathrm{H}_{12} \mathrm{NO}_{4} \mathrm{~S} 254.0482$, found 254.0487 .<smiles>C#CC(=O)N(C)S(=O)(=O)c1cccs1</smiles>

$N$-methyl- $N$-(thiophen-2-ylsulfonyl)propiolamide (2n). Following the literature procedure, the title compound $(252 \mathrm{mg}$ ) was obtained in 55\% yield starting from $\mathrm{N}$-methylthiophene-2sulfonamide (354 mg, $2.0 \mathrm{mmol}) .{ }^{1} \mathrm{H}$ NMR $\left(500 \mathrm{MHz}, \mathrm{CDCl}_{3}\right) \delta 7.87(\mathrm{dd}, J=3.8,1.4 \mathrm{~Hz}, 1 \mathrm{H})$, $7.72(\mathrm{dd}, J=5.1,1.4 \mathrm{~Hz}, 1 \mathrm{H}), 7.15(\mathrm{dd}, J=5.0,3.8 \mathrm{~Hz}, 1 \mathrm{H}), 3.46(\mathrm{~s}, 3 \mathrm{H}), 3.38(\mathrm{~s}, 1 \mathrm{H}) .{ }^{13} \mathrm{C}$ NMR $\left(126 \mathrm{MHz}, \mathrm{CDCl}_{3}\right) \delta 151.82,138.04,135.54,134.64,127.75,82.84,75.31,33.76$. HRMS ESI $[\mathrm{M}+\mathrm{H}]^{+}$calculated for $\mathrm{C}_{8} \mathrm{H}_{8} \mathrm{NO}_{3} \mathrm{~S}_{2} 229.9940$, found 229.9937 .<smiles>C#CC(=O)N(C)S(=O)(=O)c1ccc(Cl)nc1</smiles>

$N$-((6-chloropyridin-3-yl)sulfonyl)- $N$-methylpropiolamide (2o). Following the literature procedure, ${ }^{3}$ the title compound $(274 \mathrm{mg}$ ) was obtained in $53 \%$ yield starting from 6-chloro- $N$ methylpyridine-3-sulfonamide (414 mg, $2.0 \mathrm{mmol}) .{ }^{1} \mathrm{H} \mathrm{NMR}\left(400 \mathrm{MHz}, \mathrm{CDCl}_{3}\right) \delta 8.94$ (dd, $J$ = 2.6, $0.7 \mathrm{~Hz}, 1 \mathrm{H}), 8.28(\mathrm{dd}, J=8.4,2.6 \mathrm{~Hz}, 1 \mathrm{H}), 7.52$ (dd, $J=8.4,0.7 \mathrm{~Hz}, 1 \mathrm{H}), 3.55(\mathrm{~s}, 3 \mathrm{H})$, 3.37 (s, 1H). ${ }^{13} \mathrm{C}$ NMR $\left(126 \mathrm{MHz}, \mathrm{CDCl}_{3}\right) \delta 157.24,151.86,149.67,139.31,133.93,124.71$, 83.01, 74.78, 34.14. HRMS ESI $[\mathrm{M}+\mathrm{H}]^{+}$calculated for $\mathrm{C}_{9} \mathrm{H}_{8} \mathrm{ClN}_{2} \mathrm{O}_{3} \mathrm{~S} 258.9939$, found 258.9932 .<smiles>C#CC(=O)N(CCCC=C)S(=O)(=O)c1ccc(C)cc1</smiles> 
$N$-(pent-4-en-1-yl)- $N$-tosylpropiolamide (2p). Following the literature procedure, ${ }^{3}$ the title compound (326 mg) was obtained in $56 \%$ yield starting from 4-methyl- $N$-(pent-4-en-1yl)benzenesulfonamide (478 mg, $2.0 \mathrm{mmol}) .{ }^{1} \mathrm{H} \mathrm{NMR}\left(400 \mathrm{MHz}, \mathrm{CDCl}_{3}\right) \delta 7.87$ (d, $J=8.2$ $\mathrm{Hz}, 2 \mathrm{H}), 7.32(\mathrm{~d}, J=8.2 \mathrm{~Hz}, 2 \mathrm{H}), 5.92-5.69(\mathrm{~m}, 1 \mathrm{H}), 5.22-4.93(\mathrm{~m}, 2 \mathrm{H}), 4.23-3.81(\mathrm{~m}$, 2H), 3.25 (s, 1H), 2.43 (s, 3H), $2.18-2.11(\mathrm{~m}, 2 \mathrm{H}), 1.90(\mathrm{p}, J=7.7 \mathrm{~Hz}, 2 \mathrm{H}) .{ }^{13} \mathrm{C}$ NMR $(126$ $\left.\mathrm{MHz}, \mathrm{CDCl}_{3}\right) \delta 151.74,145.43,136.97,135.68,129.62,128.76,115.74,81.40,75.32,47.56$, 30.84, 29.39, 21.77. HRMS ESI [M+H] $]^{+}$calculated for $\mathrm{C}_{15} \mathrm{H}_{18} \mathrm{NO}_{3} \mathrm{~S} 292.1002$, found 292.1006.

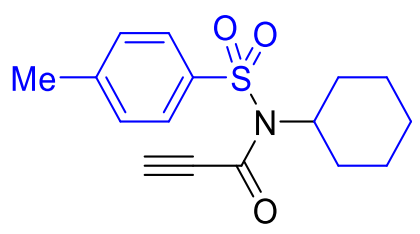

$N$-cyclohexyl- $N$-tosylpropiolamide (2q). Following the literature procedure, ${ }^{3}$ the title compound (305 mg) was obtained in 50\% yield starting from $N$-cyclohexyl-4methylbenzenesulfonamide $(507 \mathrm{mg}, 2.0 \mathrm{mmol})$. The spectra data are in agreement with this literature.<smiles>C#CC(=O)N(CC(C)C)S(=O)(=O)c1ccc(C)cc1</smiles>

$N$-isobutyl- $N$-tosylpropiolamide (2r). Following the literature procedure, ${ }^{3}$ the title compound $(291 \mathrm{mg})$ was obtained in $52 \%$ yield starting from $\mathrm{N}$-isobutyl-4-methylbenzenesulfonamide (454 mg, $2.0 \mathrm{mmol}) .{ }^{1} \mathrm{H} \mathrm{NMR}\left(400 \mathrm{MHz}, \mathrm{CDCl}_{3}\right) \delta 7.88(\mathrm{~d}, J=8.4 \mathrm{~Hz}, 2 \mathrm{H}), 7.32(\mathrm{~d}, J=8.1$ $\mathrm{Hz}, 2 \mathrm{H}), 3.84(\mathrm{~d}, J=7.6 \mathrm{~Hz}, 2 \mathrm{H}), 3.24(\mathrm{~s}, 1 \mathrm{H}), 2.44(\mathrm{~s}, 3 \mathrm{H}), 2.30-2.19(\mathrm{~m}, 1 \mathrm{H}), 0.99(\mathrm{~d}, J=$ $6.7 \mathrm{~Hz}, 6 \mathrm{H}) .{ }^{13} \mathrm{C} \mathrm{NMR}\left(126 \mathrm{MHz}, \mathrm{CDCl}_{3}\right) \delta 152.27,145.38,135.80,129.57$ (2C), $128.99(2 \mathrm{C})$, 81.75, 75.64, 54.91, 29.28, 21.83, 19.95 (2C). HRMS ESI $[\mathrm{M}+\mathrm{H}]^{+}$calculated for $\mathrm{C}_{14} \mathrm{H}_{18} \mathrm{NO}_{3} \mathrm{~S}$ 280.1002 , found 280.0996 .<smiles>C#CC(=O)N(C(Cc1ccccc1)C(C)=O)S(=O)(=O)c1ccc(C)cc1</smiles>

from $D$-phenylalanine

Methyl $N$-propioloyl- $N$-tosyl- $D$-phenylalaninate (S-6d). Following the literature procedure, ${ }^{3}$ the compound (162 mg) was obtained in $42 \%$ yield starting from methyl tosyl- $D$ phenylalaninate $(333 \mathrm{mg}, 1.0 \mathrm{mmol}) .{ }^{1} \mathrm{H} \mathrm{NMR}\left(400 \mathrm{MHz}, \mathrm{CDCl}_{3}\right) \delta 7.60(\mathrm{~d}, J=8.0 \mathrm{~Hz}, 2 \mathrm{H})$, $7.37-7.24(\mathrm{~m}, 5 \mathrm{H}), 7.20(\mathrm{~d}, J=8.1 \mathrm{~Hz}, 2 \mathrm{H}), 5.40(\mathrm{dd}, J=9.6,5.2 \mathrm{~Hz}, 1 \mathrm{H}), 3.79$ (s, 3H), 3.73 $-3.63(\mathrm{~m}, 1 \mathrm{H}), 3.37(\mathrm{dd}, J=14.4,9.6 \mathrm{~Hz}, 1 \mathrm{H}), 3.17(\mathrm{~s}, 1 \mathrm{H}), 2.41(\mathrm{~s}, 3 \mathrm{H}) .{ }^{13} \mathrm{C}$ NMR $(126 \mathrm{MHz}$, $\left.\mathrm{CDCl}_{3}\right) \delta 169.30,151.38,145.41,136.97,135.42,129.92,129.40,129.34,128.93,127.24$, 82.76, 75.16, 61.93, 53.10, 36.35, 21.81. HRMS ESI $[\mathrm{M}+\mathrm{H}]^{+}$calculated for $\mathrm{C}_{20} \mathrm{H}_{20} \mathrm{NO}_{5} \mathrm{~S}$ 386.1057 , found 386.1048 . 
<smiles>C#CC(=O)N(C(C)C(C)=O)S(=O)(=O)c1ccc(C)cc1</smiles>

from $D$-alanine

Methyl $\boldsymbol{N}$-propioloyl- $\boldsymbol{N}$-tosyl- $\boldsymbol{D}$-alaninate $(\mathbf{S}-6 \mathrm{e})$. Following the literature procedure, ${ }^{3}$ the compound (139 mg) was obtained in $45 \%$ yield starting from methyl tosyl- $D$-alaninate (257 $\mathrm{mg}, 1.0 \mathrm{mmol}) .{ }^{1} \mathrm{H} \mathrm{NMR}\left(400 \mathrm{MHz}, \mathrm{CDCl}_{3}\right) \delta 7.94(\mathrm{~d}, J=8.4 \mathrm{~Hz}, 2 \mathrm{H}), 7.35(\mathrm{~d}, J=8.0 \mathrm{~Hz}$, $2 \mathrm{H}), 5.21$ (q, $J=7.0 \mathrm{~Hz}, 1 \mathrm{H}), 3.72(\mathrm{~s}, 3 \mathrm{H}), 3.26(\mathrm{~s}, 1 \mathrm{H}), 2.45(\mathrm{~s}, 3 \mathrm{H}), 1.71$ (d, $J=6.8 \mathrm{~Hz}, 3 \mathrm{H})$. ${ }^{13} \mathrm{C} \mathrm{NMR}\left(126 \mathrm{MHz}, \mathrm{CDCl}_{3}\right) \delta 169.80,150.93,145.68,135.63,129.64,129.05,82.74,75.18$, 55.90, 52.93, 21.86, 16.74. HRMS ESI $[\mathrm{M}+\mathrm{H}]^{+}$calculated for $\mathrm{C}_{14} \mathrm{H}_{16} \mathrm{NO}_{5} \mathrm{~S} 310.0744$, found 310.0736 .<smiles>C#CC(=O)N(C[C@]1(C)CC[C@H]2c3ccc(C(C)C)cc3CC[C@H]2C1)S(=O)(=O)c1ccc(C)cc1</smiles>

from (+)-dehydroabietylamine

$N$-(((2R,4aS,10aS)-7-isopropyl-2,4a-dimethyl-1,2,3,4,4a,9,10,10a-

octahydrophenanthren-2-yl)methyl)- $N$-tosylpropiolamide (S-6f). Following the literature procedure, ${ }^{3}$ the compound $(206 \mathrm{mg}$ ) was obtained in $42 \%$ yield starting from $\mathrm{N}$ $(((2 R, 4 \mathrm{a} S, 10 \mathrm{a} S)$-7-isopropyl-2,4a-dimethyl-1,2,3,4,4a,9,10,10a-octahydrophenanthren-2yl)methyl)-4-methylbenzenesulfonamide (440 mg, $1.0 \mathrm{mmol}) .{ }^{1} \mathrm{H} \mathrm{NMR}\left(500 \mathrm{MHz}, \mathrm{CDCl}_{3}\right) \delta$ $7.80(\mathrm{~d}, J=8.2 \mathrm{~Hz}, 2 \mathrm{H}), 7.28(\mathrm{~d}, J=7.9 \mathrm{~Hz}, 2 \mathrm{H}), 7.17(\mathrm{~d}, J=8.2 \mathrm{~Hz}, 1 \mathrm{H}), 7.00$ (dd, $J=8.2$, $2.0 \mathrm{~Hz}, 1 \mathrm{H}), 6.91(\mathrm{~d}, J=1.9 \mathrm{~Hz}, 1 \mathrm{H}), 4.25(\mathrm{~d}, J=14.8 \mathrm{~Hz}, 1 \mathrm{H}), 3.95(\mathrm{~d}, J=14.8 \mathrm{~Hz}, 1 \mathrm{H}), 3.25$ (s, 1H), 2.96 (dd, $J=8.3,3.4 \mathrm{~Hz}, 2 \mathrm{H}), 2.84$ (hept, $J=6.8 \mathrm{~Hz}, 1 \mathrm{H}), 2.42(\mathrm{~s}, 3 \mathrm{H}), 2.34-2.26$ $(\mathrm{m}, 1 \mathrm{H}), 2.02-1.95(\mathrm{~m}, 1 \mathrm{H}), 1.90-1.80(\mathrm{~m}, 1 \mathrm{H}), 1.76-1.64(\mathrm{~m}, 3 \mathrm{H}), 1.56-1.36(\mathrm{~m}, 3 \mathrm{H})$, $1.25(\mathrm{~s}, 3 \mathrm{H}), 1.23(\mathrm{~d}, J=6.9 \mathrm{~Hz}, 6 \mathrm{H}), 1.11(\mathrm{~s}, 3 \mathrm{H}) .{ }^{13} \mathrm{C} \mathrm{NMR}\left(126 \mathrm{MHz}, \mathrm{CDCl}_{3}\right) \delta 153.17$, 147.43, 145.82, 145.21, 136.08, 134.62, 129.51, 128.93, 126.97, 124.08, 124.00, 82.80, 76.13, 58.60, 39.28, 38.26, 37.91, 36.93, 33.60, 30.07, 29.85, 25.81, 24.14, 24.13, 21.80, 19.68, 18.60, 18.52. HRMS ESI $[\mathrm{M}+\mathrm{H}]^{+}$calculated for $\mathrm{C}_{30} \mathrm{H}_{38} \mathrm{NO}_{3} \mathrm{~S} 492.2567$, found 492.2560 . 


\section{Synthesis of isothiazolidin-3-one-1,1-dioxides via neutral eosin Y}

\section{photocatalysis}

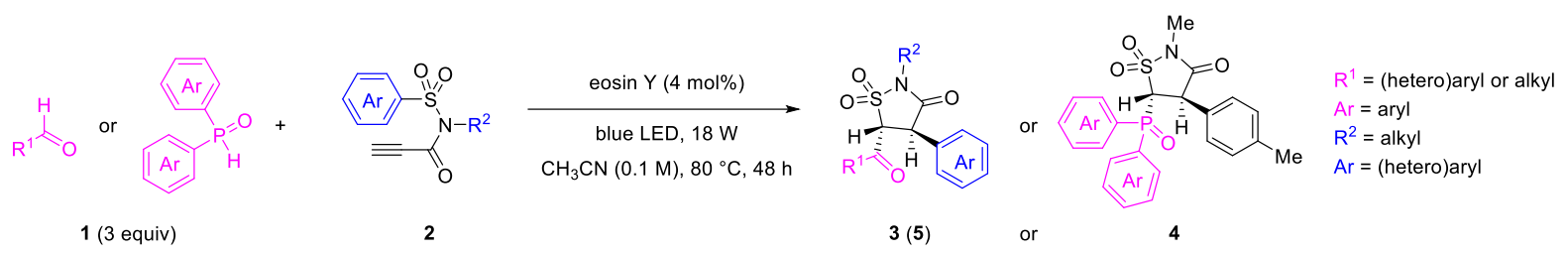

General procedure for the synthesis of isothiazolidin-3-one-1,1-dioxides

A $20 \mathrm{~mL}$ Schlenk tube equipped with a magnetic stir bar was charged with eosin Y $(0.004$ mmol, $2.6 \mathrm{mg})$, aldehyde or $H$-phosphine oxide $1(0.3 \mathrm{mmol})$, and $N$-arylsulfonyl propiolamide $2(0.1 \mathrm{mmol})$. Then, anhydrous acetonitrile $(1.0 \mathrm{~mL})$ was added. The Schlenk tube was connected to Schlenk line and freeze-pump-thaw was performed for three times to completely remove air inside the reaction mixture. Eventually the Schlenk tube was refilled with an atmosphere of argon at room temperature and sealed. The reaction was placed in a water bath covered by top oil layer under a blue LED strip ( 2 meters, $18 \mathrm{~W}$ ). The reaction was running at $80{ }^{\circ} \mathrm{C}$ (the reaction with $\mathrm{H}$-phosphine oxide was performed at room temperature using fan to cool down the reaction mixture) and stopped after $48 \mathrm{~h}$. The solvent was removed under reduced pressure and the crude mixture was purified by silica gel column chromatography or prepared TLC (eluent: hexane/diethyl ether or hexane/ethyl acetate; 10/1 - 3/1) to give the corresponding product. The diastereoselectivity for the product 3a was confirmed by X-ray crystallographic analysis and assumed for other analogs.

\section{Gram-scale synthesis of isothiazolidin-3-one-1,1-dioxides}

A $100 \mathrm{~mL}$ Schlenk tube equipped with a magnetic stir bar was charged with eosin Y (0.2 mmol, $130 \mathrm{mg}$ ), 4-fluoro-benzaldehyde $\mathbf{1 i}$ (15 mmol, $1.862 \mathrm{~g}$ ), and $N$-methyl- $N$-tosyl propiolamide 2a $(5 \mathrm{mmol}, 1.186 \mathrm{~g})$. Then, anhydrous acetonitrile $(50.0 \mathrm{~mL})$ was added. The Schlenk tube was connected to Schlenk line and freeze-pump-thaw was performed for three times to completely remove air inside the reaction mixture. Eventually the Schlenk tube was refilled with an atmosphere of argon at room temperature and sealed. The reaction was placed in a water bath covered by top oil layer under a blue LED strip ( 2 meters, $18 \mathrm{~W}$ ). The reaction was heated at $80{ }^{\circ} \mathrm{C}$ and stopped after $4 \mathrm{~d}$, then solvent was removed under reduced pressure. The crude product was purified by silica gel column chromatography (eluent: hexane/diethyl ether $=5 / 1)$ to give the corresponding product $3 \mathbf{i}(1.5 \mathrm{~g})$ in $83 \%$ yield.<smiles>Cc1ccc([C@H]2C(=O)N(C)S(=O)(=O)[C@H]2C(=O)c2ccccc2)cc1</smiles> 
( \pm -5-Benzoyl-2-methyl-4-(p-tolyl)isothiazolidin-3-one 1,1-dioxide (3a). Following the general procedure, the tittle compound $\left(29.9 \mathrm{mg}\right.$ ) was obtained in $87 \%$ yield. ${ }^{1} \mathrm{H}$ NMR (400 $\left.\mathrm{MHz}, \mathrm{CDCl}_{3}\right) \delta 8.11(\mathrm{dd}, J=8.5,1.3 \mathrm{~Hz}, 2 \mathrm{H}), 7.73-7.65(\mathrm{~m}, 1 \mathrm{H}), 7.60-7.50(\mathrm{~m}, 2 \mathrm{H}), 7.22$ $(\mathrm{d}, J=8.3 \mathrm{~Hz}, 2 \mathrm{H}), 7.17(\mathrm{~d}, J=8.0 \mathrm{~Hz}, 2 \mathrm{H}), 5.46(\mathrm{~d}, J=7.8 \mathrm{~Hz}, 1 \mathrm{H}), 5.19(\mathrm{~d}, J=7.7 \mathrm{~Hz}, 1 \mathrm{H})$, $3.20(\mathrm{~s}, 3 \mathrm{H}), 2.32(\mathrm{~s}, 3 \mathrm{H}) .{ }^{13} \mathrm{C} \mathrm{NMR}\left(126 \mathrm{MHz}, \mathrm{CDCl}_{3}\right) \delta 185.02,166.82,138.94,135.40$, 135.36, 131.20, 130.24, 129.57, 129.40, 128.59, 70.00, 50.20, 25.09, 21.25. HRMS ESI [M$\mathrm{H}]^{-}$calculated for $\mathrm{C}_{18} \mathrm{H}_{16} \mathrm{NO}_{4} \mathrm{~S} 342.0806$, found 342.0809. Recrystallization from diethyl ether afforded single crystals of 3a suitable for X-ray diffraction analysis, which unambiguously confirmed the diastereochemistry (Figure S2).

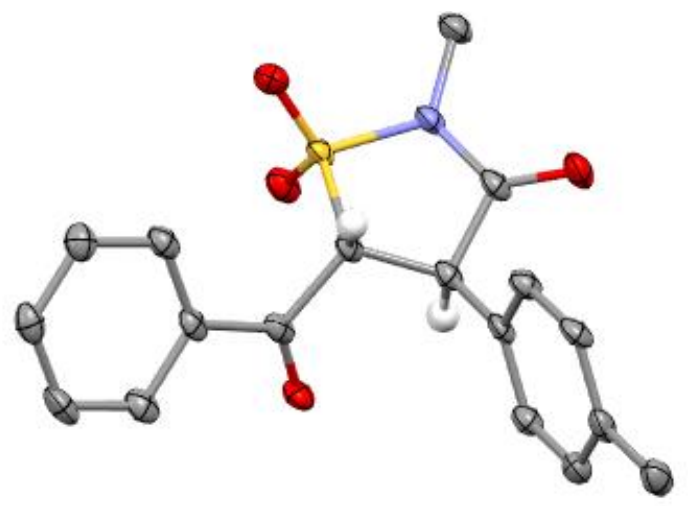

Figure S2. ORTEP diagram of 3a. Thermal ellipsoids drawn at 50\% probability.<smiles>COc1ccc(C(=O)[C@H]2[C@H](c3ccc(C)cc3)C(=O)N(C)S2(=O)=O)cc1</smiles>

( \pm )-(4R,5R)-5-(4-Methoxybenzoyl)-2-methyl-4-(p-tolyl)isothiazolidin-3-one 1,1-dioxide (3b). Following the general procedure, the tittle compound $\mathbf{3 b}(25.7 \mathrm{mg})$ was obtained in $69 \%$ yield. ${ }^{1} \mathrm{H}$ NMR $\left(400 \mathrm{MHz}, \mathrm{CDCl}_{3}\right) \delta 8.08(\mathrm{~d}, J=9.0 \mathrm{~Hz}, 2 \mathrm{H}), 7.21(\mathrm{~d}, J=8.3 \mathrm{~Hz}, 2 \mathrm{H}), 7.17$ $(\mathrm{d}, J=8.1 \mathrm{~Hz}, 2 \mathrm{H}), 6.99$ (d, $J=9.0 \mathrm{~Hz}, 2 \mathrm{H}), 5.39$ (d, $J=7.9 \mathrm{~Hz}, 1 \mathrm{H}), 5.19$ (d, $J=7.9 \mathrm{~Hz}, 1 \mathrm{H})$, 3.89 (s, 3H), 3.19 (s, 3H), 2.32 (s, 3H). ${ }^{13} \mathrm{C} \mathrm{NMR}\left(126 \mathrm{MHz}, \mathrm{CDCl}_{3}\right) \delta 182.85,167.03,165.44$, 138.83, 132.11, 131.29, 130.19, 128.63, 128.48, 114.67, 69.76, 55.86, 50.10, 25.05, 21.25. HRMS ESI [M-H] $]^{-}$calculated for $\mathrm{C}_{19} \mathrm{H}_{18} \mathrm{NO}_{5} \mathrm{~S} 372.0911$, found 372.0914.<smiles>CC(=O)Oc1ccc(C(=O)[C@H]2[C@H](c3ccc(C)cc3)C(=O)N(C)S2(=O)=O)cc1</smiles>

\section{4-(( \pm$)-(4 R, 5 R)-2-M e t h y l-1,1-d i o x i d o-3-0 x o-4-(p$-tolyl)isothiazolidine-5-carbonyl)phenyl}

acetate (3c). Following the general procedure, the tittle compound $(30.9 \mathrm{mg})$ was obtained in $77 \%$ yield. ${ }^{1} \mathrm{H}$ NMR $\left(500 \mathrm{MHz}, \mathrm{CDCl}_{3}\right) \delta 8.15(\mathrm{~d}, J=8.8 \mathrm{~Hz}, 2 \mathrm{H}), 7.29(\mathrm{~d}, J=8.8 \mathrm{~Hz}, 2 \mathrm{H})$, $7.19(\mathrm{~d}, J=8.5 \mathrm{~Hz}, 2 \mathrm{H}), 7.17(\mathrm{~d}, J=8.2 \mathrm{~Hz}, 2 \mathrm{H}), 5.40(\mathrm{~d}, J=7.9 \mathrm{~Hz}, 1 \mathrm{H}), 5.17(\mathrm{~d}, J=7.9 \mathrm{~Hz}$, $1 \mathrm{H}), 3.20$ (s, 3H), 2.33 (s, 3H), 2.32 (s, 3H). $\left.{ }^{13} \mathrm{C} \mathrm{NMR} \mathrm{(126} \mathrm{MHz,} \mathrm{CDCl}_{3}\right) \delta 183.75,168.56$, 
$166.80,156.19,139.01,132.80,131.32,131.00,130.27,128.56,122.67,70.06,50.22,25.12$, 21.30, 21.26. HRMS ESI [M-H] $]^{-}$calculated for $\mathrm{C}_{20} \mathrm{H}_{18} \mathrm{NO}_{6} \mathrm{~S} 400.0860$, found 400.0852.<smiles></smiles>

( \pm )-(4R,5R)-5-(4-Hydroxybenzoyl)-2-methyl-4-(p-tolyl)isothiazolidin-3-one 1,1-dioxide (3d). Following the general procedure, the title compound $(23.3 \mathrm{mg})$ was obtained in $65 \%$ yield. ${ }^{1} \mathrm{H}$ NMR $\left(500 \mathrm{MHz}, \mathrm{CDCl}_{3}\right) \delta 8.00(\mathrm{~d}, J=8.9 \mathrm{~Hz}, 2 \mathrm{H}), 7.20(\mathrm{~d}, J=8.3 \mathrm{~Hz}, 2 \mathrm{H}), 7.16(\mathrm{~d}, J=$ $8.0 \mathrm{~Hz}, 2 \mathrm{H}), 6.89$ (d, $J=8.8 \mathrm{~Hz}, 2 \mathrm{H}), 5.38(\mathrm{~d}, J=7.9 \mathrm{~Hz}, 1 \mathrm{H}), 5.19$ (d, $J=7.8 \mathrm{~Hz}, 1 \mathrm{H}), 3.19$ $(\mathrm{s}, 3 \mathrm{H}), 2.31(\mathrm{~s}, 3 \mathrm{H}) .{ }^{13} \mathrm{C} \mathrm{NMR}\left(126 \mathrm{MHz}, \mathrm{CDCl}_{3}\right) \delta 182.58,167.01,162.41,138.76,132.25$, $131.07,130.08,128.48,128.22,116.17,69.57,50.01,24.96,21.11$. HRMS ESI [M-H] ${ }^{-}$ calculated for $\mathrm{C}_{18} \mathrm{H}_{16} \mathrm{NO}_{5} \mathrm{~S} 358.0755$, found 358.0759.<smiles>CCCCCNc1ccc(C(=O)[C@H]2[C@@H](c3ccc(C)cc3)C(=O)N(C)S2(=O)=O)cc1</smiles>

$N$-(4-( \pm )-(4R,5R)-(2-methyl-1,1-dioxido-3-oxo-4-(p-tolyl)isothiazolidine-5-

carbonyl)phenyl)acetamide (3e). Following the general procedure, the title compound (26.1 $\mathrm{mg}$ ) was obtained in $65 \%$ yield. ${ }^{1} \mathrm{H} \mathrm{NMR}\left(500 \mathrm{MHz}, \mathrm{CDCl}_{3}\right) \delta 8.06(\mathrm{~d}, J=8.9 \mathrm{~Hz}, 2 \mathrm{H}), 7.66$ $(\mathrm{d}, J=8.8 \mathrm{~Hz}, 2 \mathrm{H}), 7.49$ (s, 1H), $7.21(\mathrm{~d}, J=8.3 \mathrm{~Hz}, 2 \mathrm{H}), 7.17(\mathrm{~d}, J=8.1 \mathrm{~Hz}, 2 \mathrm{H}), 5.40(\mathrm{~d}, J$ $=7.8 \mathrm{~Hz}, 1 \mathrm{H}), 5.18(\mathrm{~d}, J=7.9 \mathrm{~Hz}, 1 \mathrm{H}), 3.19(\mathrm{~s}, 3 \mathrm{H}), 2.31(\mathrm{~s}, 3 \mathrm{H}), 2.20(\mathrm{~s}, 3 \mathrm{H}) .{ }^{13} \mathrm{C} \mathrm{NMR}(126$ $\left.\mathrm{MHz}, \mathrm{CDCl}_{3}\right) \delta 183.21,168.66,166.95,144.36,138.94,131.21,131.18,130.85,130.24$, 128.61, 119.25, 69.83, 50.15, 25.09, 24.97, 21.25. HRMS ESI [M-H] ${ }^{-}$calculated for $\mathrm{C}_{20} \mathrm{H}_{19} \mathrm{~N}_{2} \mathrm{O}_{5} \mathrm{~S} 399.1020$, found 399.1016.<smiles>Cc1ccc(C(=O)[C@H]2[C@H](c3ccc(C)cc3)C(=O)N(C)S2(=O)=O)cc1</smiles>

$( \pm)$-(4R,5R)-2-Methyl-5-(4-methylbenzoyl)-4-( $p$-tolyl)isothiazolidin-3-one 1,1-dioxide (3f). Following the general procedure, the title compound $(22.9 \mathrm{mg})$ was obtained in $64 \%$ yield. ${ }^{1} \mathrm{H}$ $\operatorname{NMR}\left(500 \mathrm{MHz}, \mathrm{CDCl}_{3}\right) \delta 8.00(\mathrm{~d}, J=8.3 \mathrm{~Hz}, 2 \mathrm{H}), 7.34(\mathrm{~d}, J=8.0 \mathrm{~Hz}, 2 \mathrm{H}), 7.21(\mathrm{~d}, J=8.3$ $\mathrm{Hz}, 2 \mathrm{H}), 7.17$ (d, $J=8.0 \mathrm{~Hz}, 2 \mathrm{H}), 5.43$ (d, $J=7.8 \mathrm{~Hz}, 1 \mathrm{H}), 5.18$ (d, $J=7.7 \mathrm{~Hz}, 1 \mathrm{H}), 3.19$ (s, $3 \mathrm{H}), 2.43(\mathrm{~s}, 3 \mathrm{H}), 2.32$ (s, 3H). ${ }^{13} \mathrm{C} \mathrm{NMR}\left(126 \mathrm{MHz}, \mathrm{CDCl}_{3}\right) \delta 184.22,166.79,146.75,138.74$, 132.84, 131.12, 130.08, 129.97, 129.56, 128.47, 69.75, 49.98, 24.94, 21.88, 21.12. HRMS ESI $[\mathrm{M}-\mathrm{H}]^{-}$calculated for $\mathrm{C}_{19} \mathrm{H}_{18} \mathrm{NO}_{4} \mathrm{~S} 356.0962$, found 356.0957 . 
<smiles>Cc1ccc([C@H]2C(=O)N(C)S(=O)(=O)[C@H]2C(=O)c2ccc(Cl)cc2)cc1</smiles>

( \pm )-(4R,5R)-5-(4-Chlorobenzoyl)-2-methyl-4-(p-tolyl)isothiazolidin-3-one 1,1-dioxide (3g). Following the general procedure, the title compound $(21.9 \mathrm{mg})$ was obtained in $58 \%$ yield. ${ }^{1} \mathrm{H}$ $\operatorname{NMR}\left(400 \mathrm{MHz}, \mathrm{CDCl}_{3}\right) \delta 8.05(\mathrm{~d}, J=8.8 \mathrm{~Hz}, 2 \mathrm{H}), 7.52(\mathrm{~d}, J=8.8 \mathrm{~Hz}, 2 \mathrm{H}), 7.21(\mathrm{~d}, J=8.4$ $\mathrm{Hz}, 2 \mathrm{H}), 7.18(\mathrm{~d}, J=8.4 \mathrm{~Hz}, 2 \mathrm{H}), 5.38(\mathrm{~d}, J=8.0 \mathrm{~Hz}, 1 \mathrm{H}), 5.16(\mathrm{~d}, J=7.8 \mathrm{~Hz}, 1 \mathrm{H}), 3.19$ (s, $3 \mathrm{H}), 2.32(\mathrm{~s}, 3 \mathrm{H}) .{ }^{13} \mathrm{C} \mathrm{NMR}\left(126 \mathrm{MHz}, \mathrm{CDCl}_{3}\right) \delta 183.79,166.54,142.19,138.91,133.51$, $130.87,130.74,130.15,129.67,128.45,69.94,49.95,25.00,21.13$. HRMS ESI [M-H] ${ }^{-}$ calculated for $\mathrm{C}_{18} \mathrm{H}_{15} \mathrm{ClNO}_{4} \mathrm{~S} 376.0416$, found 376.0409.<smiles>Cc1ccc([C@H]2C(=O)N(C)S(=O)(=O)[C@H]2C(=O)c2ccc(Br)cc2)cc1</smiles>

( \pm )-(4R,5R)-5-(4-bromobenzoyl)-2-methyl-4-(p-tolyl)isothiazolidin-3-one 1,1-dioxide (3h). Following the general procedure, the title compound $(25.3 \mathrm{mg})$ was obtained in $60 \%$ yield. ${ }^{1} \mathrm{H}$ NMR (400 MHz, $\left.\mathrm{CDCl}_{3}\right) \delta 7.96(\mathrm{~d}, J=8.7 \mathrm{~Hz}, 2 \mathrm{H}), 7.69(\mathrm{~d}, J=8.7 \mathrm{~Hz}, 2 \mathrm{H}), 7.21(\mathrm{~d}, J=8.5$ $\mathrm{Hz}, 2 \mathrm{H}), 7.18$ (d, $J=8.3 \mathrm{~Hz}, 2 \mathrm{H}), 5.38$ (d, $J=7.8 \mathrm{~Hz}, 1 \mathrm{H}), 5.16$ (d, $J=7.7 \mathrm{~Hz}, 1 \mathrm{H}), 3.19$ (s, $3 \mathrm{H}), 2.32(\mathrm{~s}, 3 \mathrm{H}) .{ }^{13} \mathrm{C} \mathrm{NMR}\left(126 \mathrm{MHz}, \mathrm{CDCl}_{3}\right) \delta 184.18,166.66,139.05,134.03,132.80$, $131.23,130.99,130.86,130.28,128.58,70.05,50.07,25.13,21.26$. HRMS ESI [M-H] ${ }^{-}$ calculated for $\mathrm{C}_{18} \mathrm{H}_{15} \mathrm{BrNO}_{4} \mathrm{~S} 419.9911$, found 419.9916 .<smiles>Cc1ccc([C@H]2C(=O)N(C)S(=O)(=O)[C@H]2C(=O)c2ccc(F)cc2)cc1</smiles>

$( \pm)$-(4R,5R)-5-(4-Fluorobenzoyl)-2-methyl-4-(p-tolyl)isothiazolidin-3-one 1,1-dioxide (3i). Following the general procedure, the title compound $(34.3 \mathrm{mg})$ was obtained in $95 \%$ yield. ${ }^{1} \mathrm{H}$ $\operatorname{NMR}\left(400 \mathrm{MHz}, \mathrm{CDCl}_{3}\right) \delta 8.15(\mathrm{dd}, J=9.0,5.2 \mathrm{~Hz}, 2 \mathrm{H}), 7.25-7.15(\mathrm{~m}, 6 \mathrm{H}), 5.39(\mathrm{~d}, J=7.9$ $\mathrm{Hz}, 1 \mathrm{H}), 5.17$ (d, $J=7.9 \mathrm{~Hz}, 1 \mathrm{H}), 3.19$ (s, 3H), 2.32 (s, 3H). ${ }^{13} \mathrm{C} \mathrm{NMR}\left(126 \mathrm{MHz}, \mathrm{CDCl}_{3}\right) \delta$ $183.39,167.16\left(\mathrm{~d},{ }^{1} J_{\mathrm{C}-\mathrm{F}}=259.9 \mathrm{~Hz}\right), 166.73,139.03,132.47\left(\mathrm{~d},{ }^{3} J_{\mathrm{C}-\mathrm{F}}=10.1 \mathrm{~Hz}\right), 131.85(\mathrm{~d}$, $\left.{ }^{4} J_{\mathrm{C}-\mathrm{F}}=2.9 \mathrm{~Hz}\right), 131.05,130.27,128.60,116.77\left(\mathrm{~d},{ }^{2} J_{\mathrm{C}-\mathrm{F}}=22.3 \mathrm{~Hz}\right), 70.07,50.12,25.12,21.26$. ${ }^{19} \mathrm{~F}$ NMR $\left(377 \mathrm{MHz}, \mathrm{CDCl}_{3}\right) \delta-100.49$. HRMS ESI $[\mathrm{M}-\mathrm{H}]^{-}$calculated for $\mathrm{C}_{18} \mathrm{H}_{15} \mathrm{FNO}_{4} \mathrm{~S}$ 360.0711 , found 360.0705 . 


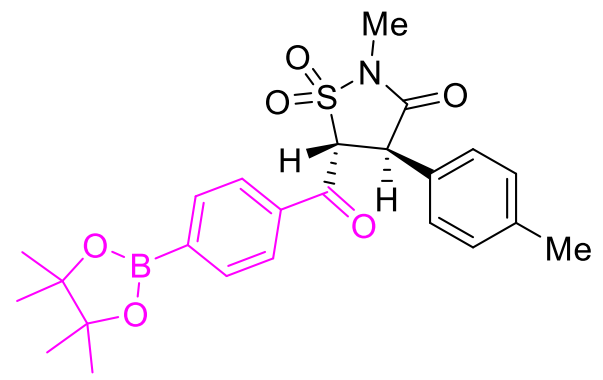

( \pm )-(4R,5R)-2-Methyl-5-(4-(4,4,5,5-tetramethyl-1,3,2-dioxaborolan-2-yl)benzoyl)-4-( $p$ tolyl)isothiazolidin-3-one 1,1-dioxide (3j). Following the general procedure, the title compound $(26 \mathrm{mg})$ was obtained in $56 \%$ yield. ${ }^{1} \mathrm{H} \mathrm{NMR}\left(400 \mathrm{MHz}, \mathrm{CDCl}_{3}\right) \delta 8.06(\mathrm{~d}, J=8.4$ $\mathrm{Hz}, 2 \mathrm{H}), 7.96(\mathrm{~d}, J=8.4 \mathrm{~Hz}, 2 \mathrm{H}), 7.22(\mathrm{~d}, J=8.3 \mathrm{~Hz}, 2 \mathrm{H}), 7.17(\mathrm{~d}, J=8.1 \mathrm{~Hz}, 2 \mathrm{H}), 5.46(\mathrm{~d}, J$ $=7.7 \mathrm{~Hz}, 1 \mathrm{H}), 5.17(\mathrm{~d}, J=7.6 \mathrm{~Hz}, 1 \mathrm{H}), 3.18(\mathrm{~s}, 3 \mathrm{H}), 2.32(\mathrm{~s}, 3 \mathrm{H}), 1.35(\mathrm{~s}, 12 \mathrm{H}) .{ }^{13} \mathrm{C} \mathrm{NMR}(126$ $\left.\mathrm{MHz}, \mathrm{CDCl}_{3}\right) \delta 185.39,166.83,138.95,137.03,135.56,131.17,130.25,128.61,128.41,84.60$, $70.03,50.15,25.09,25.04,24.97,21.26$. A signal for the carbon directly attached to the boron atom was not observed. ${ }^{11} \mathrm{~B} \mathrm{NMR}\left(128 \mathrm{MHz}, \mathrm{CDCl}_{3}\right) \delta 31.41$. HRMS ESI [M-H] ${ }^{-}$calculated for $\mathrm{C}_{24} \mathrm{H}_{27} \mathrm{BNO}_{6} \mathrm{~S} 468.1658$, found 468.1644 .

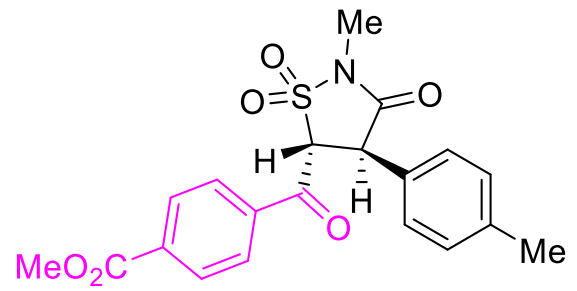

Methyl

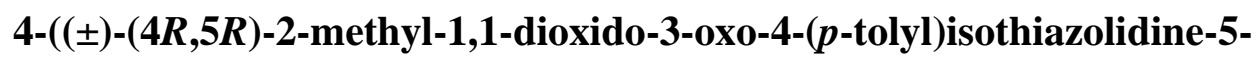
carbonyl)benzoate (3k). Following the general procedure, the title compound (30.5 $\mathrm{mg}$ ) was obtained in $76 \%$ yield. ${ }^{1} \mathrm{H}$ NMR $\left(400 \mathrm{MHz}, \mathrm{CDCl}_{3}\right) \delta 8.19(\mathrm{~d}, J=8.9 \mathrm{~Hz}, 2 \mathrm{H}), 8.16(\mathrm{~d}, J=9.0$ $\mathrm{Hz}, 2 \mathrm{H}), 7.22(\mathrm{~d}, J=8.3 \mathrm{~Hz}, 2 \mathrm{H}), 7.18(\mathrm{~d}, J=8.2 \mathrm{~Hz}, 2 \mathrm{H}), 5.45(\mathrm{~d}, J=7.7 \mathrm{~Hz}, 1 \mathrm{H}), 5.17(\mathrm{~d}, J$ $=7.7 \mathrm{~Hz}, 1 \mathrm{H}), 3.96(\mathrm{~s}, 3 \mathrm{H}), 3.19(\mathrm{~s}, 3 \mathrm{H}), 2.32(\mathrm{~s}, 3 \mathrm{H}) .{ }^{13} \mathrm{C} \mathrm{NMR}\left(126 \mathrm{MHz}, \mathrm{CDCl}_{3}\right) \delta 184.75$, 166.46, 165.65, 138.95, 138.09, 135.70, 130.85, 130.31, 130.16, 129.33, 128.45, 70.17, 52.69, 49.99, 25.01, 21.13. HRMS ESI [M-H] $]^{-}$calculated for $\mathrm{C}_{20} \mathrm{H}_{18} \mathrm{NO}_{6} \mathrm{~S} 400.0860$, found 400.0857 .<smiles>Cc1ccc([C@H]2C(=O)N(C)[C@H](S(=O)[O-])[C@@H]2C(=O)c2ccc(C(F)(F)F)cc2)cc1</smiles>

( \pm )-(4R,5R)-2-methyl-4-( $p$-tolyl)-5-(4-(trifluoromethyl)benzoyl)isothiazolidin-3-one 1,1dioxide (3l). Following the general procedure, the title compound $(32.5 \mathrm{mg})$ was obtained in $79 \%$ yield. ${ }^{1} \mathrm{H} \mathrm{NMR}\left(400 \mathrm{MHz}, \mathrm{CDCl}_{3}\right) \delta 8.23(\mathrm{~d}, J=8.2 \mathrm{~Hz}, 2 \mathrm{H}), 7.81(\mathrm{~d}, J=8.3 \mathrm{~Hz}, 2 \mathrm{H})$, $7.22(\mathrm{~d}, J=8.5 \mathrm{~Hz}, 2 \mathrm{H}), 7.18(\mathrm{~d}, J=8.2 \mathrm{~Hz}, 2 \mathrm{H}), 5.44(\mathrm{~d}, J=7.8 \mathrm{~Hz}, 1 \mathrm{H}), 5.17(\mathrm{~d}, J=7.8 \mathrm{~Hz}$, $1 \mathrm{H}), 3.20(\mathrm{~s}, 3 \mathrm{H}), 2.32$ (s, 3H). ${ }^{13} \mathrm{C} \mathrm{NMR}\left(126 \mathrm{MHz}, \mathrm{CDCl}_{3}\right) \delta 184.55,166.51,139.16,137.78$, $136.41\left(\mathrm{q},{ }^{2} J_{\mathrm{C}-\mathrm{F}}=33.1 \mathrm{~Hz}\right), 130.87,130.32,129.90,128.57,126.45\left(\mathrm{q},{ }^{3} J_{\mathrm{C}-\mathrm{F}}=3.7 \mathrm{~Hz}\right), 125.49$ $\left(\mathrm{q},{ }^{1} J_{\mathrm{C}-\mathrm{F}}=273.5 \mathrm{~Hz}\right), 70.34,50.09,25.16,21.25 .{ }^{19} \mathrm{~F} \mathrm{NMR}\left(377 \mathrm{MHz}, \mathrm{CDCl}_{3}\right) \delta-63.45 . \mathrm{HRMS}$ ESI $[\mathrm{M}-\mathrm{H}]^{-}$calculated for $\mathrm{C}_{19} \mathrm{H}_{15} \mathrm{~F}_{3} \mathrm{NO}_{4} \mathrm{~S} 410.0679$, found 410.0671 . 
<smiles>Cc1ccc([C@H]2C(=O)N(C)S(=O)(=O)[C@H]2C(=O)c2ccc(C#N)cc2)cc1</smiles>

( \pm -4-((4R,5R)-2-methyl-1,1-dioxido-3-oxo-4-(p-tolyl)isothiazolidine-5-

carbonyl)benzonitrile (3m). Following the general procedure, the title compound $(19.5 \mathrm{mg})$ was obtained in 53\% yield. ${ }^{1} \mathrm{H}$ NMR $\left(400 \mathrm{MHz}, \mathrm{CDCl}_{3}\right) \delta 8.21(\mathrm{~d}, J=8.8 \mathrm{~Hz}, 2 \mathrm{H}), 7.84(\mathrm{~d}, J$ $=8.8 \mathrm{~Hz}, 2 \mathrm{H}), 7.21(\mathrm{~d}, J=8.8 \mathrm{~Hz}, 2 \mathrm{H}), 7.18(\mathrm{~d}, J=8.8 \mathrm{~Hz}, 2 \mathrm{H}), 5.40(\mathrm{~d}, J=7.7 \mathrm{~Hz}, 1 \mathrm{H}), 5.15$ $(\mathrm{d}, J=7.7 \mathrm{~Hz}, 1 \mathrm{H}), 3.20$ (s, 3H), 2.32 (s, 3H). ${ }^{13} \mathrm{C} \mathrm{NMR}\left(126 \mathrm{MHz}, \mathrm{CDCl}_{3}\right) \delta 184.36,166.37$, 139.24, 137.97, 133.11, 130.77, 130.36, 129.86, 128.57, 118.54, 117.44, 70.39, 50.06, 25.21, 21.27. HRMS ESI [M-H] $]^{-}$calculated for $\mathrm{C}_{19} \mathrm{H}_{15} \mathrm{~N}_{2} \mathrm{O}_{4} \mathrm{~S} 367.0758$, found 367.0750 .<smiles>Cc1ccc([C@H]2C(=O)N(C)S(=O)(=O)[C@H]2C(=O)c2cccc(C#N)c2)cc1</smiles>

( \pm )-(4R,5R)-3-(2-Methyl-1,1-dioxido-3-oxo-4-(p-tolyl)isothiazolidine-5-

carbonyl)benzonitrile (3n). Following the general procedure, the title compound (17.3 $\mathrm{mg})$ was obtained in $47 \%$ yield. ${ }^{1} \mathrm{H}$ NMR $\left(500 \mathrm{MHz}, \mathrm{CDCl}_{3}\right) \delta 8.38(\mathrm{~s}, 1 \mathrm{H}), 8.33(\mathrm{~d}, J=6.8 \mathrm{~Hz}$, $1 \mathrm{H}), 7.95(\mathrm{~d}, J=6.4 \mathrm{~Hz}, 1 \mathrm{H}), 7.71(\mathrm{t}, J=7.9 \mathrm{~Hz}, 1 \mathrm{H}), 7.25-7.15(\mathrm{~m}, 4 \mathrm{H}), 5.38(\mathrm{~d}, J=7.8 \mathrm{~Hz}$, $1 \mathrm{H}), 5.16(\mathrm{~d}, J=7.8 \mathrm{~Hz}, 1 \mathrm{H}), 3.20(\mathrm{~s}, 3 \mathrm{H}), 2.33(\mathrm{~s}, 3 \mathrm{H}) .{ }^{13} \mathrm{C} \mathrm{NMR}\left(126 \mathrm{MHz}, \mathrm{CDCl}_{3}\right) \delta 183.78$, 166.38, 139.26, 137.94, 136.07, 133.20, 133.04, 130.74, 130.46, 130.38, 128.58, 117.33, 114.30, 70.31, 50.07, 25.22, 21.28. HRMS ESI [M-H] $]^{-}$calculated for $\mathrm{C}_{19} \mathrm{H}_{15} \mathrm{~N}_{2} \mathrm{O}_{4} \mathrm{~S} 367.0758$, found 367.0746 .<smiles>Cc1ccc([C@H]2C(=O)N(C)S(=O)(=O)[C@H]2C(=O)c2cccc(F)c2)cc1</smiles>

( \pm )-(4R,5R)-5-(3-fluorobenzoyl)-2-methyl-4-(p-tolyl)isothiazolidin-3-one 1,1-dioxide (3o). Following the general procedure, the title compound $(24.3 \mathrm{mg})$ was obtained in $68 \%$ yield. ${ }^{1} \mathrm{H}$ $\operatorname{NMR}\left(400 \mathrm{MHz}, \mathrm{CDCl}_{3}\right) \delta 7.91(\mathrm{ddd}, J=7.8,1.7,1.0 \mathrm{~Hz}, 1 \mathrm{H}), 7.79(\mathrm{ddd}, J=9.0,2.5,1.6 \mathrm{~Hz}$, $1 \mathrm{H}), 7.54(\mathrm{td}, J=8.0,5.4 \mathrm{~Hz}, 1 \mathrm{H}), 7.38(\mathrm{tdd}, J=8.2,2.6,1.0 \mathrm{~Hz}, 1 \mathrm{H}), 7.25-7.14(\mathrm{~m}, 4 \mathrm{H})$, $5.39(\mathrm{~d}, J=7.8 \mathrm{~Hz}, 1 \mathrm{H}), 5.16(\mathrm{~d}, J=7.7 \mathrm{~Hz}, 1 \mathrm{H}), 3.20(\mathrm{~s}, 3 \mathrm{H}), 2.32(\mathrm{~s}, 3 \mathrm{H}) .{ }^{13} \mathrm{C}$ NMR $(126$ $\left.\mathrm{MHz}, \mathrm{CDCl}_{3}\right) \delta 184.13,166.61,163.11\left(\mathrm{~d},{ }^{1} J_{\mathrm{C}-\mathrm{F}}=250.6 \mathrm{~Hz}\right), 139.07,137.25\left(\mathrm{~d},{ }^{3} J_{\mathrm{C}-\mathrm{F}}=6.8\right.$ $\mathrm{Hz}), 131.18\left(\mathrm{~d},{ }^{3} J_{\mathrm{C}-\mathrm{F}}=7.8 \mathrm{~Hz}\right), 130.95,130.29,128.58,125.40\left(\mathrm{~d},{ }^{4} J_{\mathrm{C}-\mathrm{F}}=3.0 \mathrm{~Hz}\right), 122.61(\mathrm{~d}$, $\left.{ }^{2} J_{\mathrm{C}-\mathrm{F}}=21.5 \mathrm{~Hz}\right), 116.19\left(\mathrm{~d},{ }^{2} J_{\mathrm{C}-\mathrm{F}}=23.1 \mathrm{~Hz}\right), 70.24,50.14,25.14,21.26 .{ }^{19} \mathrm{~F}$ NMR $(377 \mathrm{MHz}$, $\left.\mathrm{CDCl}_{3}\right) \delta$-110.07. HRMS ESI [M-H] $]^{-}$calculated for $\mathrm{C}_{18} \mathrm{H}_{15} \mathrm{FNO}_{4} \mathrm{~S} 360.0711$, found 360.0718 . 
<smiles>Cc1ccc([C@H]2C(=O)N(C)S(=O)(=O)[C@H]2C(=O)c2ccccc2F)cc1</smiles>

( \pm )-(4R,5R)-5-(2-fluorobenzoyl)-2-methyl-4-(p-tolyl)isothiazolidin-3-one 1,1-dioxide (3p). Following the general procedure, the title compound $(27.1 \mathrm{mg})$ was obtained in $75 \%$ yield. ${ }^{1} \mathrm{H}$ NMR $\left(400 \mathrm{MHz}, \mathrm{CDCl}_{3}\right) \delta 7.98(\mathrm{td}, J=7.7,1.8 \mathrm{~Hz}, 1 \mathrm{H}), 7.65$ (dddd, $J=8.4,7.2,5.2,1.9 \mathrm{~Hz}$, 1H), 7.32 (ddd, $J=8.3,7.4,1.1 \mathrm{~Hz}, 1 \mathrm{H}), 7.29-7.25(\mathrm{~m}, 2 \mathrm{H}), 7.25-7.17(\mathrm{~m}, 3 \mathrm{H}), 5.58(\mathrm{~d}, J$ $=5.0 \mathrm{~Hz}, 1 \mathrm{H}), 5.04(\mathrm{~d}, J=4.8 \mathrm{~Hz}, 1 \mathrm{H}), 3.15(\mathrm{~s}, 3 \mathrm{H}), 2.34(\mathrm{~s}, 3 \mathrm{H}) .{ }^{13} \mathrm{C} \mathrm{NMR}\left(126 \mathrm{MHz}, \mathrm{CDCl}_{3}\right)$ $\delta 184.14,166.36,162.24\left(\mathrm{~d},{ }^{1} J_{\mathrm{C}-\mathrm{F}}=257.0 \mathrm{~Hz}\right), 138.74,137.07\left(\mathrm{~d},{ }^{3} J_{\mathrm{C}-\mathrm{F}}=9.8 \mathrm{~Hz}\right), 131.58$, 131.30, 130.08, 128.26, $125.23\left(\mathrm{~d},{ }^{4} J_{\mathrm{C}-\mathrm{F}}=3.4 \mathrm{~Hz}\right), 123.82\left(\mathrm{~d},{ }^{3} J_{\mathrm{C}-\mathrm{F}}=10.1 \mathrm{~Hz}\right), 117.21\left(\mathrm{~d},{ }^{2} J_{\mathrm{C}-}\right.$ $\mathrm{F}=23.7 \mathrm{~Hz}), 72.86\left(\mathrm{~d},{ }^{4} J_{\mathrm{C}-\mathrm{F}}=8.1 \mathrm{~Hz}\right), 50.18,24.89,21.12 .{ }^{19} \mathrm{~F} \mathrm{NMR}\left(377 \mathrm{MHz}, \mathrm{CDCl}_{3}\right) \delta-$ 108.25. HRMS ESI [M-H] $]^{-}$calculated for $\mathrm{C}_{18} \mathrm{H}_{15} \mathrm{FNO}_{4} \mathrm{~S} 360.0711$, found 360.0718 .

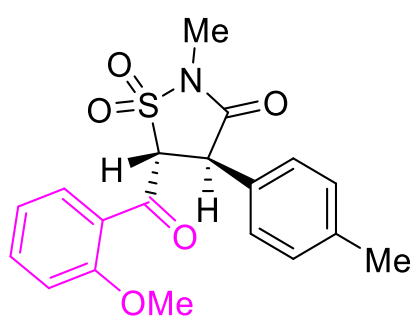

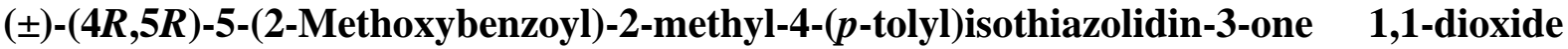
(3q). Following the general procedure, the title compound $(26.9 \mathrm{mg})$ was obtained in $72 \%$ yield. ${ }^{1} \mathrm{H}$ NMR (400 MHz, $\mathrm{CDCl}_{3}$ ) $\delta 7.94(\mathrm{dd}, J=7.9,1.8 \mathrm{~Hz}, 1 \mathrm{H}), 7.60$ (ddd, $J=8.4,7.3,1.8 \mathrm{~Hz}$, $1 \mathrm{H}), 7.27(\mathrm{~d}, J=8.2 \mathrm{~Hz}, 2 \mathrm{H}), 7.18(\mathrm{~d}, J=7.7 \mathrm{~Hz}, 2 \mathrm{H}), 7.07$ (ddd, $J=8.1,7.3,1.0 \mathrm{~Hz}, 1 \mathrm{H})$, $7.03(\mathrm{dd}, J=8.4,0.9 \mathrm{~Hz}, 1 \mathrm{H}), 5.81(\mathrm{~d}, J=3.4 \mathrm{~Hz}, 1 \mathrm{H}), 4.99$ (d, $J=3.4 \mathrm{~Hz}, 1 \mathrm{H}), 3.99(\mathrm{~s}, 3 \mathrm{H})$, $3.14(\mathrm{~s}, 3 \mathrm{H}), 2.33(\mathrm{~s}, 3 \mathrm{H}) .{ }^{13} \mathrm{C}$ NMR $\left(126 \mathrm{MHz}, \mathrm{CDCl}_{3}\right) \delta 186.83,166.54,159.78,138.40$, 136.54, 132.55, 131.81, 129.99, 128.13, 124.97, 121.37, 112.24, 73.67, 55.68, 50.51, 24.84, 21.11. HRMS ESI [M-H] ${ }^{-}$calculated for $\mathrm{C}_{19} \mathrm{H}_{18} \mathrm{NO}_{5} \mathrm{~S} 372.0911$, found 372.0903 .

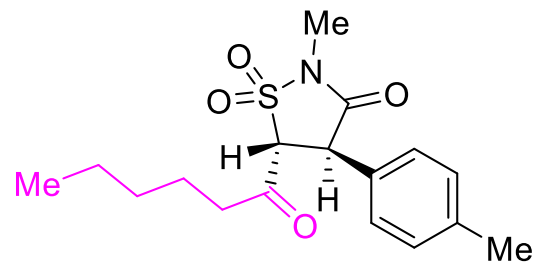

$( \pm)-(4 R, 5 R)-5$-hexanoyl-2-methyl-4-( $p$-tolyl)isothiazolidin-3-one $\quad$ 1,1-dioxide $\quad(3 r)$. Following the general procedure, the title compound $(16.9 \mathrm{mg})$ was obtained in $50 \%$ yield. ${ }^{1} \mathrm{H}$ $\operatorname{NMR}\left(400 \mathrm{MHz}, \mathrm{CDCl}_{3}\right) \delta 7.18(\mathrm{~d}, J=8.1 \mathrm{~Hz}, 2 \mathrm{H}), 7.14(\mathrm{~d}, J=8.3 \mathrm{~Hz}, 2 \mathrm{H}), 4.83(\mathrm{~d}, J=7.6$ $\mathrm{Hz}, 1 \mathrm{H}), 4.66$ (d, $J=7.7 \mathrm{~Hz}, 1 \mathrm{H}), 3.15$ (s, 3H), 2.88 (ddd, $J=18.1,8.3,6.6 \mathrm{~Hz}, 1 \mathrm{H}), 2.61$ (ddd, $J=18.1,8.1,6.0 \mathrm{~Hz}, 1 \mathrm{H}), 2.33(\mathrm{~s}, 3 \mathrm{H}), 1.72-1.59(\mathrm{~m}, 2 \mathrm{H}), 1.37-1.20(\mathrm{~m}, 4 \mathrm{H}), 0.86(\mathrm{t}, J=$ $6.9 \mathrm{~Hz}, 3 \mathrm{H}) .{ }^{13} \mathrm{C} \mathrm{NMR}\left(126 \mathrm{MHz}, \mathrm{CDCl}_{3}\right) \delta 195.13,166.61,138.93,131.01,130.21,128.48$, 73.56, 49.46, 44.81, 30.93, 24.89, 22.93, 22.43, 21.26, 13.93. HRMS ESI [M-H] ${ }^{-}$calculated for $\mathrm{C}_{17} \mathrm{H}_{22} \mathrm{NO}_{4} \mathrm{~S} 336.1275$, found 336.1268 . 
<smiles>Cc1ccc([C@H]2C(=O)N(C)S(=O)(=O)[C@H]2C(=O)CC(C)C)cc1</smiles>

( \pm )-(4R,5R)-2-methyl-5-(3-methylbutanoyl)-4-(p-tolyl)isothiazolidin-3-one 1,1-dioxide (3s). Following the general procedure, the title compound $(17.7 \mathrm{mg})$ was obtained in $55 \%$ yield. ${ }^{1} \mathrm{H}$ NMR $\left(400 \mathrm{MHz}, \mathrm{CDCl}_{3}\right) \delta 7.18(\mathrm{~d}, J=8.1 \mathrm{~Hz}, 2 \mathrm{H}), 7.13(\mathrm{~d}, J=8.3 \mathrm{~Hz}, 2 \mathrm{H}), 4.83(\mathrm{~d}, J=$ $7.7 \mathrm{~Hz}, 1 \mathrm{H}), 4.63(\mathrm{~d}, J=7.7 \mathrm{~Hz}, 1 \mathrm{H}), 3.15(\mathrm{~s}, 3 \mathrm{H}), 2.74(\mathrm{dd}, J=17.5,7.7 \mathrm{~Hz}, 1 \mathrm{H}), 2.55(\mathrm{dd}, J$ $=17.5,5.9 \mathrm{~Hz}, 1 \mathrm{H}), 2.33(\mathrm{~s}, 3 \mathrm{H}), 2.26-2.16(\mathrm{~m}, 1 \mathrm{H}), 0.91(\mathrm{~d}, J=6.7 \mathrm{~Hz}, 6 \mathrm{H}) .{ }^{13} \mathrm{C} \mathrm{NMR}(126$ $\left.\mathrm{MHz}, \mathrm{CDCl}_{3}\right) \delta 194.58,166.62,138.92,131.02,130.23,128.44,73.79,53.54,49.42,24.89$, 24.48, 22.60, 22.12, 21.28. HRMS ESI [M-H] $]^{-}$calculated for $\mathrm{C}_{16} \mathrm{H}_{20} \mathrm{NO}_{4} \mathrm{~S} 322.1119$, found 322.1121 .

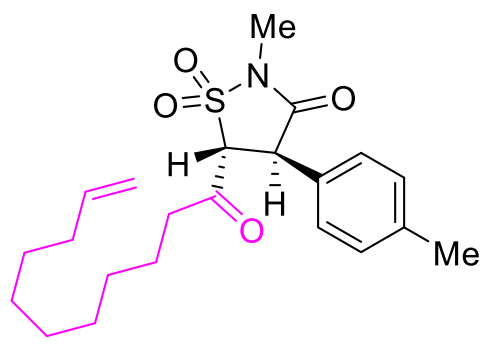

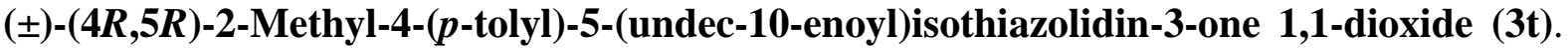
Following the general procedure, the title compound $(24.3 \mathrm{mg})$ was obtained in $60 \%$ yield. ${ }^{1} \mathrm{H}$ $\operatorname{NMR}\left(500 \mathrm{MHz}, \mathrm{CDCl}_{3}\right) \delta 7.18(\mathrm{~d}, J=8.1 \mathrm{~Hz}, 2 \mathrm{H}), 7.14(\mathrm{~d}, J=8.2 \mathrm{~Hz}, 2 \mathrm{H}), 5.89-5.70(\mathrm{~m}$, $1 \mathrm{H}), 4.98(\mathrm{dd}, J=17.1,1.9 \mathrm{~Hz}, 1 \mathrm{H}), 4.93$ (ddd, $J=10.1,2.4,1.2 \mathrm{~Hz}, 1 \mathrm{H}), 4.83$ (d, $J=7.6 \mathrm{~Hz}$, $1 \mathrm{H}), 4.66(\mathrm{~d}, J=7.6 \mathrm{~Hz}, 1 \mathrm{H}), 3.15(\mathrm{~s}, 3 \mathrm{H}), 2.88$ (ddd, $J=18.0,8.4,6.5 \mathrm{~Hz}, 1 \mathrm{H}), 2.61$ (ddd, $J$ $=18.1,8.2,5.9 \mathrm{~Hz}, 1 \mathrm{H}), 2.33(\mathrm{~s}, 3 \mathrm{H}), 2.12-1.94(\mathrm{~m}, 2 \mathrm{H}), 1.36(\mathrm{~d}, J=7.8 \mathrm{~Hz}, 2 \mathrm{H}), 1.34-1.21$ $(\mathrm{m}, 10 \mathrm{H}) .{ }^{13} \mathrm{C}$ NMR $\left(126 \mathrm{MHz}, \mathrm{CDCl}_{3}\right) \delta 195.13,166.60,139.28,138.93,131.04,130.22$, 128.48, 114.31, 73.59, 49.47, 44.85, 33.90, 29.31, 29.31, 29.13, 29.00, 28.80, 24.90, 23.26, 21.27. HRMS ESI [M-H] $]^{-}$calculated for $\mathrm{C}_{22} \mathrm{H}_{30} \mathrm{NO}_{4} \mathrm{~S} 404.1901$, found 404.1912.<smiles>Cc1ccc([C@H]2C(=O)N(C)S(=O)(=O)[C@H]2C(=O)C2CCCCC2)cc1</smiles>

( \pm )-(4R,5R)-5-(Cyclohexanecarbonyl)-2-methyl-4-( $p$-tolyl)isothiazolidin-3-one

dioxide (3u). Following the general procedure, the title compound (16.4 mg) was obtained in $47 \%$ yield. ${ }^{1} \mathrm{H}$ NMR $\left(500 \mathrm{MHz}, \mathrm{CDCl}_{3}\right) \delta 7.17(\mathrm{~d}, J=8.0 \mathrm{~Hz}, 2 \mathrm{H}), 7.11(\mathrm{~d}, J=8.2 \mathrm{~Hz}, 2 \mathrm{H})$, $4.83(\mathrm{~d}, J=7.4 \mathrm{~Hz}, 1 \mathrm{H}), 4.81$ (d, $J=7.4 \mathrm{~Hz}, 1 \mathrm{H}), 3.14$ (s, 3H), 2.65 (tt, $J=11.3,3.4 \mathrm{~Hz}, 1 \mathrm{H})$, $2.33(\mathrm{~s}, 3 \mathrm{H}), 2.01-1.90(\mathrm{~m}, 1 \mathrm{H}), 1.83-1.79(\mathrm{~m}, 2 \mathrm{H}), 1.77-1.71(\mathrm{~m}, 1 \mathrm{H}), 1.69-1.64(\mathrm{~m}$, $1 \mathrm{H}), 1.53-1.44(\mathrm{~m}, 1 \mathrm{H}), 1.34-1.24(\mathrm{~m}, 2 \mathrm{H}), 1.22-1.09(\mathrm{~m}, 2 \mathrm{H}) .{ }^{13} \mathrm{C}$ NMR $(126 \mathrm{MHz}$, $\left.\mathrm{CDCl}_{3}\right) \delta 198.57,166.63,138.87,131.01,130.23,128.27,71.62,52.41,50.02,28.62,26.90$, 
25.67, 25.61, 24.91, 24.89, 21.27. HRMS ESI [M-H] $]^{-}$calculated for $\mathrm{C}_{18} \mathrm{H}_{22} \mathrm{NO}_{4} \mathrm{~S} 348.1275$, found 348.1264 .<smiles>Cc1ccc([C@H]2C(=O)N(C)S(=O)(=O)[C@H]2C(=O)C2CC2)cc1</smiles>

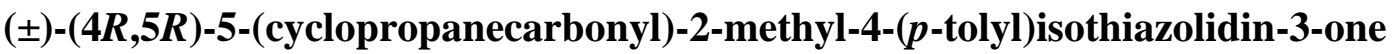
dioxide (3v). Following the general procedure, the title compound $(24.6 \mathrm{mg})$ was obtained in $80 \%$ yield. ${ }^{1} \mathrm{H}$ NMR $\left(400 \mathrm{MHz}, \mathrm{CDCl}_{3}\right) \delta 7.19(\mathrm{~d}, J=8.3 \mathrm{~Hz}, 2 \mathrm{H}), 7.16(\mathrm{~d}, J=8.5 \mathrm{~Hz}, 2 \mathrm{H})$, $4.87(\mathrm{~d}, J=7.9 \mathrm{~Hz}, 1 \mathrm{H}), 4.84(\mathrm{~d}, J=7.9 \mathrm{~Hz}, 1 \mathrm{H}), 3.16(\mathrm{~s}, 3 \mathrm{H}), 2.34(\mathrm{~s}, 3 \mathrm{H}), 2.28-2.17(\mathrm{~m}$, $1 \mathrm{H}), 1.38-1.30(\mathrm{~m}, 1 \mathrm{H}), 1.30-1.20(\mathrm{~m}, 2 \mathrm{H}), 1.17-1.08(\mathrm{~m}, 1 \mathrm{H}) .{ }^{13} \mathrm{C}$ NMR $(126 \mathrm{MHz}$, $\left.\mathrm{CDCl}_{3}\right) \delta 195.32,166.68,138.84,131.11,130.17,128.56,74.69,49.29,24.87,22.89,21.27$, 14.66, 13.81. HRMS ESI [M-H] $]^{-}$calculated for $\mathrm{C}_{15} \mathrm{H}_{16} \mathrm{NO}_{4} \mathrm{~S} 306.0806$, found 306.0809.<smiles>Cc1ccc([C@H]2C(=O)N(C)[S@](=O)[C@H]2C(=O)c2ccc3c(c2)OC(F)(F)O3)cc1</smiles>

$( \pm)-(4 R, 5 R)-5-(2,2-d i f l u o r o b e n z o[d][1,3]$ dioxole-5-carbonyl)-2-methyl-4- $(p$ -

tolyl)isothiazolidin-3-one 1,1-dioxide (3w). Following the general procedure, the title compound $(34.0 \mathrm{mg})$ was obtained in $80 \%$ yield. ${ }^{1} \mathrm{H}$ NMR $\left(400 \mathrm{MHz}, \mathrm{CDCl}_{3}\right) \delta 7.96(\mathrm{dd}, J=$ $8.4,1.8 \mathrm{~Hz}, 1 \mathrm{H}), 7.83(\mathrm{~d}, J=1.8 \mathrm{~Hz}, 1 \mathrm{H}), 7.22(\mathrm{~d}, J=8.4 \mathrm{~Hz}, 1 \mathrm{H}), 7.20-7.16(\mathrm{~m}, 4 \mathrm{H}), 5.35$ $(\mathrm{d}, J=7.9 \mathrm{~Hz}, 1 \mathrm{H}), 5.17(\mathrm{~d}, J=7.9 \mathrm{~Hz}, 1 \mathrm{H}), 3.20(\mathrm{~s}, 3 \mathrm{H}), 2.32(\mathrm{~s}, 3 \mathrm{H}) .{ }^{13} \mathrm{C}$ NMR $(126 \mathrm{MHz}$, $\left.\mathrm{CDCl}_{3}\right) \delta 182.93,166.59,148.80,144.79,139.11,131.92,131.81\left(\mathrm{t},{ }^{1} J_{\mathrm{C}-\mathrm{F}}=259.6 \mathrm{~Hz}\right), 130.90$, 130.30, 128.58, 127.43, 110.36, 109.96, 70.15, 50.14, 25.16, 21.26. ${ }^{19} \mathrm{~F}$ NMR $(377 \mathrm{MHz}$, $\left.\mathrm{CDCl}_{3}\right) \delta$-49.63. HRMS ESI [M-H] $]^{-}$calculated for $\mathrm{C}_{19} \mathrm{H}_{14} \mathrm{~F}_{2} \mathrm{NO}_{6} \mathrm{~S} 422.0515$, found 422.0508 .<smiles>Cc1ccc([C@H]2C(=O)N(C)S(=O)(=O)[C@H]2C(=O)c2cnn(C)c2)cc1</smiles>

( \pm )-(4R,5R)-2-Methyl-5-(1-methyl-1H-pyrazole-4-carbonyl)-4-(p-tolyl)isothiazolidin-3one 1,1-dioxide (3x). Following the general procedure, the title compound (26.1 $\mathrm{mg}$ ) was obtained in $75 \%$ yield. ${ }^{1} \mathrm{H}$ NMR $\left(400 \mathrm{MHz}, \mathrm{CDCl}_{3}\right) \delta 8.08(\mathrm{~s}, 2 \mathrm{H}), 7.23-7.12(\mathrm{~m}, 4 \mathrm{H}), 5.12$ $(\mathrm{d}, J=8.4 \mathrm{~Hz}, 1 \mathrm{H}), 4.99(\mathrm{~d}, J=8.4 \mathrm{~Hz}, 1 \mathrm{H}), 3.95(\mathrm{~s}, 3 \mathrm{H}), 3.18(\mathrm{~s}, 3 \mathrm{H}), 2.32(\mathrm{~s}, 3 \mathrm{H}) .{ }^{13} \mathrm{C} \mathrm{NMR}$ $\left(126 \mathrm{MHz}, \mathrm{CDCl}_{3}\right) \delta 176.98,166.90,141.39,138.92,134.20,131.02,130.22,128.64,123.17$, 72.16, 49.25, 39.84, 25.04, 21.27. HRMS ESI [M-H] $]^{-}$calculated for $\mathrm{C}_{16} \mathrm{H}_{16} \mathrm{~N}_{3} \mathrm{O}_{4} \mathrm{~S} 346.0867$, found 346.0891 . 
<smiles>Cc1ccc([C@H]2C(=O)N(C)S(=O)(=O)[C@H]2C(=O)c2c(C)noc2C)cc1</smiles>

( \pm )-(4R,5R)-5-(3,5-Dimethylisoxazole-4-carbonyl)-2-methyl-4-(p-tolyl)isothiazolidin-3-

one 1,1-dioxide (3y). Following the general procedure, the title compound (24.6 $\mathrm{mg}$ ) was obtained in $68 \%$ yield. ${ }^{1} \mathrm{H}$ NMR $\left(400 \mathrm{MHz}, \mathrm{CDCl}_{3}\right) \delta 7.20(\mathrm{~s}, 4 \mathrm{H}), 5.12(\mathrm{~d}, J=6.6 \mathrm{~Hz}, 1 \mathrm{H})$, $5.02(\mathrm{~d}, J=6.5 \mathrm{~Hz}, 1 \mathrm{H}), 3.16(\mathrm{~s}, 3 \mathrm{H}), 2.75(\mathrm{~s}, 3 \mathrm{H}), 2.50(\mathrm{~s}, 3 \mathrm{H}), 2.34(\mathrm{~s}, 3 \mathrm{H}) .{ }^{13} \mathrm{C} \mathrm{NMR}(126$ $\left.\mathrm{MHz} \mathrm{CDCl}_{3}\right) \delta 179.89,176.20,166.79,158.74,139.40,130.41,130.29,128.29,117.28,71.77$, $50.15,25.08,21.26,14.60,12.41$. HRMS ESI $[\mathrm{M}+\mathrm{H}]^{+}$calculated for $\mathrm{C}_{17} \mathrm{H}_{17} \mathrm{~N}_{2} \mathrm{O}_{5} \mathrm{~S} 361.0864$, found 361.0851 .

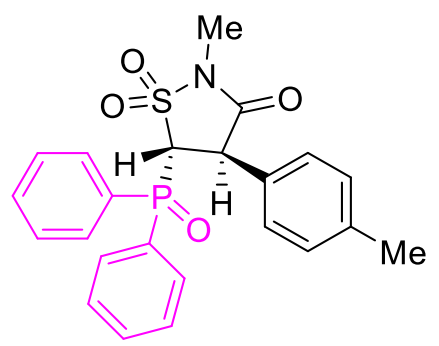

$( \pm)-(4 R, 5 R)-5$-(Diphenylphosphoryl)-2-methyl-4-(p-tolyl)isothiazolidin-3-one 1,1-dioxide (4a). Following the general procedure, the title compound $(30.8 \mathrm{mg})$ was obtained in $70 \%$ yield. ${ }^{1} \mathrm{H}$ NMR $\left(400 \mathrm{MHz}, \mathrm{CDCl}_{3}\right) \delta 8.02-7.86(\mathrm{~m}, 2 \mathrm{H}), 7.68-7.59(\mathrm{~m}, 3 \mathrm{H}), 7.54(\mathrm{tdd}, J=8.0,3.5$, $1.8 \mathrm{~Hz}, 2 \mathrm{H}), 7.48(\mathrm{td}, J=7.4,1.4 \mathrm{~Hz}, 1 \mathrm{H}), 7.34(\mathrm{td}, J=7.7,3.4 \mathrm{~Hz}, 2 \mathrm{H}), 6.94(\mathrm{~d}, J=7.9 \mathrm{~Hz}$, $2 \mathrm{H}), 6.81(\mathrm{~d}, J=8.1 \mathrm{~Hz}, 2 \mathrm{H}), 4.72(\mathrm{dd}, J=14.2,6.5 \mathrm{~Hz}, 1 \mathrm{H}), 4.62(\mathrm{dd}, J=6.5,3.7 \mathrm{~Hz}, 1 \mathrm{H})$, 3.09 (s, 3H), 2.24 (s, 3H). ${ }^{13} \mathrm{C} \mathrm{NMR}\left(126 \mathrm{MHz}, \mathrm{CDCl}_{3}\right) \delta 166.27\left(\mathrm{~d},{ }^{3} J_{\mathrm{C}-\mathrm{P}}=6.8 \mathrm{~Hz}\right.$, carbonyl carbon), 138.59, $133.33\left(\mathrm{~d},{ }^{4} J_{\mathrm{C}-\mathrm{P}}=3.2 \mathrm{~Hz}\right), 132.76\left(\mathrm{~d},{ }^{4} J_{\mathrm{C}-\mathrm{P}}=3.2 \mathrm{~Hz}\right), 131.82\left(\mathrm{~d},{ }^{3} J_{\mathrm{C}-\mathrm{P}}=2.9\right.$ $\mathrm{Hz}$, quaternary carbon on $p$-tolyl), $131.62\left(\mathrm{~d},{ }^{3} J_{\mathrm{C}-\mathrm{P}}=9.7 \mathrm{~Hz}\right), 131.23\left(\mathrm{~d},{ }^{3} J_{\mathrm{C}-\mathrm{P}}=9.3 \mathrm{~Hz}\right)$, $129.89,129.84\left(\mathrm{~d},{ }^{1} J_{\mathrm{C}-\mathrm{P}}=51.7 \mathrm{~Hz}\right), 129.11\left(\mathrm{~d},{ }^{2} J_{\mathrm{C}-\mathrm{P}}=13.1 \mathrm{~Hz}\right), 129.01\left(\mathrm{~d},{ }^{2} J_{\mathrm{C}-\mathrm{P}}=14.0 \mathrm{~Hz}\right)$, $128.99\left(\mathrm{~d},{ }^{1} J_{\mathrm{C}-\mathrm{P}}=53.2 \mathrm{~Hz}\right), 128.13,64.80\left(\mathrm{~d},{ }^{1} J_{\mathrm{C}-\mathrm{P}}=55.9 \mathrm{~Hz}\right), 50.15,24.98,21.16 .{ }^{31} \mathrm{P}$ NMR $\left(162 \mathrm{MHz}, \mathrm{CDCl}_{3}\right) \delta 22.43$. HRMS ESI $[\mathrm{M}-\mathrm{H}]^{-}$calculated for $\mathrm{C}_{23} \mathrm{H}_{21} \mathrm{NO}_{4} \mathrm{PS} 438.0934$, found 438.0938 .

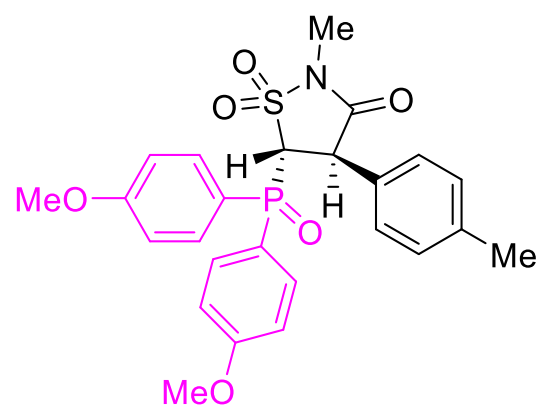

( \pm )-(4R,5R)-5-(Bis(4-methoxyphenyl)phosphoryl)-2-methyl-4-( $p$-tolyl)isothiazolidin-3-

one 1,1-dioxide (4b). Following the general procedure, the title compound (32.0 $\mathrm{mg}$ ) was obtained in $64 \%$ yield. ${ }^{1} \mathrm{H}$ NMR $\left(500 \mathrm{MHz}, \mathrm{CDCl}_{3}\right) \delta 7.86(\mathrm{dd}, J=11.8,8.7 \mathrm{~Hz}, 2 \mathrm{H}), 7.53$ (dd, $J=11.6,8.7 \mathrm{~Hz}, 2 \mathrm{H}), 7.01(\mathrm{dd}, J=8.7,2.8 \mathrm{~Hz}, 2 \mathrm{H}), 6.97(\mathrm{~d}, J=8.0 \mathrm{~Hz}, 2 \mathrm{H}), 6.86(\mathrm{~d}, J=8.2$ 
$\mathrm{Hz}, 2 \mathrm{H}), 6.82(\mathrm{dd}, J=8.7,2.7 \mathrm{~Hz}, 2 \mathrm{H}), 4.67(\mathrm{dd}, J=14.1,6.0 \mathrm{~Hz}, 1 \mathrm{H}), 4.55(\mathrm{dd}, J=5.8,4.9$ $\mathrm{Hz}, 1 \mathrm{H}), 3.84$ (s, 3H), 3.80 (s, 3H), 3.06 (s, 3H), 2.25 (s, 3H). ${ }^{13} \mathrm{C} \mathrm{NMR} \mathrm{(126} \mathrm{MHz,} \mathrm{CDCl} 3$ ) $\delta$ $166.47\left(\mathrm{~d},{ }^{3} J_{\mathrm{C}-\mathrm{P}}=6.3 \mathrm{~Hz}\right.$, carbonyl carbon), $163.53\left(\mathrm{~d},{ }^{4} J_{\mathrm{C}-\mathrm{P}}=2.9 \mathrm{~Hz}\right), 163.19\left(\mathrm{~d},{ }^{4} J_{\mathrm{C}-\mathrm{P}}=2.9\right.$ $\mathrm{Hz}), 138.43,133.83\left(\mathrm{~d},{ }^{3} J_{\mathrm{C}-\mathrm{P}}=11.5 \mathrm{~Hz}\right), 133.23\left(\mathrm{~d},{ }^{3} J_{\mathrm{C}-\mathrm{P}}=11.1 \mathrm{~Hz}\right), 132.03\left(\mathrm{~d},{ }^{3} J_{\mathrm{C}-\mathrm{P}}=2.5\right.$ $\mathrm{Hz}$, quaternary carbon on $p$-tolyl), 129.85, $128.21,120.78$ (d, $\left.{ }^{1} J_{\mathrm{C}-\mathrm{P}}=86.3 \mathrm{~Hz}\right), 119.86\left(\mathrm{~d},{ }^{1} \mathrm{~J}\right.$ $\mathrm{C}-\mathrm{P}=86.8 \mathrm{~Hz}), 114.67\left(\mathrm{~d},{ }^{2} J_{\mathrm{C}-\mathrm{P}}=14.1 \mathrm{~Hz}\right), 114.54\left(\mathrm{~d},{ }^{2} J_{\mathrm{C}-\mathrm{P}}=13.5 \mathrm{~Hz}\right), 65.42\left(\mathrm{~d},{ }^{1} J_{\mathrm{C}-\mathrm{P}}=59.2\right.$ $\mathrm{Hz}), 55.56,55.54,50.30,24.89,21.17 .{ }^{31} \mathrm{P} \mathrm{NMR}\left(162 \mathrm{MHz}, \mathrm{CDCl}_{3}\right) \delta 23.17$. HRMS ESI [M$\mathrm{H}]^{-}$calculated for $\mathrm{C}_{25} \mathrm{H}_{25} \mathrm{NO}_{6} \mathrm{PS} 498.1146$, found 498.1142 .

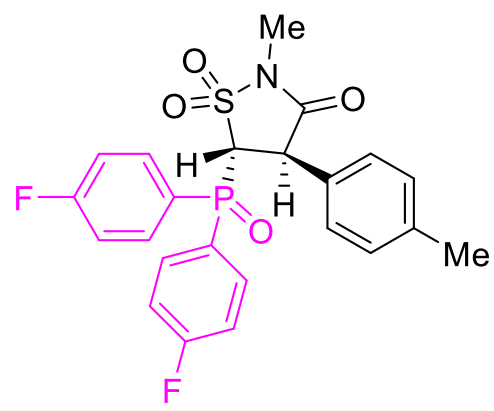

( \pm )-(4R,5R)-5-(Bis(4-fluorophenyl)phosphoryl)-2-methyl-4-(p-tolyl)isothiazolidin-3-one

1,1-dioxide (4c). Following the general procedure, the title compound (31.9 mg) was obtained in $67 \%$ yield. ${ }^{1} \mathrm{H}$ NMR $\left(500 \mathrm{MHz}, \mathrm{CDCl}_{3}\right) \delta 8.04-7.91(\mathrm{~m}, 2 \mathrm{H}), 7.66-7.55(\mathrm{~m}, 2 \mathrm{H}), 7.22(\mathrm{td}$, $J=8.6,2.4 \mathrm{~Hz}, 2 \mathrm{H}), 7.00(\mathrm{td}, J=8.8,2.6 \mathrm{~Hz}, 2 \mathrm{H}), 6.96(\mathrm{~d}, J=8.0 \mathrm{~Hz}, 2 \mathrm{H}), 6.85(\mathrm{~d}, J=8.1$ $\mathrm{Hz}, 2 \mathrm{H}), 4.72(\mathrm{dd}, J=14.0,7.3 \mathrm{~Hz}, 1 \mathrm{H}), 4.63(\mathrm{dd}, J=7.4,3.8 \mathrm{~Hz}, 1 \mathrm{H}), 3.08(\mathrm{~s}, 3 \mathrm{H}), 2.25$ (s, $3 \mathrm{H}) .{ }^{13} \mathrm{C} \mathrm{NMR}\left(126 \mathrm{MHz}, \mathrm{CDCl}_{3}\right) \delta 166.18\left(\mathrm{~d},{ }^{3} J_{\mathrm{C}-\mathrm{P}}=7.8 \mathrm{~Hz}\right.$, carbonyl carbon), 165.94 (dd, $\left.{ }^{1} J_{\mathrm{C}-\mathrm{F}}=257.4 \mathrm{~Hz},{ }^{4} J_{\mathrm{C}-\mathrm{P}}=3.5 \mathrm{~Hz}\right), 165.53\left(\mathrm{dd},{ }^{1} J_{\mathrm{C}-\mathrm{F}}=256.8 \mathrm{~Hz},{ }^{4} J_{\mathrm{C}-\mathrm{P}}=3.4 \mathrm{~Hz}\right), 138.87$, $134.11\left(\mathrm{dd},{ }^{2} J_{\mathrm{C}-\mathrm{F}}=77.5 \mathrm{~Hz},{ }^{3} J_{\mathrm{C}-\mathrm{P}}=9.3 \mathrm{~Hz}\right), 134.02\left(\mathrm{dd},{ }^{2} J_{\mathrm{C}-\mathrm{F}}=77.1 \mathrm{~Hz},{ }^{3} J_{\mathrm{C}-\mathrm{P}}=9.2 \mathrm{~Hz}\right)$, $131.22\left(\mathrm{~d},{ }^{3} J_{\mathrm{C}-\mathrm{P}}=1.9 \mathrm{~Hz}\right.$, quaternary carbon on $p$-tolyl $), 129.93,128.24,125.40\left(\mathrm{dd},{ }^{1} J_{\mathrm{C}-\mathrm{P}}=\right.$ $\left.61.6 \mathrm{~Hz},{ }^{4} J_{\mathrm{C}-\mathrm{F}}=3.7 \mathrm{~Hz}\right), 124.52\left(\mathrm{dd},{ }^{1} J_{\mathrm{C}-\mathrm{P}}=63.5 \mathrm{~Hz},{ }^{4} J_{\mathrm{C}-\mathrm{F}}=3.4 \mathrm{~Hz}\right), 116.72\left(\mathrm{dd},{ }^{3} J_{\mathrm{C}-\mathrm{F}}=\right.$ $\left.21.7 \mathrm{~Hz},{ }^{2} J_{\mathrm{C}-\mathrm{P}}=14.2 \mathrm{~Hz}\right), 116.46\left(\mathrm{dd},{ }^{3} J_{\mathrm{C}-\mathrm{F}}=21.7 \mathrm{~Hz},{ }^{2} J_{\mathrm{C}-\mathrm{P}}=13.7 \mathrm{~Hz}\right), 64.81\left(\mathrm{~d},{ }^{1} J_{\mathrm{C}-\mathrm{P}}=\right.$ $58.2 \mathrm{~Hz}), 50.06,24.96,21.12 .{ }^{19} \mathrm{~F}$ NMR $\left(377 \mathrm{MHz}, \mathrm{CDCl}_{3}\right) \delta-103.59,-104.38 .{ }^{31} \mathrm{P}$ NMR (202 $\left.\mathrm{MHz}, \mathrm{CDCl}_{3}\right) \delta 21.30$. HRMS ESI $[\mathrm{M}-\mathrm{H}]^{-}$calculated for $\mathrm{C}_{23} \mathrm{H}_{19} \mathrm{~F}_{2} \mathrm{NO}_{4} \mathrm{PS}$ 474.0746, found 474.0741 .

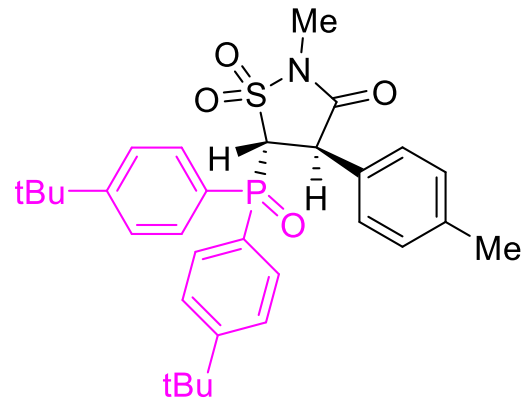

( \pm )-(4R,5R)-5-(Bis(4-(tert-butyl)phenyl)phosphoryl)-2-methyl-4-(p-tolyl)isothiazolidin-3one 1,1-dioxide (4d). Following the general procedure, the title compound (24.3 $\mathrm{mg}$ ) was obtained in $44 \%$ yield. ${ }^{1} \mathrm{H}$ NMR $\left(400 \mathrm{MHz}, \mathrm{CDCl}_{3}\right) \delta 7.89$ (dd, $\left.J=12.2,8.5 \mathrm{~Hz}, 2 \mathrm{H}\right), 7.54$ (ddd, $J=8.5,7.4,4.2 \mathrm{~Hz}, 4 \mathrm{H}), 7.32(\mathrm{dd}, J=8.4,3.1 \mathrm{~Hz}, 2 \mathrm{H}), 6.90(\mathrm{~d}, J=7.5 \mathrm{~Hz}, 2 \mathrm{H}), 6.76(\mathrm{~d}$, $J=8.1 \mathrm{~Hz}, 2 \mathrm{H}), 4.72(\mathrm{dd}, J=14.2,6.8 \mathrm{~Hz}, 1 \mathrm{H}), 4.57(\mathrm{dd}, J=6.8,3.6 \mathrm{~Hz}, 1 \mathrm{H}), 3.09$ (s, 3H), $2.22(\mathrm{~s}, 3 \mathrm{H}), 1.32(\mathrm{~s}, 9 \mathrm{H}), 1.27(\mathrm{~s}, 9 \mathrm{H}) .{ }^{13} \mathrm{C} \mathrm{NMR}\left(126 \mathrm{MHz}, \mathrm{CDCl}_{3}\right) \delta 166.44\left(\mathrm{~d},{ }^{3} J_{\mathrm{C}-\mathrm{P}}=6.3\right.$ 
$\mathrm{Hz}$, carbonyl carbon), $156.91\left(\mathrm{~d},{ }^{4} J_{\mathrm{C}-\mathrm{P}}=3.0 \mathrm{~Hz}\right), 156.52\left(\mathrm{~d},{ }^{4} \mathrm{~J}_{\mathrm{C}-\mathrm{P}}=2.9 \mathrm{~Hz}\right), 138.23,132.02$ $\left(\mathrm{d},{ }^{3} J_{\mathrm{C}-\mathrm{P}}=2.4 \mathrm{~Hz}\right.$, quaternary carbon on $p$-tolyl $), 131.31\left(\mathrm{~d},{ }^{2} J_{\mathrm{C}-\mathrm{P}}=37.7 \mathrm{~Hz}\right), 131.23\left(\mathrm{~d},{ }^{2} J_{\mathrm{C}-}\right.$ $\mathrm{P}=36.9 \mathrm{~Hz}), 129.74,128.15,126.25\left(\mathrm{~d},{ }^{1} J_{\mathrm{C}-\mathrm{P}}=108.4 \mathrm{~Hz}\right), 126.16\left(\mathrm{~d},{ }^{3} J_{\mathrm{C}-\mathrm{P}}=13.2 \mathrm{~Hz}\right), 125.94$ $\left(\mathrm{d},{ }^{1} J_{\mathrm{C}-\mathrm{P}}=110.9 \mathrm{~Hz}\right), 125.92\left(\mathrm{~d},{ }^{3} J_{\mathrm{C}-\mathrm{P}}=12.6 \mathrm{~Hz}\right), 64.90\left(\mathrm{~d},{ }^{1} J_{\mathrm{C}-\mathrm{P}}=55.6 \mathrm{~Hz}\right), 50.10,35.30$, 35.16, 31.17, 31.15, 24.95, 21.21. ${ }^{31} \mathrm{P}$ NMR $\left(202 \mathrm{MHz}, \mathrm{CDCl}_{3}\right) \delta 23.08$. HRMS ESI [M-H] ${ }^{-}$ calculated for $\mathrm{C}_{31} \mathrm{H}_{37} \mathrm{NO}_{4} \mathrm{PS} 550.2186$, found 550.2182.

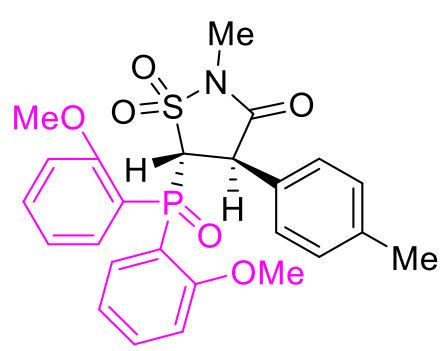

( \pm )-(4R,5R)-5-(Bis(2-methoxyphenyl)phosphoryl)-2-methyl-4-(p-tolyl)isothiazolidin-3-

one 1,1-dioxide (4e). Following the general procedure, the title compound $(29.5 \mathrm{mg})$ was obtained in $59 \%$ yield. ${ }^{1} \mathrm{H}$ NMR $\left(500 \mathrm{MHz}, \mathrm{CDCl}_{3}\right) \delta 8.16$ (ddd, $\left.J=13.0,7.6,1.8 \mathrm{~Hz}, 1 \mathrm{H}\right)$, 7.60 (ddd, $J=15.0,7.7,1.7 \mathrm{~Hz}, 1 \mathrm{H}$ ), 7.54 (dddd, $J=8.2,7.3,1.8,0.8 \mathrm{~Hz}, 1 \mathrm{H}$ ), 7.49 (dddd, $J$ $=8.3,7.3,1.7,0.9 \mathrm{~Hz}, 1 \mathrm{H}), 7.20(\mathrm{tdd}, J=7.5,2.2,0.9 \mathrm{~Hz}, 1 \mathrm{H}), 7.04-6.96(\mathrm{~m}, 3 \mathrm{H}), 6.95(\mathrm{dd}$, $J=8.4,6.4 \mathrm{~Hz}, 1 \mathrm{H}), 6.77(\mathrm{~d}, J=8.1 \mathrm{~Hz}, 2 \mathrm{H}), 6.69(\mathrm{dd}, J=8.3,6.0 \mathrm{~Hz}, 1 \mathrm{H}), 5.19(\mathrm{dd}, J=7.5$, $3.4 \mathrm{~Hz}, 1 \mathrm{H}), 4.56(\mathrm{dd}, J=18.6,3.3 \mathrm{~Hz}, 1 \mathrm{H}), 3.91(\mathrm{~s}, 3 \mathrm{H}), 3.36(\mathrm{~s}, 3 \mathrm{H}), 3.15$ (s, 3H), 2.27 (s, $3 \mathrm{H}) .{ }^{13} \mathrm{C}$ NMR $\left(126 \mathrm{MHz}, \mathrm{CDCl}_{3}\right) \delta 166.36\left(\mathrm{~d},{ }^{3} J_{\mathrm{C}-\mathrm{P}}=6.3 \mathrm{~Hz}\right.$, carbonyl carbon), $159.61\left(\mathrm{~d},{ }^{2} J\right.$ $\mathrm{C}-\mathrm{P}=32.6 \mathrm{~Hz}), 159.57\left(\mathrm{~d},{ }^{2} J_{\mathrm{C}-\mathrm{P}}=33.1 \mathrm{~Hz}\right), 138.09,135.01\left(\mathrm{~d},{ }^{3} J_{\mathrm{C}-\mathrm{P}}=7.6 \mathrm{~Hz}\right), 135.00\left(\mathrm{~d},{ }^{4} J\right.$ $\mathrm{C}-\mathrm{P}=5.2 \mathrm{~Hz}), 134.36\left(\mathrm{~d},{ }^{3} J_{\mathrm{C}-\mathrm{P}}=2.7 \mathrm{~Hz}\right.$, quaternary carbon on $p$-tolyl $), 134.15\left(\mathrm{~d},{ }^{4} J_{\mathrm{C}-\mathrm{P}}=5.2\right.$ $\mathrm{Hz}), 133.66\left(\mathrm{~d},{ }^{3} J_{\mathrm{C}-\mathrm{P}}=7.8 \mathrm{~Hz}\right), 129.82(2 \mathrm{C}), 127.63(2 \mathrm{C}), 121.46\left(\mathrm{~d},{ }^{2} J_{\mathrm{C}-\mathrm{P}}=60.5 \mathrm{~Hz}\right), 121.36$ $\left(\mathrm{d},{ }^{2} J_{\mathrm{C}-\mathrm{P}}=62.0 \mathrm{~Hz}\right), 119.46\left(\mathrm{~d},{ }^{1} J_{\mathrm{C}-\mathrm{P}}=111.6 \mathrm{~Hz}\right), 118.87\left(\mathrm{~d},{ }^{1} J_{\mathrm{C}-\mathrm{P}}=107.7 \mathrm{~Hz}\right), 110.95\left(\mathrm{~d},{ }^{3} J\right.$ $\mathrm{C}-\mathrm{P}=7.4 \mathrm{~Hz}, 2 \mathrm{C}), 65.37\left(\mathrm{~d},{ }^{1} J_{\mathrm{C}-\mathrm{P}}=59.6 \mathrm{~Hz}\right), 55.62,55.08,50.48,25.06,21.21 .{ }^{31} \mathrm{P}$ NMR (162 $\left.\mathrm{MHz}, \mathrm{CDCl}_{3}\right) \delta$ 23.34. HRMS ESI $[\mathrm{M}-\mathrm{H}]^{-}$calculated for $\mathrm{C}_{25} \mathrm{H}_{25} \mathrm{NO}_{6} \mathrm{PS}$ 498.1146, found 498.1141 .<smiles>CN1C(=O)[C@H](c2ccccc2)[C@H](C(=O)c2ccccc2)S1(=O)=O</smiles>

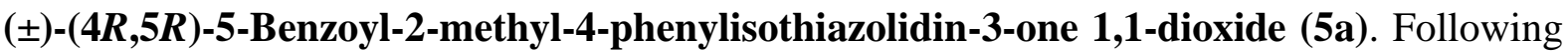
the general procedure, the title compound $(17.8 \mathrm{mg})$ was obtained in $54 \%$ yield. ${ }^{1} \mathrm{H}$ NMR (500 $\left.\mathrm{MHz}, \mathrm{CDCl}_{3}\right) \delta 8.12(\mathrm{dd}, J=8.5,1.3 \mathrm{~Hz}, 2 \mathrm{H}), 7.69$ (tt, $\left.J=7.3,1.3 \mathrm{~Hz}, 1 \mathrm{H}\right), 7.55$ (dd, $J=8.5$, $7.3 \mathrm{~Hz}, 2 \mathrm{H}), 7.42-7.34(\mathrm{~m}, 2 \mathrm{H}), 7.37-7.30(\mathrm{~m}, 3 \mathrm{H}), 5.48(\mathrm{~d}, J=7.7 \mathrm{~Hz}, 1 \mathrm{H}), 5.23(\mathrm{~d}, J=$ $7.6 \mathrm{~Hz}, 1 \mathrm{H}), 3.20$ (s, 3H). ${ }^{13} \mathrm{C}$ NMR $\left(126 \mathrm{MHz}, \mathrm{CDCl}_{3}\right) \delta 184.96,166.61,135.46,135.31$, 134.27, 129.59 (4C), 129.43 (2C), 128.98, 128.75 (2C), 69.96, 50.48, 25.13. HRMS ESI [M$\mathrm{H}]^{-}$calculated for $\mathrm{C}_{17} \mathrm{H}_{14} \mathrm{NO}_{4} \mathrm{~S} 328.0649$, found 328.0641 . 
<smiles>C[C@H]1N(C)C(=O)[C@H](c2ccccc2)[C@@H](C(=O)C2CC2)S1(=O)=O</smiles>

( \pm )-(4R,5R)-5-(Cyclopropanecarbonyl)-2-methyl-4-phenylisothiazolidin-3-one

dioxide (5b). Following the general procedure, the title compound $(23.8 \mathrm{mg})$ was obtained in $81 \%$ yield. ${ }^{1} \mathrm{H}$ NMR $\left(400 \mathrm{MHz}, \mathrm{CDCl}_{3}\right) \delta 7.30-7.23(\mathrm{~m}, 3 \mathrm{H}), 7.19(\mathrm{~d}, J=2.0 \mathrm{~Hz}, 1 \mathrm{H}), 7.17$ $-7.16(\mathrm{~m}, 1 \mathrm{H}), 4.80(\mathrm{~d}, J=7.9 \mathrm{~Hz}, 1 \mathrm{H}), 4.77(\mathrm{~d}, J=8.0 \mathrm{~Hz}, 1 \mathrm{H}), 3.06(\mathrm{~s}, 3 \mathrm{H}), 2.17-2.10(\mathrm{~m}$, $1 \mathrm{H}), 1.28-1.21(\mathrm{~m}, 1 \mathrm{H}), 1.18-1.12(\mathrm{~m}, 2 \mathrm{H}), 1.06-0.98(\mathrm{~m}, 1 \mathrm{H}) .{ }^{13} \mathrm{C}$ NMR $(126 \mathrm{MHz}$, $\left.\mathrm{CDCl}_{3}\right) \delta 195.29,166.46,134.15,129.49,128.86,128.70,74.58,49.55,24.88,22.89,14.72$, 13.88. HRMS ESI [M-H] $]^{-}$calculated for $\mathrm{C}_{14} \mathrm{H}_{14} \mathrm{NO}_{4} \mathrm{~S} 292.0649$, found 292.0641 .<smiles>COc1ccc([C@H]2C(=O)N(C)S(=O)(=O)[C@H]2C(=O)c2ccccc2)cc1</smiles>

$( \pm)-(4 R, 5 R)-5-B e n z o y l-4-(4-m e t h o x y p h e n y l)-2-m e t h y l i s o t h i a z o l i d i n-3-o n e \quad$ 1,1-dioxide (5c). Following the general procedure, the title compound $(22.3 \mathrm{mg})$ was obtained in $62 \%$ yield. ${ }^{1} \mathrm{H}$ NMR $\left(500 \mathrm{MHz}, \mathrm{CDCl}_{3}\right) \delta 8.14-8.09(\mathrm{~m}, 2 \mathrm{H}), 7.72-7.65(\mathrm{~m}, 1 \mathrm{H}), 7.59-7.51(\mathrm{~m}, 2 \mathrm{H})$, $7.25(\mathrm{~d}, J=8.7 \mathrm{~Hz}, 2 \mathrm{H}), 6.89$ (d, $J=8.7 \mathrm{~Hz}, 2 \mathrm{H}), 5.44(\mathrm{~d}, J=7.8 \mathrm{~Hz}, 1 \mathrm{H}), 5.17(\mathrm{~d}, J=7.8 \mathrm{~Hz}$, $1 \mathrm{H}), 3.77$ (s, 3H), 3.19 (s, 3H). ${ }^{13} \mathrm{C} \mathrm{NMR}\left(126 \mathrm{MHz}, \mathrm{CDCl}_{3}\right) \delta 185.04,166.98,160.00,135.43$, $135.32,129.95,129.57,129.41,126.04,114.98,70.02$, 55.48, 49.86, 25.09. HRMS ESI [M$\mathrm{H}]^{-}$calculated for $\mathrm{C}_{18} \mathrm{H}_{16} \mathrm{NO}_{5} \mathrm{~S} 358.0755$, found 358.0748 .<smiles>COc1ccc([C@H]2C(=O)N(C)S(=O)(=O)[C@H]2C(=O)C2CC2)cc1</smiles>

( \pm )-(4R,5R)-5-(Cyclopropanecarbonyl)-4-(4-methoxyphenyl)-2-methylisothiazolidin-3-

one 1,1-dioxide (5d). Following the general procedure, the title compound $(29.1 \mathrm{mg}$ ) was obtained in $90 \%$ yield. ${ }^{1} \mathrm{H}$ NMR $\left(500 \mathrm{MHz}, \mathrm{CDCl}_{3}\right) \delta 7.19(\mathrm{~d}, J=8.8 \mathrm{~Hz}, 2 \mathrm{H}), 6.89(\mathrm{~d}, J=8.7$ $\mathrm{Hz}, 2 \mathrm{H}), 4.85(\mathrm{~d}, J=8.0 \mathrm{~Hz}, 1 \mathrm{H}), 4.82(\mathrm{~d}, J=8.0 \mathrm{~Hz}, 1 \mathrm{H}), 3.79(\mathrm{~s}, 3 \mathrm{H}), 3.15(\mathrm{~s}, 3 \mathrm{H}), 2.23(\mathrm{tt}$, $J=7.4,4.8 \mathrm{~Hz}, 1 \mathrm{H}), 1.37-1.30(\mathrm{~m}, 1 \mathrm{H}), 1.28-1.22(\mathrm{~m}, 2 \mathrm{H}), 1.17-1.06(\mathrm{~m}, 1 \mathrm{H}) .{ }^{13} \mathrm{C} \mathrm{NMR}$ $\left(126 \mathrm{MHz}, \mathrm{CDCl}_{3}\right) \delta 195.39,166.83,159.92,129.90,125.96,114.88,74.68,55.47,48.95$, 24.84, 22.89, 14.65, 13.80. HRMS ESI [M-H] ${ }^{-}$calculated for $\mathrm{C}_{15} \mathrm{H}_{16} \mathrm{NO}_{5} \mathrm{~S} 322.0755$, found 322.0758 .<smiles>CN1C(=O)[C@H](c2ccc(C(C)(C)C)cc2)[C@H](C(=O)C2CC2)S1(=O)=O</smiles> 
( \pm )-(4R,5R)-4-(4-(tert-Butyl)phenyl)-5-(cyclopropanecarbonyl)-2-methylisothiazolidin-3one 1,1-dioxide (5e). Following the general procedure, the title compound (29.1 $\mathrm{mg}$ ) was obtained in $83 \%$ yield. ${ }^{1} \mathrm{H} \mathrm{NMR}\left(400 \mathrm{MHz}, \mathrm{CDCl}_{3}\right) \delta 7.39(\mathrm{~d}, J=8.4 \mathrm{~Hz}, 2 \mathrm{H}), 7.20(\mathrm{~d}, J=8.4$ $\mathrm{Hz}, 2 \mathrm{H}), 4.90(\mathrm{~d}, J=7.7 \mathrm{~Hz}, 1 \mathrm{H}), 4.86(\mathrm{~d}, J=7.7 \mathrm{~Hz}, 1 \mathrm{H}), 3.16(\mathrm{~s}, 3 \mathrm{H}), 2.29-2.18(\mathrm{~m}, 1 \mathrm{H})$, $1.34-1.31(\mathrm{~m}, 1 \mathrm{H}), 1.30(\mathrm{~s}, 9 \mathrm{H}), 1.29-1.23(\mathrm{~m}, 2 \mathrm{H}), 1.15-1.09(\mathrm{~m}, 1 \mathrm{H}) .{ }^{13} \mathrm{C}$ NMR $(126$ $\left.\mathrm{MHz}, \mathrm{CDCl}_{3}\right) \delta 195.40,166.69,151.88,131.09,128.30,126.47,74.58,49.15,34.75,31.34$, 24.86, 22.89, 14.74, 13.82. HRMS ESI [M-H] ${ }^{-}$calculated for $\mathrm{C}_{18} \mathrm{H}_{22} \mathrm{NO}_{4} \mathrm{~S} 348.1275$, found 348.1268 .<smiles>CC(=O)Nc1ccc([C@H]2C(=O)N(C)S(=O)(=O)[C@H]2C(=O)C2CC2)cc1</smiles>

$N$-(4-(( \pm )-(4R,5R)-5-(Cyclopropanecarbonyl)-2-methyl-1,1-dioxido-3-oxoisothiazolidin-

4-yl)phenyl)acetamide (5f). Following the general procedure, the title compound (24.9 $\mathrm{mg}$ ) was obtained in $71 \%$ yield. ${ }^{1} \mathrm{H}$ NMR $\left(500 \mathrm{MHz}, \mathrm{CDCl}_{3}\right) \delta 7.48(\mathrm{~s}, 1 \mathrm{H}), 7.46(\mathrm{~d}, J=8.7 \mathrm{~Hz}$, 2H), $7.19(\mathrm{~d}, J=8.6 \mathrm{~Hz}, 2 \mathrm{H}), 4.87(\mathrm{~d}, J=8.0 \mathrm{~Hz}, 1 \mathrm{H}), 4.83(\mathrm{~d}, J=8.0 \mathrm{~Hz}, 1 \mathrm{H}), 3.16(\mathrm{~s}, 3 \mathrm{H})$, $2.30-2.19(\mathrm{~m}, 1 \mathrm{H}), 2.15(\mathrm{~s}, 3 \mathrm{H}), 1.38-1.30(\mathrm{~m}, 1 \mathrm{H}), 1.30-1.22(\mathrm{~m}, 2 \mathrm{H}), 1.17-1.09(\mathrm{~m}$, 1H). ${ }^{13} \mathrm{C} \mathrm{NMR}\left(126 \mathrm{MHz}, \mathrm{CDCl}_{3}\right) \delta 195.30,168.63,166.67,138.43,129.59,129.41,120.83$, 74.56, 49.10, 24.92, 24.65, 22.92, 14.76, 13.96. HRMS ESI [M-H] $]^{-}$calculated for $\mathrm{C}_{16} \mathrm{H}_{17} \mathrm{~N}_{2} \mathrm{O}_{5} \mathrm{~S} 349.0864$, found 349.0858.<smiles>COC(=O)c1ccc([C@H]2C(=O)N(C)S(=O)(=O)[C@H]2C(=O)C2CC2)cc1</smiles>

Methyl

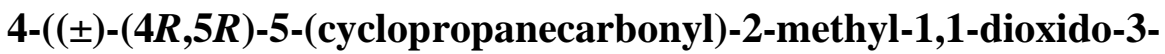
oxoisothiazolidin-4-yl)benzoate (5g). Following the general procedure, the title compound $(23.9 \mathrm{mg})$ was obtained in $68 \%$ yield. ${ }^{1} \mathrm{H}$ NMR $\left(400 \mathrm{MHz}, \mathrm{CDCl}_{3}\right) \delta 8.04(\mathrm{~d}, J=8.4 \mathrm{~Hz}, 2 \mathrm{H})$, $7.36(\mathrm{~d}, J=8.4 \mathrm{~Hz}, 2 \mathrm{H}), 4.94(\mathrm{~d}, J=8.0 \mathrm{~Hz}, 1 \mathrm{H}), 4.88(\mathrm{~d}, J=8.0 \mathrm{~Hz}, 1 \mathrm{H}), 3.91(\mathrm{~s}, 3 \mathrm{H}), 3.17$ (s, 3H), $2.29-2.20(\mathrm{~m}, 1 \mathrm{H}), 1.40-1.32(\mathrm{~m}, 1 \mathrm{H}), 1.31-1.22(\mathrm{~m}, 2 \mathrm{H}), 1.18-1.10(\mathrm{~m}, 1 \mathrm{H})$. ${ }^{13} \mathrm{C} \mathrm{NMR}\left(126 \mathrm{MHz}, \mathrm{CDCl}_{3}\right) \delta 195.06,166.42,165.81,138.80,130.73,130.67,128.91,74.35$, $52.45,49.29,25.00,22.89,14.88,14.07$. HRMS ESI $[\mathrm{M}-\mathrm{H}]^{-}$calculated for $\mathrm{C}_{16} \mathrm{H}_{16} \mathrm{NO}_{6} \mathrm{~S}$ 350.0704 , found 350.0701 .<smiles>CN1C(=O)[C@H](c2ccc(C(F)(F)F)cc2)[C@@H](C(=O)C2CC2)S1(=O)=O</smiles>

$( \pm)-(4 R, 5 R)-5-(C y c l o p r o p a n e c a r b o n y l)-2-m e t h y l-4-(4-$

(trifluoromethyl)phenyl)isothiazolidin-3-one 1,1-dioxide (5h). Following the general 
procedure, the title compound $(27.1 \mathrm{mg})$ was obtained in $75 \%$ yield. ${ }^{1} \mathrm{H}$ NMR $(400 \mathrm{MHz}$, $\left.\mathrm{CDCl}_{3}\right) \delta 7.65(\mathrm{~d}, J=8.0 \mathrm{~Hz}, 2 \mathrm{H}), 7.42(\mathrm{~d}, J=7.9 \mathrm{~Hz}, 2 \mathrm{H}), 4.95(\mathrm{~d}, J=8.1 \mathrm{~Hz}, 1 \mathrm{H}), 4.87(\mathrm{~d}$, $J=8.1 \mathrm{~Hz}, 1 \mathrm{H}), 3.18(\mathrm{~s}, 3 \mathrm{H}), 2.29-2.21(\mathrm{~m}, 1 \mathrm{H}), 1.40-1.33(\mathrm{~m}, 1 \mathrm{H}), 1.33-1.22(\mathrm{~m}, 2 \mathrm{H})$, $1.19-1.12(\mathrm{~m}, 1 \mathrm{H}) .{ }^{13} \mathrm{C} \mathrm{NMR}\left(126 \mathrm{MHz}, \mathrm{CDCl}_{3}\right) \delta 195.00,165.73,137.90,131.26\left(\mathrm{q},{ }^{2} J_{\mathrm{C}-\mathrm{F}}\right.$ $=32.8 \mathrm{~Hz}), 129.36,126.48\left(\mathrm{q},{ }^{3} J_{\mathrm{C}-\mathrm{F}}=3.7 \mathrm{~Hz}\right), 123.85\left(\mathrm{q},{ }^{1} J_{\mathrm{C}-\mathrm{F}}=272.9 \mathrm{~Hz}\right), 74.41,49.13$, 25.04, 22.88, 14.98, 14.12. ${ }^{19} \mathrm{~F}$ NMR $\left(376 \mathrm{MHz}, \mathrm{CDCl}_{3}\right) \delta-62.88$. HRMS ESI [M-H] ${ }^{-}$ calculated for $\mathrm{C}_{15} \mathrm{H}_{13} \mathrm{~F}_{3} \mathrm{NO}_{4} \mathrm{~S} 360.0523$, found 360.0528 .<smiles>C[C@H]1N(C)C(=O)[C@H](c2ccc(F)cc2)[C@@H](C(=O)C2CC2)S1(=O)=O</smiles>

( \pm )-(4R,5R)-5-(Cyclopropanecarbonyl)-4-(4-fluorophenyl)-2-methylisothiazolidin-3-one 1,1-dioxide (5i). Following the general procedure, the title compound (29.9 $\mathrm{mg})$ was obtained in $96 \%$ yield. ${ }^{1} \mathrm{H}$ NMR $\left(400 \mathrm{MHz}, \mathrm{CDCl}_{3}\right) \delta 7.30-7.24(\mathrm{~m}, 2 \mathrm{H}), 7.11-7.03(\mathrm{~m}, 2 \mathrm{H}), 4.87(\mathrm{~d}$, $J=8.0 \mathrm{~Hz}, 1 \mathrm{H}), 4.84(\mathrm{~d}, J=8.0 \mathrm{~Hz}, 1 \mathrm{H}), 3.17(\mathrm{~s}, 3 \mathrm{H}), 2.30-2.20(\mathrm{~m}, 1 \mathrm{H}), 1.41-1.32(\mathrm{~m}$, $1 \mathrm{H}), 1.32-1.24(\mathrm{~m}, 2 \mathrm{H}), 1.20-1.10(\mathrm{~m}, 1 \mathrm{H}) .{ }^{13} \mathrm{C} \mathrm{NMR}\left(126 \mathrm{MHz}, \mathrm{CDCl}_{3}\right) \delta 195.00,165.73$, 137.90, $131.26\left(\mathrm{q},{ }^{2} J_{\mathrm{C}-\mathrm{F}}=32.8 \mathrm{~Hz}\right), 129.36,126.48\left(\mathrm{q},{ }^{3} J_{\mathrm{C}-\mathrm{F}}=3.7 \mathrm{~Hz}\right), 123.85\left(\mathrm{q},{ }^{1} J_{\mathrm{C}-\mathrm{F}}=\right.$ $272.9 \mathrm{~Hz}), 74.41,49.13,25.04,22.88,14.98,14.12 .{ }^{13} \mathrm{C} \mathrm{NMR}\left(126 \mathrm{MHz}, \mathrm{CDCl}_{3}\right) \delta 195.21$, $166.32,162.87\left(\mathrm{~d},{ }^{1} J_{\mathrm{C}-\mathrm{F}}=249.0 \mathrm{~Hz}\right), 130.61\left(\mathrm{~d},{ }^{3} J_{\mathrm{C}-\mathrm{F}}=8.4 \mathrm{~Hz}\right), 129.82\left(\mathrm{~d},{ }^{4} J_{\mathrm{C}-\mathrm{F}}=3.4 \mathrm{~Hz}\right)$, $116.51\left(\mathrm{~d},{ }^{2} J_{\mathrm{C}-\mathrm{F}}=21.5 \mathrm{~Hz}\right), 74.60,48.78,24.91,22.88,14.81,13.96 .{ }^{19} \mathrm{~F}$ NMR $(376 \mathrm{MHz}$, $\left.\mathrm{CDCl}_{3}\right) \delta$-112.50. HRMS ESI [M-H] $]^{-}$calculated for $\mathrm{C}_{14} \mathrm{H}_{13} \mathrm{FNO}_{4} \mathrm{~S} 310.0555$, found 310.0558 .<smiles>CN1C(=O)[C@H](c2cccc(F)c2)[C@@H](C(=O)C2CC2)S1(=O)=O</smiles>

( \pm )-(4R,5R)-5-(Cyclopropanecarbonyl)-4-(3-fluorophenyl)-2-methylisothiazolidin-3-one 1,1-dioxide (5j). Following the general procedure, the title compound 5j (16.2 $\mathrm{mg}$ ) was obtained in $52 \%$ yield. ${ }^{1} \mathrm{H}$ NMR $\left(400 \mathrm{MHz}, \mathrm{CDCl}_{3}\right) \delta 7.35(\mathrm{td}, J=8.0,5.9 \mathrm{~Hz}, 1 \mathrm{H}), 7.09-$ $7.00(\mathrm{~m}, 3 \mathrm{H}), 4.89-4.85(\mathrm{~m}, 2 \mathrm{H}), 3.17(\mathrm{~s}, 3 \mathrm{H}), 2.31-2.19(\mathrm{~m}, 1 \mathrm{H}), 1.42-1.32(\mathrm{~m}, 1 \mathrm{H}), 1.31$ - $1.24(\mathrm{~m}, 2 \mathrm{H}), 1.20-1.09(\mathrm{~m}, 1 \mathrm{H}) .{ }^{13} \mathrm{C} \mathrm{NMR}\left(126 \mathrm{MHz}, \mathrm{CDCl}_{3}\right) \delta 195.07,165.82,163.04$ $\left(\mathrm{d},{ }^{1} J_{\mathrm{C}-\mathrm{F}}=248.5 \mathrm{~Hz}\right), 136.15\left(\mathrm{~d},{ }^{3} J_{\mathrm{C}-\mathrm{F}}=7.6 \mathrm{~Hz}\right), 131.07\left(\mathrm{~d},{ }^{3} J_{\mathrm{C}-\mathrm{F}}=8.4 \mathrm{~Hz}\right), 124.43\left(\mathrm{~d},{ }^{4} J_{\mathrm{C}-\mathrm{F}}\right.$ $=3.2 \mathrm{~Hz}), 115.97\left(2 \mathrm{C}, \mathrm{d},{ }^{2} J_{\mathrm{C}-\mathrm{F}}=21.5 \mathrm{~Hz}\right), 74.33,49.02\left(\mathrm{~d},{ }^{4} J_{\mathrm{C}-\mathrm{F}}=1.9 \mathrm{~Hz}\right), 24.92,22.85$, 14.82, 13.99. ${ }^{19} \mathrm{~F}$ NMR (376 MHz, $\left.\mathrm{CDCl}_{3}\right) \delta-110.97$. HRMS ESI $[\mathrm{M}-\mathrm{H}]^{-}$calculated for $\mathrm{C}_{14} \mathrm{H}_{13} \mathrm{FNO}_{4} \mathrm{~S} 310.0555$, found 310.0552 .<smiles>COc1cccc([C@H]2C(=O)N(C)S(=O)(=O)[C@H]2C(=O)C2CC2)c1</smiles>

( \pm )-(4R,5R)-5-(Cyclopropanecarbonyl)-4-(3-methoxyphenyl)-2-methylisothiazolidin-3one 1,1-dioxide (5k). Following the general procedure, the title compound (24.3 $\mathrm{mg}$ ) was 
obtained in $75 \%$ yield. ${ }^{1} \mathrm{H}$ NMR $\left(400 \mathrm{MHz}, \mathrm{CDCl}_{3}\right) \delta 7.29(\mathrm{dd}, J=8.3,7.6 \mathrm{~Hz}, 1 \mathrm{H}), 6.88$ (ddd, $J=8.4,2.5,0.9 \mathrm{~Hz}, 1 \mathrm{H}), 6.85(\mathrm{ddd}, J=7.7,1.8,0.9 \mathrm{~Hz}, 1 \mathrm{H}), 6.82-6.80(\mathrm{~m}, 1 \mathrm{H}), 4.90(\mathrm{~d}, J=$ $7.7 \mathrm{~Hz}, 1 \mathrm{H}), 4.84(\mathrm{~d}, J=7.7 \mathrm{~Hz}, 1 \mathrm{H}), 3.80(\mathrm{~s}, 3 \mathrm{H}), 3.16(\mathrm{~s}, 3 \mathrm{H}), 2.34-2.15(\mathrm{~m}, 1 \mathrm{H}), 1.44-$ $1.32(\mathrm{~m}, 1 \mathrm{H}), 1.29-1.20(\mathrm{~m}, 2 \mathrm{H}), 1.19-1.07(\mathrm{~m}, 1 \mathrm{H}) .{ }^{13} \mathrm{C} \mathrm{NMR}\left(126 \mathrm{MHz}, \mathrm{CDCl}_{3}\right) \delta 195.30$, $166.28,160.25,135.51,130.55,120.66,114.61,114.24,74.47,55.45,49.52,24.87,22.90$, 14.70, 13.88. HRMS ESI [M-H] $]^{-}$calculated for $\mathrm{C}_{15} \mathrm{H}_{16} \mathrm{NO}_{5} \mathrm{~S} 322.0755$, found 322.0748.

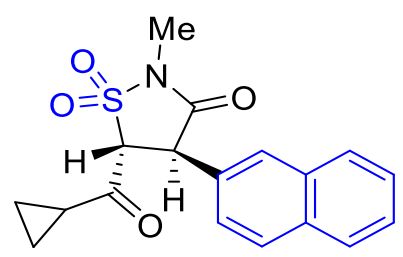

( \pm )-(4R,5R)-5-(Cyclopropanecarbonyl)-2-methyl-4-(naphthalen-2-yl)isothiazolidin-3-one 1,1-dioxide (5l). Following the general procedure, the title compound (18.2 $\mathrm{mg}$ ) was obtained in $53 \%$ yield. ${ }^{1} \mathrm{H}$ NMR $\left(500 \mathrm{MHz}, \mathrm{CDCl}_{3}\right) \delta 7.87(\mathrm{~d}, J=8.5 \mathrm{~Hz}, 1 \mathrm{H}), 7.86-7.80(\mathrm{~m}, 2 \mathrm{H}), 7.78$ $(\mathrm{d}, J=1.8 \mathrm{~Hz}, 1 \mathrm{H}), 7.56-7.48(\mathrm{~m}, 2 \mathrm{H}), 7.32(\mathrm{dd}, J=8.5,1.7 \mathrm{~Hz}, 1 \mathrm{H}), 5.07(\mathrm{~d}, J=8.0 \mathrm{~Hz}$, $1 \mathrm{H}), 4.99(\mathrm{~d}, J=8.0 \mathrm{~Hz}, 1 \mathrm{H}), 3.21(\mathrm{~s}, 3 \mathrm{H}), 2.32-2.18(\mathrm{~m}, 1 \mathrm{H}), 1.42-1.34(\mathrm{~m}, 1 \mathrm{H}), 1.31-$ $1.21(\mathrm{~m}, 2 \mathrm{H}), 1.17-1.06(\mathrm{~m}, 1 \mathrm{H}) .{ }^{13} \mathrm{C}$ NMR $\left(126 \mathrm{MHz}, \mathrm{CDCl}_{3}\right) \delta 195.23,166.52,133.43$, 133.20, 131.29, 129.63, 128.79, 128.13, 127.86, 126.98, 126.94, 125.27, 74.64, 49.77, 24.97, 22.95, 14.75, 13.93. HRMS ESI [M-H] $]^{-}$calculated for $\mathrm{C}_{18} \mathrm{H}_{16} \mathrm{NO}_{4} \mathrm{~S} 342.0806$, found 342.0802.<smiles>COc1ccccc1[C@H]1C(=O)N(C)S(=O)(=O)[C@H]1C(=O)C1CC1</smiles>

$( \pm)-(4 R, 5 R)-5$-(Cyclopropanecarbonyl)-4-(2-methoxyphenyl)-2-methylisothiazolidin-3one 1,1-dioxide (5m). Following the general procedure, the title compound $(21.1 \mathrm{mg})$ was obtained in $65 \%$ yield. ${ }^{1} \mathrm{H}$ NMR $\left(500 \mathrm{MHz}, \mathrm{CDCl}_{3}\right) \delta 7.34(\mathrm{td}, J=7.9,1.7 \mathrm{~Hz}, 1 \mathrm{H}), 7.28$ (dd, $J=7.5,1.7 \mathrm{~Hz}, 1 \mathrm{H}), 6.96(\mathrm{td}, J=7.5,1.1 \mathrm{~Hz}, 1 \mathrm{H}), 6.91(\mathrm{dd}, J=8.2,1.0 \mathrm{~Hz}, 1 \mathrm{H}), 5.05(\mathrm{~d}, J=$ $8.4 \mathrm{~Hz}, 1 \mathrm{H}), 4.77(\mathrm{~d}, J=8.4 \mathrm{~Hz}, 1 \mathrm{H}), 3.84(\mathrm{~s}, 3 \mathrm{H}), 3.16(\mathrm{~s}, 3 \mathrm{H}), 2.22(\mathrm{tt}, J=7.1,4.8 \mathrm{~Hz}, 1 \mathrm{H})$, $1.40-1.30(\mathrm{~m}, 1 \mathrm{H}), 1.29-1.20(\mathrm{~m}, 2 \mathrm{H}), 1.16-1.01(\mathrm{~m}, 1 \mathrm{H}) .{ }^{13} \mathrm{C} \mathrm{NMR}\left(126 \mathrm{MHz}, \mathrm{CDCl}_{3}\right) \delta$ $195.80,166.80,156.67,132.53,130.56,122.35,121.48,111.38,72.52,55.77,47.31,24.65$, 22.70, 14.55, 13.56. HRMS ESI [M-H] $]^{-}$calculated for $\mathrm{C}_{15} \mathrm{H}_{16} \mathrm{NO}_{5} \mathrm{~S} 322.0755$, found 322.0752 .<smiles>C[C@H]1N(C)C(=O)[C@H](c2cccs2)[C@H](C(=O)C2CC2)S1(=O)=O</smiles>

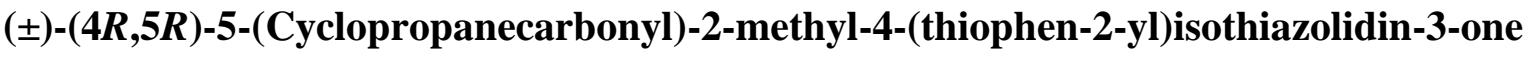

1,1-dioxide (5n). Following the general procedure, the title compound $(26.3 \mathrm{mg})$ was obtained in $88 \%$ yield. ${ }^{1} \mathrm{H}$ NMR $\left(400 \mathrm{MHz}, \mathrm{CDCl}_{3}\right) \delta 7.30(\mathrm{dd}, J=5.1,1.3 \mathrm{~Hz}, 1 \mathrm{H}), 7.04(\mathrm{dt}, J=3.4$, $1.0 \mathrm{~Hz}, 1 \mathrm{H}), 6.99$ (dd, $J=5.1,3.6 \mathrm{~Hz}, 1 \mathrm{H}), 5.15(\mathrm{~d}, J=7.9 \mathrm{~Hz}, 1 \mathrm{H}), 4.94(\mathrm{~d}, J=7.9 \mathrm{~Hz}, 1 \mathrm{H})$, $3.16(\mathrm{~s}, 3 \mathrm{H}), 2.27(\mathrm{tt}, J=7.6,4.5 \mathrm{~Hz}, 1 \mathrm{H}), 1.44-1.24(\mathrm{~m}, 3 \mathrm{H}), 1.22-1.13(\mathrm{~m}, 1 \mathrm{H}) .{ }^{13} \mathrm{C}$ NMR $\left(126 \mathrm{MHz} \mathrm{CDCl}_{3}\right) \delta 195.12,165.25,134.68,127.82,127.47,126.62,74.43,44.64,24.98$, 22.94, 14.69, 14.01. HRMS ESI [M-H] $]^{-}$calculated for $\mathrm{C}_{12} \mathrm{H}_{12} \mathrm{NO}_{4} \mathrm{~S}_{2} 298.0213$, found 298.0208. 
<smiles>CN1C(=O)[C@@H](c2ccc(Cl)nc2)[C@@H](C(=O)C2CC2)S1(=O)=O</smiles>

( \pm )-(4R,5R)-4-(6-Chloropyridin-3-yl)-5-(cyclopropanecarbonyl)-2-methylisothiazolidin3-one 1,1-dioxide (5o). Following the general procedure, the title compound (16.4 mg) was obtained in $50 \%$ yield. ${ }^{1} \mathrm{H} \mathrm{NMR}\left(500 \mathrm{MHz}, \mathrm{CDCl}_{3}\right) \delta 8.33(\mathrm{~d}, J=2.5 \mathrm{~Hz}, 1 \mathrm{H}), 7.60(\mathrm{dd}, J=$ 8.3, $2.6 \mathrm{~Hz}, 1 \mathrm{H}), 7.35(\mathrm{~d}, J=8.3 \mathrm{~Hz}, 1 \mathrm{H}), 4.89(\mathrm{~d}, J=8.5 \mathrm{~Hz}, 1 \mathrm{H}), 4.83(\mathrm{~d}, J=8.5 \mathrm{~Hz}, 1 \mathrm{H})$, $3.18(\mathrm{~s}, 3 \mathrm{H}), 2.26(\mathrm{tt}, J=7.4,4.6 \mathrm{~Hz}, 1 \mathrm{H}), 1.41-1.35(\mathrm{~m}, 1 \mathrm{H}), 1.32-1.26(\mathrm{~m}, 2 \mathrm{H}), 1.21-$ $1.13(\mathrm{~m}, 1 \mathrm{H}) .{ }^{13} \mathrm{C} \mathrm{NMR}\left(126 \mathrm{MHz}, \mathrm{CDCl}_{3}\right) \delta 194.85,165.27,152.15,150.11,139.06,128.73$, 124.96, 74.07, 46.33, 25.11, 22.82, 15.17, 14.35. HRMS ESI [M-H] ${ }^{-}$calculated for $\mathrm{C}_{13} \mathrm{H}_{12} \mathrm{ClN}_{2} \mathrm{O}_{4} \mathrm{~S} 327.0212$, found 327.0218.<smiles>C=CCCCN1C(=O)[C@H](c2ccc(C)cc2)[C@@H](C(=O)C2CC2)S1(=O)=O</smiles>

( \pm )-(4R,5R)--5-(Cyclopropanecarbonyl)-2-(pent-4-en-1-yl)-4-( $p$-tolyl)isothiazolidin-3-one 1,1-dioxide (5p). Following the general procedure, the title compound (16.6 mg) was obtained in $46 \%$ yield. ${ }^{1} \mathrm{H}$ NMR $\left(400 \mathrm{MHz}, \mathrm{CDCl}_{3}\right) \delta 7.19(\mathrm{~d}, J=8.2 \mathrm{~Hz}, 2 \mathrm{H}), 7.15(\mathrm{~d}, J=8.5 \mathrm{~Hz}, 2 \mathrm{H})$, 5.80 (ddt, $J=16.9,10.2,6.6 \mathrm{~Hz}, 1 \mathrm{H}), 5.08$ (dq, $J=17.1,1.6 \mathrm{~Hz}, 1 \mathrm{H}), 5.02$ (dq, $J=10.2,1.3$ $\mathrm{Hz}, 1 \mathrm{H}), 4.85(\mathrm{~d}, J=7.8 \mathrm{~Hz}, 1 \mathrm{H}), 4.81(\mathrm{~d}, J=7.8 \mathrm{~Hz}, 1 \mathrm{H}), 3.66(\mathrm{dd}, J=8.4,6.7 \mathrm{~Hz}, 2 \mathrm{H}), 2.34$ $(\mathrm{s}, 3 \mathrm{H}), 2.26-2.18(\mathrm{~m}, 1 \mathrm{H}), 2.17-2.10(\mathrm{~m}, 2 \mathrm{H}), 1.92-1.81(\mathrm{~m}, 2 \mathrm{H}), 1.37-1.30(\mathrm{~m}, 1 \mathrm{H})$, $1.29-1.21(\mathrm{~m}, 2 \mathrm{H}), 1.14-1.08(\mathrm{~m}, 1 \mathrm{H}) .{ }^{13} \mathrm{C} \mathrm{NMR}\left(126 \mathrm{MHz}, \mathrm{CDCl}_{3}\right) \delta 195.52,166.75$, $138.79,136.89,131.24,130.17,128.54,115.97,74.83,49.08,40.32,30.86,27.50,22.88,21.27$, 14.62, 13.76. HRMS ESI [M-H] $]^{-}$calculated for $\mathrm{C}_{19} \mathrm{H}_{22} \mathrm{NO}_{4} \mathrm{~S} 360.1275$, found 360.1267.<smiles>Cc1ccc([C@H]2C(=O)N(C3CCCCC3)S(=O)(=O)[C@H]2C(=O)C2CC2)cc1</smiles>

( \pm )-(4R,5R)-2-Cyclohexyl-5-(cyclopropanecarbonyl)-4-(p-tolyl)isothiazolidin-3-one 1,1dioxide (5q). Following the general procedure, the title compound $(18.1 \mathrm{mg})$ was obtained in $48 \%$ yield. ${ }^{1} \mathrm{H}$ NMR $\left(400 \mathrm{MHz}, \mathrm{CDCl}_{3}\right) \delta 7.18(\mathrm{~d}, J=8.0 \mathrm{~Hz}, 2 \mathrm{H}), 7.14(\mathrm{~d}, J=8.0 \mathrm{~Hz}, 2 \mathrm{H})$, $4.80(\mathrm{~d}, J=7.7 \mathrm{~Hz}, 1 \mathrm{H}), 4.74(\mathrm{~d}, J=7.7 \mathrm{~Hz}, 1 \mathrm{H}), 4.05$ (tt, $J=12.2,4.1 \mathrm{~Hz}, 1 \mathrm{H}), 2.34(\mathrm{~s}, 3 \mathrm{H})$, $2.24-2.16(\mathrm{~m}, 1 \mathrm{H}), 2.12-2.04(\mathrm{~m}, 2 \mathrm{H}), 2.02-1.94(\mathrm{~m}, 2 \mathrm{H}), 1.91-1.82(\mathrm{~m}, 2 \mathrm{H}), 1.70-1.64$ $(\mathrm{m}, 1 \mathrm{H}), 1.37-1.28(\mathrm{~m}, 3 \mathrm{H}), 1.27-1.18(\mathrm{~m}, 3 \mathrm{H}), 1.14-1.06(\mathrm{~m}, 1 \mathrm{H}) .{ }^{13} \mathrm{C} \mathrm{NMR}(126 \mathrm{MHz}$, $\left.\mathrm{CDCl}_{3}\right) \delta 195.77,166.56,138.65,131.67,130.12,128.51,74.90,56.23,48.68,30.56,30.07$, 
26.19, 26.12, 25.07, 22.86, 21.27, 14.51, 13.68. HRMS ESI [M-H] $]^{-}$calculated for $\mathrm{C}_{20} \mathrm{H}_{24} \mathrm{NO}_{4} \mathrm{~S}$ 374.1432 , found 374.1424 .

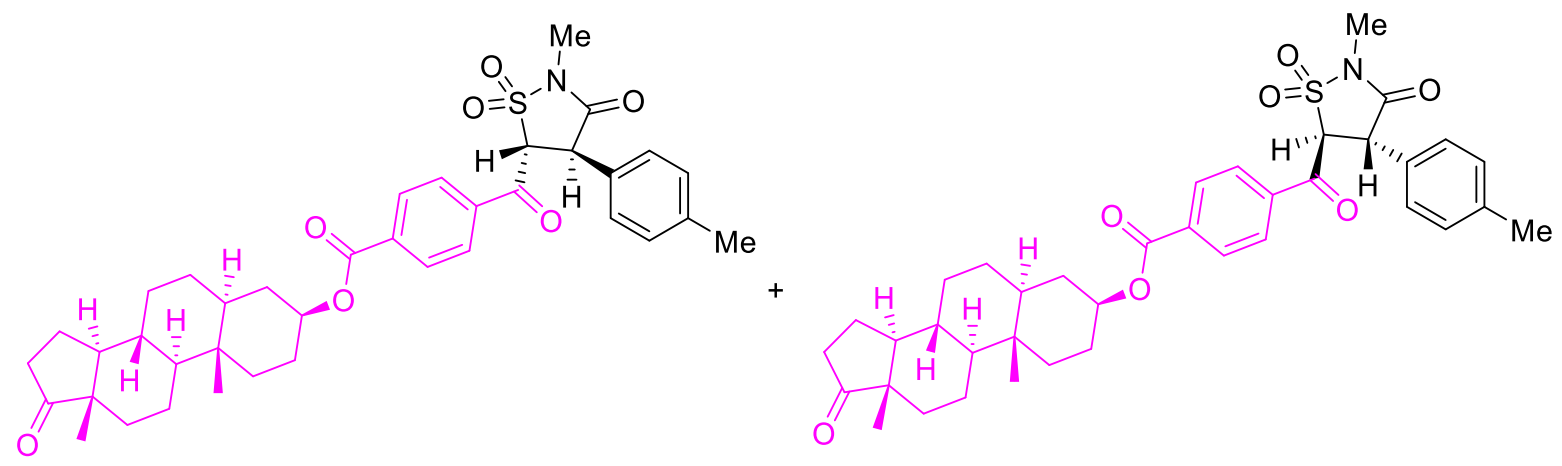

from Epiandrosterone

$(3 S, 5 S, 8 R, 9 S, 10 S, 13 S, 14 S)-10,13-D i m e t h y l-17-o x o h e x a d e c a h y d r o-1 H$ cyclopenta[ $a]$ phenanthren-3-yl 4-(2-methyl-1,1-dioxido-3-oxo-4-(p-tolyl)isothiazolidine5-carbonyl)benzoate (6a). Following the general procedure, the product $(36.3 \mathrm{mg})$ was obtained in 55\% yield as an inseparable mixture of 2 diastereomers (1:1, estimated by HPLC; signals of the 2 isomers cannot be distinguished by $\left.{ }^{1} \mathrm{H} \mathrm{NMR}\right) .{ }^{1} \mathrm{H} \mathrm{NMR}\left(500 \mathrm{MHz}, \mathrm{CDCl}_{3}\right) \delta$ $8.18(\mathrm{~d}, J=9.0 \mathrm{~Hz}, 2 \mathrm{H}), 8.15(\mathrm{~d}, J=8.8 \mathrm{~Hz}, 2 \mathrm{H}), 7.22(\mathrm{~d}, J=8.2 \mathrm{~Hz}, 2 \mathrm{H}), 7.18(\mathrm{~d}, J=8.0 \mathrm{~Hz}$, $2 \mathrm{H}), 5.45(\mathrm{~d}, J=7.8 \mathrm{~Hz}, 1 \mathrm{H}), 5.17(\mathrm{~d}, J=7.7 \mathrm{~Hz}, 1 \mathrm{H}), 4.96(\mathrm{tt}, J=10.8,4.9 \mathrm{~Hz}, 1 \mathrm{H}), 3.19$ (s, $3 \mathrm{H}), 2.44$ (dd, $J=19.2,8.9 \mathrm{~Hz}, 1 \mathrm{H}), 2.32(\mathrm{~s}, 3 \mathrm{H}), 2.08$ (dt, $J=19.4,9.0 \mathrm{~Hz}, 1 \mathrm{H}), 2.00-1.90$ $(\mathrm{m}, 2 \mathrm{H}), 1.82(\mathrm{t}, J=3.4 \mathrm{~Hz}, 1 \mathrm{H}), 1.80(\mathrm{t}, J=3.5 \mathrm{~Hz}, 1 \mathrm{H}), 1.78-1.72(\mathrm{~m}, 1 \mathrm{H}), 1.71-1.64(\mathrm{~m}$, 2H), $1.61-1.47(\mathrm{~m}, 3 \mathrm{H}), 1.46-1.39(\mathrm{~m}, 1 \mathrm{H}), 1.38-1.33(\mathrm{~m}, 2 \mathrm{H}), 1.32-1.21(\mathrm{~m}, 4 \mathrm{H}), 1.16$ $-1.08(\mathrm{~m}, 1 \mathrm{H}), 1.05-0.95(\mathrm{~m}, 1 \mathrm{H}), 0.90(\mathrm{~s}, 3 \mathrm{H}), 0.87(\mathrm{~s}, 3 \mathrm{H}), 0.80-0.71(\mathrm{~m}, 1 \mathrm{H}) .{ }^{13} \mathrm{C}$ NMR $\left(126 \mathrm{MHz}, \mathrm{CDCl}_{3}\right) \delta 221.31,184.89,166.60,164.79,139.08,138.09,136.63,131.02,130.39$, $130.30,129.39,128.60,75.25,70.32,54.47,51.52,50.11,47.93,44.85,36.85,35.99,35.85$, $35.20,34.09,31.68,30.96,28.43,27.59,25.14,21.93,21.27,20.65,13.97,12.43$. HRMS ESI $[\mathrm{M}-\mathrm{H}]^{-}$calculated for $\mathrm{C}_{38} \mathrm{H}_{44} \mathrm{NO}_{7} \mathrm{~S} 658.2844$, found 658.2852 .

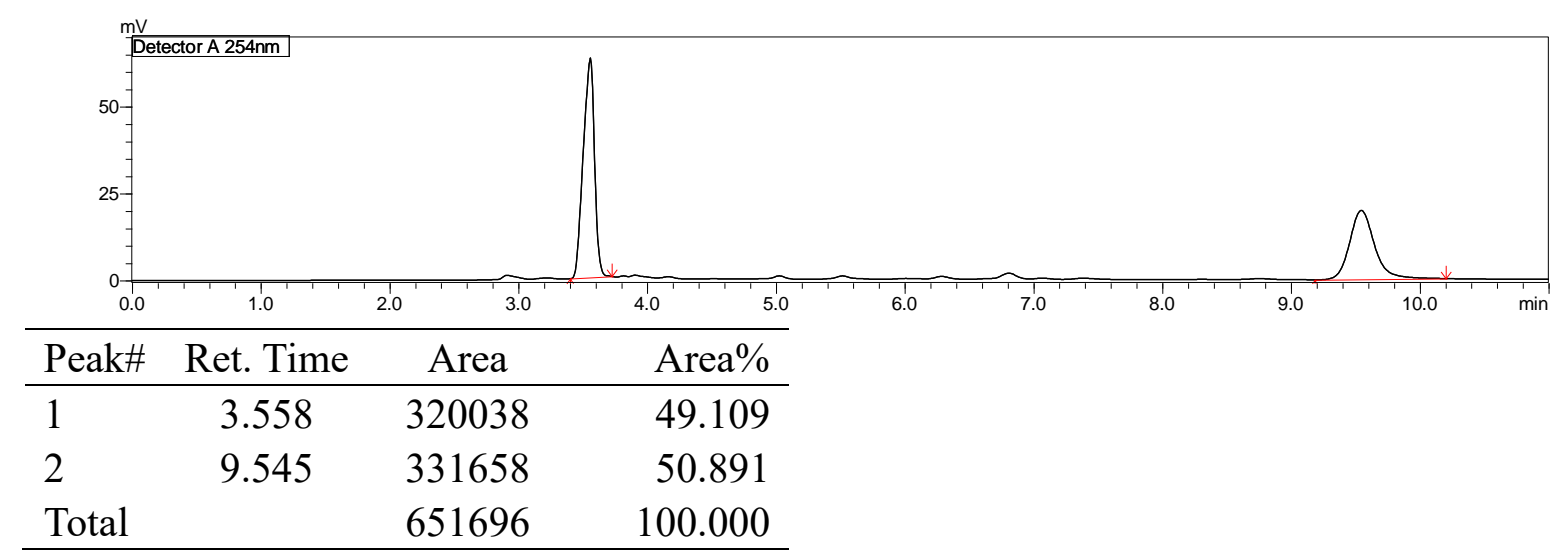




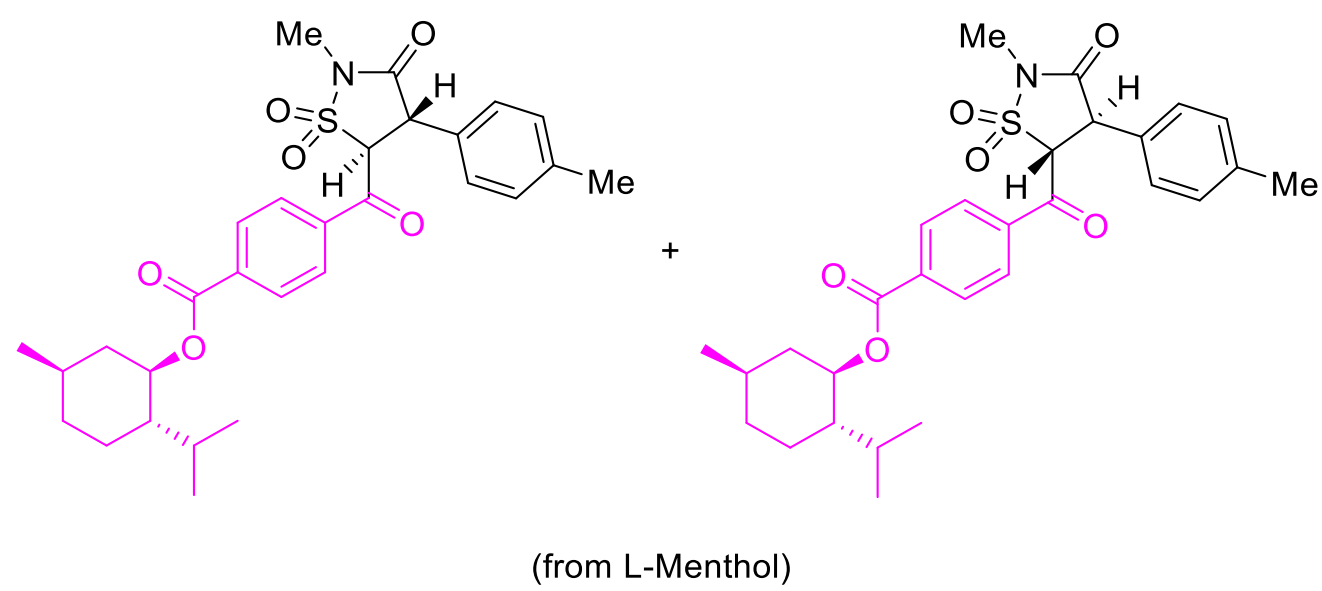

$(1 R, 2 S, 5 R)$-2-isopropyl-5-methylcyclohexyl $\quad 4-(4 S, 5 S)$-(2-methyl-1,1-dioxido-3-oxo-4-( $p$ tolyl)isothiazolidine-5-carbonyl)benzoate $+(1 R, 2 S, 5 R)$-2-isopropyl-5-methylcyclohexyl 4-(4R,5R)-(2-methyl-1,1-dioxido-3-oxo-4-( $p$-tolyl)isothiazolidine-5-carbonyl)benzoate

(6b). Following the general procedure, the product $(31.5 \mathrm{mg})$ was obtained in $60 \%$ yield as an inseparable mixture of 2 diastereomers (1:1, estimated by ${ }^{1} \mathrm{H}$ NMR). ${ }^{1} \mathrm{H}$ NMR $(400 \mathrm{MHz}$, $\left.\mathrm{CDCl}_{3}\right) \delta 8.23-8.12(\mathrm{~m}, 4 \mathrm{H}), 7.24-7.16(\mathrm{~m}, 4 \mathrm{H}), 5.46(\mathrm{~d}, J=7.7 \mathrm{~Hz}, 1 \mathrm{H}), 5.17(\mathrm{~d}, J=7.7$ $\mathrm{Hz}, 1 \mathrm{H}), 4.96$ (td, $J=10.9,1.7 \mathrm{~Hz}, 0.5 \mathrm{H}), 4.95$ (td, $J=10.9,1.7 \mathrm{~Hz}, 0.5 \mathrm{H}), 3.19(\mathrm{~s}, 3 \mathrm{H}), 2.32$ (s, 3H), $2.14-2.08(\mathrm{~m}, 1 \mathrm{H}), 1.97-1.86(\mathrm{~m}, 1 \mathrm{H}), 1.79-1.70(\mathrm{~m}, 2 \mathrm{H}), 1.65-1.50(\mathrm{~m}, 2 \mathrm{H})$, $1.18-1.06(\mathrm{~m}, 2 \mathrm{H}), 0.94(\mathrm{~d}, J=5.7 \mathrm{~Hz}, 3 \mathrm{H}), 0.92(\mathrm{~d}, J=6.0 \mathrm{~Hz}, 1.5 \mathrm{H}), 0.91(\mathrm{~d}, J=6.8 \mathrm{~Hz}$, $1.5 \mathrm{H}), 0.78(\mathrm{~d}, J=6.9 \mathrm{~Hz}, 3 \mathrm{H}) .{ }^{13} \mathrm{C} \mathrm{NMR}\left(126 \mathrm{MHz}, \mathrm{CDCl}_{3}\right) \delta 184.89,166.60,164.79,139.06$, $138.08,138.07,136.60,131.00,130.41,130.29,129.42,129.40,128.58,128.57,76.03,76.02$, $70.30,70.28,50.12,50.10,47.36,47.35,40.99,40.98,34.36,31.60,26.74,26.64,25.14,23.79$, 23.69, 22.15, 21.26, 20.90, 20.84, 16.67, 16.57. HRMS ESI [M-H] ${ }^{-}$calculated for $\mathrm{C}_{29} \mathrm{H}_{34} \mathrm{NO}_{6} \mathrm{~S}$ 524.2112, found 524.2105.

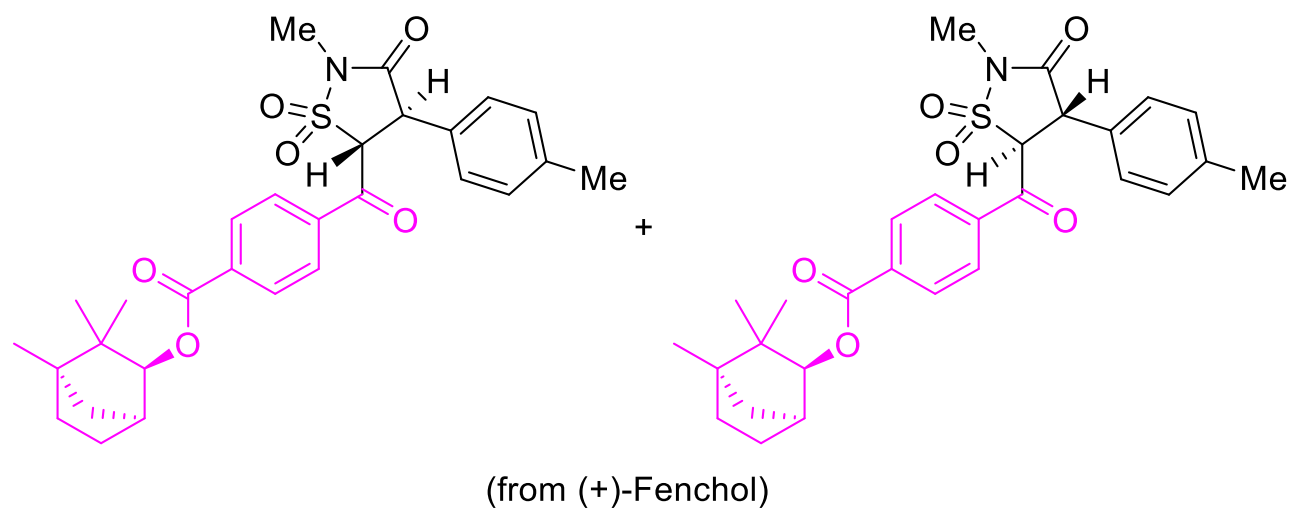

$(1 S, 2 S, 4 R)-3,3,4-t r i m e t h y l b i c y c l o[2.2 .1]$ heptan-2-yl $\quad 4-((4 S, 5 S)$-2-methyl-1,1-dioxido-3oxo-4-( $p$-tolyl)isothiazolidine-5-carbonyl)benzoate $\quad+\quad(1 S, 2 S, 4 R)-3,3,4-$ trimethylbicyclo[2.2.1]heptan-2-yl 4-((4R,5R)-2-methyl-1,1-dioxido-3-oxo-4-(ptolyl)isothiazolidine-5-carbonyl)benzoate (6c). Following the general procedure, the product $(31.9 \mathrm{mg})$ was obtained in $61 \%$ yield as an inseparable mixture of 2 diastereomers $(1: 1$, estimated by ${ }^{1} \mathrm{H}$ NMR). ${ }^{1} \mathrm{H}$ NMR $\left(400 \mathrm{MHz}, \mathrm{CDCl}_{3}\right) \delta 8.21(\mathrm{~d}, J=8.9 \mathrm{~Hz}, 2 \mathrm{H}), 8.18(\mathrm{~d}, J=$ $8.7 \mathrm{~Hz}, 2 \mathrm{H}), 7.22(\mathrm{~d}, J=8.3 \mathrm{~Hz}, 2 \mathrm{H}), 7.18(\mathrm{~d}, J=8.2 \mathrm{~Hz}, 2 \mathrm{H}), 5.47(\mathrm{~d}, J=7.7 \mathrm{~Hz}, 1 \mathrm{H}), 5.17$ $(\mathrm{d}, J=7.7 \mathrm{~Hz}, 1 \mathrm{H}), 4.64(\mathrm{t}, J=1.7 \mathrm{~Hz}, 1 \mathrm{H}), 3.20(\mathrm{~s}, 3 \mathrm{H}), 2.32(\mathrm{~s}, 3 \mathrm{H}), 1.94-1.84(\mathrm{~m}, 1 \mathrm{H})$, $1.83-1.73(\mathrm{~m}, 2 \mathrm{H}), 1.71-1.62(\mathrm{~m}, 1 \mathrm{H}), 1.59-1.48(\mathrm{~m}, 1 \mathrm{H}), 1.30-1.22(\mathrm{~m}, 2 \mathrm{H}), 1.18(\mathrm{~s}$, 
$3 \mathrm{H}), 1.11(\mathrm{~s}, 1.5 \mathrm{H}), 1.10(\mathrm{~s}, 1.5 \mathrm{H}), 0.83(\mathrm{~s}, 1.5 \mathrm{H}), 0.82(\mathrm{~s}, 1.5 \mathrm{H}) .{ }^{13} \mathrm{C} \mathrm{NMR}\left(126 \mathrm{MHz}, \mathrm{CDCl}_{3}\right)$ $\delta 184.86,166.59,165.58,139.06,138.14,136.41,130.98,130.35,130.29,129.50,128.55$, $87.80,87.78,70.28,50.12$, 48.79, 48.78, 48.50, 41.57, 40.03, 29.87, 26.99, 26.00, 25.13, 21.25, 20.42, 20.40, 19.60. HRMS ESI [M-H] $]^{-}$calculated for $\mathrm{C}_{29} \mathrm{H}_{32} \mathrm{NO}_{6} \mathrm{~S} 522.1956$, found 522.1947.
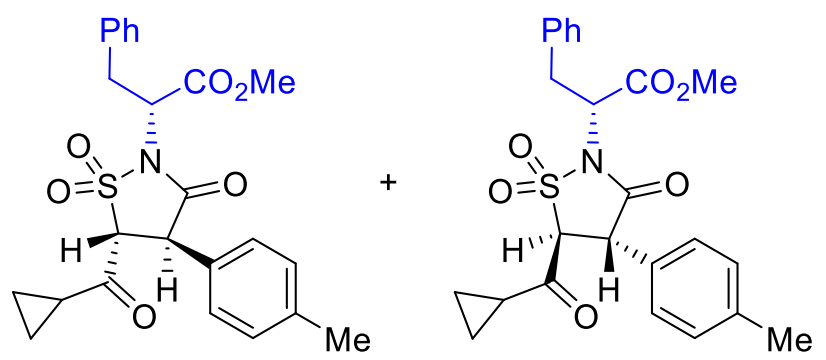

Methyl

$(R)-2-((4 R, 5 R)-5-($ cyclopropanecarbonyl)-1,1-dioxido-3-oxo-4- $(p-$ tolyl)isothiazolidin-2-yl)-3-phenylpropanoate $+\quad$ methyl $\quad(R)-2-((4 S, 5 S)-5-$ (cyclopropanecarbonyl)-1,1-dioxido-3-oxo-4-(p-tolyl)isothiazolidin-2-yl)-3-

phenylpropanoate (6d). Following the general procedure, the product $(27.1 \mathrm{mg})$ was obtained in $59 \%$ yield as an inseparable mixture of 2 diastereomers (1:1, estimated by ${ }^{1} \mathrm{H}$ NMR). ${ }^{1} \mathrm{H}$ NMR (400 MHz, $\left.\mathrm{CDCl}_{3}\right) \delta 7.39-7.23(\mathrm{~m}, 5 \mathrm{H}), 7.14(\mathrm{dd}, J=11.1,7.9 \mathrm{~Hz}, 2 \mathrm{H}), 7.01(\mathrm{dd}, J=$ $12.1,8.1 \mathrm{~Hz}, 2 \mathrm{H}), 4.94-4.87(\mathrm{~m}, 1 \mathrm{H}), 4.79-4.75(\mathrm{~m}, 1 \mathrm{H}), 4.73(\mathrm{~d}, J=8.1 \mathrm{~Hz}, 0.5 \mathrm{H}), 4.69$ $(\mathrm{d}, J=9.0 \mathrm{~Hz}, 0.5 \mathrm{H}), 3.84(\mathrm{~s}, 3 \mathrm{H}), 3.62(\mathrm{dd}, J=14.7,5.6 \mathrm{~Hz}, 0.5 \mathrm{H}), 3.56-3.46(\mathrm{~m}, 1.5 \mathrm{H})$, $2.34(\mathrm{~s}, 1.5 \mathrm{H}), 2.32(\mathrm{~s}, 1.5 \mathrm{H}), 2.18-2.08(\mathrm{~m}, 1 \mathrm{H}), 1.29-1.24(\mathrm{~m}, 1 \mathrm{H}), 1.22-1.13(\mathrm{~m}, 2 \mathrm{H})$, $1.09-1.00(\mathrm{~m}, 1 \mathrm{H}) .{ }^{13} \mathrm{C}$ NMR $\left(126 \mathrm{MHz}, \mathrm{CDCl}_{3}\right) \delta 194.97,194.71,168.23,168.08,167.55$, 167.42 , 138.75, 138.64, 136.20, 136.14, 131.11, 130.75, 130.03, 130.00, 129.48, 129.47, $128.85,128.84,128.79,128.70,127.37,127.29,75.84,75.42,57.07,56.37,53.42,53.34,48.75$, $48.70,34.78,34.40,22.47,21.28,21.26,14.66,14.62,13.54,13.49$. HRMS ESI [M-H] ${ }^{-}$ calculated for $\mathrm{C}_{24} \mathrm{H}_{24} \mathrm{NO}_{6} \mathrm{~S} 454.1330$, found 454.1334 .<smiles>CC(=O)C(C)N1C(=O)[C@H](c2ccc(C)cc2)[C@@H](C(=O)C2CC2)S1(=O)=O</smiles>

Methyl<smiles>CC(=O)C(C)N1C(=O)[C@H](c2ccc(C)cc2)[C@@](C(=O)O)(C(=O)C2CC2)S1(=O)=O</smiles>

$(R)-2-((4 R, 5 R)-5-($ cyclopropanecarbonyl)-1,1-dioxido-3-oxo-4- $(p$ tolyl)isothiazolidin-2-yl)propanoate + methyl $(R)-2-((4 S, 5 S)-5-($ cyclopropanecarbonyl)1,1-dioxido-3-oxo-4-( $p$-tolyl)isothiazolidin-2-yl)propanoate (6e). Following the general procedure, the product $(26.2 \mathrm{mg})$ was obtained in $69 \%$ yield as an inseparable mixture of 2 diastereomers (1:1, estimated by ${ }^{1} \mathrm{H}$ NMR). ${ }^{1} \mathrm{H}$ NMR $\left(400 \mathrm{MHz}, \mathrm{CDCl}_{3}\right) \delta 7.22-7.16(\mathrm{~m}, 4 \mathrm{H})$, $4.97-4.91(\mathrm{~m}, 1 \mathrm{H}), 4.90-4.85(\mathrm{~m}, 1 \mathrm{H}), 4.84(\mathrm{~d}, J=7.7 \mathrm{~Hz}, 0.5 \mathrm{H}), 4.64(\mathrm{q}, J=7.3 \mathrm{~Hz}, 0.5 \mathrm{H})$, $3.82(\mathrm{~s}, 1.5 \mathrm{H}), 3.79(\mathrm{~s}, 1.5 \mathrm{H}), 2.33(\mathrm{~s}, 3 \mathrm{H}), 2.27-2.14(\mathrm{~m}, 1 \mathrm{H}), 1.80(\mathrm{~d}, J=7.6 \mathrm{~Hz}, 1.5 \mathrm{H})$, $1.75(\mathrm{~d}, J=7.4 \mathrm{~Hz}, 1.5 \mathrm{H}), 1.39-1.28(\mathrm{~m}, 1 \mathrm{H}), 1.28-1.20(\mathrm{~m}, 2 \mathrm{H}), 1.14-1.07(\mathrm{~m}, 1 \mathrm{H}) .{ }^{13} \mathrm{C}$ NMR $\left(126 \mathrm{MHz}, \mathrm{CDCl}_{3}\right) \delta 195.27,194.87,169.24,168.70,167.32,166.88,138.79,138.77$, 131.26, 131.08, 130.14, 130.13, 128.87, 128.74, 76.17, 75.66, 53.32, 53.23, 51.15, 50.60, 48.87, $48.83,22.79,22.67,21.28,21.27,15.16,14.66,14.62,14.26,13.72,13.50$. HRMS ESI [M-H] ${ }^{-}$ calculated for $\mathrm{C}_{18} \mathrm{H}_{20} \mathrm{NO}_{6} \mathrm{~S} 378.1017$, found 378.1011. 

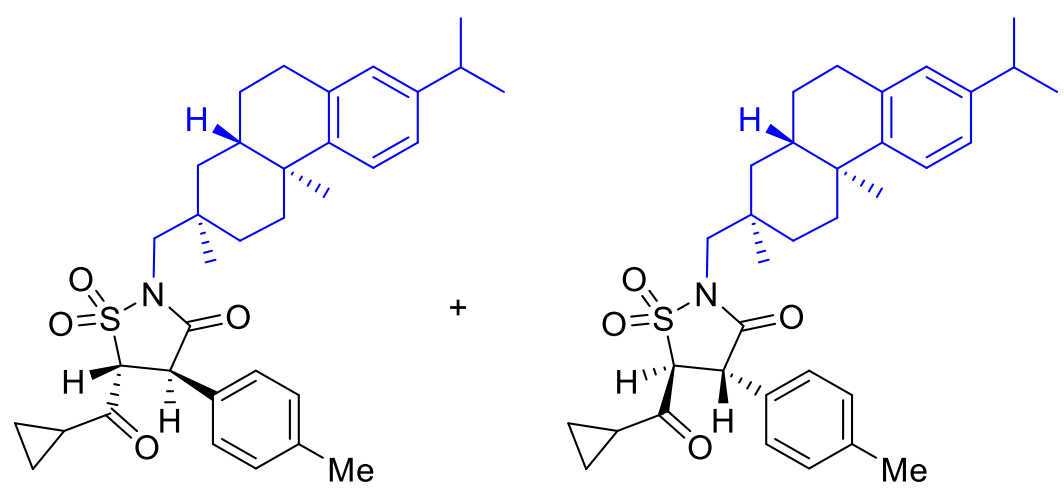

$(4 R, 5 R)-5$-(cyclopropanecarbonyl)-2-(( $(2 R, 4 \mathrm{a} S, 10 \mathrm{a} S)-7$-isopropyl-2,4a-dimethyl-

1,2,3,4,4a,9,10,10a-octahydrophenanthren-2-yl)methyl)-4-(p-tolyl)isothiazolidin-3-one 1,1-dioxide $+(4 S, 5 S)-5$-(cyclopropanecarbonyl)-2-(( $2 R, 4 a S, 10 a S)$-7-isopropyl-2,4adimethyl-1,2,3,4,4a,9,10,10a-octahydrophenanthren-2-yl)methyl)-4-( $p$ -

tolyl)isothiazolidin-3-one 1,1-dioxide (6f). Following the general procedure, the product $(18.0 \mathrm{mg})$ was obtained in $32 \%$ yield as an inseparable mixture of 2 diastereomers $(1: 1$, estimated by ${ }^{1} \mathrm{H}$ NMR). ${ }^{1} \mathrm{H}$ NMR $\left(500 \mathrm{MHz}, \mathrm{CDCl}_{3}\right) \delta 7.20-7.11(\mathrm{~m}, 5 \mathrm{H}), 6.98(\mathrm{ddd}, J=8.1$, $4.3,2.0 \mathrm{~Hz}, 1 \mathrm{H}), 6.88(\mathrm{~d}, J=2.0 \mathrm{~Hz}, 0.5 \mathrm{H}), 6.87(\mathrm{~d}, J=2.0 \mathrm{~Hz}, 0.5 \mathrm{H}), 4.85-4.80(\mathrm{~m}, 2 \mathrm{H})$, $3.70(\mathrm{~d}, J=8.6 \mathrm{~Hz}, 0.46 \mathrm{H}), 3.68(\mathrm{~d}, J=8.7 \mathrm{~Hz}, 0.5 \mathrm{H}), 3.53(\mathrm{~d}, J=14.5 \mathrm{~Hz}, 1 \mathrm{H}), 2.96-2.87$ $(\mathrm{m}, 2 \mathrm{H}), 2.85-2.78(\mathrm{~m}, 1 \mathrm{H}), 2.33(\mathrm{~s}, 0.5 * 3 \mathrm{H}), 2.32(\mathrm{~s}, 0.5 * 3 \mathrm{H}), 2.28(\mathrm{~d}, J=12.7 \mathrm{~Hz}, 1 \mathrm{H})$, $2.24-2.20(\mathrm{~m}, 1 \mathrm{H}), 2.03-1.93(\mathrm{~m}, 1 \mathrm{H}), 1.82-1.76(\mathrm{~m}, 1 \mathrm{H}), 1.73-1.65(\mathrm{~m}, 2 \mathrm{H}), 1.55-1.51$ $(\mathrm{m}, 2 \mathrm{H}), 1.47-1.37(\mathrm{~m}, 2 \mathrm{H}), 1.32-1.27(\mathrm{~m}, 2 \mathrm{H}), 1.24-1.20(\mathrm{~m}, 10 \mathrm{H}), 1.14-1.07(\mathrm{~m}, 1 \mathrm{H})$, $1.06\left(\mathrm{~s}, 0.5^{*} 3 \mathrm{H}\right), 1.05\left(\mathrm{~s}, 0.5^{*} 3 \mathrm{H}\right) .{ }^{13} \mathrm{C} \mathrm{NMR}\left(126 \mathrm{MHz}, \mathrm{CDCl}_{3}\right) \delta 195.68,195.50,168.88$, $168.78,147.25,147.23,145.74,145.70,138.74,138.70,134.94,134.74,130.99,130.96$, 130.12 (2C), 130.08 (2C), 128.67 (2C), 128.61 (2C), 127.11, 126.98, 124.07, 124.03, 123.91, 123.88, 74.86, 74.77, 53.71, 53.41, 49.10, 48.98, 46.16, 45.55, 38.24, 38.16, 38.15, 38.13, $37.78,37.77,37.08,36.56,33.61,33.59,29.93,29.86,25.83,25.63,24.15,24.15,24.14,24.09$, 22.74, 22.65, 21.28, 21.26, 19.55, 19.55, 18.93, 18.65 (2C), 18.38, 14.67, 14.62, 13.69, 13.60 . HRMS ESI [M-H] $]^{-}$calculated for $\mathrm{C}_{34} \mathrm{H}_{42} \mathrm{NO}_{4} \mathrm{~S} 560.2840$, found 560.2834.

\section{Mechanistic studies}

\section{Study on the origin of the diastereoselectivity}

The origin of diastereoselectivity was further investigated in Scheme S2. At room temperature, the reaction after $48 \mathrm{~h}$ gave a mixture of diastereomers (3a:3a' $=2: 1$ ) with poor selectivity (eq $\mathrm{S} 4$ ). As illustrated in eq S5, another two parallel reactions were performed initially under blue LED irradiation at room temperature for $48 \mathrm{~h}$, then the two reaction mixtures were further heated at $80{ }^{\circ} \mathrm{C}$ for $12 \mathrm{~h}$ under blue LED irradiation or in darkness, respectively. The diatereoselective ratios of the two reactions were both improved to an excellent level (>30:1), regardless of in the presence of light or not. We have attempted to isolate the isomers obtained at room temperature (with 2:1 d.r.). However, the cis-isomer underwent fast epimerization during chromatography even with silica saturated by triethylamine. These experiments 
suggested that the cis-isomer is not stable, which will easily epimerize to the more stable transisomer under heating or slightly acidic conditions.

Scheme S2. Control experiments to understand the diastereoselectivity<smiles>C#CC(=O)N(C)S(=O)(=O)c1ccc(C)cc1</smiles>

1a $(0.3 \mathrm{mmol})$

2a $(0.1 \mathrm{mmol})$<smiles></smiles>

3a

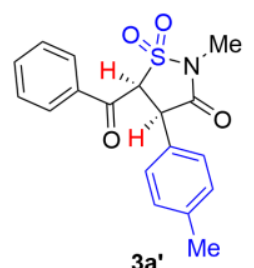

3a' $\mathrm{Me}$

(eq S4)

$37 \%\left(3 a: 3 a^{\prime}=2: 1\right)$<smiles>O=Cc1ccccc1</smiles>

1 a $(0.3 \mathrm{mmol})$<smiles>C#CC(=O)N(C)S(=O)(=O)c1ccc(C)cc1</smiles>

2a $(0.1 \mathrm{mmol})$ $\operatorname{eosin} \mathrm{Y}(4 \mathrm{~mol} \%)$

blue LED, $18 \mathrm{~W}$ $\mathrm{CH}_{3} \mathrm{CN}(0.1 \mathrm{M}), \mathrm{RT}, 48 \mathrm{~h}$

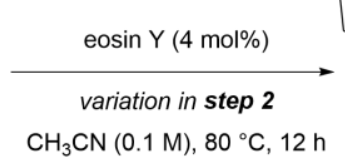

$\mathrm{CH}_{3} \mathrm{CN}(0.1 \mathrm{M}), 80^{\circ} \mathrm{C}, 12 \mathrm{~h}$

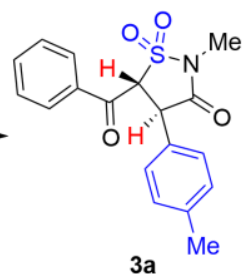

3a

(eq S5)

blue LED, 18 W in step 2: $57 \%$ (3a : 3a' > 30:1) darkness in step $2: 37 \%\left(3 a: 3 a^{\prime}>30: 1\right)$

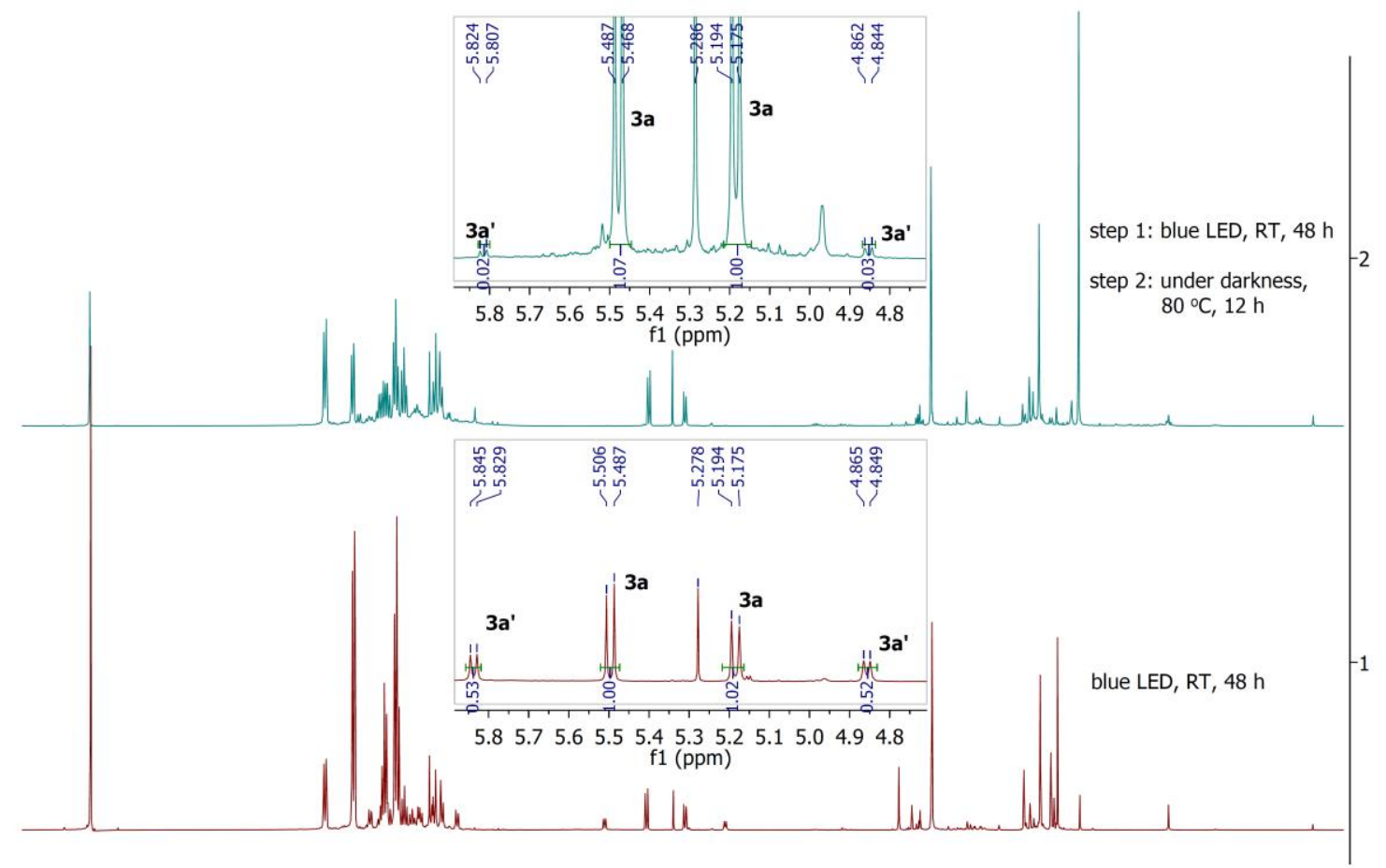

$\begin{array}{llllllllllllllllllllll}0.5 & 10.0 & 9.5 & 9.0 & 8.5 & 8.0 & 7.5 & 7.0 & 6.5 & 6.0 & 5.5 & 5.0 & 4.5 & 4.0 & 3.5 & 3.0 & 2.5 & 2.0 & 1.5 & 1.0 & 0.5 & 0.0\end{array}$ 


\section{Evaluation of different modes of HAT catalysis}

The examination on the effectiveness of different hydrogen atom transfer (HAT)-based photocatalytic systems was shown in Table S1. Neutral eosin Y as a direct HAT photocatalyst promote the current reaction most efficiently (entry 1). The addition of base $\left(\mathrm{Cs}_{2} \mathrm{CO}_{3}\right)$ resulted in decomposition of $\mathbf{2 a}$ (entry 2). Catalytic systems consisting of a photoredox catalyst and a HAT agent were not effective (entries 3-9). Other direct HAT photocatalysts gave lower yields (entries 10-11) or no product (entries 12-13).

\section{Table S1. Control experiment using other HAT catalytic systems}

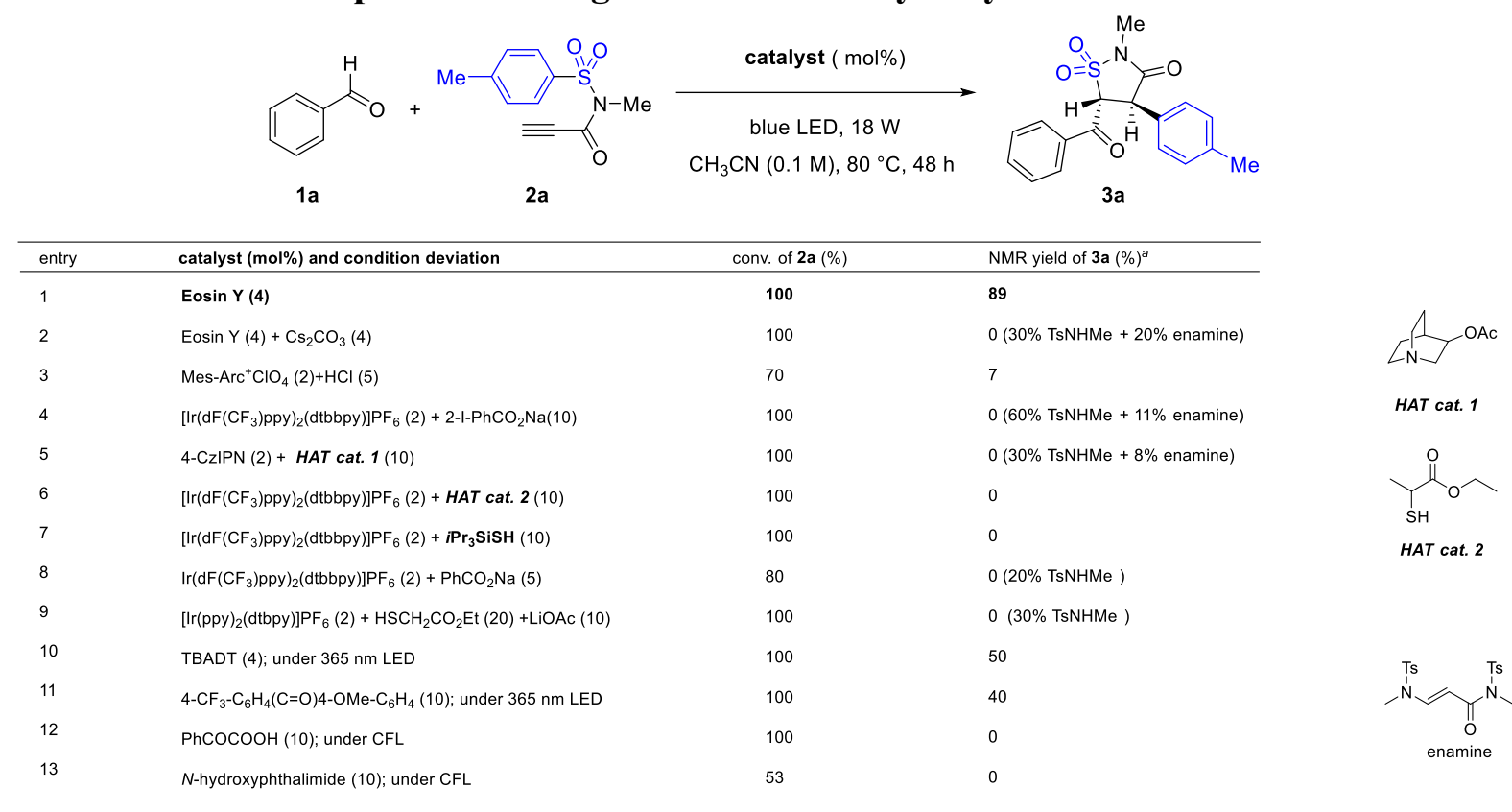

\section{Control experiments indicating the involvement of vinyl radicals}

The involvement of vinyl radical intermediates was suggested by the concurrent formation of two products (5r and 8) using 4-F-benzaldehyde $\mathbf{1 i}$ and $N$-tosyl- $N$-isobutyl propiolamide $2 \mathbf{r}$ (Scheme S3). While the formation of isothiazolidione 5r results from Smiles rearrangement/5endo-trig cyclization of the vinyl radical, production of $\gamma$-lactam $\mathbf{8}$ is rationalized by a competitive pathway of 1,5-HAT/5-exo-dig cyclization. The structure of $\mathbf{8}$ was further elucidated by NOESY measurement (Figure S3). 
Scheme $S 3$. Divergent reaction pathways of vinyl radicals
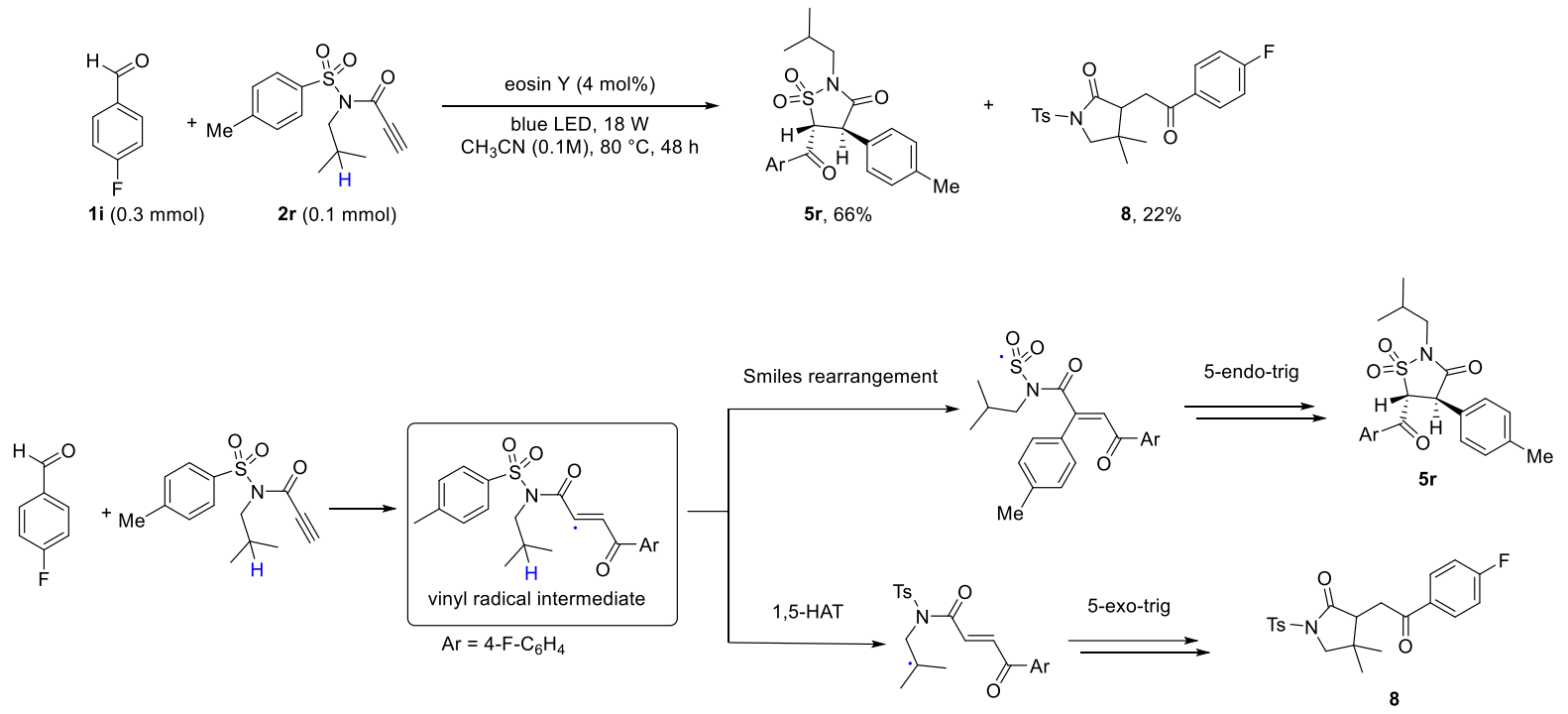<smiles>Cc1ccc([C@H]2C(=O)N(CC(C)C)S(=O)(=O)[C@H]2C(=O)c2ccc(F)cc2)cc1</smiles>

( \pm )-(4R,5R)-5-(4-fluorobenzoyl)-2-isobutyl-4-(p-tolyl)isothiazolidin-3-one 1,1-dioxide (5r). Following the general procedure, the title compound $(26.7 \mathrm{mg})$ was obtained in $66 \%$ yield. ${ }^{1} \mathrm{H}$ NMR (400 MHz, $\left.\mathrm{CDCl}_{3}\right) \delta 8.15(\mathrm{dd}, J=9.0,5.2 \mathrm{~Hz}, 2 \mathrm{H}), 7.24-7.15(\mathrm{~m}, 6 \mathrm{H}), 5.38(\mathrm{~d}, J=7.7$ $\mathrm{Hz}, 1 \mathrm{H}), 5.14(\mathrm{~d}, J=7.6 \mathrm{~Hz}, 1 \mathrm{H}), 3.51(\mathrm{~d}, J=7.7 \mathrm{~Hz}, 2 \mathrm{H}), 2.32(\mathrm{~s}, 3 \mathrm{H}), 2.27-2.16(\mathrm{~m}, 1 \mathrm{H})$, $0.99(\mathrm{~d}, J=6.8 \mathrm{~Hz}, 3 \mathrm{H}), 0.98(\mathrm{~d}, J=6.8 \mathrm{~Hz}, 3 \mathrm{H}) .{ }^{13} \mathrm{C} \mathrm{NMR}\left(126 \mathrm{MHz}, \mathrm{CDCl}_{3}\right) \delta 183.74$, $167.14,167.12\left(\mathrm{~d},{ }^{1} J_{\mathrm{C}-\mathrm{F}}=259.7 \mathrm{~Hz}\right), 138.92,132.47\left(\mathrm{~d},{ }^{3} J_{\mathrm{C}-\mathrm{F}}=10.0 \mathrm{~Hz}, 2 \mathrm{C}\right), 131.91\left(\mathrm{~d},{ }^{4} J_{\mathrm{C}-}\right.$ $\mathrm{F}=2.9 \mathrm{~Hz}), 131.30,130.26(2 \mathrm{C}), 128.57(2 \mathrm{C}), 116.72\left(\mathrm{~d},{ }^{2} J_{\mathrm{C}-\mathrm{F}}=22.2 \mathrm{~Hz}, 2 \mathrm{C}\right), 70.05,49.89$, 48.18, 27.69, 21.26, 20.12, 20.03. ${ }^{19} \mathrm{~F}$ NMR $\left(377 \mathrm{MHz} \mathrm{CDCl}_{3}\right) \delta-100.68$. HRMS ESI [M-H] ${ }^{-}$ calculated for $\mathrm{C}_{21} \mathrm{H}_{21} \mathrm{FNO}_{4} \mathrm{~S} 402.1181$, found 402.1174 .<smiles>CC1(C)CN(S)C(=O)C1CC(=O)c1ccc(F)cc1</smiles>

3-(2-(4-fluorophenyl)-2-oxoethyl)-4,4-dimethyl-1-tosylpyrrolidin-2-one (8). Following the general procedure, the title compound $(8.9 \mathrm{mg})$ was obtained in $22 \%$ yield. ${ }^{1} \mathrm{H}$ NMR $(400 \mathrm{MHz}$, $\left.\mathrm{CDCl}_{3}\right) \delta 7.96(\mathrm{dd}, J=8.9,5.3 \mathrm{~Hz}, 2 \mathrm{H}), 7.90(\mathrm{~d}, J=8.4 \mathrm{~Hz}, 2 \mathrm{H}), 7.33(\mathrm{~d}, J=8.6 \mathrm{~Hz}, 2 \mathrm{H}), 7.12$ (t, $J=8.6 \mathrm{~Hz}, 2 \mathrm{H}), 3.71(\mathrm{~d}, J=9.8 \mathrm{~Hz}, 1 \mathrm{H}), 3.53(\mathrm{~d}, J=9.9 \mathrm{~Hz}, 1 \mathrm{H}), 3.32(\mathrm{dd}, J=17.7,5.7$ $\mathrm{Hz}, 1 \mathrm{H}), 3.16(\mathrm{t}, J=6.0 \mathrm{~Hz}, 1 \mathrm{H}), 2.77(\mathrm{dd}, J=17.7,6.3 \mathrm{~Hz}, 1 \mathrm{H}), 2.44(\mathrm{~s}, 3 \mathrm{H}), 1.14(\mathrm{~s}, 3 \mathrm{H})$, $0.93(\mathrm{~s}, 3 \mathrm{H}) .{ }^{13} \mathrm{C} \mathrm{NMR}\left(126 \mathrm{MHz}, \mathrm{CDCl}_{3}\right) \delta 195.59,174.14,166.06\left(\mathrm{~d},{ }^{1} J_{\mathrm{C}-\mathrm{F}}=255.7 \mathrm{~Hz}\right)$, 145.33, 135.26, $133.02\left(\mathrm{~d},{ }^{4} J_{\mathrm{C}-\mathrm{F}}=3.2 \mathrm{~Hz}\right), 130.96\left(\mathrm{~d},{ }^{3} J_{\mathrm{C}-\mathrm{F}}=9.3 \mathrm{~Hz}, 2 \mathrm{C}\right), 129.87(2 \mathrm{C}), 128.11$ (2C), $115.95\left(\mathrm{~d},{ }^{2} J_{\mathrm{C}-\mathrm{F}}=21.9 \mathrm{~Hz}, 2 \mathrm{C}\right), 58.84,49.60,37.20,33.44,25.47,22.06,21.84 .{ }^{19} \mathrm{~F} \mathrm{NMR}$ 
(377 $\left.\mathrm{MHz}, \mathrm{CDCl}_{3}\right) \delta$-104.56. HRMS ESI $[\mathrm{M}-\mathrm{H}]^{-}$calculated for $\mathrm{C}_{21} \mathrm{H}_{21} \mathrm{FNO}_{4} \mathrm{~S}$ 402.1181, found 402.1172 .

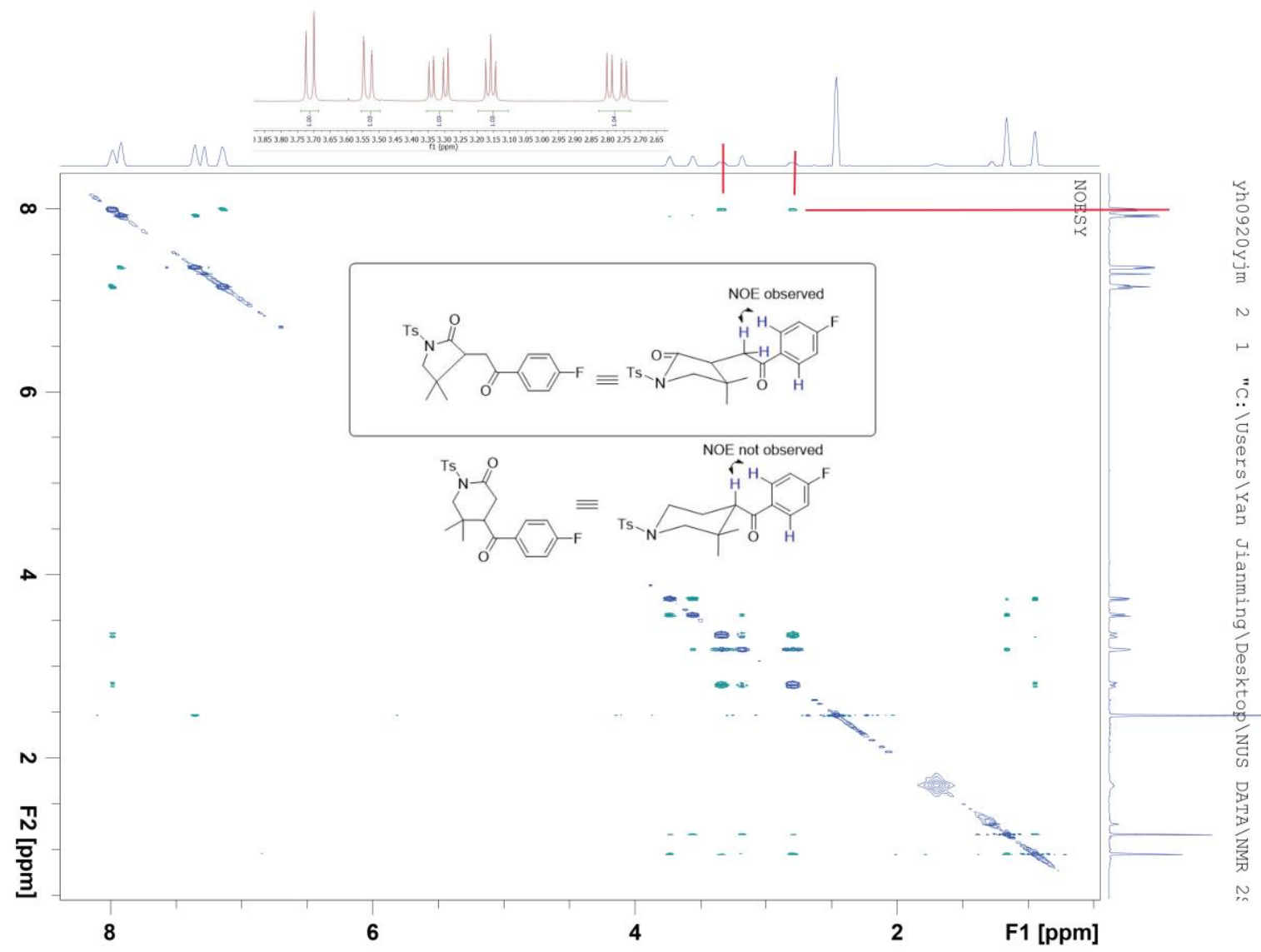

Figure S3. NOSESY spectrum of compound $\mathbf{8}$.

\section{Evidence for the involvement of acyl radicals}

The involvement of acyl radicals was indicated in Scheme S4. The reaction of pivalaldehyde $\mathbf{1 z}$ and $N$-tosyl- $N$-methyl propiolamide $\mathbf{2 a}$ under standard conditions afforded $\mathbf{3 z}$ in which decarbonylation was observed. Facile decarbonylation of pivaloyl radical to give stable tertbutyl radical reasonably accounts for this observation (eq S6). Addition of radical scavenger butylated hydroxytoluene (BHT) to the reaction mixture of 4-F-benzaldehyde $1 \mathbf{i}$ and $N$-tosyl$N$-methyl propiolamide 2a under standard conditions quenched the reaction (eq S7). No desired product was formed and adduct 7 of BHT and 4-fluorobenzoyl radical was detected by HR ESI-MS analysis (Figure S4). ${ }^{7}$ 
Scheme S4. Involvement of acyl radicals
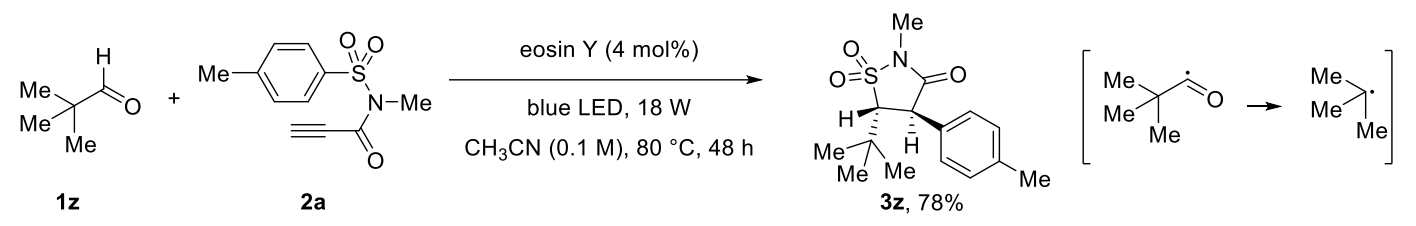

(eq S6)

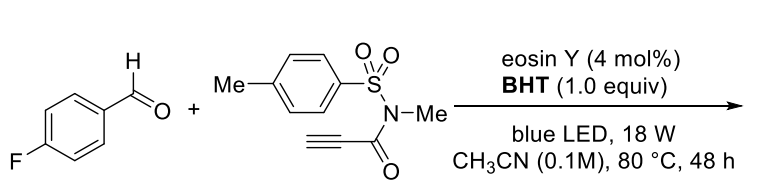

$1 \mathrm{i}$<smiles>[M]C(C)C1[C@H](c2ccc(C)cc2)C(=O)N(C)S1(=O)=O</smiles>

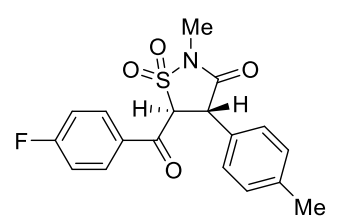

$(0 \%)$

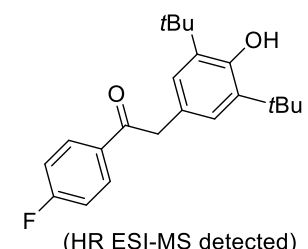

(HR ESI-MS detected) Following the general procedure, the title compound $(23.0 \mathrm{mg})$ was obtained in $78 \%$ yield. ${ }^{1} \mathrm{H}$ NMR (400 MHz, $\left.\mathrm{CDCl}_{3}\right) \delta 7.19(\mathrm{~d}, J=8.1 \mathrm{~Hz}, 2 \mathrm{H}), 7.15(\mathrm{~d}, J=8.3 \mathrm{~Hz}, 2 \mathrm{H}), 4.11(\mathrm{~d}, J=8.4$ $\mathrm{Hz}, 1 \mathrm{H}), 3.73(\mathrm{~d}, J=8.5 \mathrm{~Hz}, 1 \mathrm{H}), 3.11(\mathrm{~s}, 3 \mathrm{H}), 2.34$ (s, 3H), 1.15 (s, 9H). ${ }^{13} \mathrm{C}$ NMR $(126 \mathrm{MHz}$, $\left.\mathrm{CDCl}_{3}\right) \delta 167.64,138.62,133.00,130.24,128.33,74.99,51.10,34.33,27.73,24.40,21.28$. HRMS ESI $[\mathrm{M}+\mathrm{H}]^{+}$calculated for $\mathrm{C}_{15} \mathrm{H}_{20} \mathrm{NO}_{3} \mathrm{~S} 294.1169$, found 294.116.

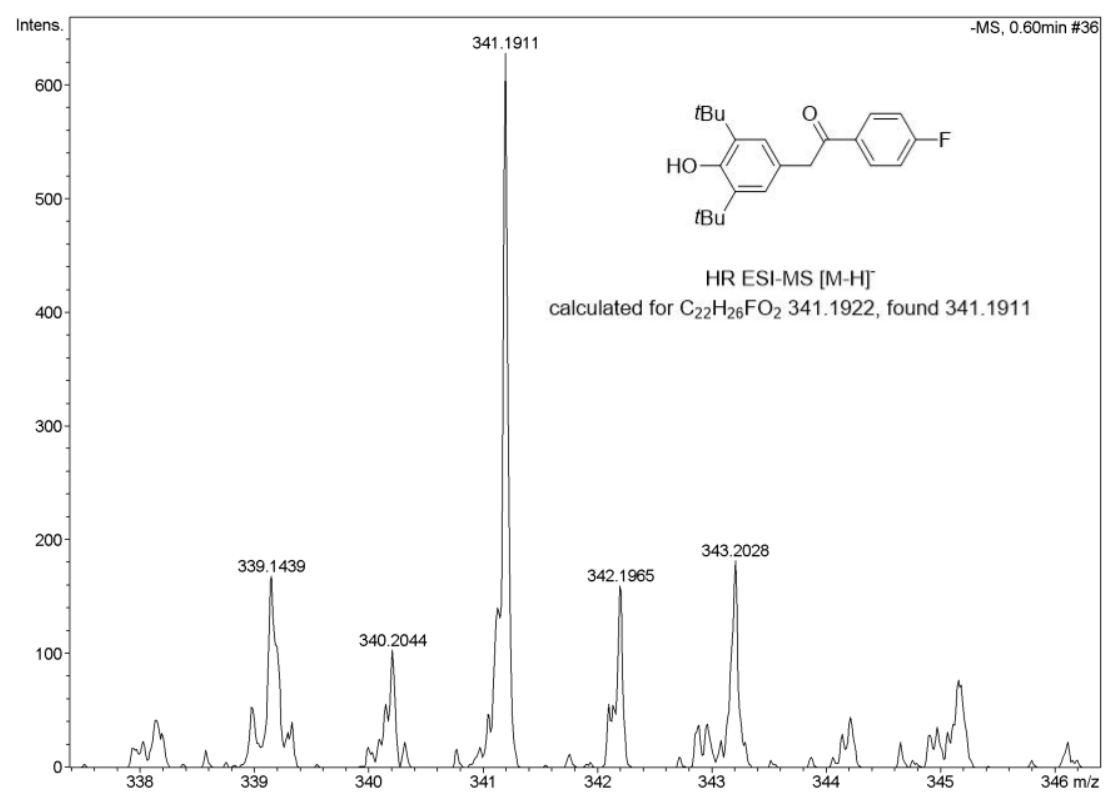

Figure S4. Trapping acyl radicals by BHT. 


\section{Deuterium-labelling study}

Under standard reaction conditions, deuterated 4-fluoro-benzaldehyde $1 \mathbf{i}-d(0.3 \mathrm{mmol}, 37.5 \mathrm{mg})$ and $N$-methyl- $N$-tosyl-propiolamide $2 \mathbf{a}(23.8 \mathrm{mg}, 0.1 \mathrm{mmol})$ reacted to give the corresponding product $3 \mathbf{i}-d$ in $61 \%$ yield. Deuterium incorporation is $42 \%$ at the tertiary benzylic carbon according to ${ }^{1} \mathrm{H}$ NMR analysis (eq S8).<smiles>C#CC(=O)N(C)S(=O)(=O)c1ccc(C=Cc2ccccc2)cc1</smiles>

$1 \mathrm{i}-d(0.3 \mathrm{mmol})$

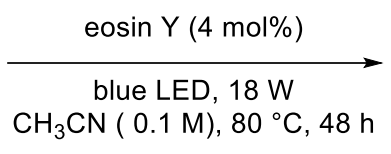
$\mathrm{CH}_{3} \mathrm{CN}(0.1 \mathrm{M}), 80^{\circ} \mathrm{C}, 48 \mathrm{~h}$

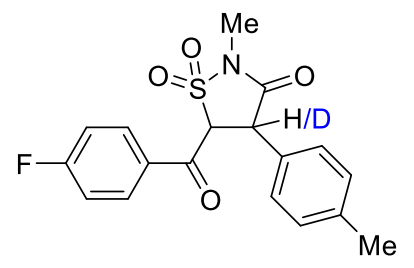

$61 \%$ yield $42 \%$ D incorporation<smiles>Cc1ccc([C@H]2C(=O)N(C)S(=O)(=O)[C@H]2C(=O)c2ccc(F)cc2)cc1</smiles>

( \pm )-5-(4-Fluorobenzoyl)-2-methyl-4-(p-tolyl)isothiazolidin-3-one-4- $\boldsymbol{d}$ 1,1-dioxide. ${ }^{1} \mathrm{H}$ NMR $\left(400 \mathrm{MHz}, \mathrm{CDCl}_{3}\right) \delta 8.19-7.99(\mathrm{~m}, 2 \mathrm{H}), 7.24-7.05(\mathrm{~m}, 6 \mathrm{H}), 5.44-5.29$ (m, 1H), 5.17 (d, J $=7.9 \mathrm{~Hz}, 0.58 \mathrm{H}), 3.19(\mathrm{~s}, 3 \mathrm{H}), 2.32(\mathrm{~s}, 3 \mathrm{H}) .{ }^{19} \mathrm{~F}$ NMR $\left(377 \mathrm{MHz}, \mathrm{CDCl}_{3}\right) \delta-100.51$. HRMS ESI [M-H] $]^{-}$calculated for $\mathrm{C}_{18} \mathrm{H}_{14} \mathrm{DFNO}_{4} \mathrm{~S} 361.0744$, found 361.0741 .

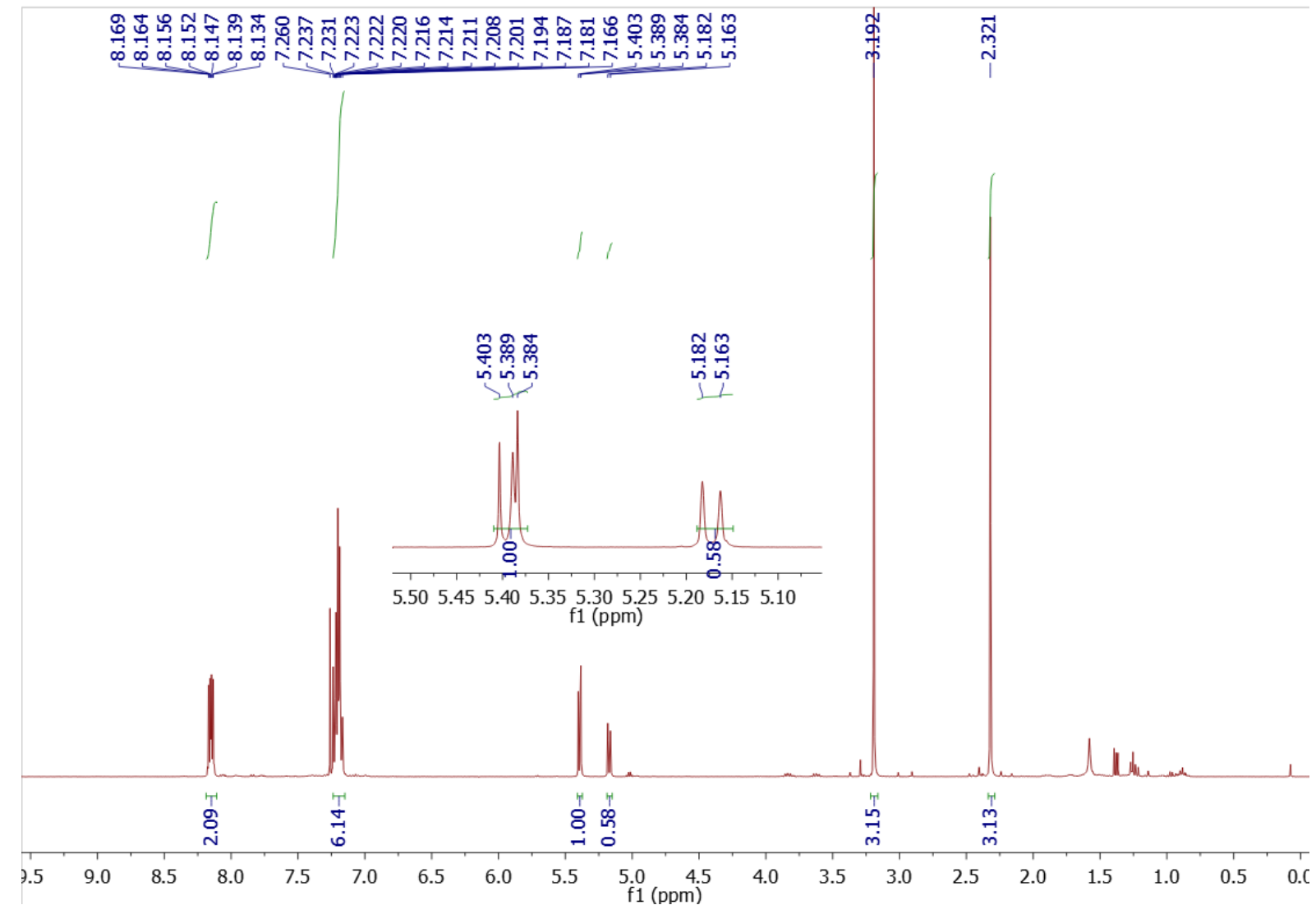


4-Fluoro-benzaldehyde $\mathbf{1 i}(0.3 \mathrm{mmol}, 37.3 \mathrm{mg})$ and $N$-methyl- $N$-tosyl-propiolamide $\mathbf{2 a}(0.1$ mmol, $23.8 \mathrm{mg}$ ) were subjected to the standard photo-mediated conditions, using $\mathrm{CH}_{3} \mathrm{CN} / \mathrm{D}_{2} \mathrm{O}$ $(1 / 1,1 \mathrm{~mL})$ as solvent. The corresponding product was obtained in $67 \%$ yield. Deuterium incorporation is $98 \%$ at the tertiary benzylic carbon according to ${ }^{1} \mathrm{H}$ NMR analysis (eq S9).
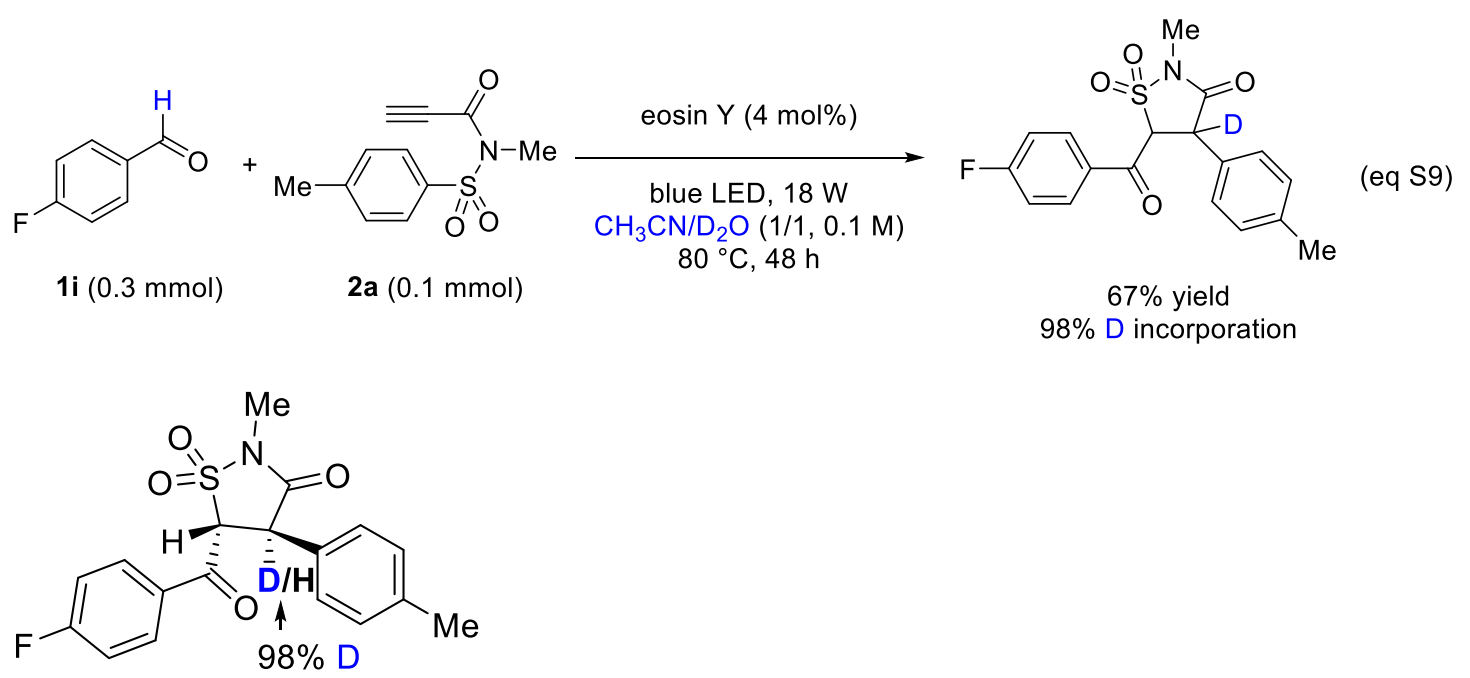

( \pm )-5-(4-Fluorobenzoyl)-2-methyl-4-( $p$-tolyl)isothiazolidin-3-one-4-d 1,1-dioxide. ${ }^{1} \mathrm{H}$ NMR $\left(400 \mathrm{MHz}, \mathrm{CDCl}_{3}\right) \delta 8.18-8.07(\mathrm{~m}, 2 \mathrm{H}), 7.24-7.12(\mathrm{~m}, 6 \mathrm{H}), 5.39-5.38(\mathrm{~m}, 1 \mathrm{H}), 5.17(\mathrm{~d}, J$ $=7.9 \mathrm{~Hz}, 0.02 \mathrm{H}), 3.19(\mathrm{~s}, 3 \mathrm{H}), 2.32(\mathrm{~s}, 3 \mathrm{H}) .{ }^{13} \mathrm{C} \mathrm{NMR}\left(126 \mathrm{MHz}, \mathrm{CDCl}_{3}\right) \delta 183.40,167.14$ $\left(\mathrm{d},{ }^{1} J_{\mathrm{C}-\mathrm{F}}=259.8 \mathrm{~Hz}\right), 166.72,139.00,132.45\left(\mathrm{~d},{ }^{3} J_{\mathrm{C}-\mathrm{F}}=10.0 \mathrm{~Hz}\right), 131.84\left(\mathrm{~d},{ }^{4} J_{\mathrm{C}-\mathrm{F}}=2.9 \mathrm{~Hz}\right)$, 131.00, 130.25, 128.57, $116.75\left(\mathrm{~d},{ }^{2} J_{\mathrm{C}-\mathrm{F}}=22.4 \mathrm{~Hz}\right), 69.96,49.82\left(\mathrm{t},{ }^{1} J_{\mathrm{C}-\mathrm{D}}=20.7 \mathrm{~Hz}\right), 25.10$, 21.24. ${ }^{19} \mathrm{~F}$ NMR $\left(377 \mathrm{MHz}, \mathrm{CDCl}_{3}\right) \delta$-100.53. HRMS ESI $[\mathrm{M}-\mathrm{H}]^{-}$calculated for $\mathrm{C}_{18} \mathrm{H}_{14} \mathrm{DFNO}_{4} \mathrm{~S} 361.0744$, found 361.0745.

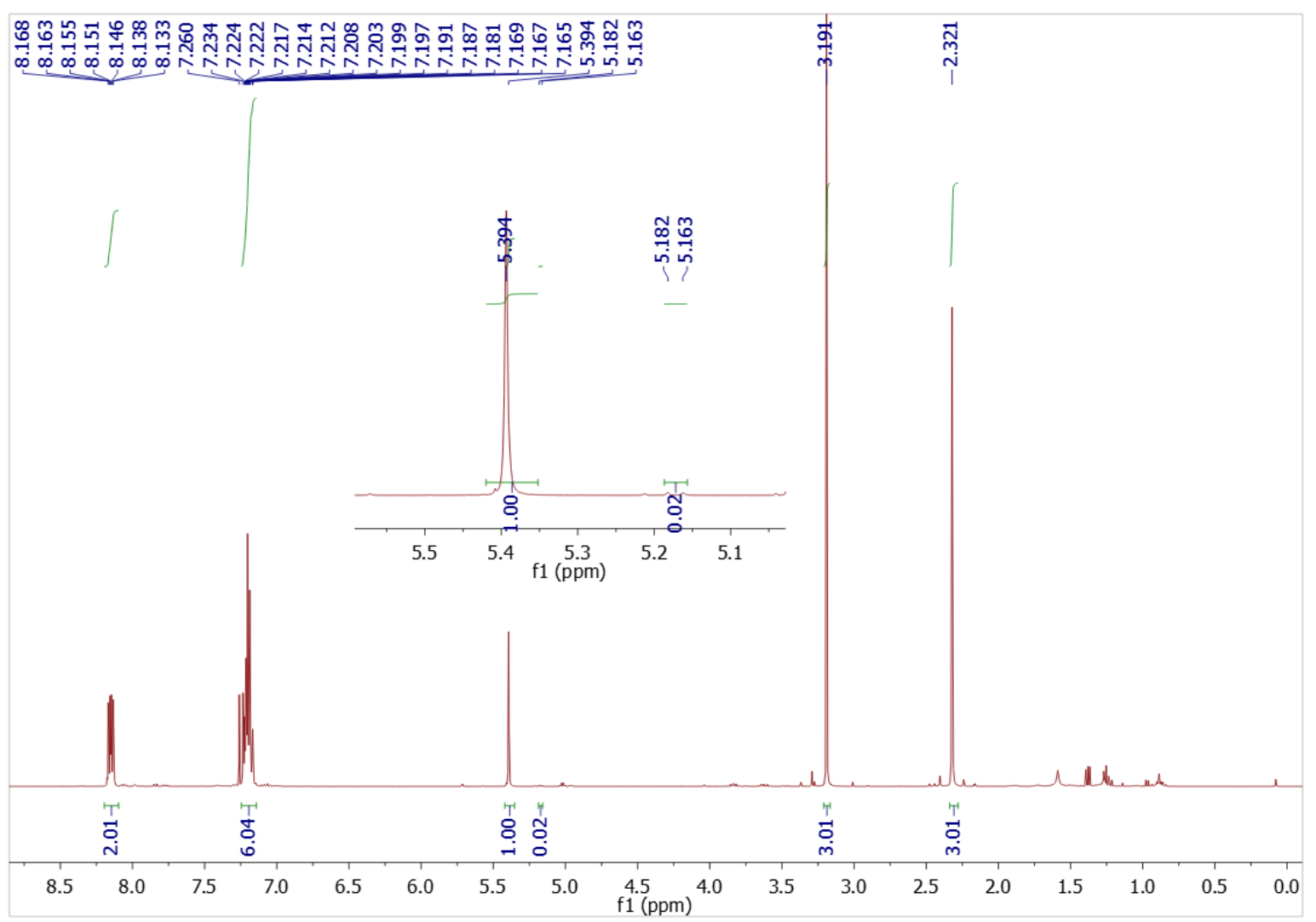


4-Fluoro-benzaldehyde $\mathbf{1 i}(0.3 \mathrm{mmol}, 37.3 \mathrm{mg})$ and $N$-methyl- $N$-tosyl-propiolamide $2 \mathrm{a}$ (23.8 $\mathrm{mg}, 0.1 \mathrm{mmol})$ were subjected to the standard photo-mediated conditions, using $\mathrm{CD}_{3} \mathrm{CN}(1 \mathrm{~mL})$ as solvent. The corresponding product 3i was obtained in $71 \%$ yield. Deuterium incorporation is $0 \%$ at the tertiary benzylic carbon according to ${ }^{1} \mathrm{H}$ NMR analysis (eq S10). This indicated no $\mathrm{H}-\mathrm{D}$ exchange between solvent $\mathrm{CD}_{3} \mathrm{CN}$ and product $\mathbf{3 i}$.
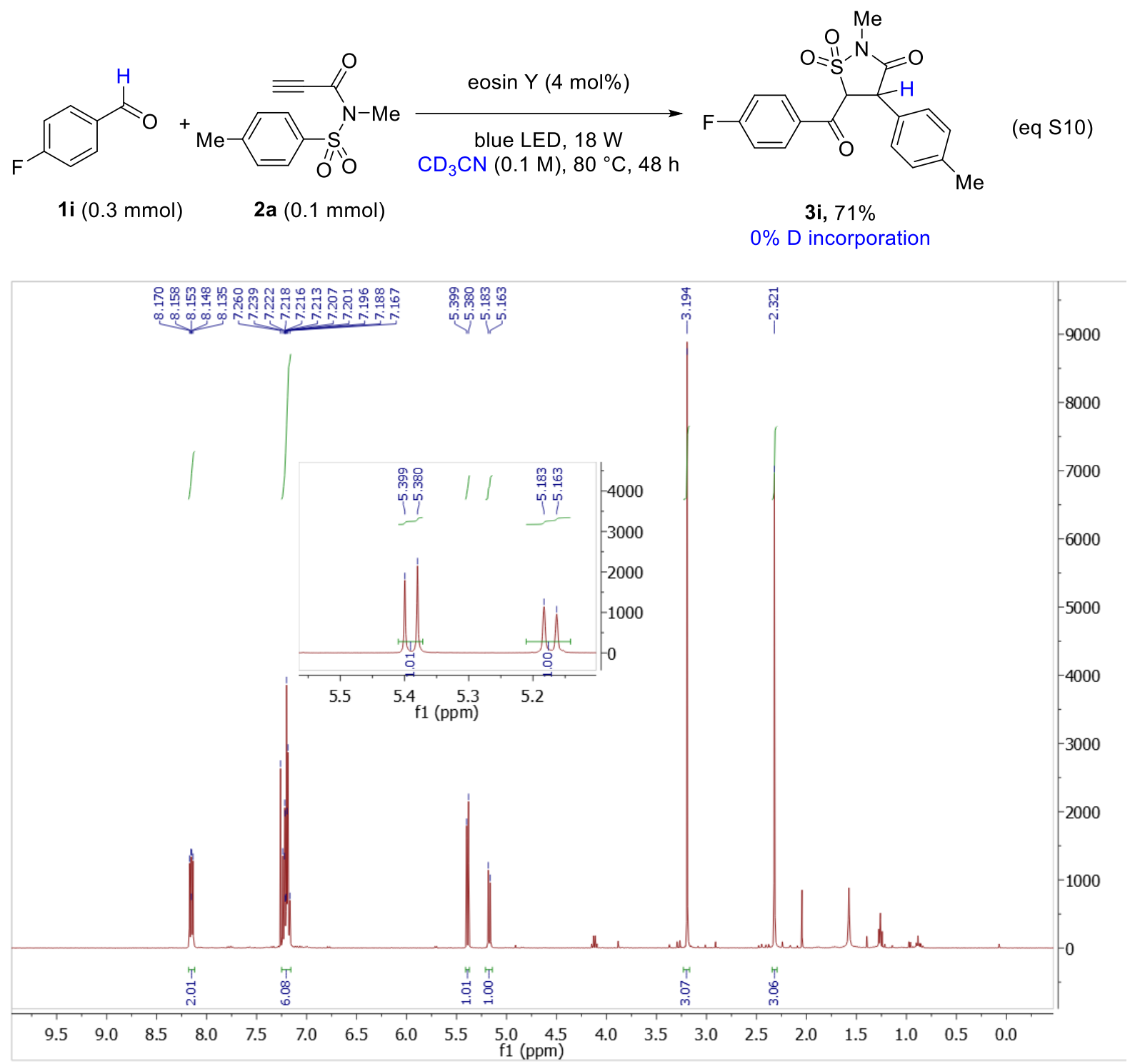

5-(4-Fluorobenzoyl)-2-methyl-4-( $p$-tolyl)isothiazolidin-3-one-1,1-dioxide 3i (0.06 mmol, 21.7 $\mathrm{mg}$ ) was subjected to the standard photo-mediated conditions, using $\mathrm{CH}_{3} \mathrm{CN} / \mathrm{D}_{2} \mathrm{O}(1 / 1,0.6 \mathrm{~mL})$ as solvent. The corresponding product was obtained in $93 \%$ yield. Deuterium incorporation is $71 \%$ at the tertiary benzylic carbon according to ${ }^{1} \mathrm{H}$ NMR analysis (eq S11).<smiles>Cc1ccc(C2C(=O)N(C)S(=O)(=O)C2C(=O)c2ccc(F)cc2)cc1</smiles>

$3 \mathbf{i}(0.06 \mathrm{mmol})$

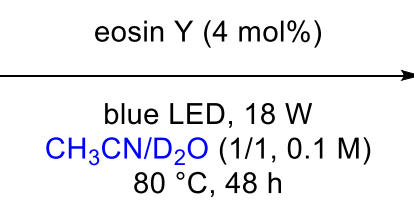

$80{ }^{\circ} \mathrm{C}, 48 \mathrm{~h}$

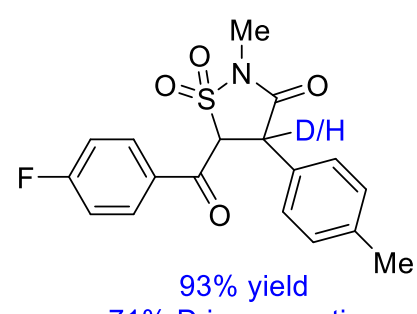

$71 \%$ D incorporation

(eq S11) 


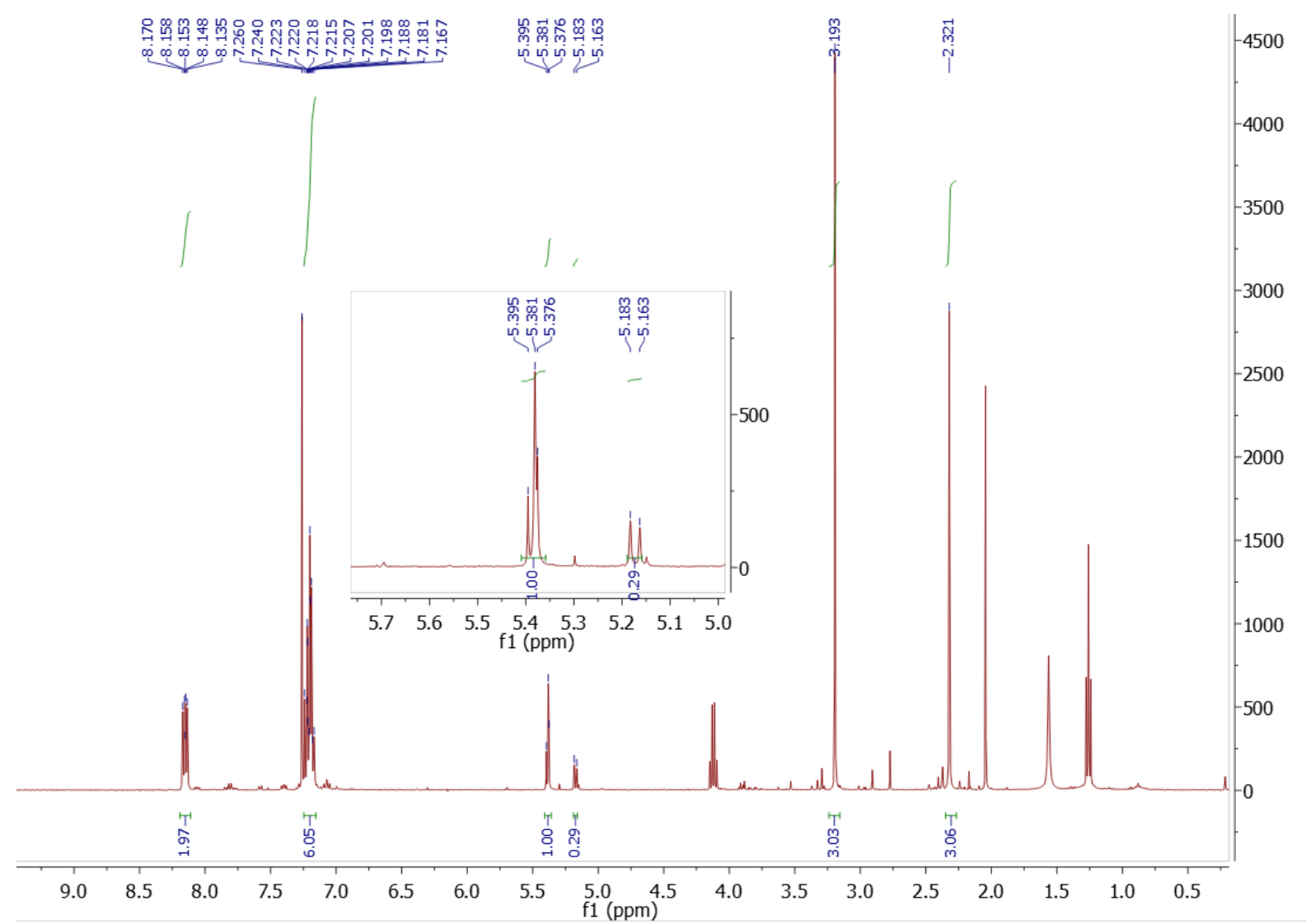

\section{Possible reason for deuterium loss}

The presence of $\mathrm{D}_{2} \mathrm{O}$ in our catalytic system led to incorporation of deuterium into the final product (eq S9-S10). This is reminiscent of TBADT (a direct HAT catalyst) which has been reported to undergo $\mathrm{H}-\mathrm{D}$ exchange with $\mathrm{D}_{2} \mathrm{O}$ in its catalytic cycle. ${ }^{8}$ As such, the deuterium loss $(42 \% \mathrm{D}$ incorporation to tertiary benzylic carbon in the product, eq S8) observed with deuterated 4-fluoro-benzaldehyde $\mathbf{1 i}-d$ can be rationalized as shown in Figure S5. Eosin Y is proposed to be a HAT catalyst. $\mathrm{D}$ atom transfer is followed by D-H exchange with $\mathrm{H}_{2} \mathrm{O}$ existed in the reaction mixture. This results in the loss of deuterium on catalytic eosin Y-D. On the other hand, deuterium loss may also occur after product formation since facile $\mathrm{H}-\mathrm{D}$ exchange was observed between $3 \mathbf{i}$ and $\mathrm{D}_{2} \mathrm{O}$ under the reaction conditions (eq S11). This $\mathrm{H}-\mathrm{D}$ exchange can be rationalized by that the eosin $\mathrm{Y}$ can behave as a photoacid under light irradiation. ${ }^{9}$ 


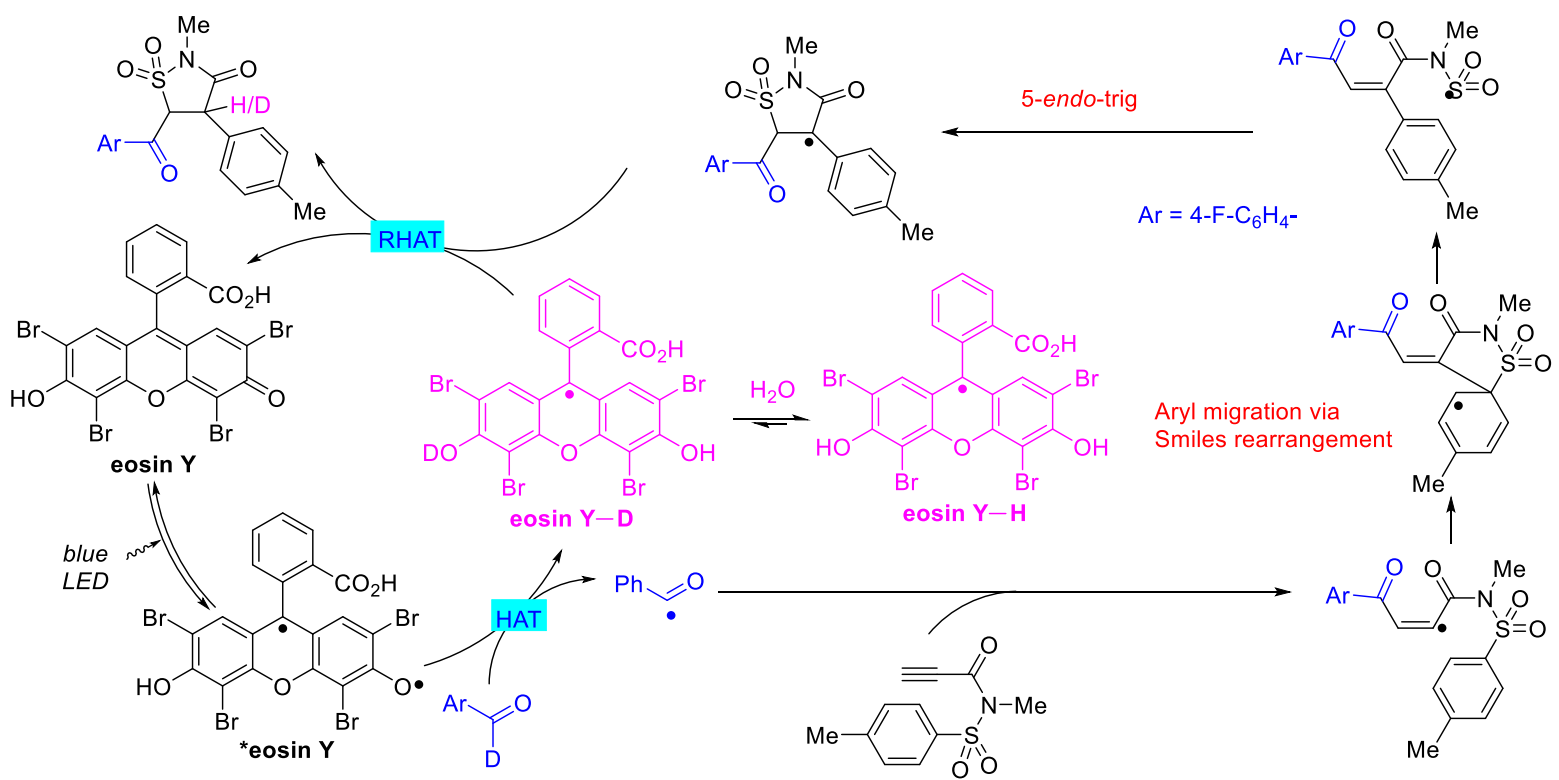

Figure S5. Possible explanation for deuterium loss.

\section{Determination of the quantum yield by standard ferrioxalate actinometry}

Determination of the light intensity at $470 \mathrm{~nm}$ : Following Yoon's procedure, ${ }^{10}$ the photon flux of the spectrophotometer was determined by standard ferrioxalate actinometry. A $0.15 \mathrm{M}$ solution of ferrioxalate was prepared by dissolving $2.21 \mathrm{~g}$ of potassium ferrioxalate hydrate in $30 \mathrm{~mL}$ of $0.05 \mathrm{M} \mathrm{H}_{2} \mathrm{SO}_{4}$. A buffered solution of phenanthroline was prepared by dissolving 50 $\mathrm{mg}$ of phenanthroline and $11.25 \mathrm{~g}$ of sodium acetate in $50 \mathrm{~mL}$ of $0.5 \mathrm{M} \mathrm{H}_{2} \mathrm{SO}_{4}$. Both solutions were stored in the dark. To determine the photon flux of the spectrophotometer, $2.0 \mathrm{~mL}$ of the ferrioxalate solution was placed in a cuvette and irradiated for 90.0 seconds at $\lambda=470 \mathrm{~nm}$ with an emission slit width at $10.0 \mathrm{~nm}$. After irradiation, $0.35 \mathrm{~mL}$ of the phenanthroline solution was added to the cuvette. The solution was then allowed to rest for $1 \mathrm{~h}$ to allow the ferrous ions to completely coordinate to the phenanthroline. The absorbance of the solution was measured at $510 \mathrm{~nm}$. A non-irradiated sample was also prepared and the absorbance at $510 \mathrm{~nm}$ measured. Conversion was calculated using eq S12.

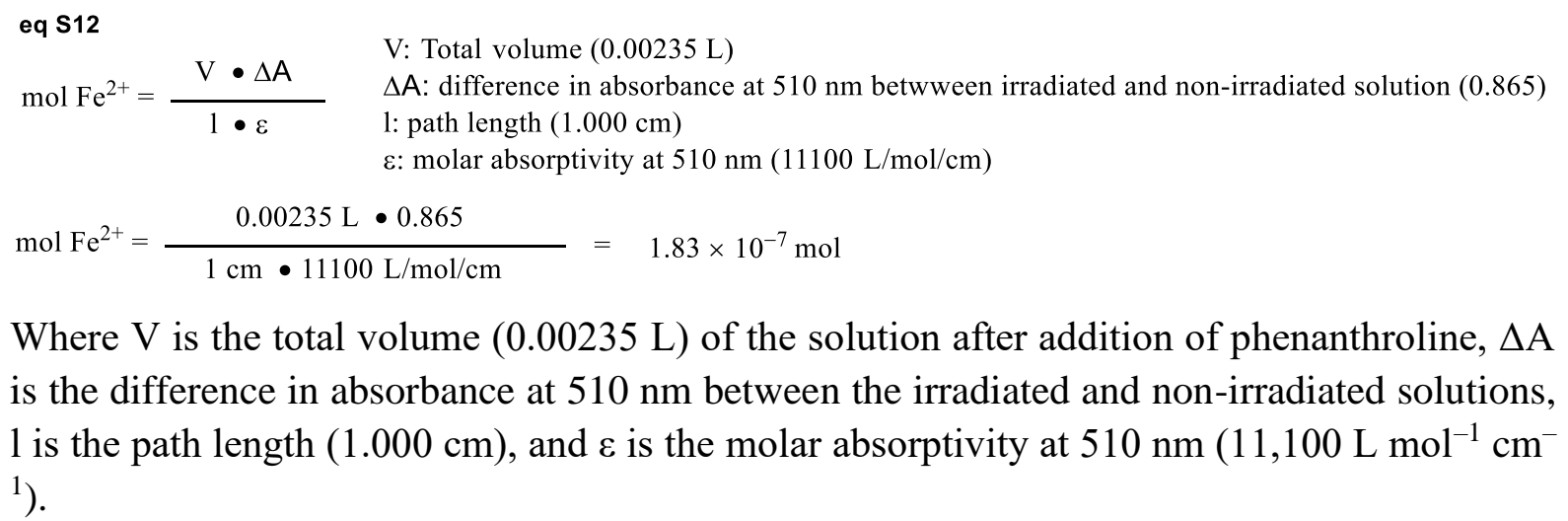

Where $\mathrm{V}$ is the total volume $(0.00235 \mathrm{~L})$ of the solution after addition of phenanthroline, $\Delta \mathrm{A}$ is the difference in absorbance at $510 \mathrm{~nm}$ between the irradiated and non-irradiated solutions, 1 is the path length $(1.000 \mathrm{~cm})$, and $\varepsilon$ is the molar absorptivity at $510 \mathrm{~nm}\left(11,100 \mathrm{~L} \mathrm{~mol}^{-1} \mathrm{~cm}^{-}\right.$ $1)$.

The photon flux can be calculated using eq S13. 
eq $\mathbf{S 1 3}$

$\begin{array}{ll}\text { Photon flux }=\frac{\mathrm{mol} \mathrm{Fe}^{2+}}{\Phi \cdot \mathrm{t} \bullet \mathrm{f}} & \begin{array}{l}\Phi: \text { Quantum yield for the ferrioxalate actinometer }(0.92 \text { for } 0.15 \mathrm{M} \text { solution at } \lambda=468 \mathrm{~nm}) \\ \text { t: irradiation time at } 470 \mathrm{~nm}(90.0 \mathrm{~s}) \\ \text { f: fraction of light at } \lambda=470 \mathrm{~nm}\left(0.735, \mathrm{f}=1-10^{-\mathrm{A}}\right)\end{array}\end{array}$

Photon flux $=\frac{1.83 \times 10^{-7} \mathrm{~mol}}{0.92 \cdot 90.0 \bullet 0.735}=3.01 \times 10^{-9}$ einstein $^{-1}$

Where $\Phi$ is the quantum yield for the ferrioxalate actinometer $(0.92$ for a $0.15 \mathrm{M}$ solution at $\lambda$ $=468 \mathrm{~nm}),{ }^{11} \mathrm{t}$ is the time $(90.0 \mathrm{~s})$, and $\mathrm{f}$ is the fraction of light absorbed at $\lambda=470 \mathrm{~nm}(0.725)$. The photon flux was calculated (average of three experiments) to be $3.01 \times 10^{-9}$ einstein s ${ }^{-1}$.

\section{Determination of quantum yield:}

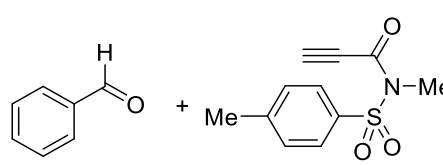

1a $(0.6 \mathrm{mmol})$

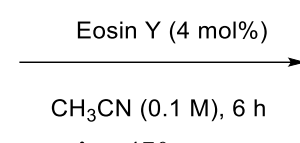

$\lambda=470 \mathrm{~nm}$

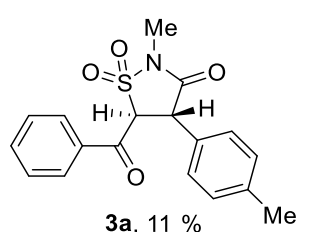

3a $11 \% \quad \mathrm{Me}$

A cuvette was charged with $N$-methyl- $N$-tosyl-propiolamide 2 a (47.6 $\mathrm{mg}, 0.2 \mathrm{mmol}$ ), benzaldehyde 1a (62 uL, $0.6 \mathrm{mmol})$, eosin Y $(5.2 \mathrm{mg}, 0.008 \mathrm{mmol})$, in anhydrous acetonitrile (2 mL). The cuvette was then capped with a PTFE stopper. The sample was stirred and irradiated $(\lambda=470 \mathrm{~nm}$, slit width $=10.0 \mathrm{~nm})$ for $14400 \mathrm{~s}(4 \mathrm{~h})$. After irradiation, the solvent was removed. The yield of product formed was determined as $11 \%$ by crude ${ }^{1} \mathrm{H}$ NMR based on a 1,3,5-trimethoxylbenzene standard. The quantum yield was determined using eq S14. Essentially all incident light $(\mathrm{f}>0.999)$ is absorbed by the eosin $\mathrm{Y}$ at the reaction conditions described above.

eq $\mathrm{S} 14$

$$
\Phi=\frac{\text { mol product }}{\text { flux } \bullet \mathrm{t} \bullet \mathrm{f}}=\frac{1.1 \times 10^{-5} \mathrm{~mol}}{3.01 \times 10^{-9} \text { einstein s}^{-1} \bullet 21600 \mathrm{~s} \bullet 1.00}=0.17
$$

$\Phi(11 \%)=0.17$, radical chain mechanism is not suggested

\section{Light on/off experiments over time}

To examine the impact of light, we conducted experiments under alternating periods of irradiation and darkness (Figure S6). These resulted in a total interruption of the reaction progress in the absence of light and recuperation of reactivity on further illumination, which allows precise temporal control over the entire reaction period. These results demonstrate that light is a necessary component of the reaction. Even though they do not definitively rule out a radical-chain process, the data show that any chain-propagation process must be short-lived. 


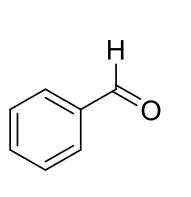

1 a $(0.3 \mathrm{mmol})$

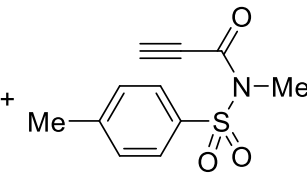

2a $(0.1 \mathrm{mmol})$
Eosin $\mathrm{Y}(4 \mathrm{~mol} \%)$

blue LED, $18 \mathrm{~W}$

$\mathrm{CH}_{3} \mathrm{CN}(0.1 \mathrm{M}), 80^{\circ} \mathrm{C}$

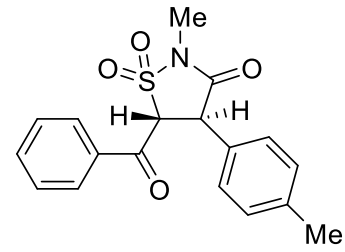

3a

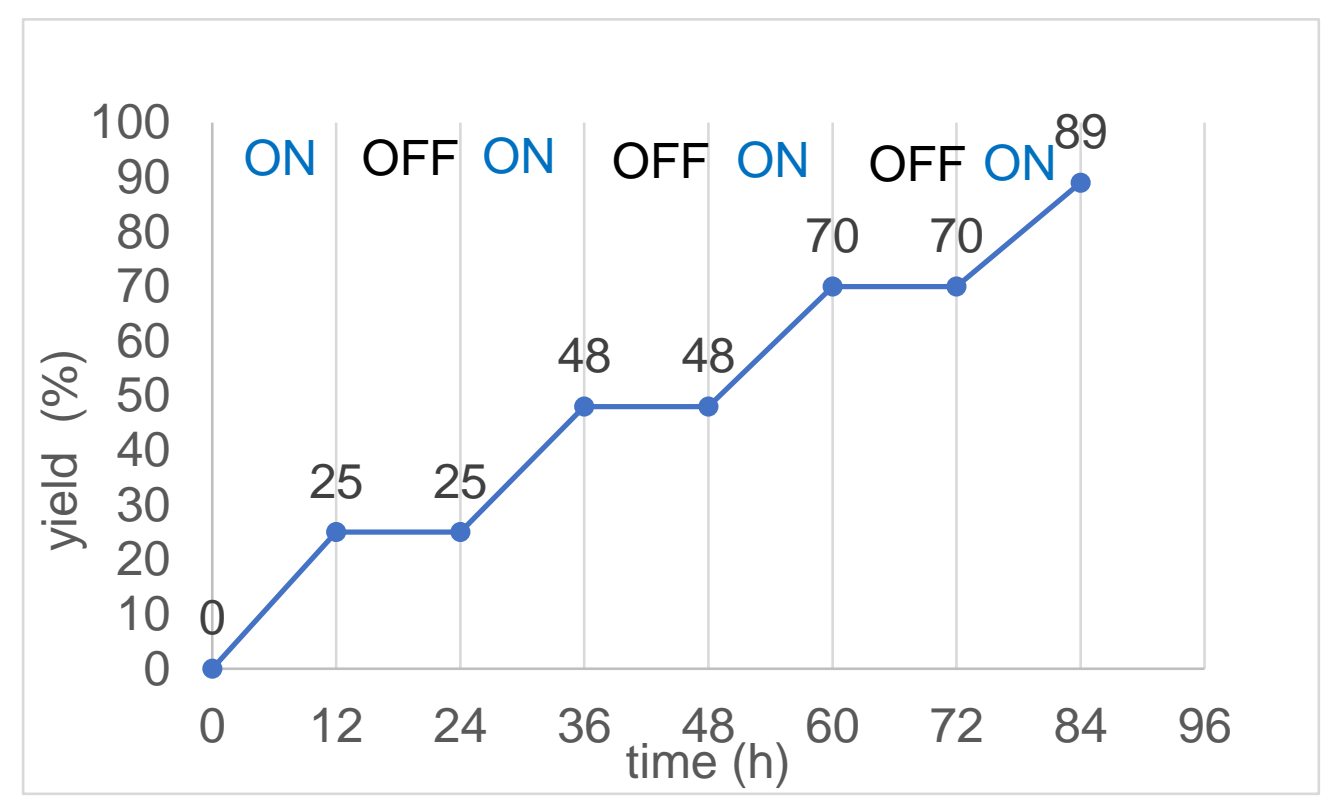

Figure S6. Time profile of the transformation with the light ON/OFF over time.

\section{Luminescence quenching experiments}

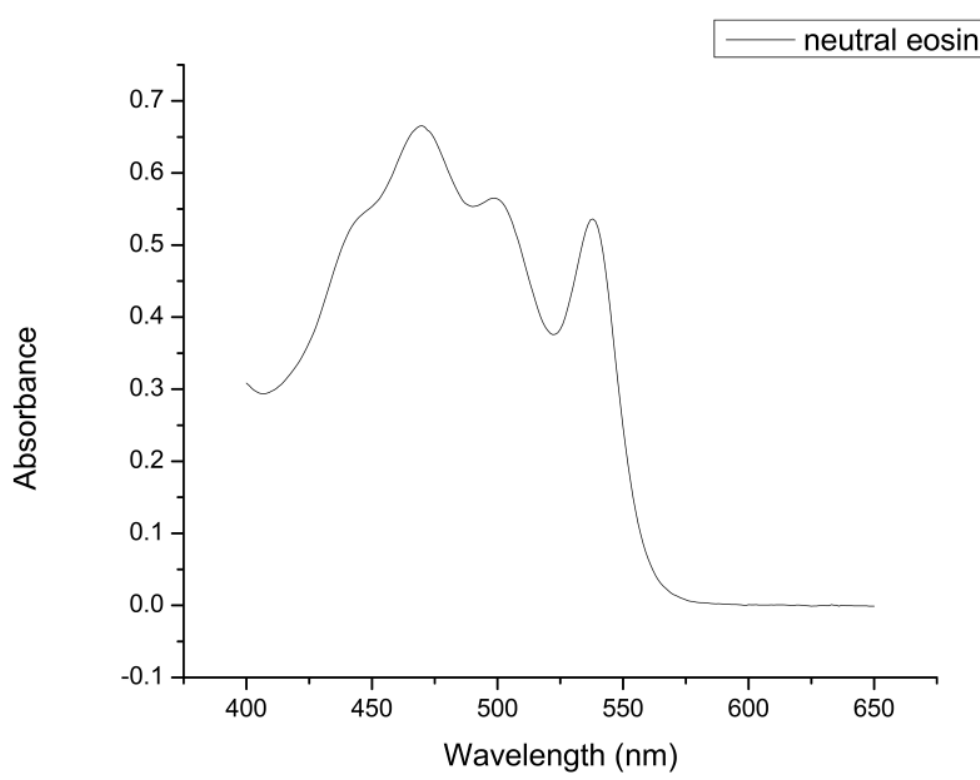

Figure S7. UV-Vis absorption of eosin $\mathrm{Y}\left(0.4 \mathrm{mM}\right.$ in $\left.\mathrm{CH}_{3} \mathrm{CN}\right)$. 


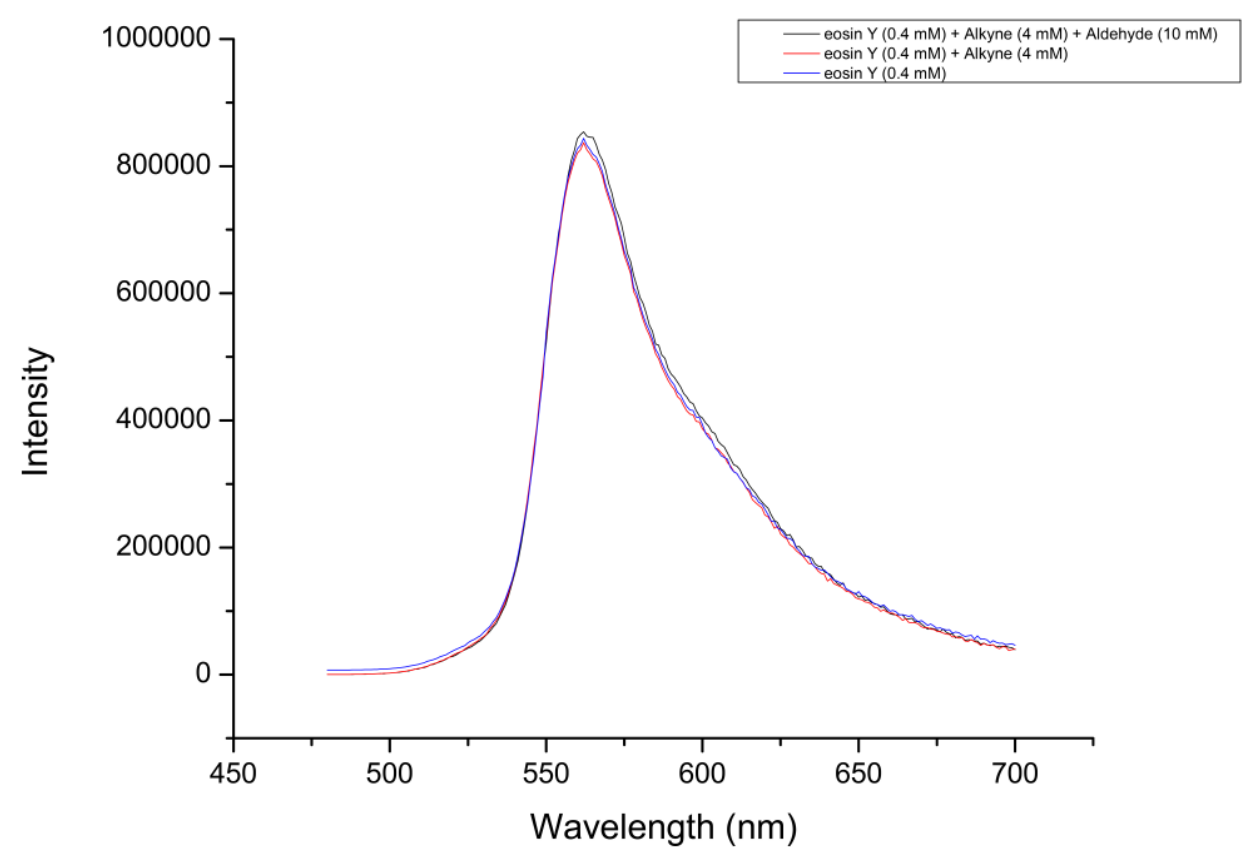

Figure S8. Fluorescence quenching study. Excited at $470 \mathrm{~nm}$. blue line: eosin $\mathrm{Y}(0.4 \mathrm{mM}$ in $\left.\mathrm{CH}_{3} \mathrm{CN}\right)$; red line: eosin $\mathrm{Y}\left(0.4 \mathrm{mM}\right.$ in $\left.\mathrm{CH}_{3} \mathrm{CN}\right)+N$-methyl- $N$-tosyl-propiolamide 2a (4 mM in $\left.\mathrm{CH}_{3} \mathrm{CN}\right)$; cyan line: eosin $\mathrm{Y}\left(0.4 \mathrm{mM}\right.$ in $\left.\mathrm{CH}_{3} \mathrm{CN}\right)+N$-methyl- $N$-tosyl-propiolamide 2a (4 $\mathrm{mM}$ in $\left.\mathrm{CH}_{3} \mathrm{CN}\right)+4$-fluoro-benzaldehyde $\mathbf{1 i}\left(10 \mathrm{mM}\right.$ in $\left.\mathrm{CH}_{3} \mathrm{CN}\right)$.

\section{DFT calculation on SET and RHAT pathways for eosin Y regeneration}

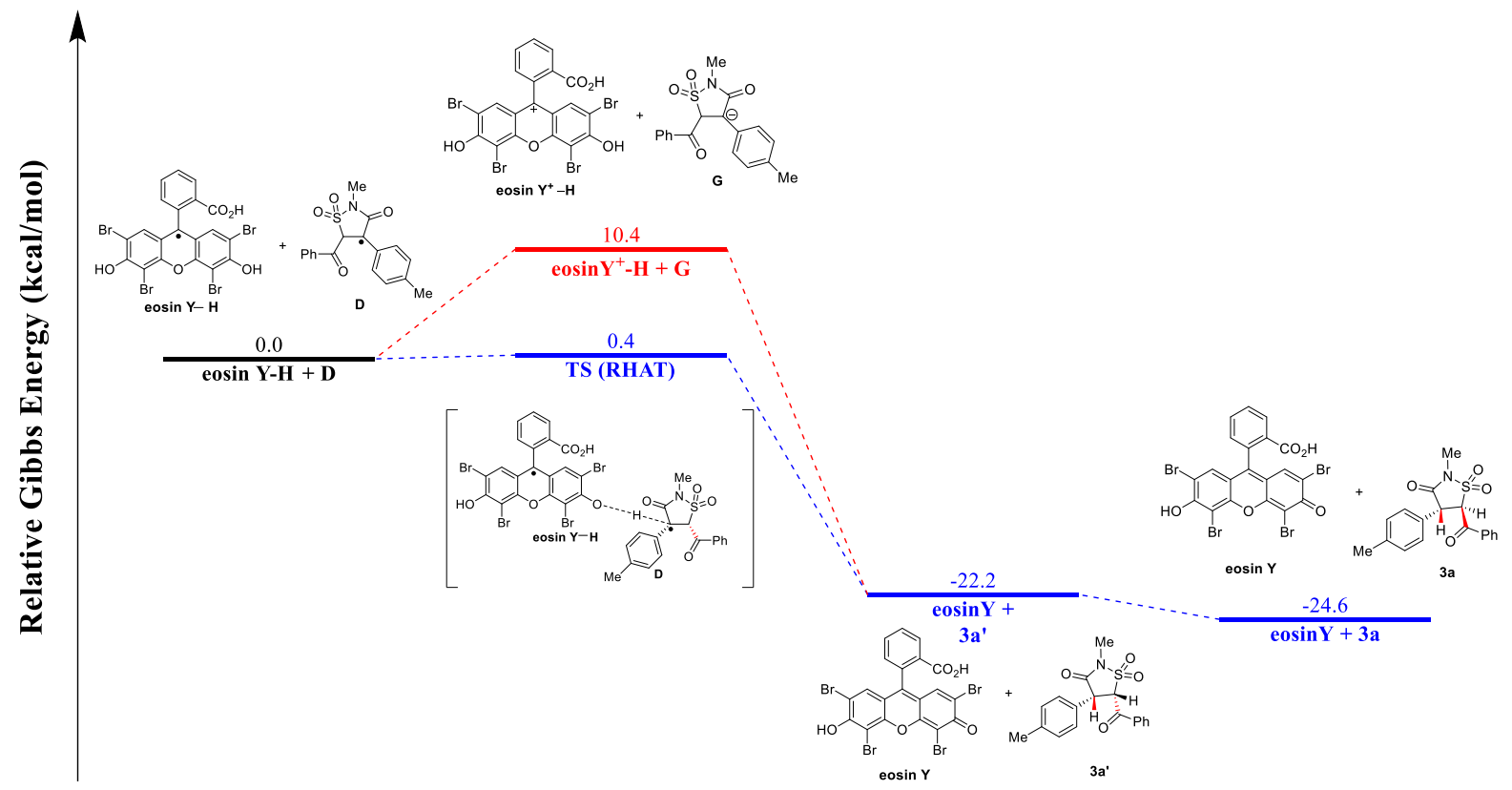

Figure S9. Density functional theory (DFT) calculations on eosin Y regeneration. 


\section{Computational details}

Density functional theory (DFT) calculations were performed to shed light on the mechanism of eosin Y regeneration (Figure S9). Reverse hydrogen atom transfer (RHAT, blue line) is the favored pathway, which features a barrier $10 \mathrm{kcal} / \mathrm{mol}$ lower than an alternative single electron transfer (SET, red line). The geometries optimization in this study was performed at the B3LYP-D3(BJ)/def2-SVP ${ }^{12,13}$ level of theory. The free energies of the optimized geometries were calculated at the same level of theory, considering the solvent effect of acetonitrile using Solvent Polarizable Continuum Model (PCM). Intrinsic reaction coordinate (IRC) calculations were performed on the transition state to verify whether it is connected with correct reactants and products or intermediates. All calculations were performed using the Gaussian 16 Rev. A.03 software suite. ${ }^{14}$

\section{Calculated Cartesian Coordinates}

\section{Structure 1. eosin Y-H}

$\mathrm{C}$

$-0.32229604$

3.25985846

$-1.61763141$

$\mathrm{C}$

0.09702530

$2.66458081-0.41491835$

C

0.64527459

3.49905872

0.58944394

C

0.79304807

4.87494103

0.35095913

0.37139530

5.44529422

$-0.84747931$

C

$-0.19628983$

4.63255070

$-1.83321368$

$-0.73802373$

2.62257660

$-2.40071812$

$\mathrm{H}$

1.23407322

5.48978945

1.13667620

0.48312295

6.51880308

$-1.01229171$

$-0.53039725$

5.06522013

$-2.77884252$

1.08637415

3.01965383

1.93545277

1.86832383

3.62287448

2.63944622

0.50893429

1.87434882

2.32428140

0.86577142

1.64844389

3.20170189

$-0.00251730$

1.19191254

$-0.28919739$

1.17587110

0.37603010

$-0.25653317$

2.48967023

0.88990575

$-0.33689554$

1.03738639

$-1.03214532$

$-0.14492734$

3.58815723

0.04471497

$-0.28497179$

2.64190974

1.96268650

$-0.44349862$

2.14628813

$-1.87669840$

$-0.09602944$

3.45531490

$-1.35403222$

$-0.16013332$

$-1.27782822$

0.54634838

$-0.21091593$

$-2.51345513$

1.23571386

$-0.19483133$

$-1.32533839$

$-0.86810784$

$-0.11188730$

$-3.71861246$

0.55398400

$-0.11010424$

$\mathrm{H}$

$-2.51648319$

2.32322162

$-0.24666958$

C

$-0.02825162$ 


$\begin{array}{lccc}\mathrm{C} & -3.76958073 & -0.85326881 & -0.02944627 \\ \mathrm{Br} & 1.90613289 & -3.75093146 & 0.05915201 \\ \mathrm{O} & 4.49475932 & -2.19706099 & -0.10444947 \\ \mathrm{H} & 5.32439442 & -1.69036140 & -0.15923717 \\ \mathrm{Br} & 5.35317938 & 0.76802738 & -0.38503427 \\ \mathrm{Br} & -5.35296464 & 1.52923097 & -0.09659478 \\ \mathrm{O} & -4.95515335 & -1.47131259 & 0.04718330 \\ \mathrm{H} & -4.81740986 & -2.43380169 & 0.10035301 \\ \mathrm{O} & -0.19058755 & -1.61488105 & -0.08622149 \\ \mathrm{Br} & -2.56571390 & -3.44464595 & 0.09646781\end{array}$

\section{Structure 2. Compound D}

$\begin{array}{lrrc}\mathrm{C} & -4.36695542 & -1.84715025 & -0.26779471 \\ \mathrm{C} & -4.53824995 & -0.50147746 & 0.12080391 \\ \mathrm{C} & -3.47017990 & 0.37566838 & 0.19819900 \\ \mathrm{C} & -3.06394531 & -2.27897915 & -0.57982173 \\ \mathrm{H} & -5.54083071 & -0.14250522 & 0.36784059 \\ \mathrm{H} & -3.62380369 & 1.40780029 & 0.50455646 \\ \mathrm{H} & -2.90131017 & -3.31575504 & -0.88364915 \\ \mathrm{C} & -5.54306607 & -2.77869145 & -0.34041613 \\ \mathrm{H} & -6.03216835 & -2.86986365 & 0.64385725 \\ \mathrm{H} & -6.30597503 & -2.39469198 & -1.03785839 \\ \mathrm{H} & -5.24669282 & -3.78357603 & -0.67182211 \\ \mathrm{C} & -2.14544976 & -0.05429517 & -0.11662790 \\ \mathrm{~S} & 1.37498329 & 1.84836361 & -0.52428270 \\ \mathrm{O} & 1.53339930 & 2.20954129 & -1.93523310 \\ \mathrm{O} & 2.56896545 & 1.81050642 & 0.32523683 \\ \mathrm{~N} & 0.20660073 & 2.81516197 & 0.21879930 \\ \mathrm{C} & 0.49497267 & 4.20773474 & 0.51861583 \\ \mathrm{H} & 0.66472350 & 4.78339009 & -0.40414672 \\ \mathrm{H} & -0.38236171 & 4.60888850 & 1.04078773 \\ \mathrm{H} & 1.37957597 & 4.27800526 & 1.16733128 \\ \mathrm{C} & -1.02189365 & 0.81723512 & -0.04803516 \\ \mathrm{C} & -1.05952722 & 2.22839033 & 0.36187519 \\ \mathrm{O} & -2.01236098 & 2.86773236 & 0.78027037 \\ \mathrm{C} & 0.35976301 & 0.32809112 & -0.37124036 \\ \mathrm{H} & 0.41726160 & -0.14708760 & -1.35879997 \\ \mathrm{C} & 0.98225301 & -0.57715162 & 0.72384341 \\ \mathrm{O} & 0.48232587 & -0.59561258 & 1.82874624 \\ \mathrm{C} & 2.16130111 & -1.39885566 & 0.35246834 \\ \mathrm{C} & 2.81782613 & -1.27501805 & -0.88529426 \\ \mathrm{C} & 2.64159307 & -2.32203421 & 1.29957951 \\ \mathrm{C} & 3.93414592 & -2.06236719 & -1.16770637 \\ \mathrm{H} & 2.48309341 & -0.55787794 & -1.63562293 \\ & & & \\ \mathrm{H} & & & \\ \mathrm{H} & & \end{array}$




$\begin{array}{lrrr}\mathrm{C} & 3.75087521 & -3.11233217 & 1.01166346 \\ \mathrm{H} & 2.12561943 & -2.40274920 & 2.25735721 \\ \mathrm{C} & 4.39901876 & -2.98295728 & -0.22325152 \\ \mathrm{H} & 4.44227526 & -1.95660916 & -2.12814428 \\ \mathrm{H} & 4.11559142 & -3.83079237 & 1.74876667 \\ \mathrm{H} & 5.27057867 & -3.60141082 & -0.44942443 \\ \mathrm{C} & -1.98197888 & -1.41465076 & -0.50930120 \\ \mathrm{H} & -0.99316775 & -1.79941230 & -0.76161946\end{array}$

\section{Structure 3. eosin $\mathrm{Y}^{+}-\mathrm{H}$}

$\begin{array}{lrrc}\mathrm{C} & -0.08358901 & 3.11287885 & -1.75911095 \\ \mathrm{C} & 0.03121427 & 2.63199723 & -0.44691951 \\ \mathrm{C} & 0.18541676 & 3.54712931 & 0.61819269 \\ \mathrm{C} & 0.23004667 & 4.92047153 & 0.33757460 \\ \mathrm{C} & 0.12080854 & 5.39069419 & -0.96902346 \\ \mathrm{C} & -0.03837963 & 4.48388592 & -2.01936560 \\ \mathrm{H} & -0.20319337 & 2.40441931 & -2.58069339 \\ \mathrm{H} & 0.34861351 & 5.60923232 & 1.17470620 \\ \mathrm{H} & 0.15687324 & 6.46323762 & -1.16809356 \\ \mathrm{H} & -0.12744822 & 4.83935520 & -3.04776148 \\ \mathrm{C} & 0.29108080 & 3.15347780 & 2.05667016 \\ \mathrm{O} & 0.47824224 & 3.93714969 & 2.95880608 \\ \mathrm{O} & 0.14937834 & 1.83474269 & 2.25577054 \\ \mathrm{H} & 0.23348202 & 1.65360852 & 3.20880638 \\ \mathrm{C} & 0.00124141 & 1.15486610 & -0.27035705 \\ \mathrm{C} & 1.20487788 & 0.41510929 & -0.23213558 \\ \mathrm{C} & 2.48834871 & 1.02217820 & -0.28840691 \\ \mathrm{C} & 1.13448746 & -1.00237781 & -0.11874184 \\ \mathrm{C} & 3.61884737 & 0.24809848 & -0.22606484 \\ \mathrm{H} & 2.56422139 & 2.10449022 & -0.37994585 \\ \mathrm{C} & 2.28395673 & -1.79268550 & -0.05868351 \\ \mathrm{C} & -5.54849590 & -1.17914148 & -0.10846224 \\ \mathrm{C} & -1.23071140 & 0.46767438 & -0.21600187 \\ \mathrm{C} & -2.49185943 & 1.12537955 & -0.24386255 \\ \mathrm{C} & -1.21306665 & -0.95163887 & -0.10948683 \\ \mathrm{C} & -3.65755557 & 0.40441234 & -0.17510664 \\ \mathrm{H} & -2.52228850 & 2.21126615 & -0.31819629 \\ \mathrm{C} & -2.39672492 & -1.68705164 & -0.04499096 \\ \mathrm{C} & -3.63784602 & -1.02517063 & -0.07517926 \\ \mathrm{Br} & 2.15036435 & -3.66727965 & 0.09203009 \\ \mathrm{O} & 4.62435665 & -1.93960053 & -0.04605385 \\ \mathrm{H} & 5.43347108 & -1.39385684 & -0.08960337 \\ & 5.34250741 & 1.03820725 & -0.29660788 \\ \mathrm{Br} & & & -0.20973611\end{array}$




$\begin{array}{lrrr}\mathrm{O} & -4.79043382 & -1.66187340 & -0.01377405 \\ \mathrm{H} & -4.64863679 & -2.62605271 & 0.05347169 \\ \mathrm{O} & -0.05455152 & -1.62376183 & -0.06438092 \\ \mathrm{Br} & -2.35529777 & -3.57328527 & 0.08634648\end{array}$

\section{Structure 4. Compound G}

\begin{tabular}{|c|c|c|c|}
\hline $\mathrm{C}$ & -4.34401168 & -1.91128101 & -0.28073500 \\
\hline $\mathrm{C}$ & -4.53210297 & -0.56498930 & 0.08667497 \\
\hline $\mathrm{C}$ & -3.47280927 & 0.33076717 & 0.18733732 \\
\hline $\mathrm{C}$ & -3.02729791 & -2.31401741 & -0.54085494 \\
\hline $\mathrm{H}$ & -5.54577783 & -0.20924152 & 0.30125866 \\
\hline $\mathrm{H}$ & -3.65016482 & 1.36616474 & 0.47585924 \\
\hline $\mathrm{H}$ & -2.83161626 & -3.35299927 & -0.82528380 \\
\hline $\mathrm{C}$ & -5.50818609 & -2.86532311 & -0.38173988 \\
\hline $\mathrm{H}$ & -6.04636649 & -2.95698472 & 0.57773400 \\
\hline $\mathrm{H}$ & -6.24950018 & -2.52851068 & -1.12720484 \\
\hline $\mathrm{H}$ & -5.17656805 & -3.87310780 & -0.67462108 \\
\hline $\mathrm{C}$ & -2.13193768 & -0.06733455 & -0.07880220 \\
\hline S & 1.33365812 & 1.89817435 & -0.50301630 \\
\hline $\mathrm{O}$ & 1.34464429 & 2.30071060 & -1.92279265 \\
\hline $\mathrm{O}$ & 2.64176027 & 1.84383397 & 0.17540434 \\
\hline $\mathrm{N}$ & 0.26029218 & 2.80593004 & 0.39851635 \\
\hline $\mathrm{C}$ & 0.40654307 & 4.25020345 & 0.37974080 \\
\hline $\mathrm{H}$ & 0.34675395 & 4.66771976 & -0.64112985 \\
\hline $\mathrm{H}$ & -0.42187653 & 4.65645478 & 0.97388280 \\
\hline $\mathrm{H}$ & 1.36521535 & 4.54001461 & 0.83406725 \\
\hline $\mathrm{C}$ & -1.01391164 & 0.84397192 & 0.01096780 \\
\hline $\mathrm{C}$ & -1.07691699 & 2.19321944 & 0.40987585 \\
\hline $\mathrm{O}$ & -2.02838973 & 2.91112936 & 0.75271935 \\
\hline $\mathrm{C}$ & 0.37138585 & 0.35695192 & -0.29885402 \\
\hline $\mathrm{H}$ & 0.45019155 & -0.14064741 & -1.27702612 \\
\hline $\mathrm{C}$ & 1.05562562 & -0.50983809 & 0.78087920 \\
\hline $\mathrm{O}$ & 0.65156378 & -0.49177223 & 1.92653989 \\
\hline $\mathrm{C}$ & 2.19184936 & -1.38598747 & 0.36554515 \\
\hline $\mathrm{C}$ & 2.83078640 & -1.26494516 & -0.88128120 \\
\hline $\mathrm{C}$ & 2.64744011 & -2.35409744 & 1.27790327 \\
\hline $\mathrm{C}$ & 3.90240807 & -2.09872089 & -1.20552213 \\
\hline $\mathrm{H}$ & 2.51668117 & -0.50952077 & -1.60156810 \\
\hline $\mathrm{C}$ & 3.71029985 & -3.19257691 & 0.94920626 \\
\hline $\mathrm{H}$ & 2.14732171 & -2.43150840 & 2.24462259 \\
\hline $\mathrm{C}$ & 4.34032301 & -3.06548901 & -0.29498165 \\
\hline $\mathrm{H}$ & 4.39769046 & -1.99241167 & -2.17300789 \\
\hline $\mathrm{H}$ & 4.05259059 & -3.94645564 & 1.66177262 \\
\hline $\mathrm{H}$ & 5.17544997 & -3.72046076 & -0.55428757 \\
\hline
\end{tabular}




$\begin{array}{llll}\mathrm{C} & -1.95233765 & -1.42753788 & -0.44222412 \\ \mathrm{H} & -0.95012530 & -1.80987267 & -0.65020172\end{array}$

\section{Structure 5. TS (RHAT)}

\begin{tabular}{|c|c|c|c|}
\hline $\mathrm{C}$ & 1.06716401 & 0.66769978 & -1.38701568 \\
\hline $\mathrm{C}$ & 0.18026510 & -0.42630584 & -1.36348934 \\
\hline $\mathrm{C}$ & -1.15055029 & -0.23360597 & -0.99741859 \\
\hline $\mathrm{C}$ & -1.68151946 & 1.07293966 & -0.75665213 \\
\hline $\mathrm{C}$ & -0.78747889 & 2.18025510 & -0.84386697 \\
\hline $\mathrm{C}$ & 0.53401938 & 1.97945172 & -1.13635220 \\
\hline $\mathrm{C}$ & -3.04908912 & 1.20918814 & -0.46314774 \\
\hline $\mathrm{C}$ & -3.85233931 & 0.03705842 & -0.35556641 \\
\hline $\mathrm{C}$ & -3.24235259 & -1.22646229 & -0.56755044 \\
\hline $\mathrm{C}$ & -3.95511417 & -2.41893597 & -0.42582973 \\
\hline $\mathrm{C}$ & -5.31862030 & -2.38344904 & -0.08294806 \\
\hline $\mathrm{C}$ & -5.93496294 & -1.11109643 & 0.10496291 \\
\hline $\mathrm{C}$ & -5.23033548 & 0.06363130 & -0.03125692 \\
\hline $\mathrm{H}$ & -1.16533353 & 3.18424719 & -0.66122927 \\
\hline $\mathrm{H}$ & -5.72930502 & 1.01992460 & 0.11626897 \\
\hline $\mathrm{C}$ & -3.66936827 & 2.55932524 & -0.38697508 \\
\hline $\mathrm{C}$ & -3.83654613 & 3.22697847 & -1.61133256 \\
\hline $\mathrm{C}$ & -4.12848457 & 3.17963700 & 0.79812899 \\
\hline $\mathrm{C}$ & -4.45425320 & 4.47672900 & -1.67165014 \\
\hline $\mathrm{H}$ & -3.48600985 & 2.74887899 & -2.52770333 \\
\hline $\mathrm{C}$ & -4.75075657 & 4.43541136 & 0.71929483 \\
\hline $\mathrm{C}$ & -4.91643088 & 5.08357643 & -0.50177492 \\
\hline $\mathrm{H}$ & -4.57421657 & 4.97267330 & -2.63690671 \\
\hline $\mathrm{H}$ & -5.09410819 & 4.89368633 & 1.64719556 \\
\hline $\mathrm{H}$ & -5.40067606 & 6.06102957 & -0.54032934 \\
\hline $\mathrm{C}$ & -3.98474389 & 2.61148516 & 2.17261009 \\
\hline $\mathrm{O}$ & -4.49501181 & 3.09440976 & 3.15768184 \\
\hline $\mathrm{O}$ & -3.21713250 & 1.51068111 & 2.22910308 \\
\hline $\mathrm{H}$ & -3.18641183 & 1.21286952 & 3.15588587 \\
\hline $\mathrm{Br}$ & 1.72481189 & 3.44969387 & -1.23773477 \\
\hline $\mathrm{Br}$ & 0.80279884 & -2.14710119 & -1.82361739 \\
\hline $\mathrm{Br}$ & -3.09102113 & -4.07928701 & -0.67552454 \\
\hline $\mathrm{Br}$ & -7.78191653 & -1.08258688 & 0.55548447 \\
\hline $\mathrm{O}$ & -1.94396242 & -1.31621748 & -0.91290739 \\
\hline $\mathrm{O}$ & -5.97443247 & -3.52767608 & 0.04814462 \\
\hline $\mathrm{H}$ & -6.90614644 & -3.35771894 & 0.28279520 \\
\hline $\mathrm{O}$ & 2.33498136 & 0.47320818 & -1.69418047 \\
\hline $\mathrm{H}$ & 2.93802425 & 1.03303646 & -1.13271656 \\
\hline $\mathrm{C}$ & -0.07970854 & -0.86547285 & 2.00379818 \\
\hline $\mathrm{C}$ & 0.15492314 & 0.51967372 & 2.080838 \\
\hline
\end{tabular}




\begin{tabular}{|c|c|c|c|}
\hline $\mathrm{C}$ & 1.38953818 & 1.07238099 & 1.75947418 \\
\hline $\mathrm{C}$ & 1.00162642 & -1.67787286 & 1.62424125 \\
\hline $\mathrm{H}$ & -0.66270301 & 1.18391737 & 2.36989207 \\
\hline $\mathrm{H}$ & 1.53821064 & 2.14930226 & 1.80898494 \\
\hline $\mathrm{H}$ & 0.86063745 & -2.75994002 & 1.56025678 \\
\hline $\mathrm{C}$ & -1.41679924 & -1.46908140 & 2.34786306 \\
\hline $\mathrm{H}$ & -1.48682576 & -1.70837699 & 3.42382973 \\
\hline $\mathrm{H}$ & -2.24137588 & -0.78134580 & 2.11641608 \\
\hline $\mathrm{H}$ & -1.58557602 & -2.40601959 & 1.79639128 \\
\hline $\mathrm{C}$ & 2.47134437 & 0.26285380 & 1.31907434 \\
\hline S & 5.85800077 & 1.11286907 & -0.58814398 \\
\hline $\mathrm{O}$ & 5.29810124 & 1.35849895 & -1.93057143 \\
\hline $\mathrm{O}$ & 7.29225982 & 0.80701731 & -0.49841605 \\
\hline $\mathrm{N}$ & 5.47172652 & 2.35519275 & 0.47417349 \\
\hline $\mathrm{C}$ & 5.98810456 & 3.68737008 & 0.21234057 \\
\hline $\mathrm{H}$ & 5.64258827 & 4.08352115 & -0.75803671 \\
\hline $\mathrm{H}$ & 5.61119488 & 4.33658331 & 1.01237572 \\
\hline $\mathrm{H}$ & 7.08676881 & 3.67432890 & 0.23323698 \\
\hline $\mathrm{C}$ & 3.71058465 & 0.81952978 & 0.83475971 \\
\hline $\mathrm{C}$ & 4.16221293 & 2.16015265 & 1.05966224 \\
\hline $\mathrm{O}$ & 3.61760408 & 3.10593646 & 1.62617813 \\
\hline $\mathrm{C}$ & 4.79616353 & -0.07655928 & 0.30346120 \\
\hline $\mathrm{H}$ & 4.44482419 & -0.77852411 & -0.46400446 \\
\hline $\mathrm{C}$ & 5.64466164 & -0.80797624 & 1.36753785 \\
\hline $\mathrm{O}$ & 5.65539764 & -0.39842383 & 2.51134147 \\
\hline $\mathrm{C}$ & 6.40577342 & -2.02002641 & 0.95359551 \\
\hline $\mathrm{C}$ & 6.54766679 & -2.41024502 & -0.39003022 \\
\hline $\mathrm{C}$ & 7.02031135 & -2.78728206 & 1.95995359 \\
\hline $\mathrm{C}$ & 7.28835068 & -3.54724467 & -0.71647481 \\
\hline $\mathrm{H}$ & 6.10059780 & -1.82765415 & -1.19519232 \\
\hline $\mathrm{C}$ & 7.75193768 & -3.92617178 & 1.63318203 \\
\hline $\mathrm{H}$ & 6.90729962 & -2.46897207 & 2.99737904 \\
\hline $\mathrm{C}$ & 7.88712887 & -4.30799706 & 0.29242484 \\
\hline $\mathrm{H}$ & 7.39836886 & -3.83988340 & -1.76270174 \\
\hline $\mathrm{H}$ & 8.22054552 & -4.51956019 & 2.42138070 \\
\hline $\mathrm{H}$ & 8.46183648 & -5.20040528 & 0.03390155 \\
\hline $\mathrm{C}$ & 2.23649592 & -1.13721366 & 1.28905483 \\
\hline $\mathrm{H}$ & 3.02878456 & -1.81645931 & 0.96989764 \\
\hline
\end{tabular}

Structure 6. Compound 3a'

$\begin{array}{lrrr}\mathrm{C} & 3.20100612 & -2.09470726 & -0.01132085 \\ \mathrm{C} & 2.32288461 & -1.90440091 & -1.09081808 \\ \mathrm{C} & 0.97489920 & -1.61392067 & -0.88590720 \\ \mathrm{C} & 2.67385162 & -2.00863140 & 1.28549561\end{array}$




$\begin{array}{lrrr}\mathrm{H} & 2.70407133 & -1.97874554 & -2.11266628 \\ \mathrm{H} & 0.32229999 & -1.46036820 & -1.74551460 \\ \mathrm{H} & 3.33001582 & -2.16475541 & 2.14553382 \\ \mathrm{C} & 4.66630719 & -2.35609944 & -0.24452154 \\ \mathrm{H} & 4.82481864 & -3.01521356 & -1.11168664 \\ \mathrm{H} & 5.20035540 & -1.41288156 & -0.45286065 \\ \mathrm{H} & 5.13990487 & -2.81658879 & 0.63466518 \\ \mathrm{C} & 0.45991602 & -1.51005689 & 0.41428986 \\ \mathrm{~S} & -2.88524234 & 0.73396812 & 0.58832698 \\ \mathrm{O} & -3.32307832 & 1.88160362 & -0.20352579 \\ \mathrm{O} & -3.45424154 & 0.53462384 & 1.92363250 \\ \mathrm{~N} & -3.08610020 & -0.68236911 & -0.34746966 \\ \mathrm{C} & -4.27358158 & -0.87099541 & -1.16456985 \\ \mathrm{H} & -4.38527708 & -0.03204132 & -1.86576446 \\ \mathrm{H} & -4.13167412 & -1.80504779 & -1.72246259 \\ \mathrm{H} & -5.17262042 & -0.95324523 & -0.53649443 \\ \mathrm{C} & -0.95349520 & -1.05187399 & 0.68323774 \\ \mathrm{C} & -2.02413782 & -1.56246576 & -0.29396031 \\ \mathrm{O} & -1.97818193 & -2.60885799 & -0.90288860 \\ \mathrm{C} & 1.32405457 & -1.72057509 & 1.49523513 \\ \mathrm{H} & -0.94007302 & -1.64505841 & 2.51601491 \\ \mathrm{H} & -1.26549024 & -1.42126200 & 1.67371590 \\ \mathrm{C} & -0.33904440 & 1.13701656 & -0.46393931 \\ \mathrm{C} & 0.99337757 & 1.74266827 & -0.21912387 \\ \mathrm{C} & 1.61810843 & 1.74804557 & 1.04038468 \\ \mathrm{C} & 1.67623932 & 2.27716940 & -1.32860007 \\ \mathrm{C} & 2.90229330 & 2.27480637 & 1.18454521 \\ \mathrm{H} & 1.12197748 & 1.33169775 & 1.91608435 \\ \mathrm{C} & 2.95357477 & 2.80814698 & -1.18105908 \\ \mathrm{H} & 1.18389841 & 2.25666125 & -2.30164825 \\ \mathrm{C} & 3.57037853 & 2.80511381 & 0.07726699 \\ \mathrm{H} & 3.38296869 & 2.26833688 & 2.16473639 \\ \mathrm{H} & 3.47664956 & 3.22166463 & -2.04590706 \\ \mathrm{H} & 3.21663280 & 0.19312465 \\ & & 1.05961800 & -1.57771740 \\ \mathrm{H} & 0.49931812 & 0.71420748 \\ \mathrm{H} & & & \\ \mathrm{H} & -.91605883 & 1.68912498\end{array}$

\section{Structure 7. eosin Y}

$\mathrm{C}$
$\mathrm{C}$
$\mathrm{C}$
$\mathrm{C}$
$\mathrm{C}$
$-0.20086244$

$-0.02041317$

0.21328144

0.26480278

0.08557720
3.11870080

2.61730905

3.52359685

4.89974683

5.38553296
$-1.73010377$

$-0.43248602$

0.62710012

0.35478792

$-0.93785703$ 


$\begin{array}{lrrr}\mathrm{C} & -0.15012796 & 4.49037951 & -1.98411129 \\ \mathrm{H} & -0.37882603 & 2.41814366 & -2.54803725 \\ \mathrm{H} & 0.44465169 & 5.57876780 & 1.18888216 \\ \mathrm{H} & 0.12717355 & 6.45950946 & -1.12887827 \\ \mathrm{H} & -0.29450343 & 4.85664240 & -3.00262106 \\ \mathrm{C} & 0.40222560 & 3.12955756 & 2.05771211 \\ \mathrm{O} & 0.67868216 & 3.91344359 & 2.93902969 \\ \mathrm{O} & 0.22967430 & 1.82068237 & 2.28650816 \\ \mathrm{H} & 0.37951671 & 1.65731649 & 3.23454942 \\ \mathrm{C} & -0.05809769 & 1.13694737 & -0.26949329 \\ \mathrm{C} & 1.17506386 & 0.39921398 & -0.23272232 \\ \mathrm{C} & 2.44485273 & 1.01023327 & -0.29725535 \\ \mathrm{C} & 1.11276900 & -1.00862292 & -0.12131908 \\ \mathrm{C} & 3.59067168 & 0.24228178 & -0.24505501 \\ \mathrm{H} & 2.51712301 & 2.09299643 & -0.38844572 \\ \mathrm{C} & 2.27429633 & -1.78700229 & -0.07164291 \\ \mathrm{C} & 3.53814854 & -1.17299358 & -0.13074942 \\ \mathrm{C} & -1.26074621 & 0.45419086 & -0.21690447 \\ \mathrm{C} & -2.54009456 & 1.11144736 & -0.24140646 \\ \mathrm{C} & -1.25362190 & -0.98672272 & -0.10357728 \\ \mathrm{C} & -3.69216020 & 0.39745173 & -0.17355459 \\ \mathrm{H} & -2.56336944 & 2.19806998 & -0.31409986 \\ \mathrm{C} & -2.41651054 & -1.71080683 & -0.03348189 \\ \mathrm{C} & -3.73862989 & -1.08293366 & -0.06849169 \\ \mathrm{Br} & 2.15474590 & -3.66836273 & 0.07531014 \\ \mathrm{O} & 4.62621904 & -1.93670814 & -0.07972084 \\ \mathrm{H} & 5.42497328 & -1.38004034 & -0.13201773 \\ \mathrm{Br} & 5.30732797 & 1.06350836 & -0.32931994 \\ \mathrm{Br} & -5.37232591 & 1.27792343 & -0.20764784 \\ \mathrm{O} & -4.79109731 & -1.71232855 & -0.01302856 \\ \mathrm{O} & -0.07199841 & -1.64880748 & -0.05963028 \\ \mathrm{Br} & -2.36549440 & -3.59811785 & 0.11905974\end{array}$

\section{Structure 8. Compound 3a}

$\begin{array}{lrrr}\mathrm{C} & 4.20289487 & -1.93347248 & 0.41036243 \\ \mathrm{C} & 3.74372466 & -0.96231623 & 1.31783187 \\ \mathrm{C} & 2.72335862 & -0.07878796 & 0.97250147 \\ \mathrm{C} & 3.60030971 & -1.98658378 & -0.85370754 \\ \mathrm{H} & 4.19589527 & -0.89860333 & 2.31127420 \\ \mathrm{H} & 2.39231190 & 0.66725490 & 1.69960915 \\ \mathrm{H} & 3.93513271 & -2.73344416 & -1.57819492 \\ \mathrm{C} & 5.31547524 & -2.87531894 & 0.79290012 \\ \mathrm{H} & 6.26290032 & -2.32932936 & 0.93684713 \\ \mathrm{H} & 5.09458941 & -3.38788237 & 1.74299238\end{array}$




$\begin{array}{lrrc}\mathrm{H} & 5.48073650 & -3.63980413 & 0.02032116 \\ \mathrm{C} & 2.12936353 & -0.13511401 & -0.29781500 \\ \mathrm{~S} & -1.32351289 & 1.88802493 & 0.08781972 \\ \mathrm{O} & -1.98746522 & 2.05721534 & 1.38265746 \\ \mathrm{O} & -2.10098166 & 2.08159368 & -1.13797769 \\ \mathrm{~N} & 0.06415547 & 2.87673848 & 0.07353909 \\ \mathrm{C} & -0.04680575 & 4.29321542 & 0.39141702 \\ \mathrm{H} & -0.52745409 & 4.42027636 & 1.37120457 \\ \mathrm{H} & 0.97421811 & 4.69325782 & 0.42271507 \\ \mathrm{H} & -0.62536535 & 4.82312049 & -0.37962512 \\ \mathrm{C} & 0.98739256 & 0.78433444 & -0.66500536 \\ \mathrm{C} & 1.23548692 & 2.25326945 & -0.31614115 \\ \mathrm{O} & 2.30324130 & 2.81941810 & -0.39317477 \\ \mathrm{C} & -0.33923483 & 0.34759991 & -0.02711343 \\ \mathrm{H} & -0.19404934 & 0.02493909 & 1.01146757 \\ \mathrm{C} & -1.10101521 & -0.70357696 & -0.85358939 \\ \mathrm{O} & -0.73892804 & -0.92877000 & -1.99209407 \\ \mathrm{C} & -2.24959267 & -1.40277634 & -0.22780121 \\ \mathrm{C} & -2.67492650 & -1.14776010 & 1.08877089 \\ \mathrm{C} & -2.93306255 & -2.36095193 & -1.00135521 \\ \mathrm{C} & -3.76758321 & -1.83751820 & 1.61622246 \\ \mathrm{H} & -2.18031849 & -0.39982205 & 1.70762428 \\ \mathrm{C} & -4.01894800 & -3.04955250 & -0.47019787 \\ \mathrm{H} & -2.59221539 & -2.54728048 & -2.02064031 \\ \mathrm{C} & -4.43764997 & -2.78852767 & 0.84136200 \\ \mathrm{H} & -4.09524556 & -1.63187674 & 2.63715746 \\ \mathrm{H} & -4.54414112 & -3.79170886 & -1.07504900 \\ \mathrm{H} & -5.29018908 & -3.32875723 & 1.25928280 \\ \mathrm{C} & 2.57663966 & -1.10121835 & -1.20441278 \\ \mathrm{H} & 2.11265288 & -1.16779022 & -2.19073837 \\ \mathrm{H} & 0.84576563 & 0.75656235 & -1.75680048 \\ & & & \\ \mathrm{H} & & \end{array}$

\section{Bioactivity study}

\section{Materials and methods}

Human cervical cancer cell line (Hela), adenocarcinomic human alveolar basal epithelial cells (A549), human breast cancer cell line (MCF7) and human epithelial colorectal adenocarcinoma cells(Caco-2) were cultured in Dulbecco's modified Eagle's medium (DMEM) (Sigma Aldrich, MO, USA) and supplemented with 10\% fetal bovine serum (FBS, Hyclone, CA, USA), $2 \mathrm{mM}$ L-glutamine (Gibco, CA, USA). Cells were seeded in 96 well plates overnight, followed by 
compounds treatment for 72 hours. The crystal violet assay was conducted to determine the percentage of cell viability.

\section{IC 50 $_{0}$ Determination:}

Hela cells were seeded at 2000 cells/well, A549 and MCF7 cells were seeded at 2500 cells/well, Caco- 2 cells were seeded at 2800 cells/well with $100 \mathrm{uL}$ medium in 96 well plates. These cells were allowed to attach overnight, followed by treating with selected compound $\mathbf{3 y}$ ranging from $15.625 \mu \mathrm{M}$ to $1 \mathrm{mM}$ at 2-fold serial dilution on the following day. Cell viability was examined after $72 \mathrm{~h}$ compounds treatment using Crystal Violet staining. $\mathrm{IC}_{50}$ was determined using PRISM software. After $72 \mathrm{~h}$, all compounds at $1 \mathrm{mM}$ showed slightly precipitation so we exclude $1 \mathrm{mM}$ data point from the viability curves.

\section{Results}

Biological activity of isothiazolidinone heterocycles compounds were initially evaluated in human breast cancer cell line (MCF7) and cervical cancer cell line (HeLa) (Figure S10). Several of the analogues, including $\mathbf{3 w}, \mathbf{3 y}, \mathbf{5 n}$ and $\mathbf{5 q}$ were found to reduce the cell viability more than $50 \%$ in both cell lines compared with a DMSO control.
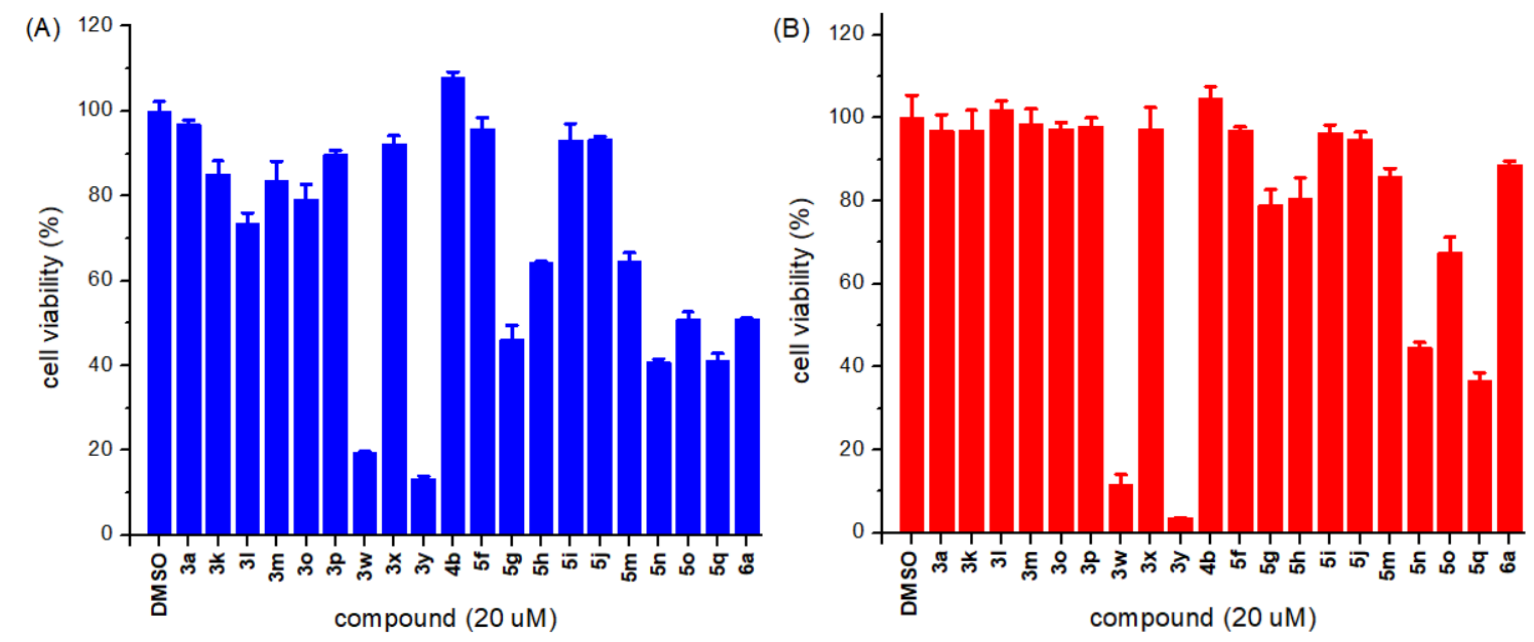

Figure S10. Relative activity of compounds at $20 \mu \mathrm{M}$ in (A) MCF7 and (B) HeLa.

$\mathrm{IC}_{50}$ values of compound $\mathbf{3 y}$ in different cell lines are summarized in Table S2. The cell viability inhibition curve for compound $\mathbf{3 y}$ in 4 cell lines is shown in Figure S11.

Table S2. Summary of IC $\mathbf{5 0}$ values of compound $\mathbf{3 y}$

\begin{tabular}{|c|c|c|c|c|c|c|}
\hline Compound & $\begin{array}{c}\text { Concentration } \\
(\mu \mathrm{M})\end{array}$ & $\begin{array}{c}\text { Treatment } \\
\text { time }\end{array}$ & $\begin{array}{c}\mathrm{IC}_{50}(\mu \mathrm{M}) \\
\text { Hela }\end{array}$ & $\begin{array}{c}\mathrm{IC}_{50}(\mu \mathrm{M}) \\
\mathrm{A} 549\end{array}$ & $\begin{array}{c}\mathrm{IC}_{50}(\mu \mathrm{M}) \\
\mathrm{MCF} 7\end{array}$ & $\begin{array}{c}\mathrm{IC}_{50}(\mu \mathrm{M}) \\
\text { Caco-2 }\end{array}$ \\
\hline $\mathbf{3 y}$ & $0 \sim 500$ & $72 \mathrm{~h}$ & 10.08 & 20.62 & 7.18 & 32.18 \\
\hline
\end{tabular}




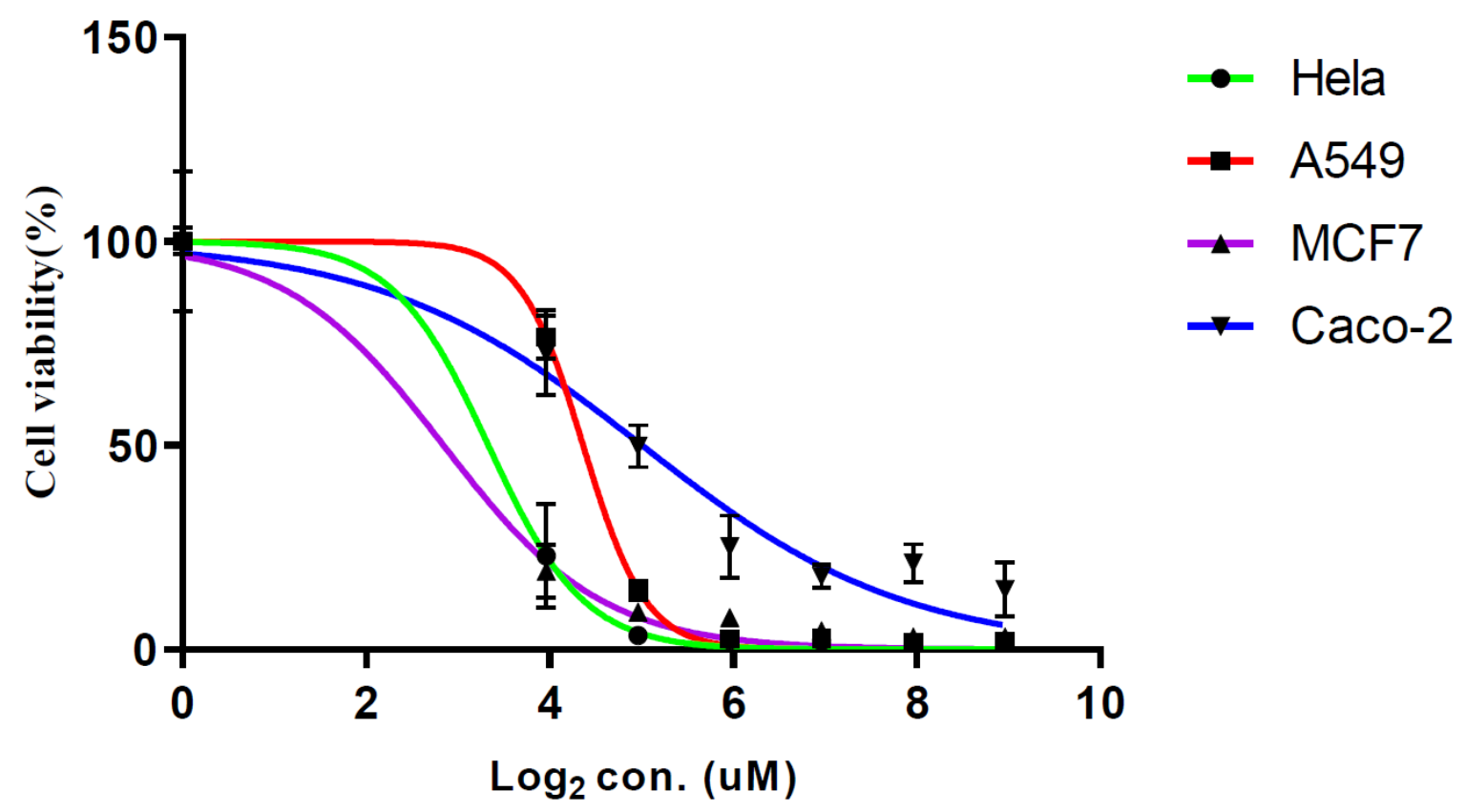

Figure S11. Cell viability inhibition curve for compound $\mathbf{3 y}$ in 4 cell lines. 


\section{Crystal data and structure refinement for 3a}

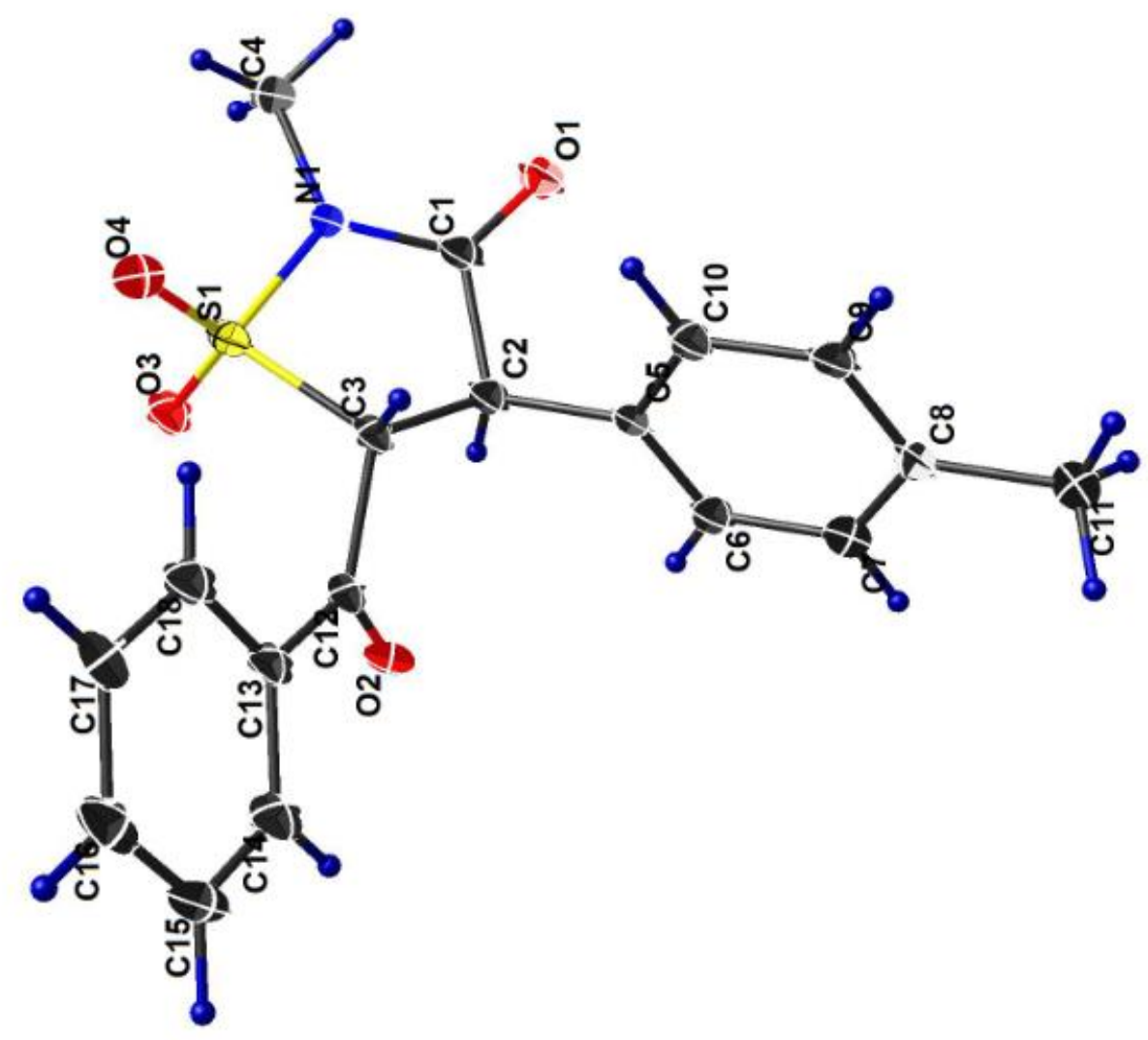

Table S3. Crystal data and structure refinement for 3a.

Identification code

Empirical formula

Formula weight

Temperature

Wavelength

Crystal system

Space group

Unit cell dimensions

Volume

Z

Density (calculated)
I644 (3a)

C18 H17 N O4 S

343.38

$100(2) \mathrm{K}$

$1.54178 \AA$

Triclinic

P-1

$\mathrm{a}=5.8230(2) \AA$ $\alpha=99.7650(10)^{\circ}$.

$\mathrm{b}=7.3298(3) \AA$

$\beta=92.9640(10)^{\circ}$.

$c=19.6198(7) \AA$ $\gamma=104.8250(10)^{\circ}$.
2

$1.437 \mathrm{Mg} / \mathrm{m}^{3}$ 
Absorption coefficient

$\mathrm{F}(000)$

Crystal size

Theta range for data collection

Index ranges

Reflections collected

Independent reflections

Completeness to theta $=66.598^{\circ}$

Absorption correction

Max. and min. transmission

Refinement method

Data / restraints / parameters

Goodness-of-fit on $\mathrm{F}^{2}$

Final $R$ indices [I $>2 \operatorname{sigma}(\mathrm{I})]$

$\mathrm{R}$ indices (all data)

Extinction coefficient

Largest diff. peak and hole
$2.013 \mathrm{~mm}^{-1}$

360

$0.545 \times 0.351 \times 0.159 \mathrm{~mm}^{3}$

2.296 to $66.598^{\circ}$.

$-6<=\mathrm{h}<=6,-8<=\mathrm{k}<=8,-23<=\mathrm{l}<=23$

4289

$4289[\mathrm{R}(\mathrm{int})=$ ?]

$98.3 \%$

Semi-empirical from equivalents

0.752820 and 0.284725

Full-matrix least-squares on $\mathrm{F}^{2}$

4289 / 0 / 220

1.058

$\mathrm{R} 1=0.0833, \mathrm{wR} 2=0.2298$

$\mathrm{R} 1=0.0860, \mathrm{wR} 2=0.2377$

$\mathrm{n} / \mathrm{a}$

0.826 and -0.979 e. $\AA^{-3}$

Table S4. Atomic coordinates (x 104) and equivalent isotropic displacement parameters $\left(\AA^{2} \mathrm{x}\right.$ $10^{3}$ )for 3a. U(eq) is defined as one third of the trace of the orthogonalized $U^{i j}$ tensor.

\begin{tabular}{lrrrr}
\hline & $\mathrm{x}$ & $\mathrm{y}$ & $\mathrm{z}$ & $\mathrm{U}(\mathrm{eq})$ \\
\hline $\mathrm{S}(1)$ & $4822(1)$ & $1077(1)$ & $3151(1)$ & $19(1)$ \\
$\mathrm{O}(1)$ & $2193(5)$ & $404(4)$ & $1326(1)$ & $28(1)$ \\
$\mathrm{O}(2)$ & $10457(4)$ & $3462(4)$ & $2711(1)$ & $24(1)$ \\
$\mathrm{O}(3)$ & $6388(5)$ & $-108(4)$ & $3234(1)$ & $27(1)$ \\
$\mathrm{O}(4)$ & $3561(5)$ & $1592(4)$ & $3727(1)$ & $27(1)$ \\
$\mathrm{N}(1)$ & $2894(5)$ & $130(4)$ & $2456(2)$ & $20(1)$ \\
$\mathrm{C}(1)$ & $3471(6)$ & $896(5)$ & $1868(2)$ & $21(1)$ \\
$\mathrm{C}(2)$ & $5921(6)$ & $2346(5)$ & $1999(2)$ & $20(1)$ \\
$\mathrm{C}(3)$ & $6309(6)$ & $3110(5)$ & $2789(2)$ & $18(1)$ \\
& & 557 & &
\end{tabular}




\begin{tabular}{lrrrr}
$\mathrm{C}(4)$ & $702(7)$ & $-1384(6)$ & $2475(2)$ & $27(1)$ \\
$\mathrm{C}(5)$ & $6181(6)$ & $3918(5)$ & $1575(2)$ & $20(1)$ \\
$\mathrm{C}(6)$ & $8004(6)$ & $4260(5)$ & $1147(2)$ & $22(1)$ \\
$\mathrm{C}(7)$ & $8209(7)$ & $5701(6)$ & $756(2)$ & $24(1)$ \\
$\mathrm{C}(8)$ & $6583(7)$ & $6808(5)$ & $776(2)$ & $23(1)$ \\
$\mathrm{C}(9)$ & $4749(6)$ & $6447(5)$ & $1206(2)$ & $22(1)$ \\
$\mathrm{C}(10)$ & $4548(7)$ & $5034(6)$ & $1607(2)$ & $22(1)$ \\
$\mathrm{C}(11)$ & $6813(7)$ & $8349(6)$ & $344(2)$ & $29(1)$ \\
$\mathrm{C}(12)$ & $8973(6)$ & $3924(5)$ & $3066(2)$ & $20(1)$ \\
$\mathrm{C}(13)$ & $9627(6)$ & $5320(5)$ & $3736(2)$ & $21(1)$ \\
$\mathrm{C}(14)$ & $12006(7)$ & $6382(6)$ & $3895(2)$ & $25(1)$ \\
$\mathrm{C}(15)$ & $12712(7)$ & $7687(6)$ & $4515(2)$ & $28(1)$ \\
$\mathrm{C}(16)$ & $11048(7)$ & $7936(6)$ & $4984(2)$ & $26(1)$ \\
$\mathrm{C}(17)$ & $8689(7)$ & $6897(6)$ & $4827(2)$ & $26(1)$ \\
$\mathrm{C}(18)$ & $7951(7)$ & $5592(6)$ & $4195(2)$ & $23(1)$ \\
\hline
\end{tabular}

Table S5. Bond lengths $[\AA ̊]$ and angles $\left[{ }^{\circ}\right]$ for $\mathbf{3 a}$.

\begin{tabular}{ll}
\hline $\mathrm{S}(1)-\mathrm{O}(4)$ & $1.429(3)$ \\
$\mathrm{S}(1)-\mathrm{O}(3)$ & $1.432(3)$ \\
$\mathrm{S}(1)-\mathrm{N}(1)$ & $1.651(3)$ \\
$\mathrm{S}(1)-\mathrm{C}(3)$ & $1.800(4)$ \\
$\mathrm{O}(1)-\mathrm{C}(1)$ & $1.210(4)$ \\
$\mathrm{O}(2)-\mathrm{C}(12)$ & $1.215(4)$ \\
$\mathrm{N}(1)-\mathrm{C}(1)$ & $1.387(5)$ \\
$\mathrm{N}(1)-\mathrm{C}(4)$ & $1.468(5)$ \\
$\mathrm{C}(1)-\mathrm{C}(2)$ & $1.525(5)$ \\
$\mathrm{C}(2)-\mathrm{C}(5)$ & $1.514(5)$ \\
$\mathrm{C}(2)-\mathrm{C}(3)$ & $1.539(4)$ \\
$\mathrm{C}(2)-\mathrm{H}(2)$ & 1.0000 \\
$\mathrm{C}(3)-\mathrm{C}(12)$ & $1.544(5)$ \\
$\mathrm{C}(3)-\mathrm{H}(3)$ & 1.0000 \\
$\mathrm{C}(4)-\mathrm{H}(4 \mathrm{~A})$ & 0.9800 \\
$\mathrm{C}(4)-\mathrm{H}(4 \mathrm{~B})$ & 0.9800 \\
$\mathrm{C}(4)-\mathrm{H}(4 \mathrm{C})$ & 0.9800 \\
$\mathrm{C}(5)-\mathrm{C}(6)$ & $1.387(5)$ \\
$\mathrm{C}(5)-\mathrm{C}(10)$ & $1.401(5)$
\end{tabular}




\begin{tabular}{|c|c|}
\hline$C(6)-C(7)$ & $1.392(5)$ \\
\hline $\mathrm{C}(6)-\mathrm{H}(6)$ & 0.9500 \\
\hline$C(7)-C(8)$ & $1.394(6)$ \\
\hline $\mathrm{C}(7)-\mathrm{H}(7)$ & 0.9500 \\
\hline $\mathrm{C}(8)-\mathrm{C}(9)$ & $1.395(5)$ \\
\hline$C(8)-C(11)$ & $1.505(5)$ \\
\hline $\mathrm{C}(9)-\mathrm{C}(10)$ & $1.391(5)$ \\
\hline $\mathrm{C}(9)-\mathrm{H}(9)$ & 0.9500 \\
\hline $\mathrm{C}(10)-\mathrm{H}(10)$ & 0.9500 \\
\hline $\mathrm{C}(11)-\mathrm{H}(11 \mathrm{~A})$ & 0.9800 \\
\hline $\mathrm{C}(11)-\mathrm{H}(11 \mathrm{~B})$ & 0.9800 \\
\hline $\mathrm{C}(11)-\mathrm{H}(11 \mathrm{C})$ & 0.9800 \\
\hline$C(12)-C(13)$ & $1.489(5)$ \\
\hline $\mathrm{C}(13)-\mathrm{C}(18)$ & $1.390(5)$ \\
\hline$C(13)-C(14)$ & $1.394(5)$ \\
\hline$C(14)-C(15)$ & $1.384(6)$ \\
\hline $\mathrm{C}(14)-\mathrm{H}(14)$ & 0.9500 \\
\hline$C(15)-C(16)$ & $1.394(6)$ \\
\hline $\mathrm{C}(15)-\mathrm{H}(15)$ & 0.9500 \\
\hline$C(16)-C(17)$ & $1.380(6)$ \\
\hline $\mathrm{C}(16)-\mathrm{H}(16)$ & 0.9500 \\
\hline $\mathrm{C}(17)-\mathrm{C}(18)$ & $1.404(6)$ \\
\hline $\mathrm{C}(17)-\mathrm{H}(17)$ & 0.9500 \\
\hline C(18)-H(18) & 0.9500 \\
\hline $\mathrm{O}(4)-\mathrm{S}(1)-\mathrm{O}(3)$ & $117.80(16)$ \\
\hline $\mathrm{O}(4)-\mathrm{S}(1)-\mathrm{N}(1)$ & $108.99(15)$ \\
\hline $\mathrm{O}(3)-\mathrm{S}(1)-\mathrm{N}(1)$ & $110.79(16)$ \\
\hline $\mathrm{O}(4)-\mathrm{S}(1)-\mathrm{C}(3)$ & $112.84(16)$ \\
\hline $\mathrm{O}(3)-\mathrm{S}(1)-\mathrm{C}(3)$ & $110.35(16)$ \\
\hline $\mathrm{N}(1)-\mathrm{S}(1)-\mathrm{C}(3)$ & $93.36(15)$ \\
\hline $\mathrm{C}(1)-\mathrm{N}(1)-\mathrm{C}(4)$ & $123.2(3)$ \\
\hline $\mathrm{C}(1)-\mathrm{N}(1)-\mathrm{S}(1)$ & $115.3(2)$ \\
\hline $\mathrm{C}(4)-\mathrm{N}(1)-\mathrm{S}(1)$ & $121.5(2)$ \\
\hline $\mathrm{O}(1)-\mathrm{C}(1)-\mathrm{N}(1)$ & $123.1(3)$ \\
\hline $\mathrm{O}(1)-\mathrm{C}(1)-\mathrm{C}(2)$ & $126.2(3)$ \\
\hline $\mathrm{N}(1)-\mathrm{C}(1)-\mathrm{C}(2)$ & $110.6(3)$ \\
\hline$C(5)-C(2)-C(1)$ & $113.6(3)$ \\
\hline
\end{tabular}




$\begin{array}{ll}\mathrm{C}(5)-\mathrm{C}(2)-\mathrm{C}(3) & 113.4(3) \\ \mathrm{C}(1)-\mathrm{C}(2)-\mathrm{C}(3) & 105.6(3) \\ \mathrm{C}(5)-\mathrm{C}(2)-\mathrm{H}(2) & 108.0 \\ \mathrm{C}(1)-\mathrm{C}(2)-\mathrm{H}(2) & 108.0 \\ \mathrm{C}(3)-\mathrm{C}(2)-\mathrm{H}(2) & 108.0 \\ \mathrm{C}(2)-\mathrm{C}(3)-\mathrm{C}(12) & 113.0(3) \\ \mathrm{C}(2)-\mathrm{C}(3)-\mathrm{S}(1) & 103.9(2) \\ \mathrm{C}(12)-\mathrm{C}(3)-\mathrm{S}(1) & 113.1(2) \\ \mathrm{C}(2)-\mathrm{C}(3)-\mathrm{H}(3) & 108.9 \\ \mathrm{C}(12)-\mathrm{C}(3)-\mathrm{H}(3) & 108.9 \\ \mathrm{~S}(1)-\mathrm{C}(3)-\mathrm{H}(3) & 108.9 \\ \mathrm{~N}(1)-\mathrm{C}(4)-\mathrm{H}(4 \mathrm{~A}) & 109.5 \\ \mathrm{~N}(1)-\mathrm{C}(4)-\mathrm{H}(4 \mathrm{~B}) & 109.5 \\ \mathrm{H}(4 \mathrm{~A})-\mathrm{C}(4)-\mathrm{H}(4 \mathrm{~B}) & 109.5 \\ \mathrm{~N}(1)-\mathrm{C}(4)-\mathrm{H}(4 \mathrm{C}) & 109.5 \\ \mathrm{H}(4 \mathrm{~A})-\mathrm{C}(4)-\mathrm{H}(4 \mathrm{C}) & 109.5 \\ \mathrm{H}(4 \mathrm{~B})-\mathrm{C}(4)-\mathrm{H}(4 \mathrm{C}) & 109.5 \\ \mathrm{C}(6)-\mathrm{C}(5)-\mathrm{C}(10) & 119.3(3) \\ \mathrm{C}(6)-\mathrm{C}(5)-\mathrm{C}(2) & 120.9(3) \\ \mathrm{C}(10)-\mathrm{C}(5)-\mathrm{C}(2) & 119.8(3) \\ \mathrm{C}(5)-\mathrm{C}(6)-\mathrm{C}(7) & 120.2(3) \\ \mathrm{C}(5)-\mathrm{C}(6)-\mathrm{H}(6) & 119.9 \\ \mathrm{C}(7)-\mathrm{C}(6)-\mathrm{H}(6) & 119.9 \\ \mathrm{C}(6)-\mathrm{C}(7)-\mathrm{C}(8) & 121.3(3) \\ \mathrm{C}(6)-\mathrm{C}(7)-\mathrm{H}(7) & 119.3 \\ \mathrm{C}(8)-\mathrm{C}(7)-\mathrm{H}(7) & 119.3 \\ \mathrm{C}(7)-\mathrm{C}(8)-\mathrm{C}(9) & 118.1(3) \\ \mathrm{C}(7)-\mathrm{C}(8)-\mathrm{C}(11) & 120.7(3) \\ \mathrm{C}(9)-\mathrm{C}(8)-\mathrm{C}(11) & 121.3(3) \\ \mathrm{C}(10)-\mathrm{C}(9)-\mathrm{C}(8) & 121.2(3) \\ \mathrm{C}(10)-\mathrm{C}(9)-\mathrm{H}(9) & 119.4 \\ \mathrm{C}(8)-\mathrm{C}(9)-\mathrm{H}(9) & 119.4 \\ \mathrm{C}(9)-\mathrm{C}(10)-\mathrm{C}(5) & 119.9(3) \\ \mathrm{C}(9)-\mathrm{C}(10)-\mathrm{H}(10) & 120.0 \\ \mathrm{C}(5)-\mathrm{C}(10)-\mathrm{H}(10) & 120.0 \\ \mathrm{C}(8)-\mathrm{C}(11)-\mathrm{H}(11 \mathrm{~A}) & 109.5 \\ \mathrm{C}(8)-\mathrm{C}(11)-\mathrm{H}(11 \mathrm{~B}) & 109.5 \\ \mathrm{H}(11 \mathrm{~A})-\mathrm{C}(11)-\mathrm{H}(11 \mathrm{~B}) & 109.5 \\ & \end{array}$




$\begin{array}{ll}\mathrm{C}(8)-\mathrm{C}(11)-\mathrm{H}(11 \mathrm{C}) & 109.5 \\ \mathrm{H}(11 \mathrm{~A})-\mathrm{C}(11)-\mathrm{H}(11 \mathrm{C}) & 109.5 \\ \mathrm{H}(11 \mathrm{~B})-\mathrm{C}(11)-\mathrm{H}(11 \mathrm{C}) & 109.5 \\ \mathrm{O}(2)-\mathrm{C}(12)-\mathrm{C}(13) & 122.6(3) \\ \mathrm{O}(2)-\mathrm{C}(12)-\mathrm{C}(3) & 118.5(3) \\ \mathrm{C}(13)-\mathrm{C}(12)-\mathrm{C}(3) & 118.8(3) \\ \mathrm{C}(18)-\mathrm{C}(13)-\mathrm{C}(14) & 120.1(3) \\ \mathrm{C}(18)-\mathrm{C}(13)-\mathrm{C}(12) & 122.1(3) \\ \mathrm{C}(14)-\mathrm{C}(13)-\mathrm{C}(12) & 117.8(3) \\ \mathrm{C}(15)-\mathrm{C}(14)-\mathrm{C}(13) & 120.1(4) \\ \mathrm{C}(15)-\mathrm{C}(14)-\mathrm{H}(14) & 120.0 \\ \mathrm{C}(13)-\mathrm{C}(14)-\mathrm{H}(14) & 120.0 \\ \mathrm{C}(14)-\mathrm{C}(15)-\mathrm{C}(16) & 120.1(4) \\ \mathrm{C}(14)-\mathrm{C}(15)-\mathrm{H}(15) & 120.0 \\ \mathrm{C}(16)-\mathrm{C}(15)-\mathrm{H}(15) & 120.0 \\ \mathrm{C}(17)-\mathrm{C}(16)-\mathrm{C}(15) & 120.1(3) \\ \mathrm{C}(17)-\mathrm{C}(16)-\mathrm{H}(16) & 120.0 \\ \mathrm{C}(15)-\mathrm{C}(16)-\mathrm{H}(16) & 120.0 \\ \mathrm{C}(16)-\mathrm{C}(17)-\mathrm{C}(18) & 120.2(4) \\ \mathrm{C}(16)-\mathrm{C}(17)-\mathrm{H}(17) & 119.9 \\ \mathrm{C}(18)-\mathrm{C}(17)-\mathrm{H}(17) & 119.9 \\ \mathrm{C}(13)-\mathrm{C}(18)-\mathrm{C}(17) & 119.5(4) \\ \mathrm{C}(13)-\mathrm{C}(18)-\mathrm{H}(18) & 120.3 \\ \mathrm{C}(17)-\mathrm{C}(18)-\mathrm{H}(18) & 120.3 \\ & \end{array}$

Symmetry transformations used to generate equivalent atoms: 
Table S6. Anisotropic displacement parameters $\left(\AA^{2} \times 10^{3}\right)$ for $3 \mathbf{a}$. The anisotropic displacement factor exponent takes the form: $-2 \mathrm{p}^{2}\left[\mathrm{~h}^{2} \mathrm{a}^{* 2} \mathrm{U}^{11}+\ldots+2 \mathrm{~h} \mathrm{k} \mathrm{a}^{*} \mathrm{~b}^{*} \mathrm{U}^{12}\right]$

\begin{tabular}{|c|c|c|c|c|c|c|}
\hline & $\mathrm{U}^{11}$ & $\mathrm{U}^{22}$ & $\mathrm{U}^{33}$ & $\mathrm{U}^{23}$ & $\mathrm{U}^{13}$ & $\mathrm{U}^{12}$ \\
\hline$S(1)$ & $11(1)$ & $23(1)$ & $24(1)$ & $7(1)$ & $-3(1)$ & $3(1)$ \\
\hline $\mathrm{O}(1)$ & $22(1)$ & $27(2)$ & $29(1)$ & $6(1)$ & $-9(1)$ & $1(1)$ \\
\hline $\mathrm{O}(2)$ & $14(1)$ & $31(2)$ & $28(1)$ & $7(1)$ & $-1(1)$ & $8(1)$ \\
\hline $\mathrm{O}(3)$ & $21(1)$ & $27(2)$ & $34(1)$ & $13(1)$ & $-4(1)$ & $7(1)$ \\
\hline $\mathrm{O}(4)$ & $20(1)$ & $31(2)$ & $28(1)$ & $6(1)$ & $2(1)$ & $2(1)$ \\
\hline $\mathrm{N}(1)$ & $13(2)$ & $21(2)$ & $25(1)$ & $5(1)$ & $-4(1)$ & $3(1)$ \\
\hline $\mathrm{C}(1)$ & $15(2)$ & $22(2)$ & $27(2)$ & $6(1)$ & $-4(1)$ & $6(2)$ \\
\hline $\mathrm{C}(2)$ & $15(2)$ & $22(2)$ & $23(2)$ & $5(1)$ & $-3(1)$ & $7(1)$ \\
\hline $\mathrm{C}(3)$ & $15(2)$ & $20(2)$ & $22(2)$ & $6(1)$ & $-2(1)$ & $7(1)$ \\
\hline $\mathrm{C}(4)$ & $16(2)$ & $30(2)$ & $33(2)$ & $9(2)$ & $-2(1)$ & $3(2)$ \\
\hline$C(5)$ & $13(2)$ & $22(2)$ & $23(2)$ & $5(1)$ & $-6(1)$ & $4(1)$ \\
\hline$C(6)$ & $16(2)$ & $24(2)$ & $25(2)$ & $5(1)$ & $-2(1)$ & $6(1)$ \\
\hline$C(7)$ & $18(2)$ & $30(2)$ & $24(2)$ & $7(1)$ & $-2(1)$ & $3(2)$ \\
\hline$C(8)$ & $20(2)$ & $22(2)$ & $24(2)$ & $6(1)$ & $-9(1)$ & $1(2)$ \\
\hline $\mathrm{C}(9)$ & $12(2)$ & $23(2)$ & $32(2)$ & $7(1)$ & $-4(1)$ & $6(1)$ \\
\hline$C(10)$ & $15(2)$ & $24(2)$ & $28(2)$ & $8(1)$ & $-2(1)$ & $7(1)$ \\
\hline $\mathrm{C}(11)$ & $25(2)$ & $32(2)$ & $31(2)$ & $14(2)$ & $-6(2)$ & $3(2)$ \\
\hline$C(12)$ & $15(2)$ & $20(2)$ & $28(2)$ & $12(1)$ & $-2(1)$ & $6(1)$ \\
\hline $\mathrm{C}(13)$ & $17(2)$ & $21(2)$ & $25(2)$ & $8(1)$ & $-5(1)$ & $7(1)$ \\
\hline$C(14)$ & $19(2)$ & $26(2)$ & $33(2)$ & $10(2)$ & $-3(1)$ & $10(2)$ \\
\hline$C(15)$ & $19(2)$ & $23(2)$ & $38(2)$ & $4(2)$ & $-12(2)$ & $2(2)$ \\
\hline$C(16)$ & $29(2)$ & $22(2)$ & $28(2)$ & $5(2)$ & $-8(2)$ & $9(2)$ \\
\hline$C(17)$ & $27(2)$ & $27(2)$ & $27(2)$ & $7(1)$ & $-1(1)$ & $12(2)$ \\
\hline $\mathrm{C}(18)$ & $18(2)$ & $24(2)$ & $29(2)$ & $8(1)$ & $-3(1)$ & $7(2)$ \\
\hline
\end{tabular}


Table S7. Hydrogen coordinates (x 104) and isotropic displacement parameters $\left(\AA^{2} \times 10^{3}\right)$ for $3 a$.

\begin{tabular}{|c|c|c|c|c|}
\hline & $\mathrm{x}$ & $\mathrm{y}$ & $\mathrm{z}$ & $\mathrm{U}(\mathrm{eq})$ \\
\hline $\mathrm{H}(2)$ & 7143 & 1638 & 1874 & 24 \\
\hline $\mathrm{H}(3)$ & 5481 & 4147 & 2901 & 22 \\
\hline $\mathrm{H}(4 \mathrm{~A})$ & -476 & -1408 & 2096 & 40 \\
\hline $\mathrm{H}(4 \mathrm{~B})$ & 54 & -1122 & 2921 & 40 \\
\hline $\mathrm{H}(4 \mathrm{C})$ & 1066 & -2631 & 2420 & 40 \\
\hline $\mathrm{H}(6)$ & 9116 & 3508 & 1119 & 26 \\
\hline $\mathrm{H}(7)$ & 9482 & 5936 & 471 & 29 \\
\hline $\mathrm{H}(9)$ & 3617 & 7181 & 1224 & 27 \\
\hline $\mathrm{H}(10)$ & 3305 & 4824 & 1904 & 26 \\
\hline $\mathrm{H}(11 \mathrm{~A})$ & 8390 & 9270 & 462 & 44 \\
\hline $\mathrm{H}(11 \mathrm{~B})$ & 5573 & 9017 & 440 & 44 \\
\hline $\mathrm{H}(11 \mathrm{C})$ & 6625 & 7762 & -150 & 44 \\
\hline $\mathrm{H}(14)$ & 13144 & 6210 & 3577 & 30 \\
\hline $\mathrm{H}(15)$ & 14332 & 8413 & 4622 & 34 \\
\hline $\mathrm{H}(16)$ & 11539 & 8821 & 5411 & 32 \\
\hline $\mathrm{H}(17)$ & 7559 & 7065 & 5148 & 31 \\
\hline $\mathrm{H}(18)$ & 6320 & 4900 & 4081 & 28 \\
\hline
\end{tabular}


Table S8. Torsion angles $\left[{ }^{\circ}\right]$ for $\mathbf{3 a}$.

\begin{tabular}{|c|c|}
\hline $\mathrm{O}(4)-\mathrm{S}(1)-\mathrm{N}(1)-\mathrm{C}(1)$ & $129.9(3)$ \\
\hline $\mathrm{O}(3)-\mathrm{S}(1)-\mathrm{N}(1)-\mathrm{C}(1)$ & $-98.9(3)$ \\
\hline $\mathrm{C}(3)-\mathrm{S}(1)-\mathrm{N}(1)-\mathrm{C}(1)$ & $14.3(3)$ \\
\hline $\mathrm{O}(4)-\mathrm{S}(1)-\mathrm{N}(1)-\mathrm{C}(4)$ & $-48.6(3)$ \\
\hline $\mathrm{O}(3)-\mathrm{S}(1)-\mathrm{N}(1)-\mathrm{C}(4)$ & $82.6(3)$ \\
\hline $\mathrm{C}(3)-\mathrm{S}(1)-\mathrm{N}(1)-\mathrm{C}(4)$ & $-164.2(3)$ \\
\hline $\mathrm{C}(4)-\mathrm{N}(1)-\mathrm{C}(1)-\mathrm{O}(1)$ & $1.4(6)$ \\
\hline $\mathrm{S}(1)-\mathrm{N}(1)-\mathrm{C}(1)-\mathrm{O}(1)$ & $-177.1(3)$ \\
\hline $\mathrm{C}(4)-\mathrm{N}(1)-\mathrm{C}(1)-\mathrm{C}(2)$ & $-176.5(3)$ \\
\hline $\mathrm{S}(1)-\mathrm{N}(1)-\mathrm{C}(1)-\mathrm{C}(2)$ & $5.0(4)$ \\
\hline $\mathrm{O}(1)-\mathrm{C}(1)-\mathrm{C}(2)-\mathrm{C}(5)$ & $31.5(5)$ \\
\hline $\mathrm{N}(1)-\mathrm{C}(1)-\mathrm{C}(2)-\mathrm{C}(5)$ & $-150.7(3)$ \\
\hline $\mathrm{O}(1)-\mathrm{C}(1)-\mathrm{C}(2)-\mathrm{C}(3)$ & $156.3(4)$ \\
\hline $\mathrm{N}(1)-\mathrm{C}(1)-\mathrm{C}(2)-\mathrm{C}(3)$ & $-25.8(4)$ \\
\hline$C(5)-C(2)-C(3)-C(12)$ & $-78.5(4)$ \\
\hline $\mathrm{C}(1)-\mathrm{C}(2)-\mathrm{C}(3)-\mathrm{C}(12)$ & $156.6(3)$ \\
\hline$C(5)-C(2)-C(3)-S(1)$ & $158.5(2)$ \\
\hline $\mathrm{C}(1)-\mathrm{C}(2)-\mathrm{C}(3)-\mathrm{S}(1)$ & $33.6(3)$ \\
\hline $\mathrm{O}(4)-\mathrm{S}(1)-\mathrm{C}(3)-\mathrm{C}(2)$ & $-140.3(2)$ \\
\hline $\mathrm{O}(3)-\mathrm{S}(1)-\mathrm{C}(3)-\mathrm{C}(2)$ & $85.6(2)$ \\
\hline $\mathrm{N}(1)-\mathrm{S}(1)-\mathrm{C}(3)-\mathrm{C}(2)$ & $-28.0(2)$ \\
\hline $\mathrm{O}(4)-\mathrm{S}(1)-\mathrm{C}(3)-\mathrm{C}(12)$ & $96.8(3)$ \\
\hline $\mathrm{O}(3)-\mathrm{S}(1)-\mathrm{C}(3)-\mathrm{C}(12)$ & $-37.3(3)$ \\
\hline $\mathrm{N}(1)-\mathrm{S}(1)-\mathrm{C}(3)-\mathrm{C}(12)$ & $-151.0(3)$ \\
\hline$C(1)-C(2)-C(5)-C(6)$ & $-124.3(4)$ \\
\hline$C(3)-C(2)-C(5)-C(6)$ & $115.1(4)$ \\
\hline $\mathrm{C}(1)-\mathrm{C}(2)-\mathrm{C}(5)-\mathrm{C}(10)$ & $55.0(4)$ \\
\hline$C(3)-C(2)-C(5)-C(10)$ & $-65.6(4)$ \\
\hline $\mathrm{C}(10)-\mathrm{C}(5)-\mathrm{C}(6)-\mathrm{C}(7)$ & $0.3(5)$ \\
\hline$C(2)-C(5)-C(6)-C(7)$ & $179.6(3)$ \\
\hline$C(5)-C(6)-C(7)-C(8)$ & $-1.1(5)$ \\
\hline $\mathrm{C}(6)-\mathrm{C}(7)-\mathrm{C}(8)-\mathrm{C}(9)$ & $0.8(5)$ \\
\hline $\mathrm{C}(6)-\mathrm{C}(7)-\mathrm{C}(8)-\mathrm{C}(11)$ & $-179.3(3)$ \\
\hline$C(7)-C(8)-C(9)-C(10)$ & $0.4(5)$ \\
\hline$C(11)-C(8)-C(9)-C(10)$ & $-179.5(3)$ \\
\hline$C(8)-C(9)-C(10)-C(5)$ & $-1.2(6)$ \\
\hline
\end{tabular}




$\begin{array}{lc}\mathrm{C}(6)-\mathrm{C}(5)-\mathrm{C}(10)-\mathrm{C}(9) & 0.8(5) \\ \mathrm{C}(2)-\mathrm{C}(5)-\mathrm{C}(10)-\mathrm{C}(9) & -178.5(3) \\ \mathrm{C}(2)-\mathrm{C}(3)-\mathrm{C}(12)-\mathrm{O}(2) & -18.9(4) \\ \mathrm{S}(1)-\mathrm{C}(3)-\mathrm{C}(12)-\mathrm{O}(2) & 98.8(3) \\ \mathrm{C}(2)-\mathrm{C}(3)-\mathrm{C}(12)-\mathrm{C}(13) & 157.9(3) \\ \mathrm{S}(1)-\mathrm{C}(3)-\mathrm{C}(12)-\mathrm{C}(13) & -84.3(3) \\ \mathrm{O}(2)-\mathrm{C}(12)-\mathrm{C}(13)-\mathrm{C}(18) & -168.6(3) \\ \mathrm{C}(3)-\mathrm{C}(12)-\mathrm{C}(13)-\mathrm{C}(18) & 14.7(5) \\ \mathrm{O}(2)-\mathrm{C}(12)-\mathrm{C}(13)-\mathrm{C}(14) & 11.9(5) \\ \mathrm{C}(3)-\mathrm{C}(12)-\mathrm{C}(13)-\mathrm{C}(14) & -164.8(3) \\ \mathrm{C}(18)-\mathrm{C}(13)-\mathrm{C}(14)-\mathrm{C}(15) & 1.1(5) \\ \mathrm{C}(12)-\mathrm{C}(13)-\mathrm{C}(14)-\mathrm{C}(15) & -179.4(3) \\ \mathrm{C}(13)-\mathrm{C}(14)-\mathrm{C}(15)-\mathrm{C}(16) & 0.3(6) \\ \mathrm{C}(14)-\mathrm{C}(15)-\mathrm{C}(16)-\mathrm{C}(17) & -0.7(5) \\ \mathrm{C}(15)-\mathrm{C}(16)-\mathrm{C}(17)-\mathrm{C}(18) & -0.3(5) \\ \mathrm{C}(14)-\mathrm{C}(13)-\mathrm{C}(18)-\mathrm{C}(17) & -2.0(5) \\ \mathrm{C}(12)-\mathrm{C}(13)-\mathrm{C}(18)-\mathrm{C}(17) & 178.5(3) \\ \mathrm{C}(16)-\mathrm{C}(17)-\mathrm{C}(18)-\mathrm{C}(13) & 1.6(5)\end{array}$

Symmetry transformations used to generate equivalent atoms 


\section{NMR spectra of new compounds}

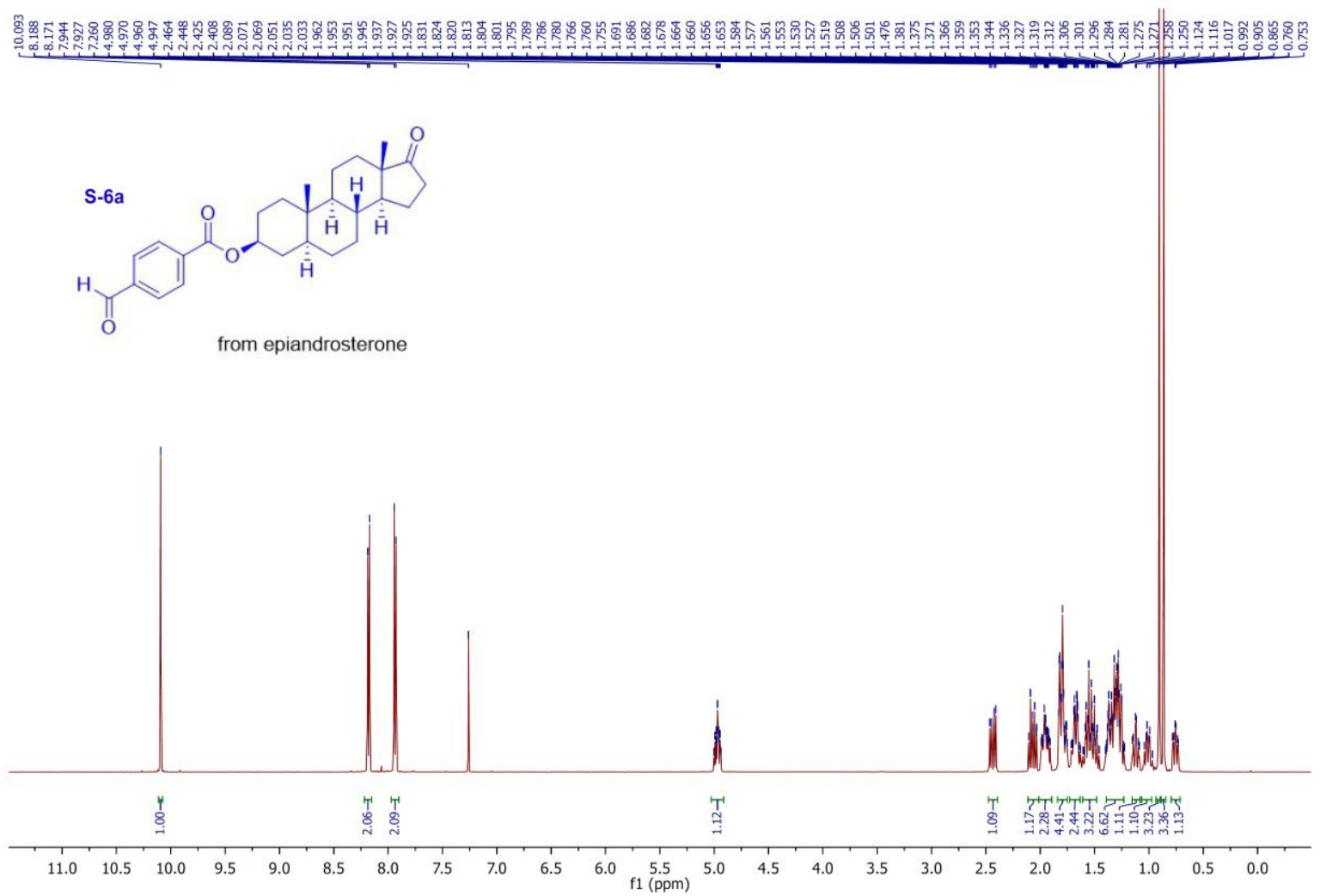

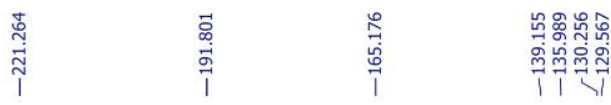
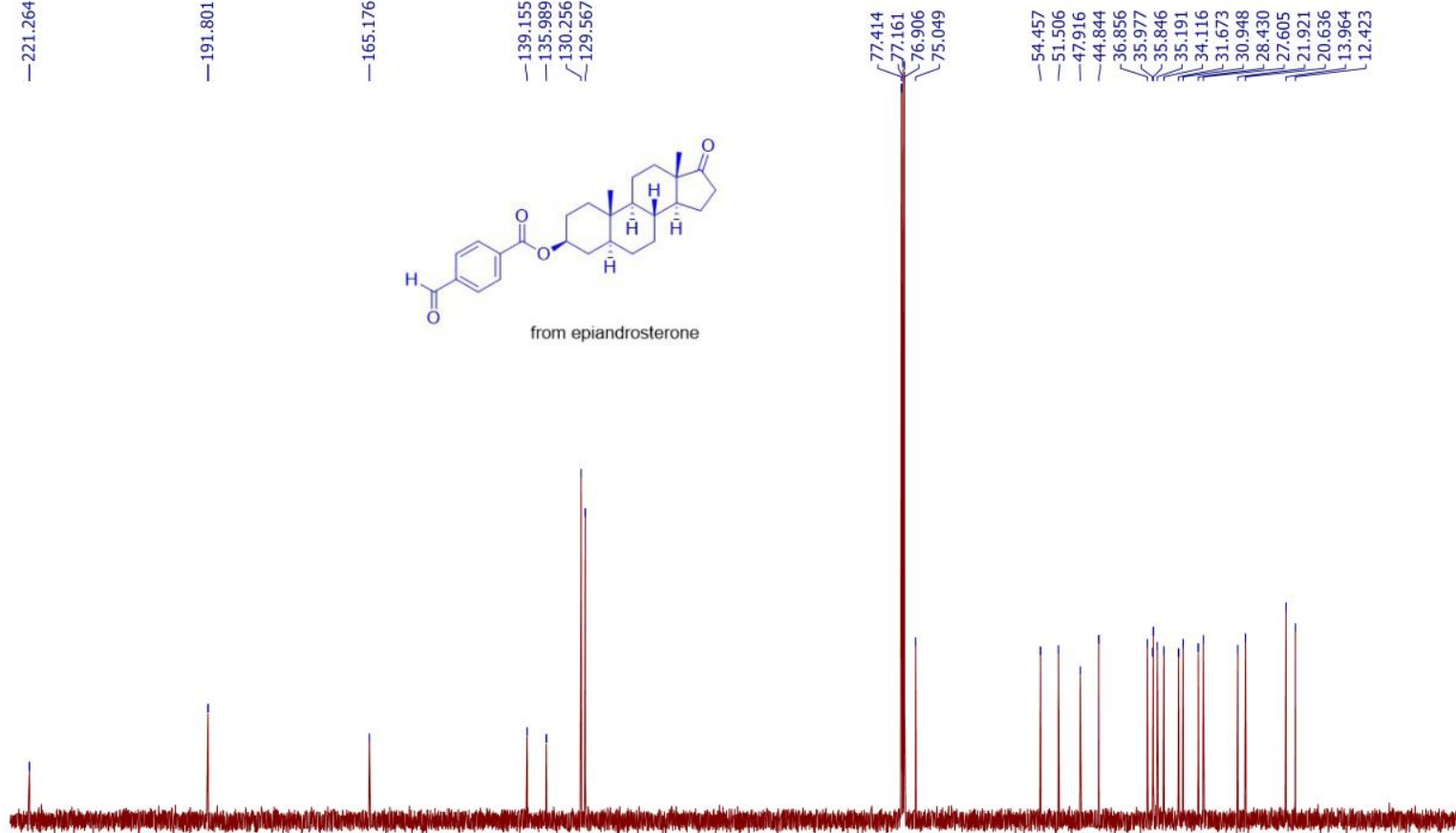

$\begin{array}{lllllllllllllllllllllll}220 & 210 & 200 & 190 & 180 & 170 & 160 & 150 & 140 & 130 & 120 & \begin{array}{c}110 \\ \mathrm{f} 1(\mathrm{ppm})\end{array} & 90 & 80 & 70 & 60 & 50 & 40 & 30 & 20 & 10 & 0 & -10\end{array}$ 


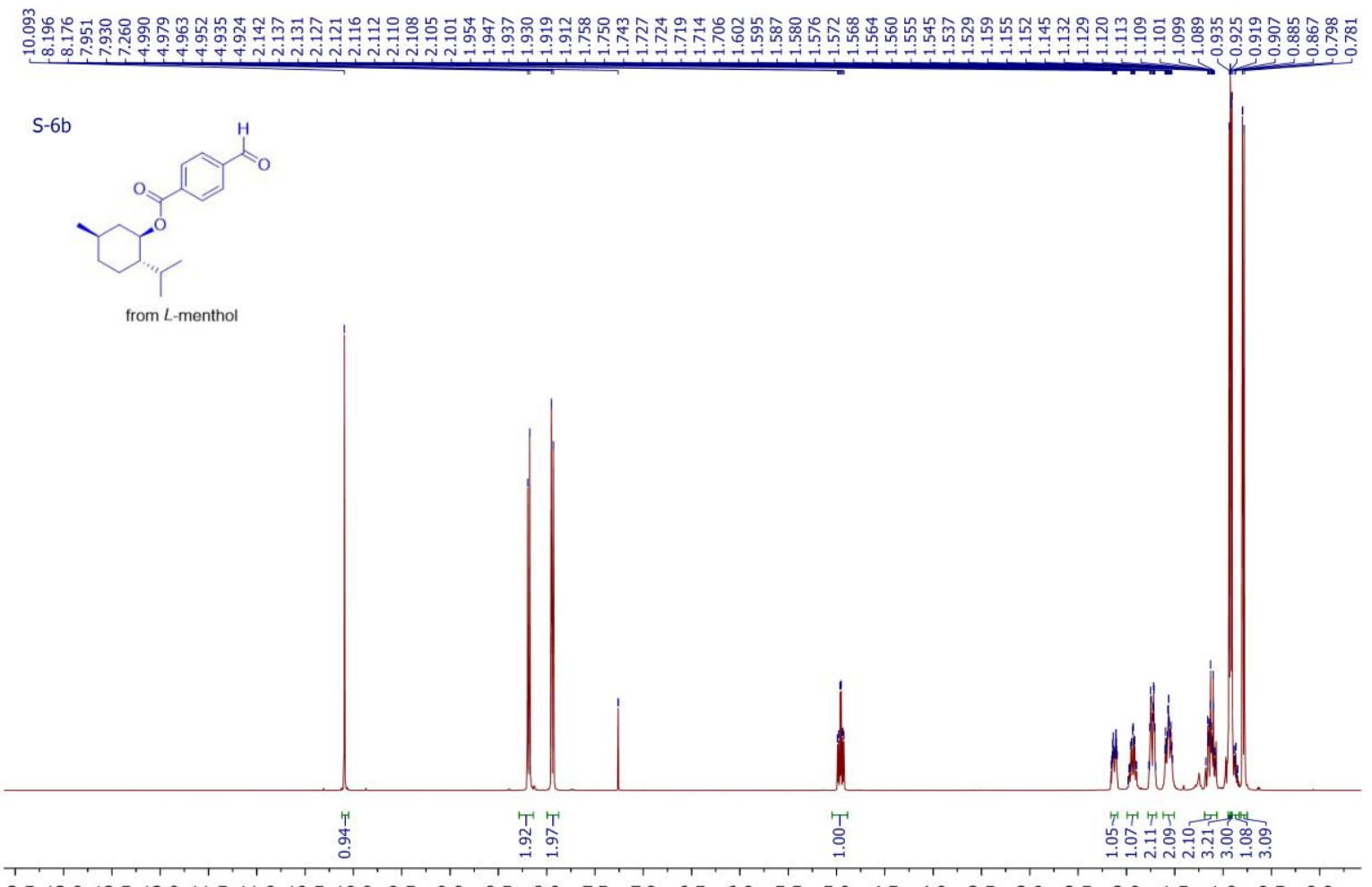

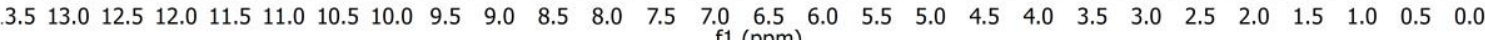

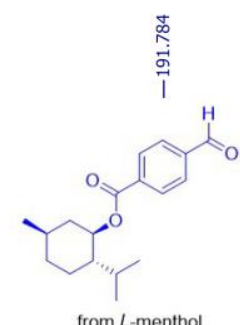

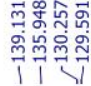

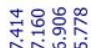

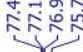

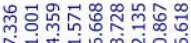

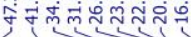

from $L$-menthol

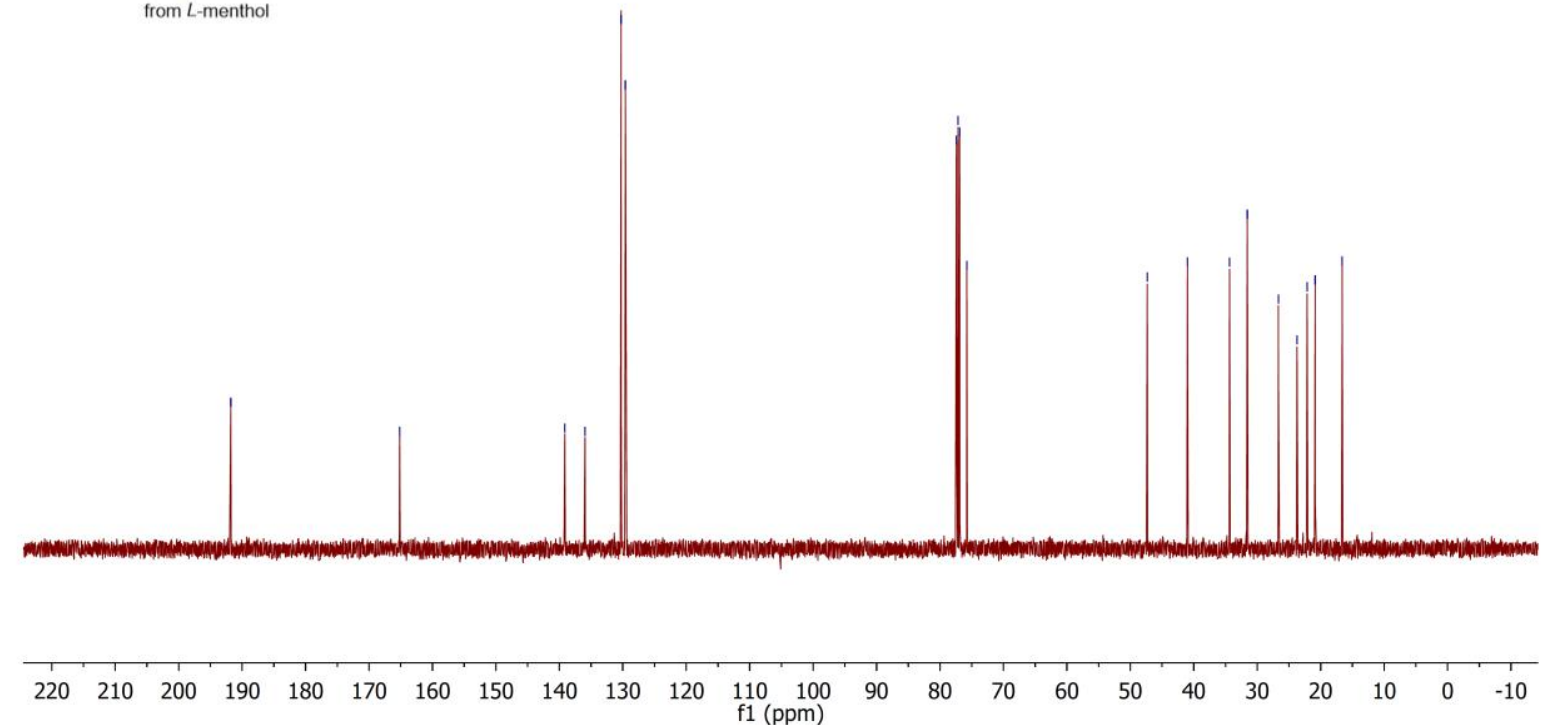



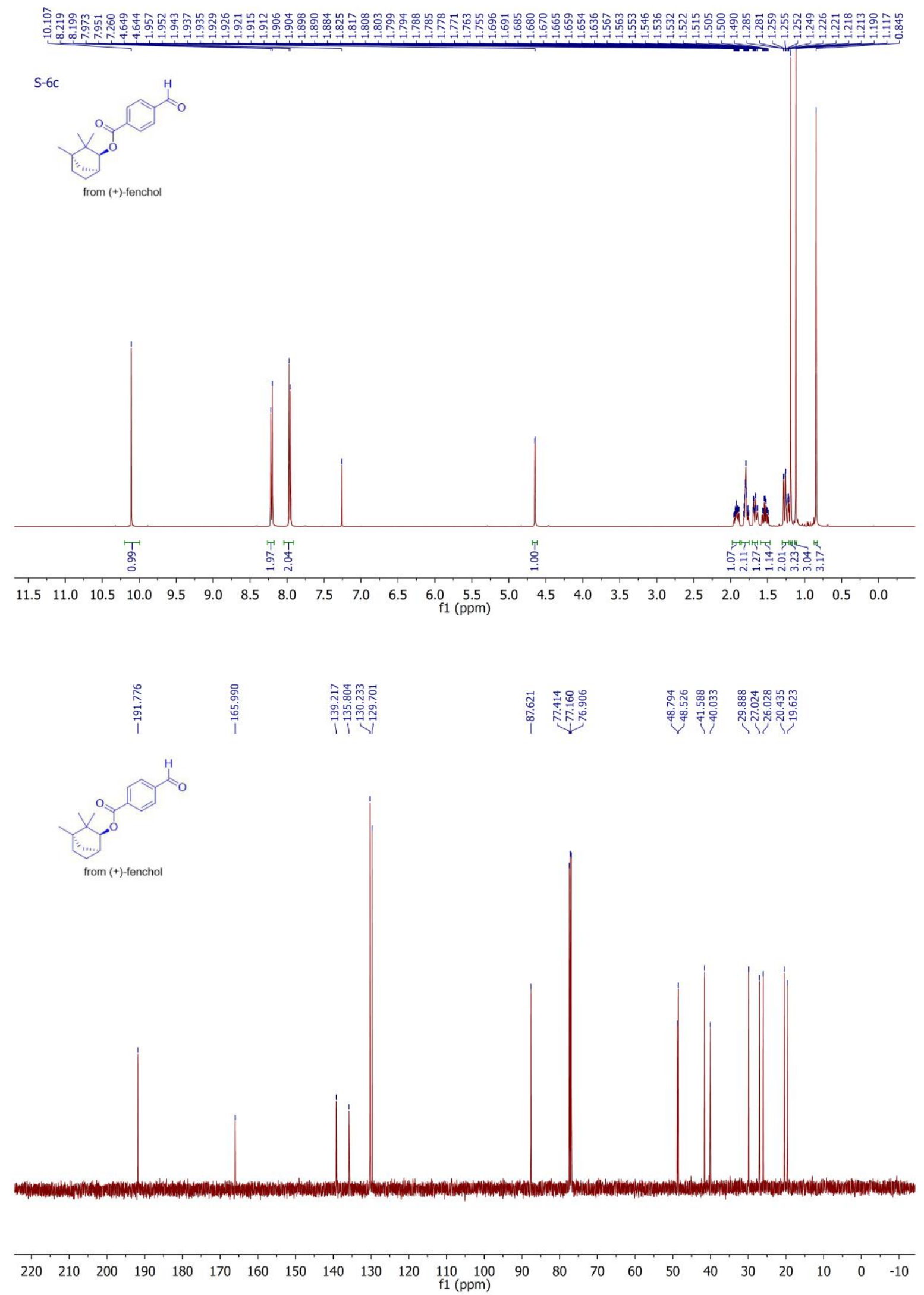


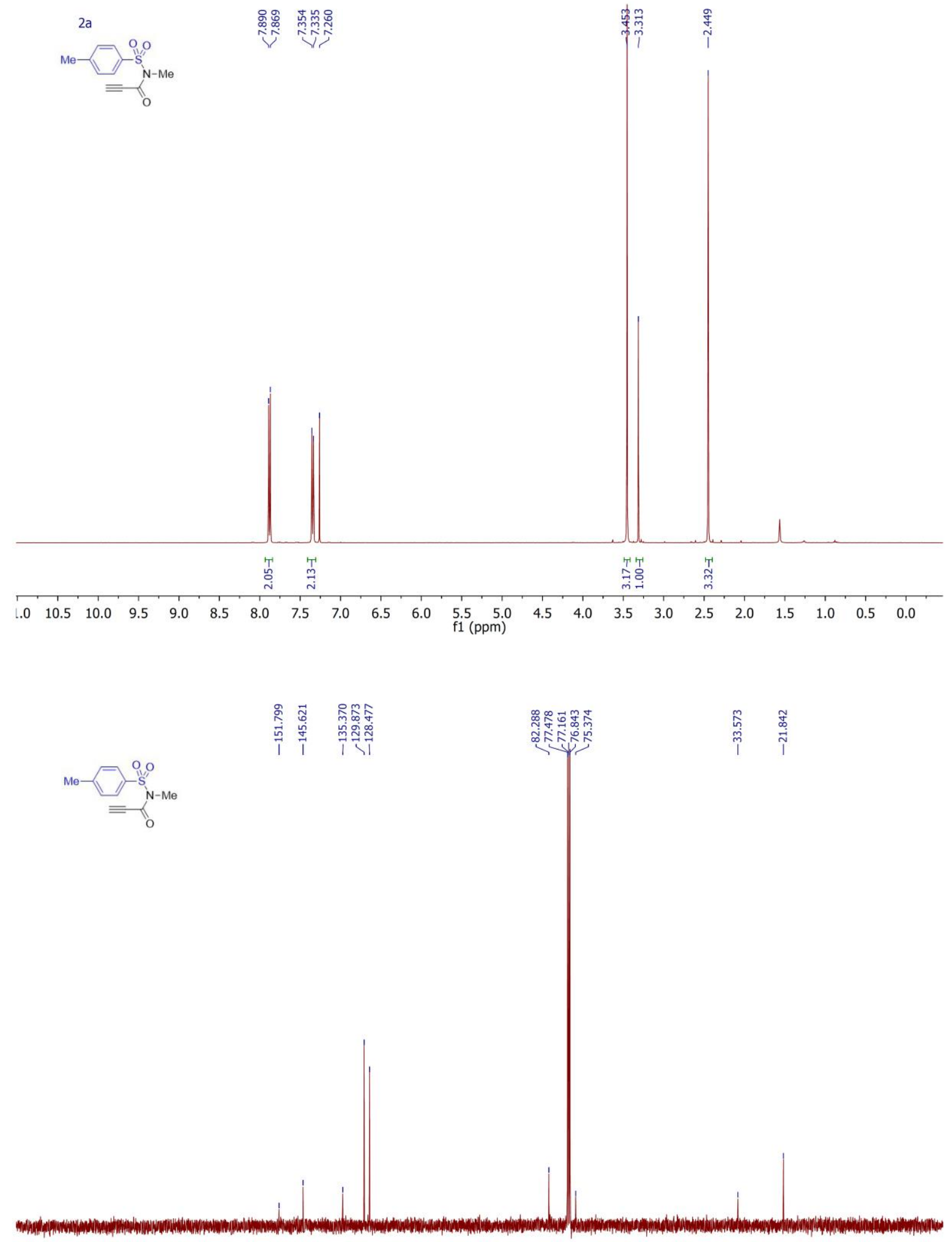

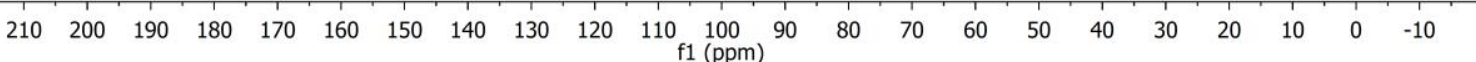



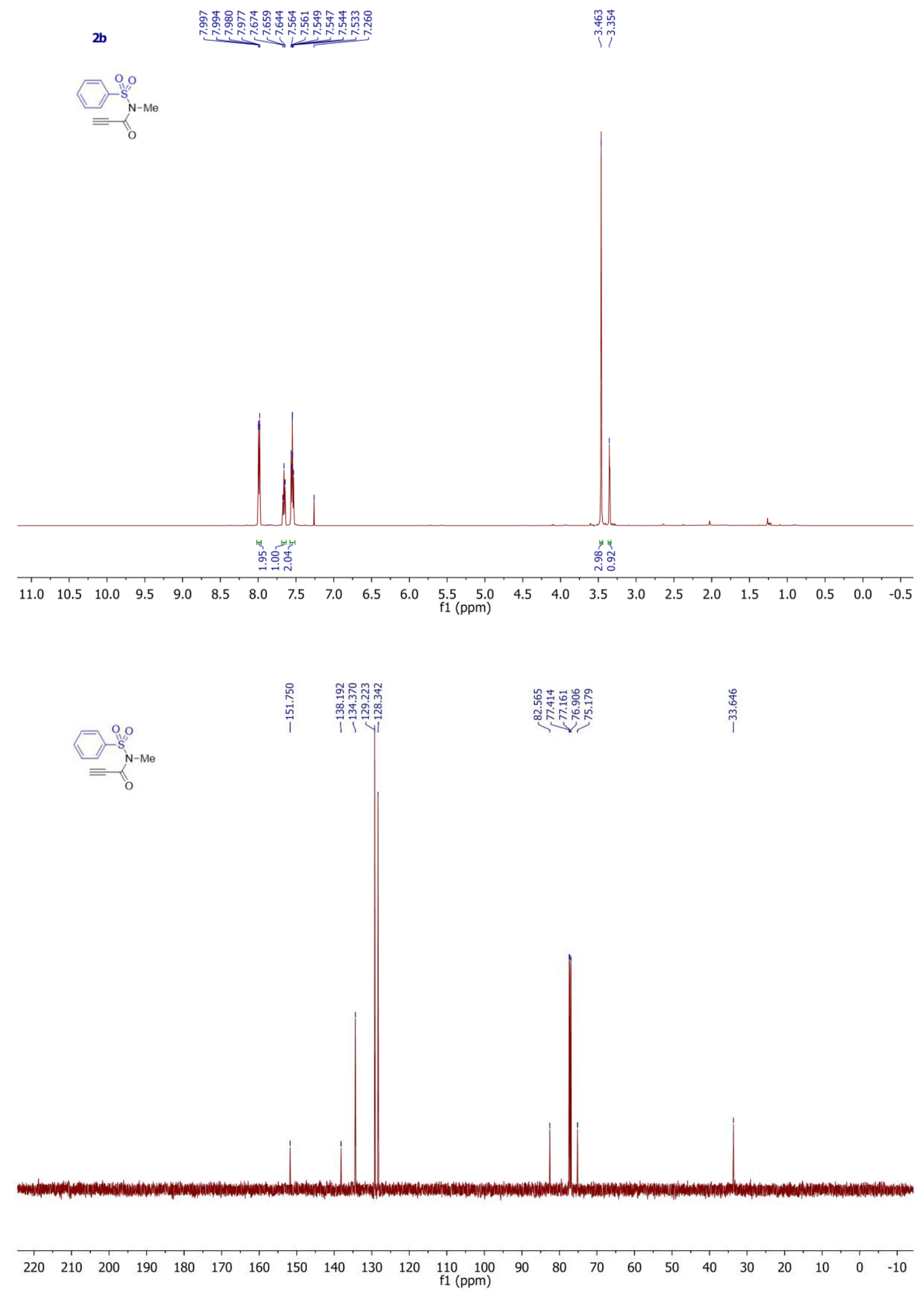

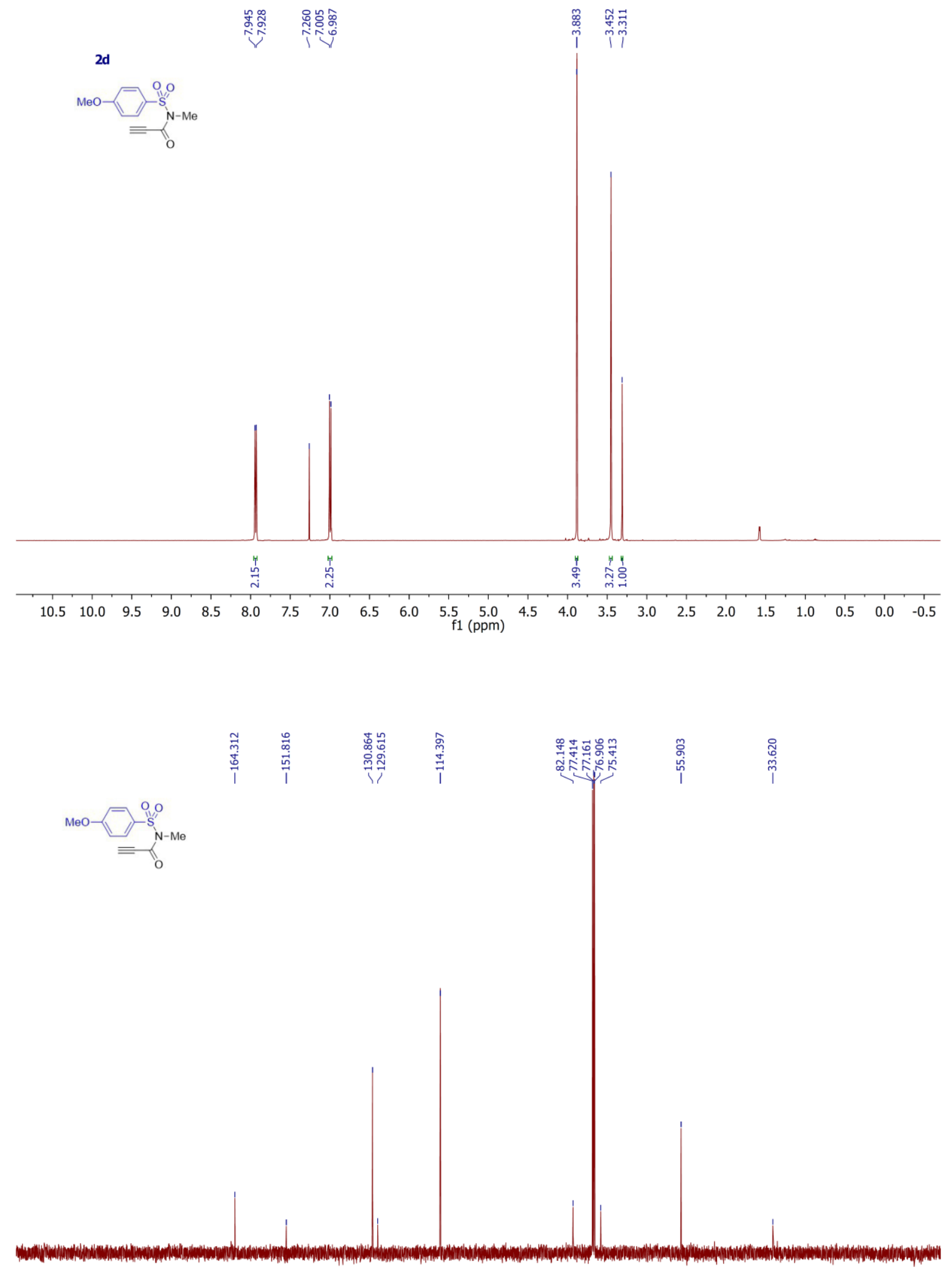

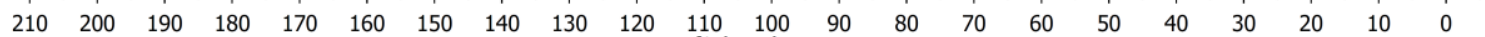




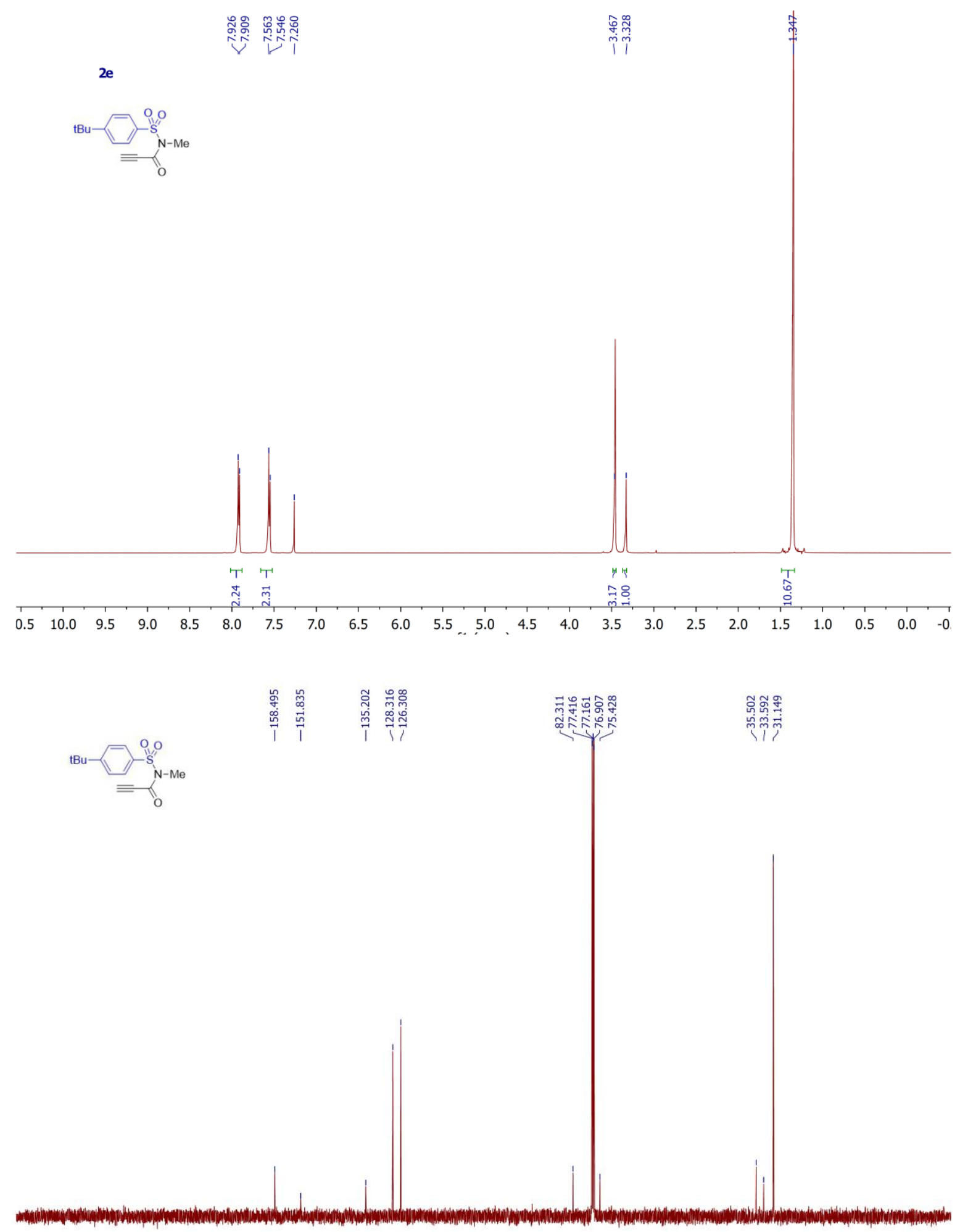

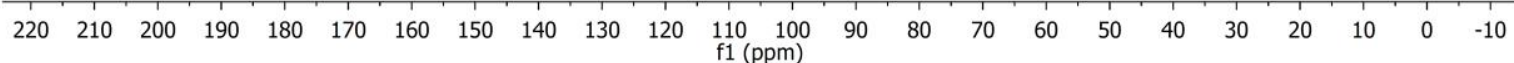



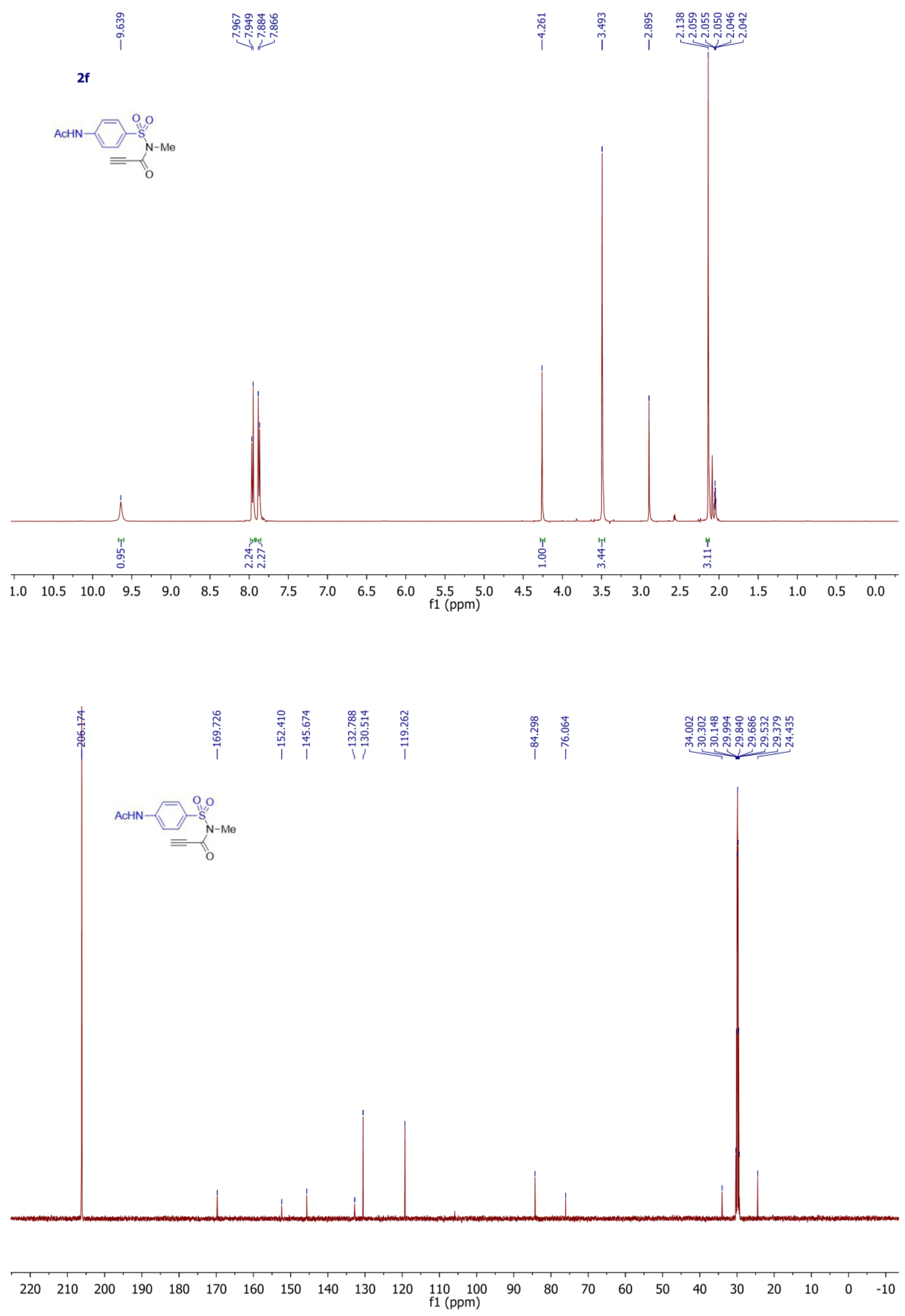

S73 


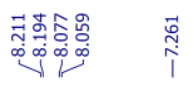

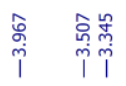

$2 g$
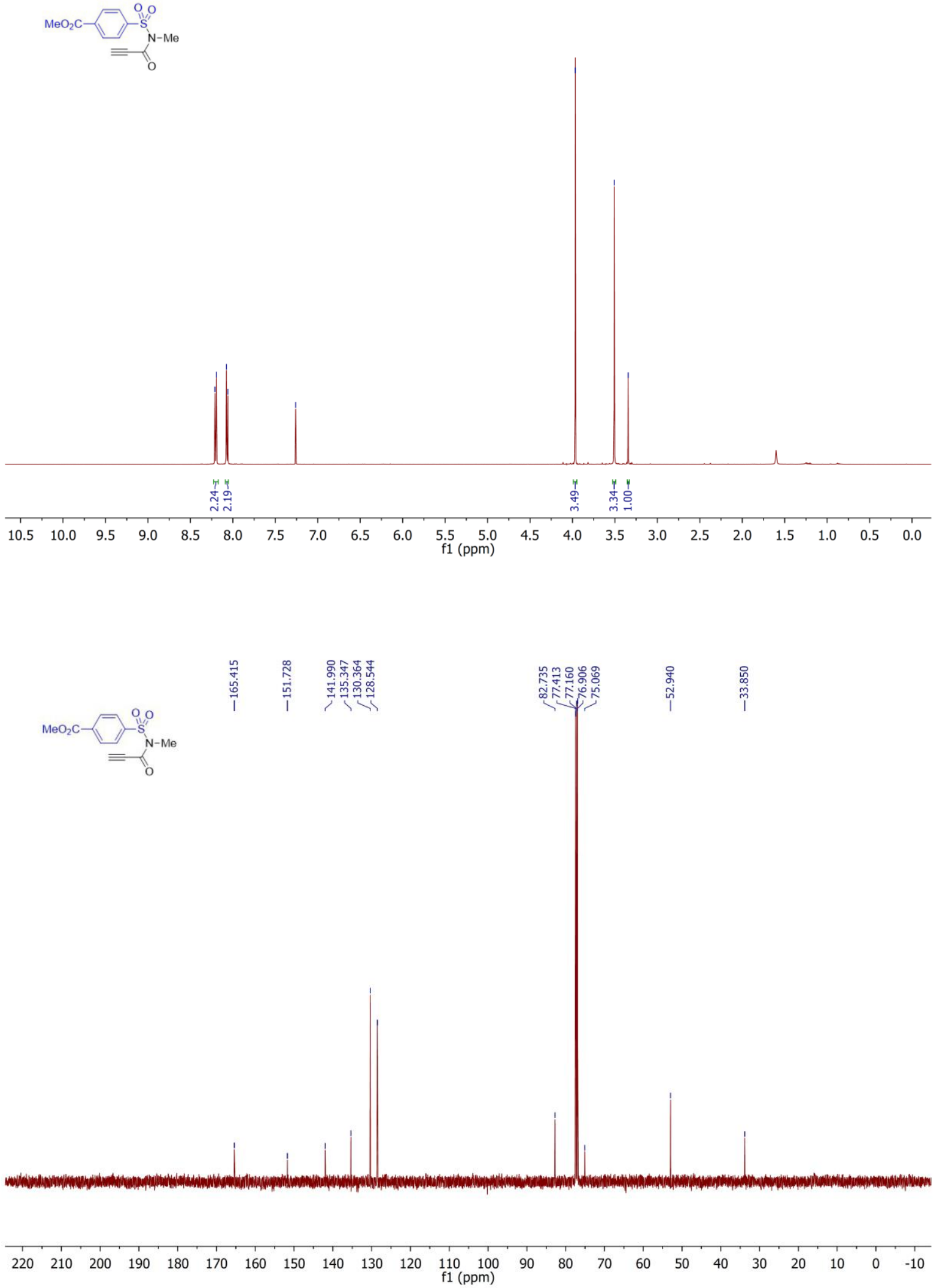

S74 

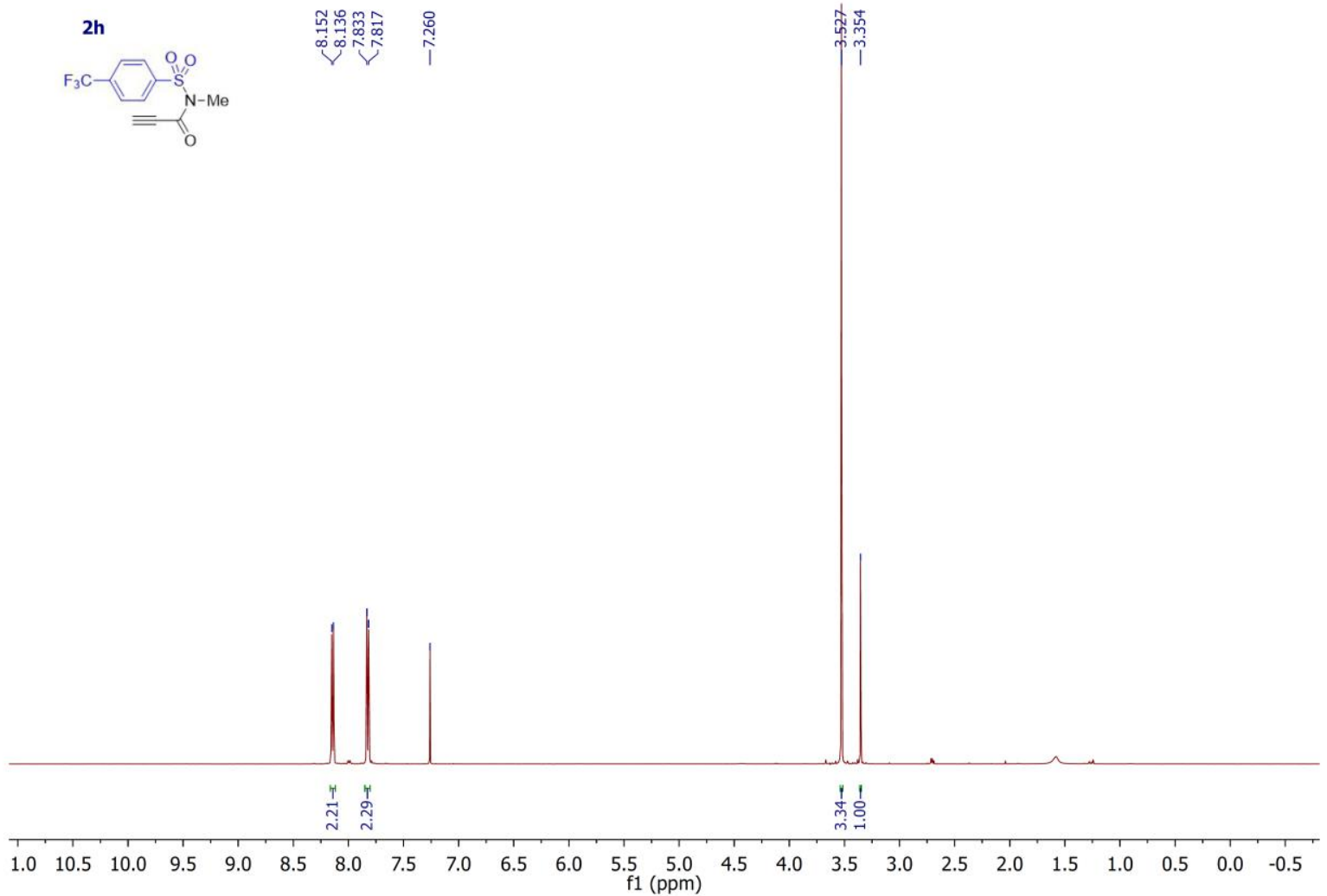

$=\mathrm{O}_{\mathrm{O}}^{\mathrm{N}-\mathrm{Me}}$
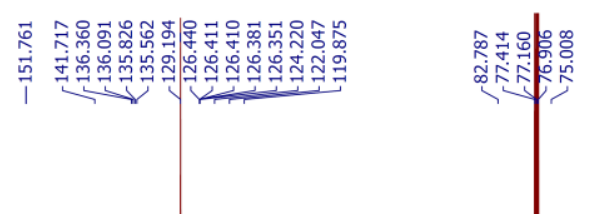

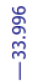

$\begin{array}{lllllllllll}210 & 200 & 190 & 180 & 170 & 160 & 150 & 140 & 130 & 120 & 110 \\ \mathrm{f} 1(\mathrm{ppm}) & 100\end{array}$

$60 \quad 50$

40

$30 \quad 20 \quad 10 \quad 0$ 

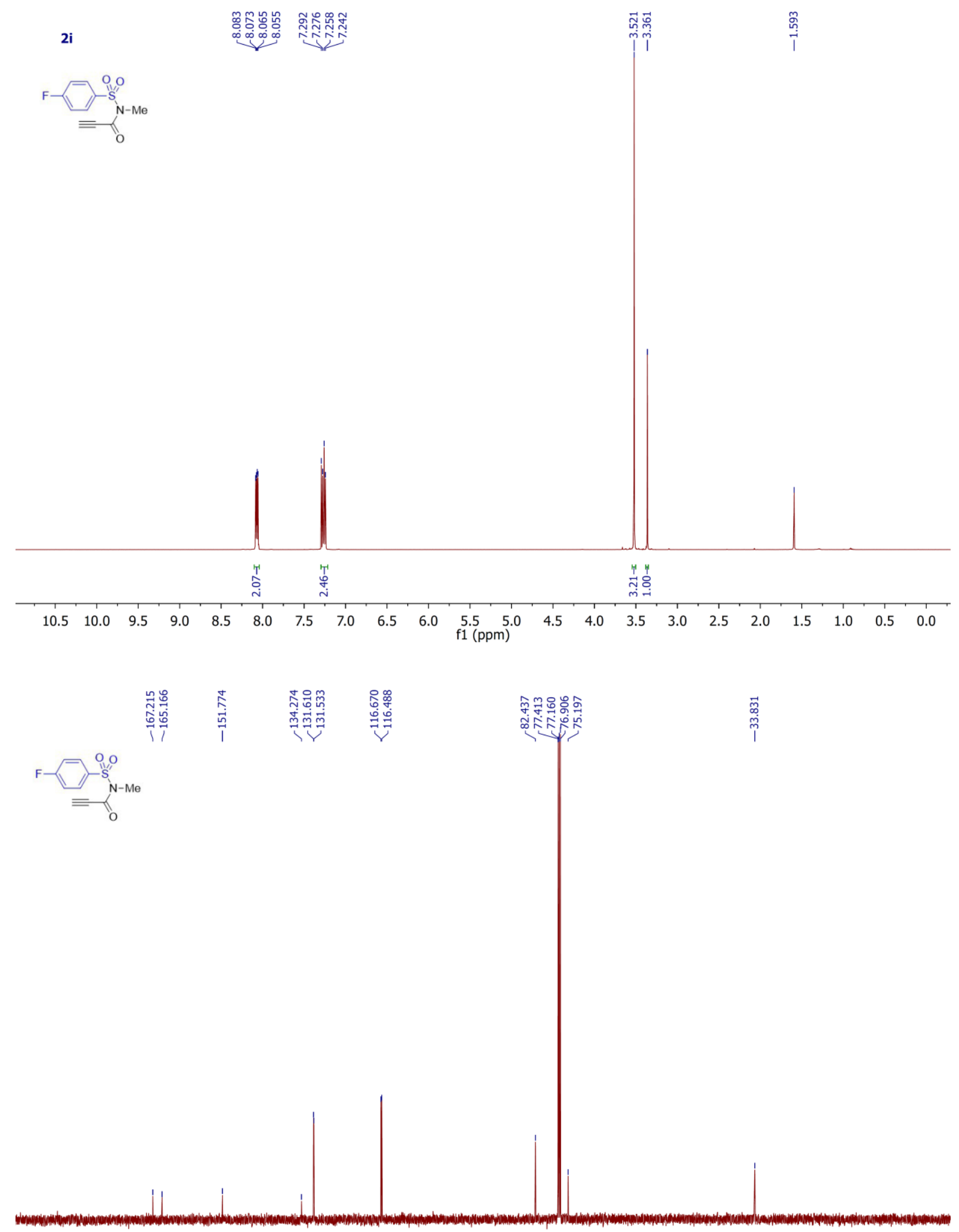

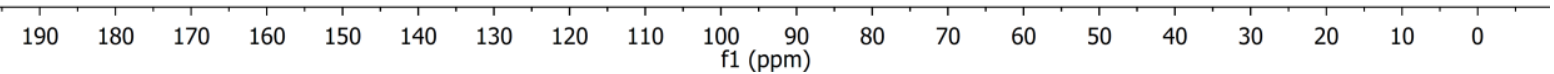


$2 \mathrm{i}$<smiles>C#CC(=O)N(C)S(=O)(=O)c1ccc(F)cc1</smiles>

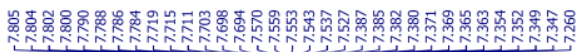

$2 \mathbf{j}$<smiles>C#CC(=O)N(C)S(=O)(=O)c1cccc(F)c1</smiles>
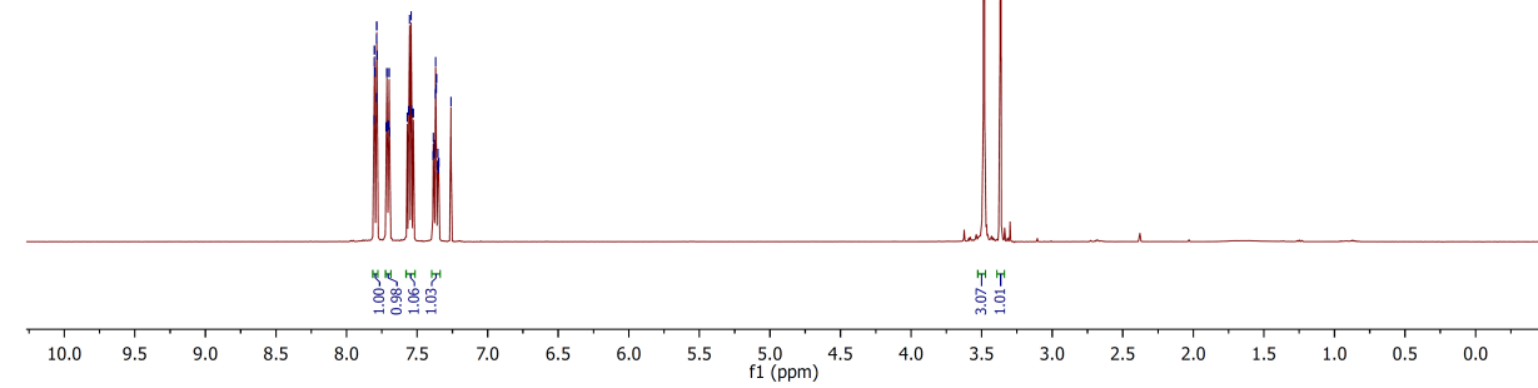

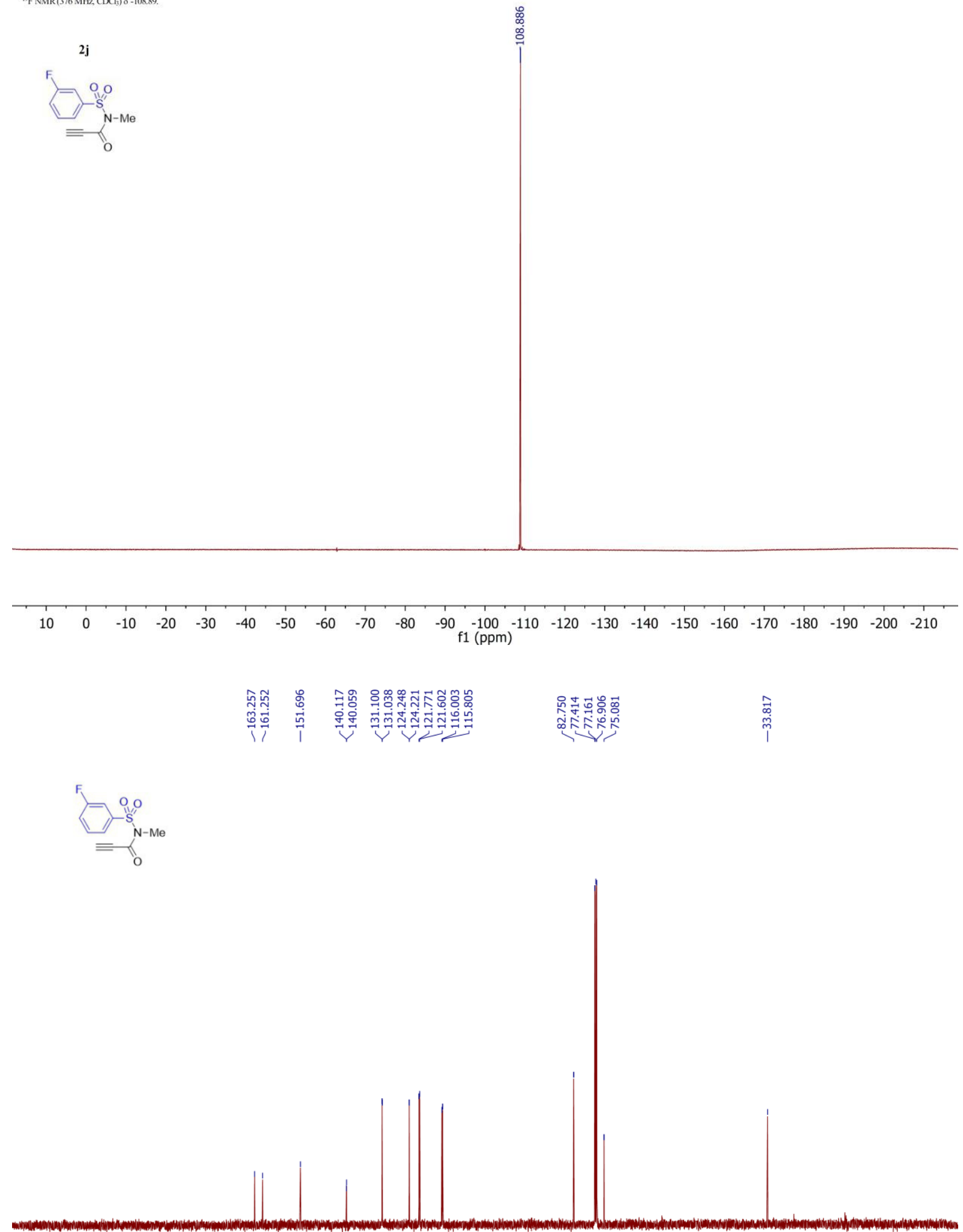

\begin{tabular}{lllllllllllllllllllllllllllllll}
\hline 220 & 210 & 200 & 190 & 180 & 170 & 160 & 150 & 140 & 130 & 120 & $\begin{array}{c}110 \\
\mathrm{f} 1(\mathrm{ppm})\end{array}$ & 100 & 90 & 70 & 60 & 50 & 40 & 30 & 20 & 10 & 0 & -10
\end{tabular} 


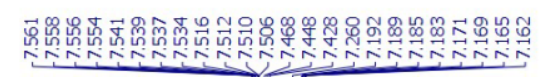

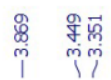

$2 \mathrm{k}$

MeO=

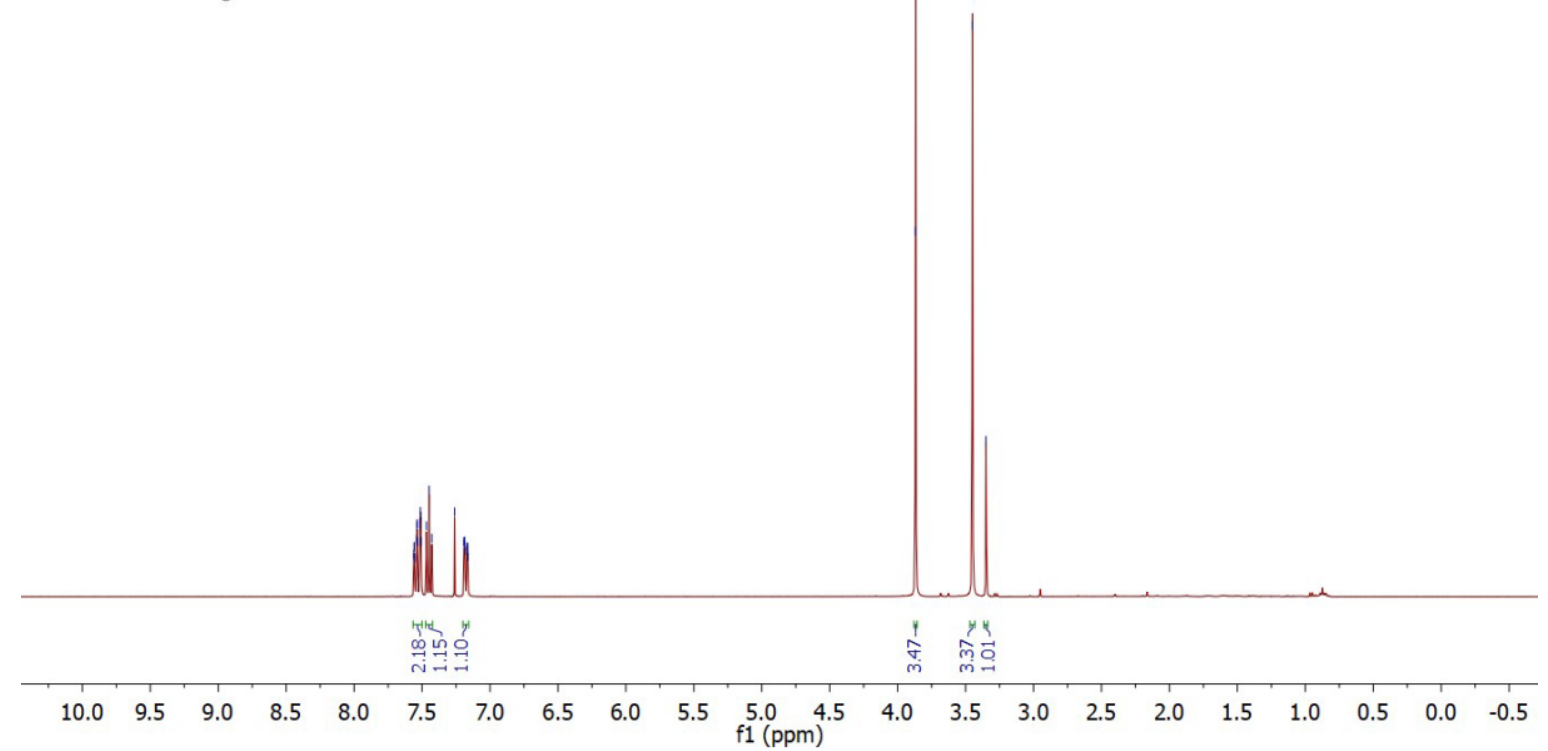

$\stackrel{\text { Ne) }}{\text { MeO }}$

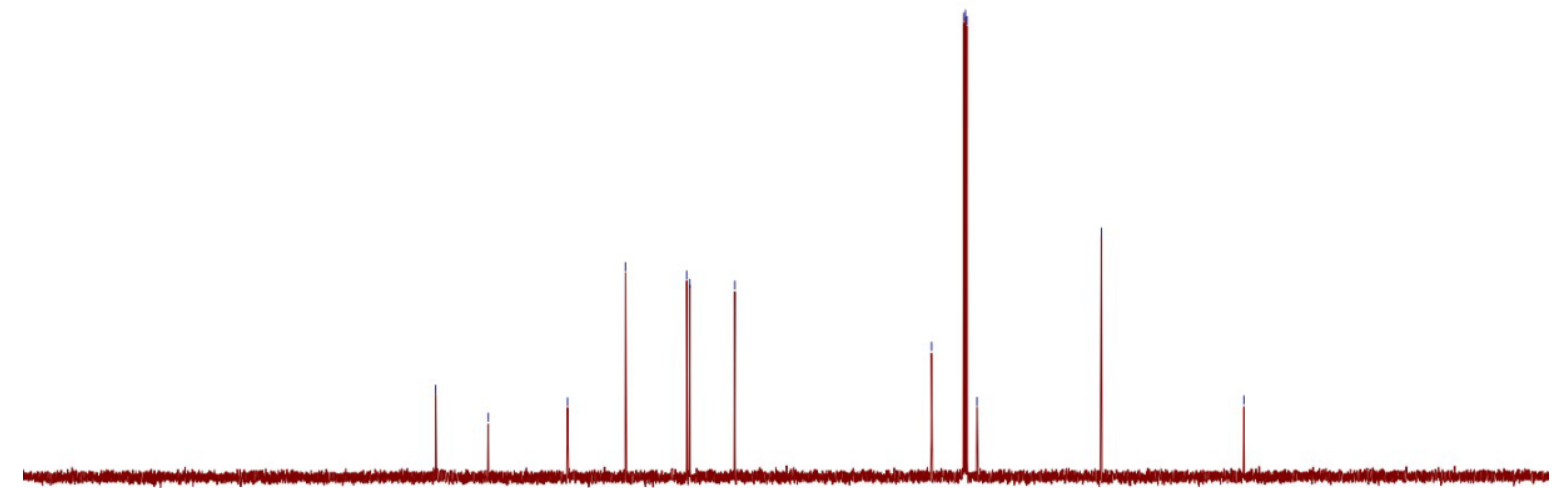

$\begin{array}{lllllllllllll}220 & 210 & 200 & 190 & 180 & 170 & 160 & 150 & 140 & 130 & 120 & 110 & 100\end{array}$ 


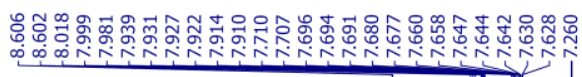

21
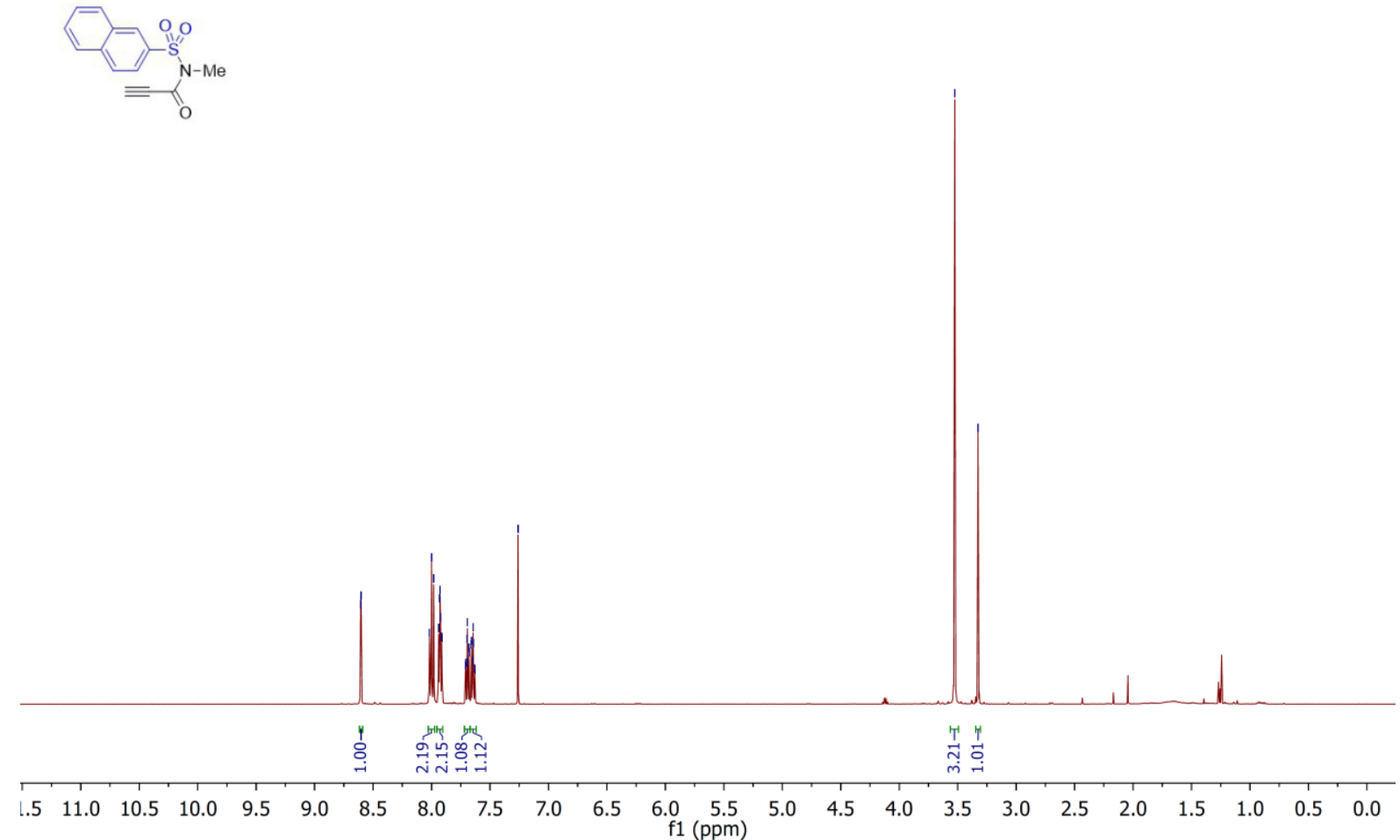

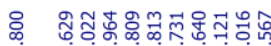

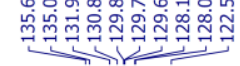

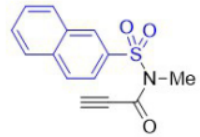
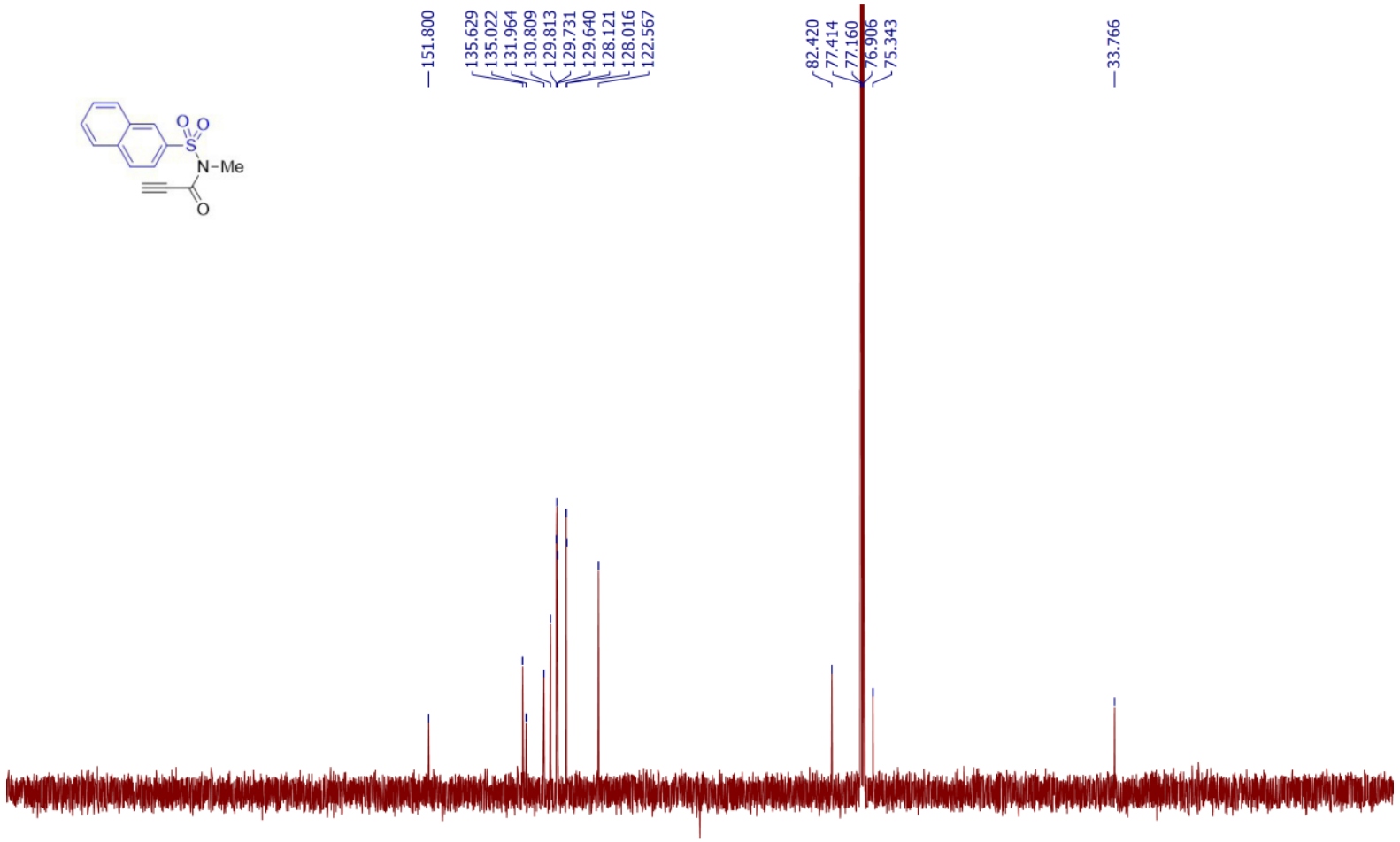

$\begin{array}{lllllllllllllllllllllllll}220 & 210 & 200 & 190 & 180 & 170 & 160 & 150 & 140 & 130 & 120 & \begin{array}{l}110 \\ \mathrm{f} 1(\mathrm{ppm})\end{array} & 90 & 80 & 70 & 60 & 50 & 40 & 30 & 20 & 10 & 0 & -10\end{array}$ 

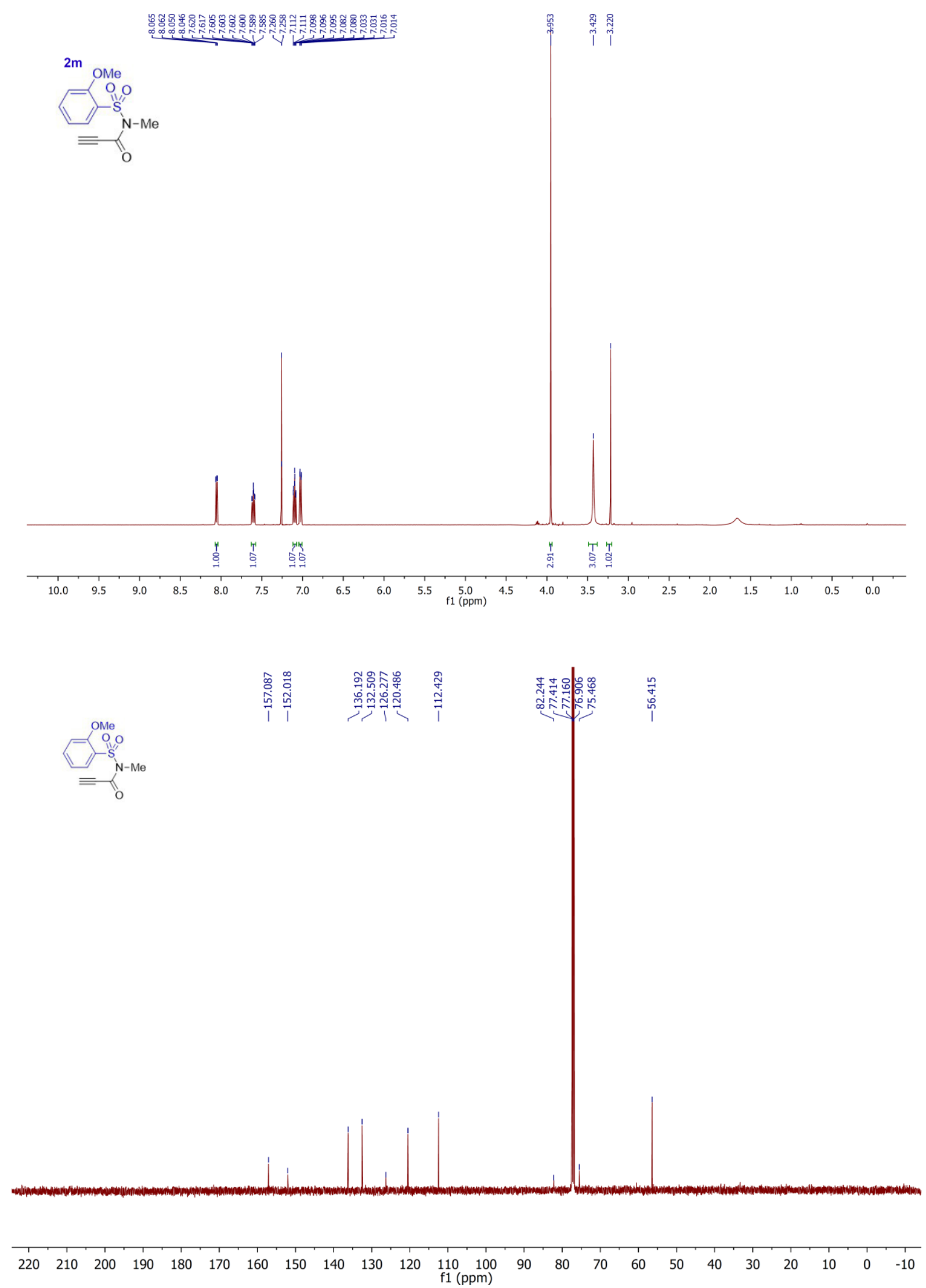

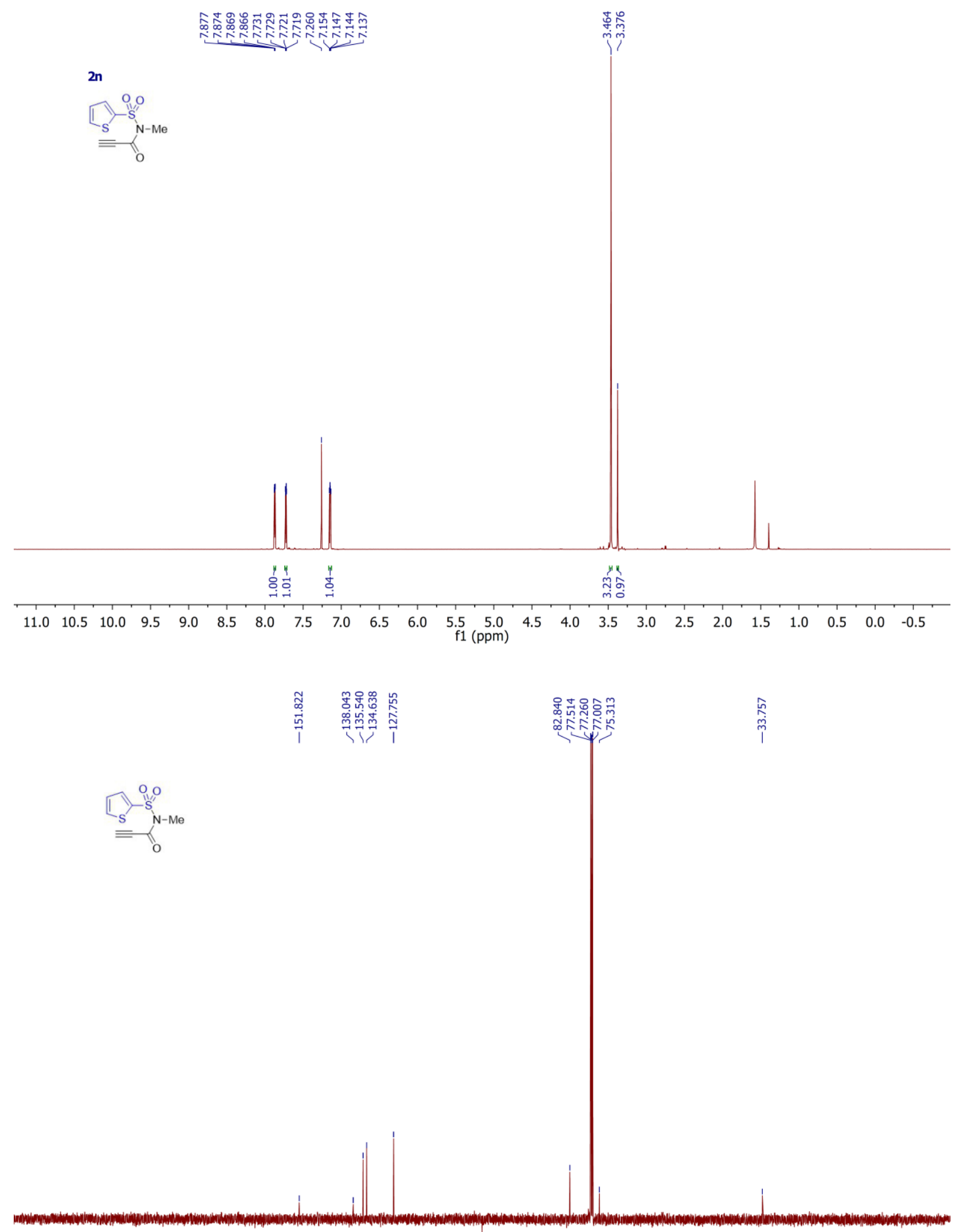

$\begin{array}{lllllllllllllllllllllllll}220 & 210 & 200 & 190 & 180 & 170 & 160 & 150 & 140 & 130 & 120 & 110 & 100 & 90 & 80 & 70 & 60 & 50 & 40 & 30 & 20 & 10 & 0 & -10\end{array}$ 

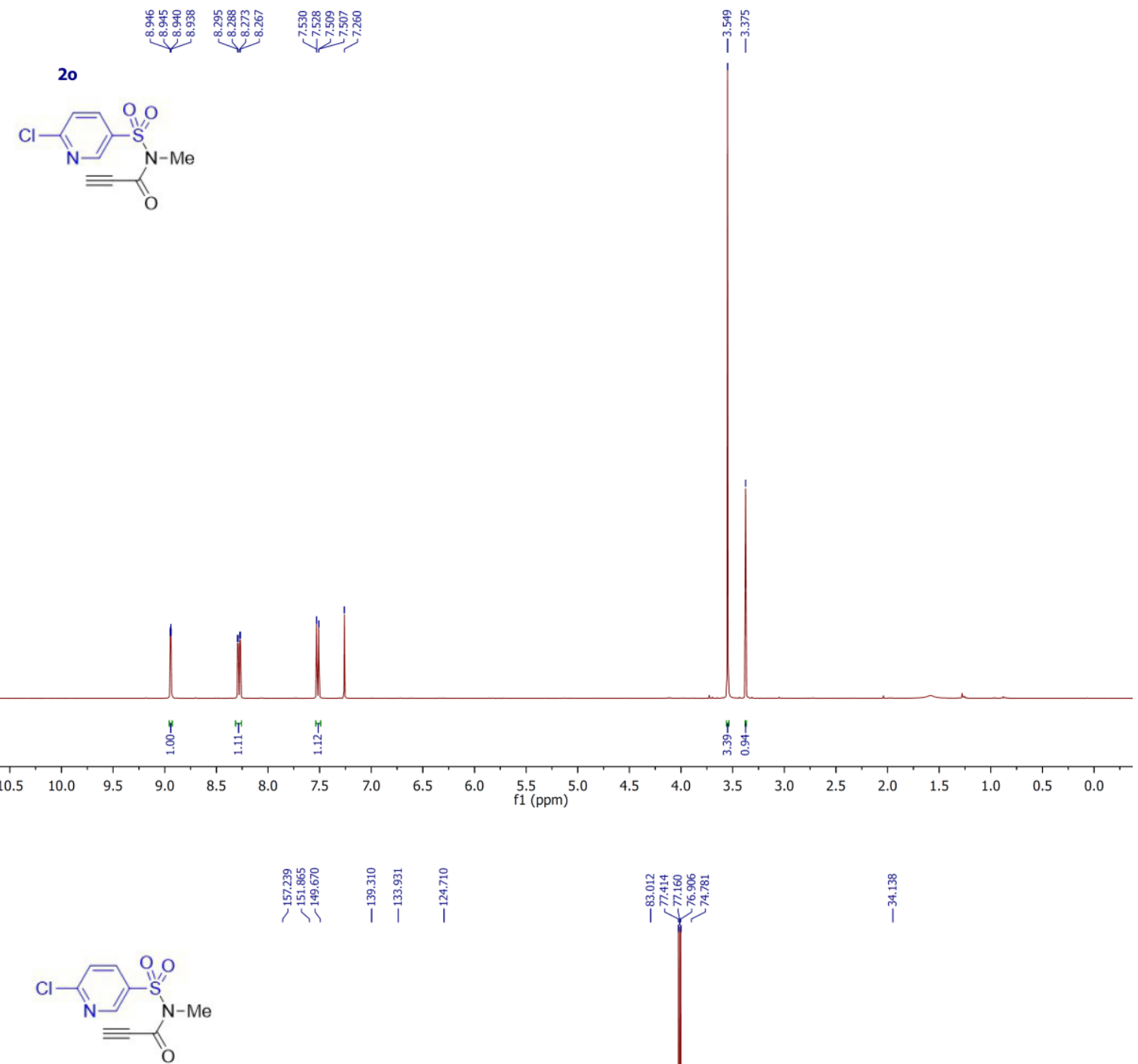

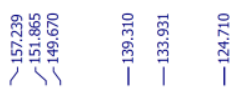

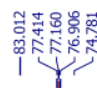

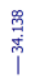

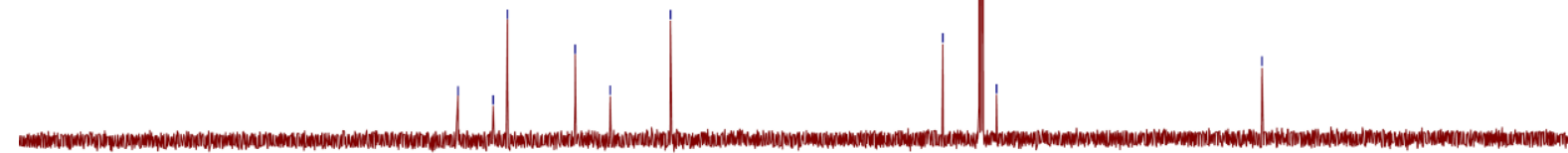

$\begin{array}{llllllllllll}220 & 210 & 200 & 190 & 180 & 170 & 160 & 150 & 140 & 130 & 120 & 110 \\ \mathrm{f} 1(\mathrm{ppm})\end{array}$ 


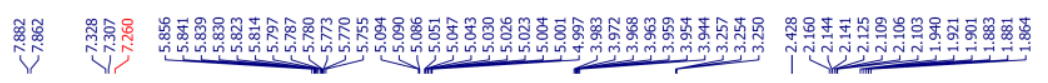<smiles>C#CC(=O)N(CCCC=C)S(=O)(=O)c1ccc(C)cc1</smiles>

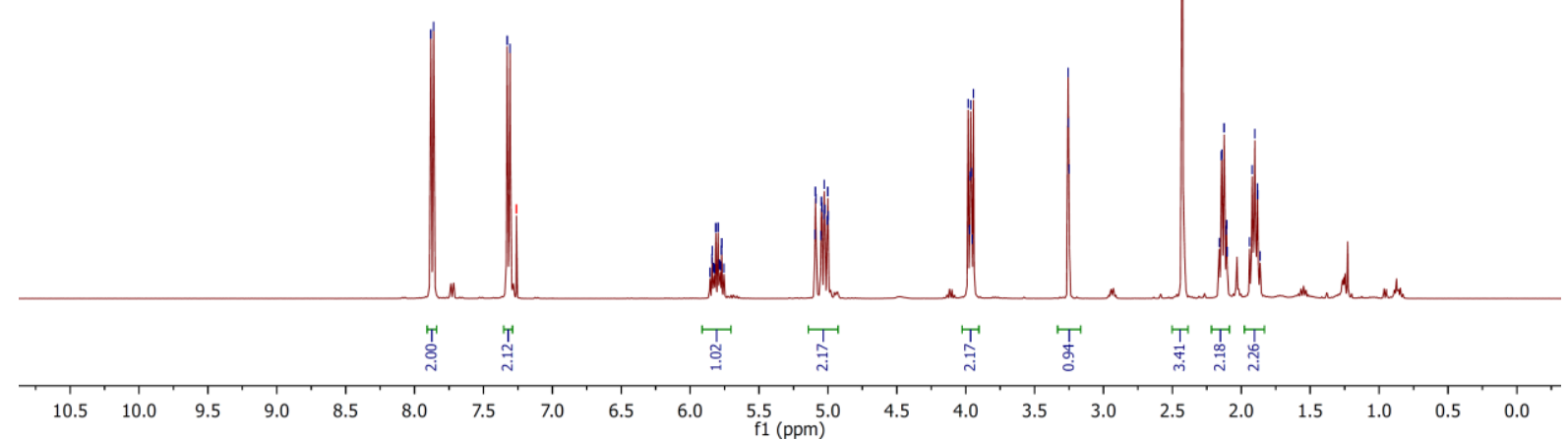<smiles>C#CC(=O)N(CCCC=C)S(=O)(=O)c1ccc(C)cc1</smiles> 


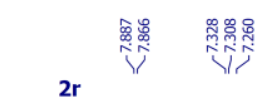

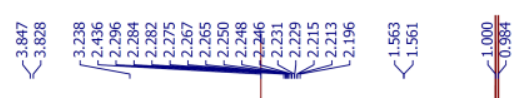<smiles>C#CC(=O)N(CC(C)C)S(=O)(=O)c1ccc(C)cc1</smiles>

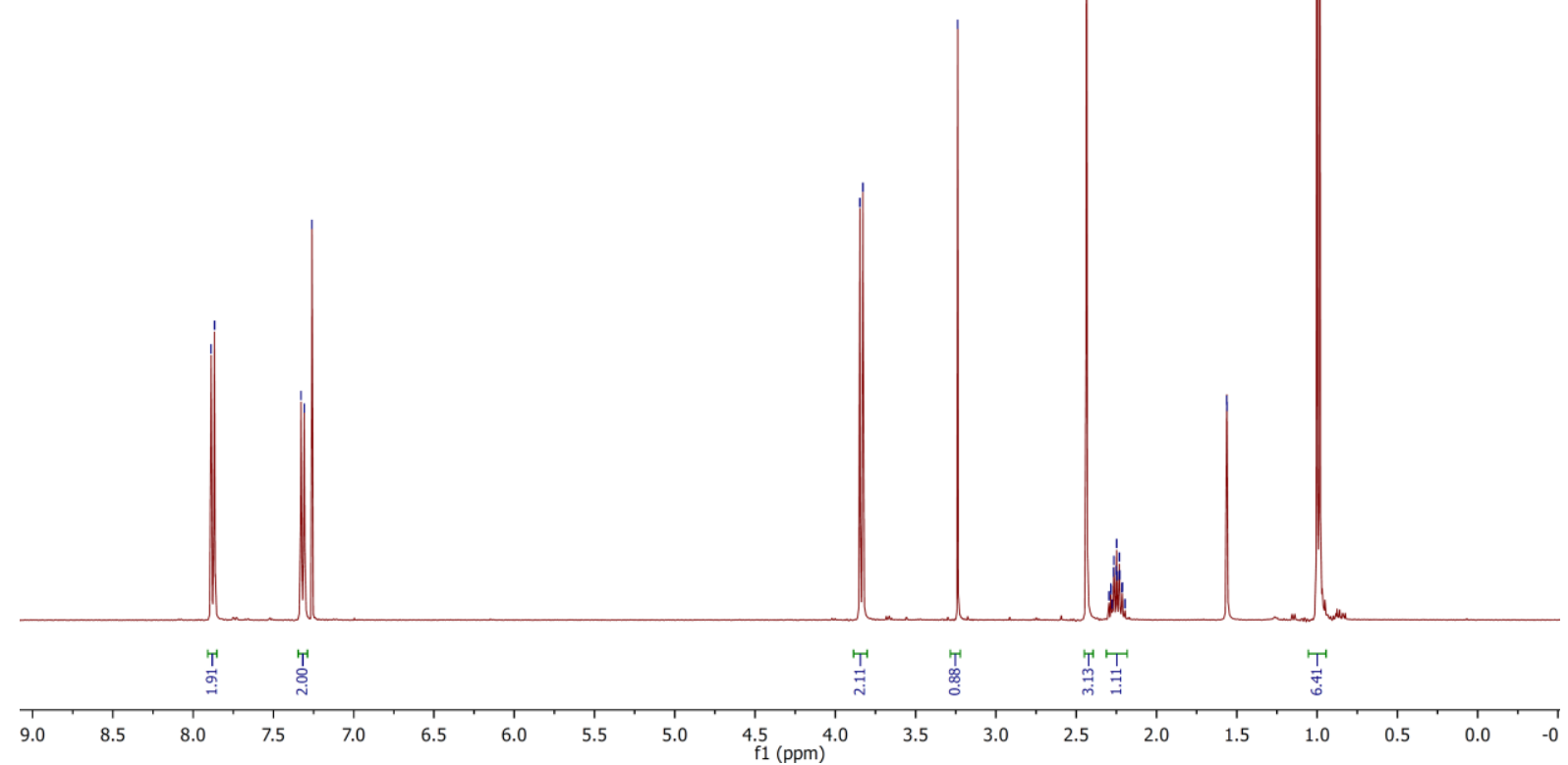<smiles>C#CC(=O)N(CC(C)C)S(=O)(=O)c1ccc(C)cc1</smiles> 


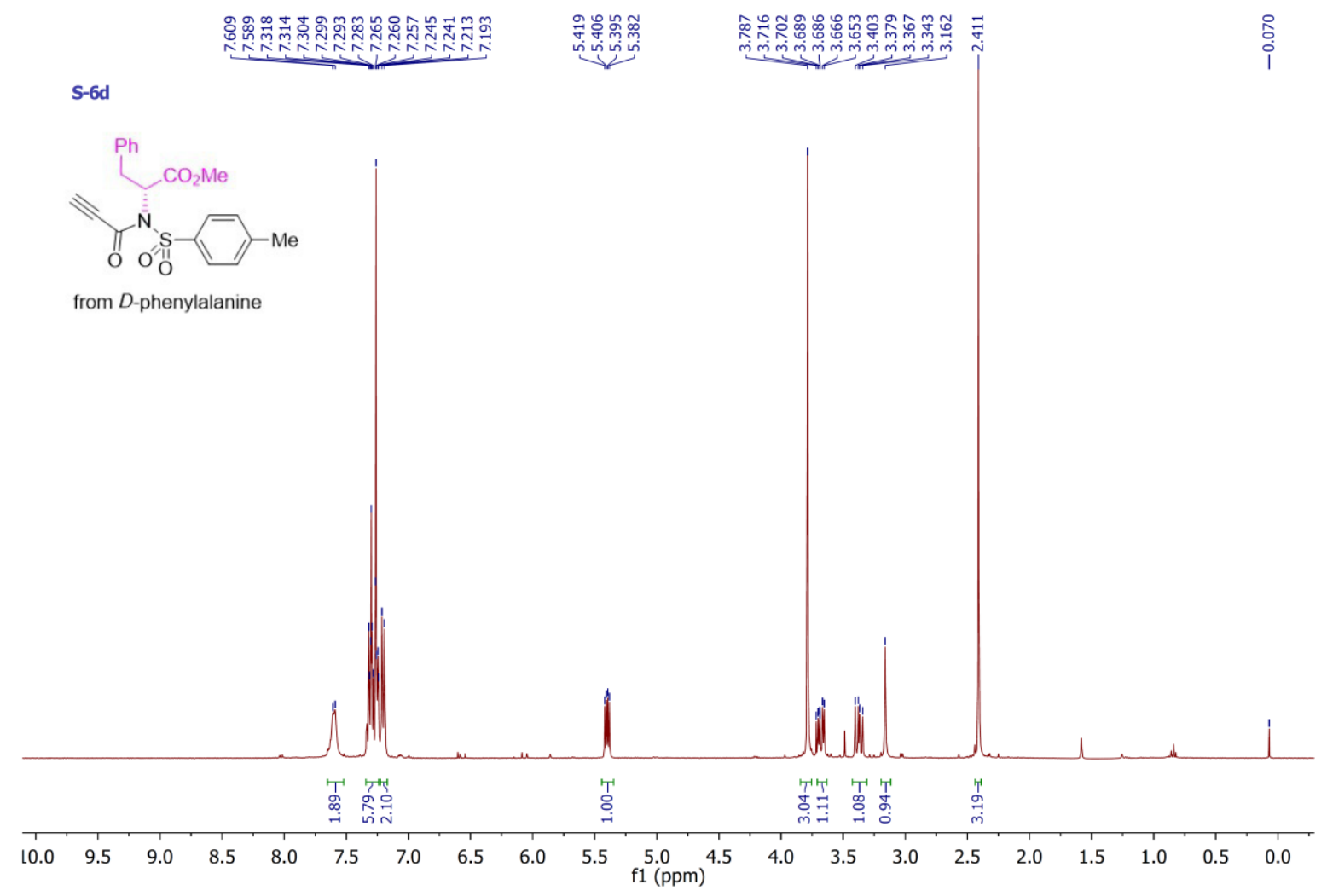

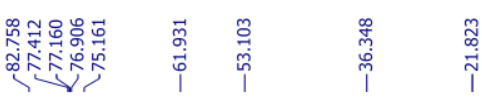

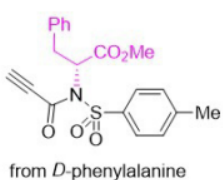

from $D$-phenylalanine
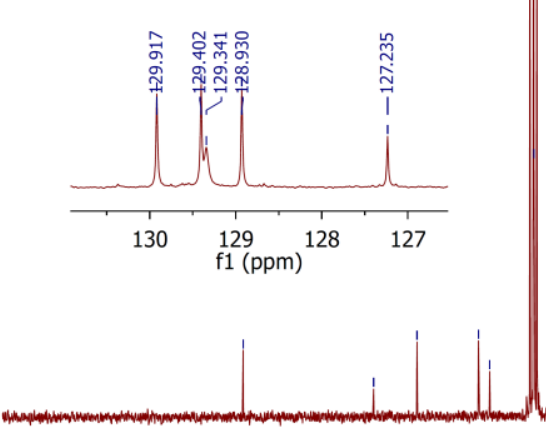

200190

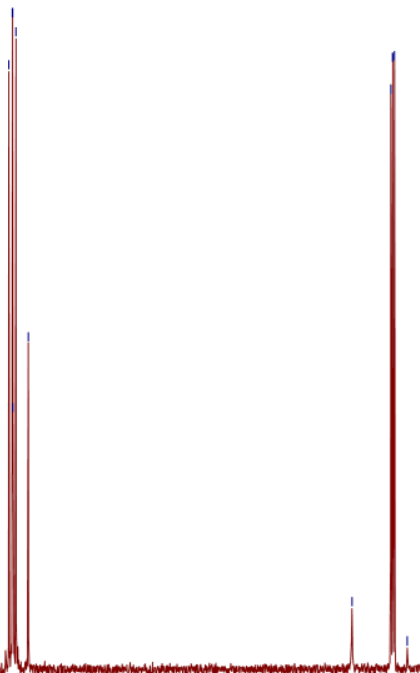

点

ज़

$75 \quad 70 \underset{\mathrm{f} 1(\mathrm{ppm})}{65} 60$

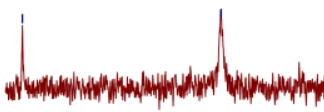




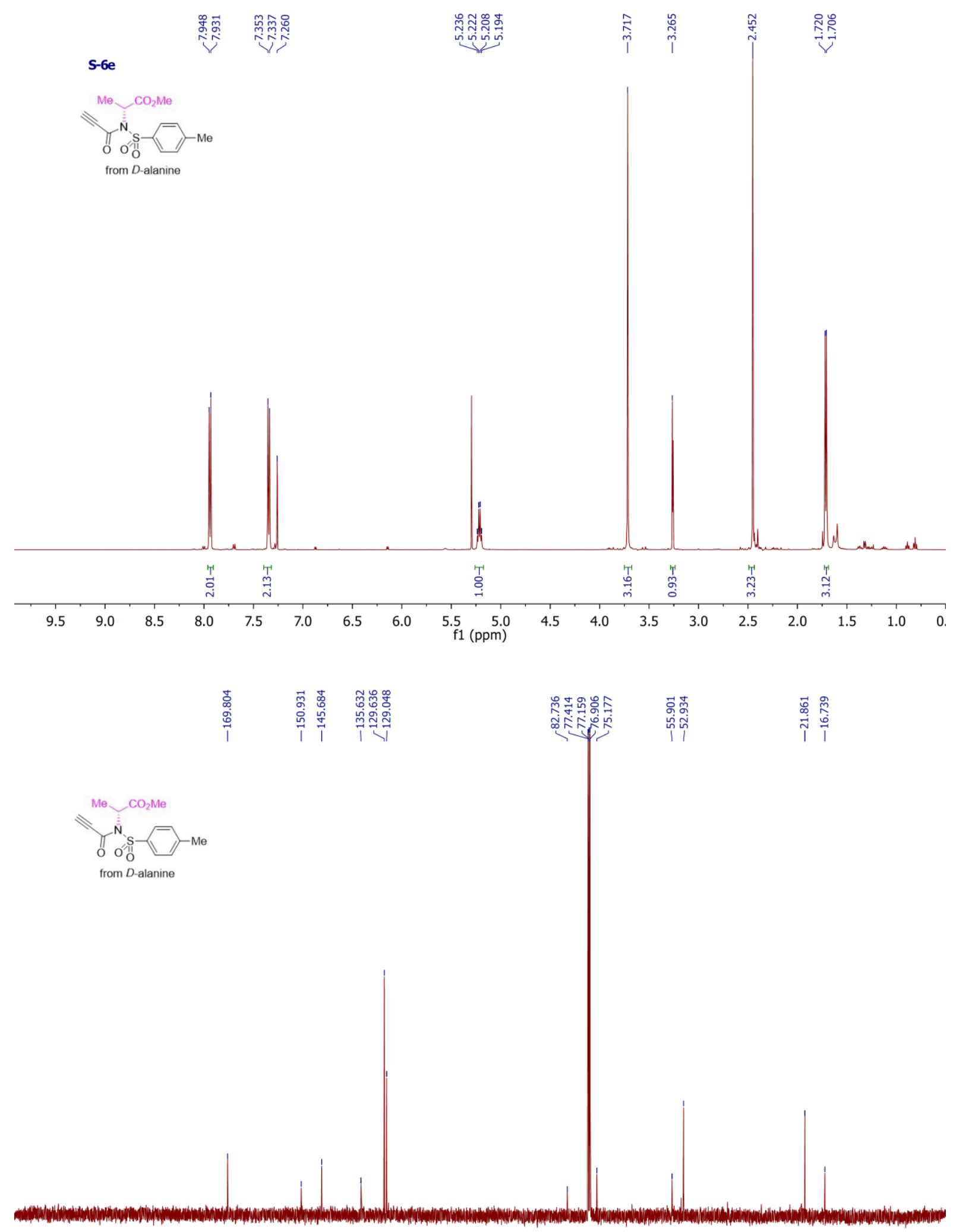

\begin{tabular}{llllllllllllllllllllllllllll}
\hline & 220 & 210 & 200 & 190 & 180 & 170 & 160 & 150 & 140 & 130 & 120 & 110 & 100 & 90 & 80 & 70 & 60 & 50 & 40 & 30 & 20 & 10 & 0 & -10
\end{tabular} 

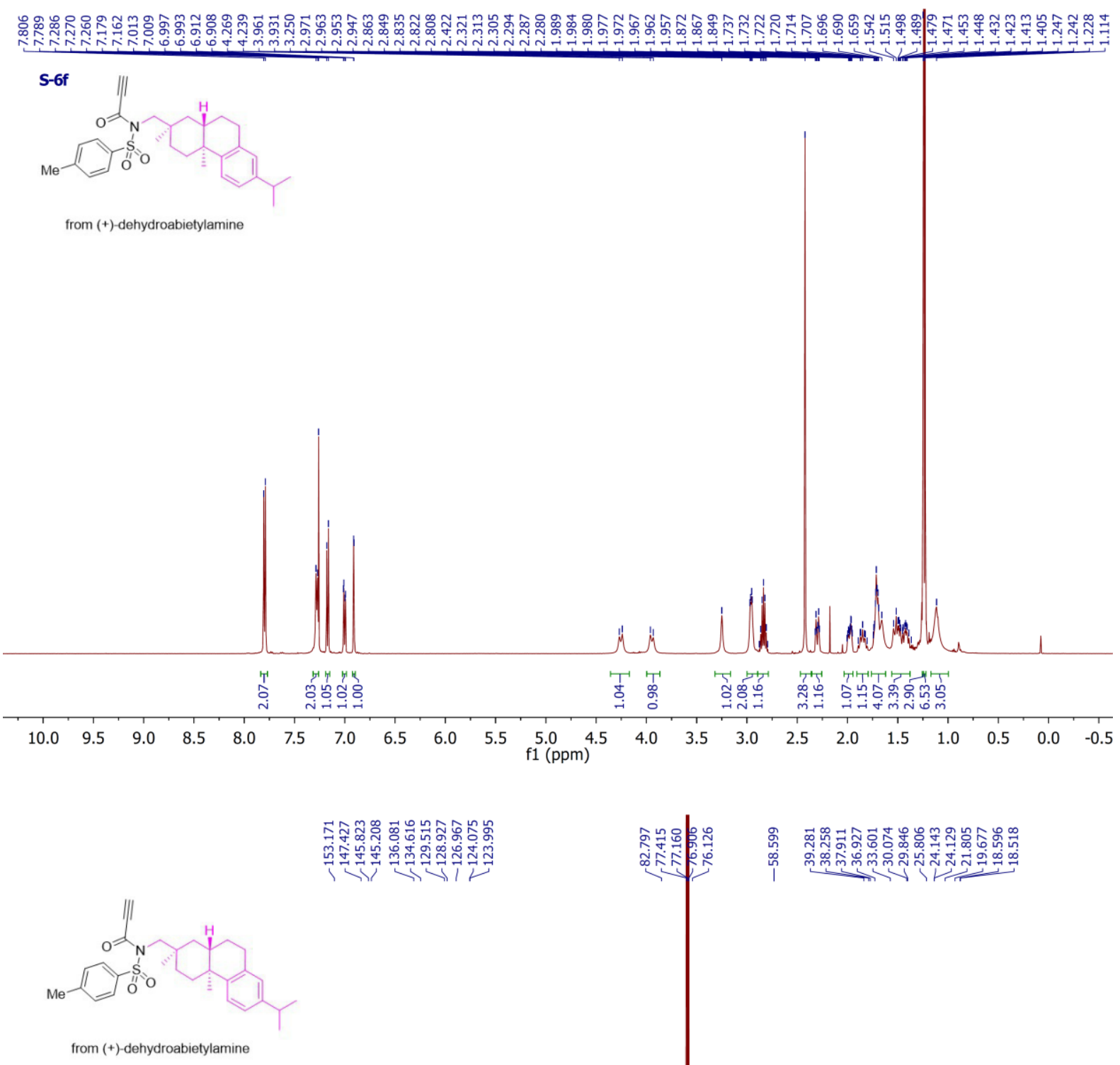

\begin{tabular}{llllllllllllllllllllllllllll}
\hline 220 & 210 & 200 & 190 & 180 & 170 & 160 & 150 & 140 & 130 & 120 & $\begin{array}{l}110 \\
\mathrm{f} 1(\mathrm{ppm})\end{array}$ & 100 & 90 & 80 & 70 & 60 & 50 & 40 & 30 & 20 & 10 & 0 & -10
\end{tabular} 

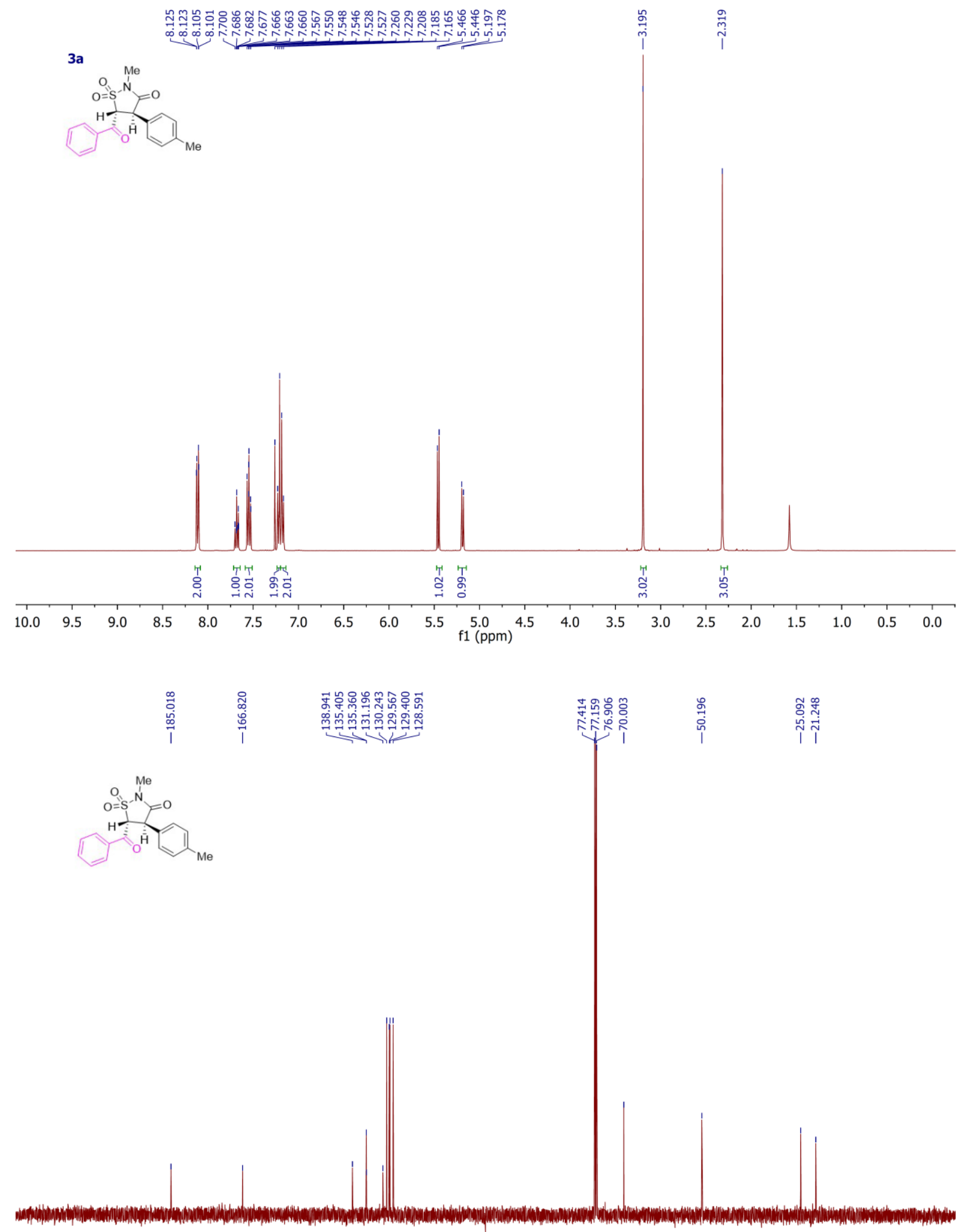

$\begin{array}{rlllllllllllllllllllllll}220 & 210 & 200 & 190 & 180 & 170 & 160 & 150 & 140 & 130 & 120 & 110 & 100 & 90 & 80 & 70 & 60 & 50 & 40 & 30 & 20 & 10 & 0 & -10\end{array}$ 

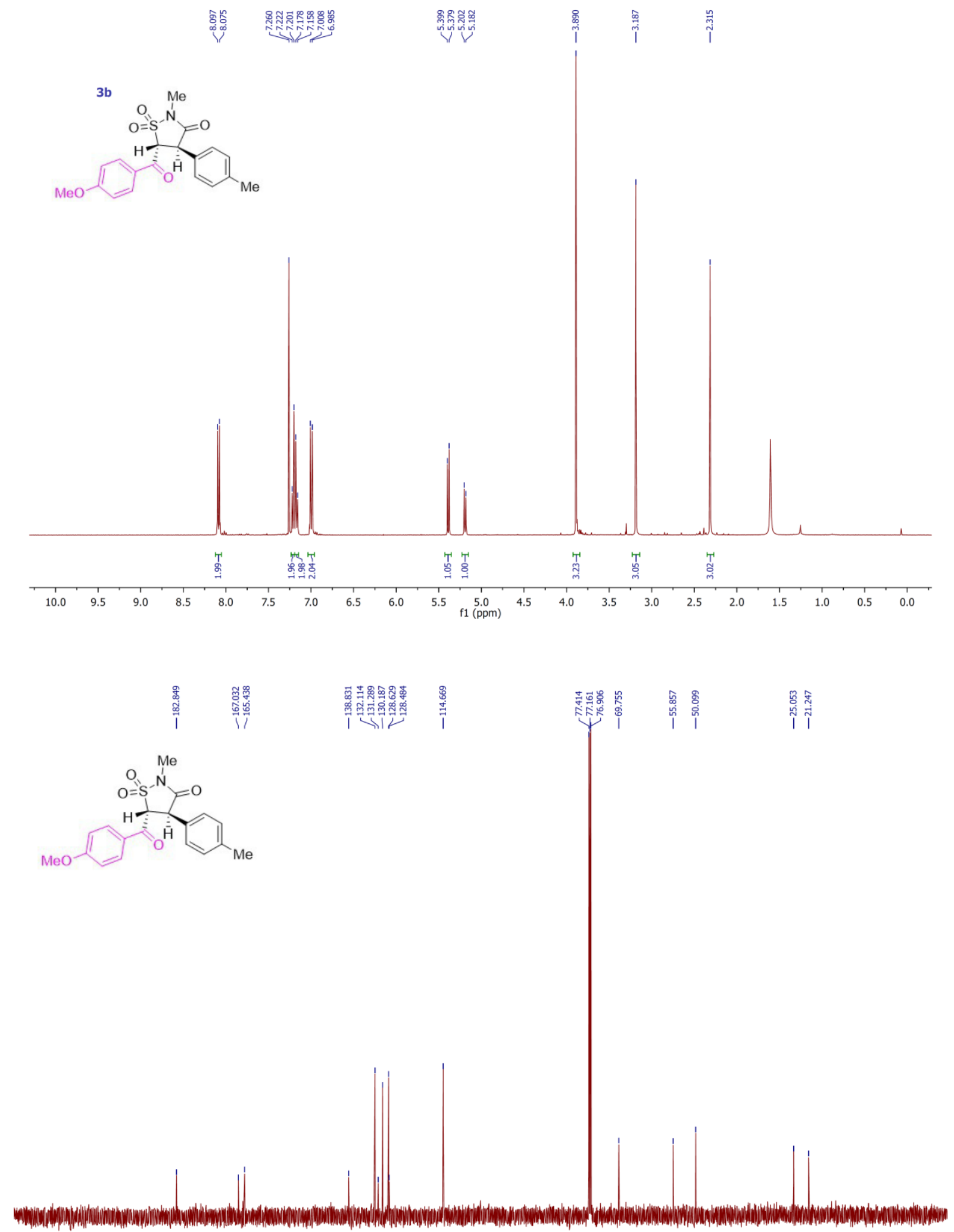

$\begin{array}{llllllllllllllllllllllllllllllllll}220 & 210 & 200 & 190 & 180 & 170 & 160 & 150 & 140 & 130 & 120 & 110 & 100 & 90 & 80 & 70 & 60 & 50 & 40 & 30 & 20 & 10 & 0 & -10\end{array}$ 

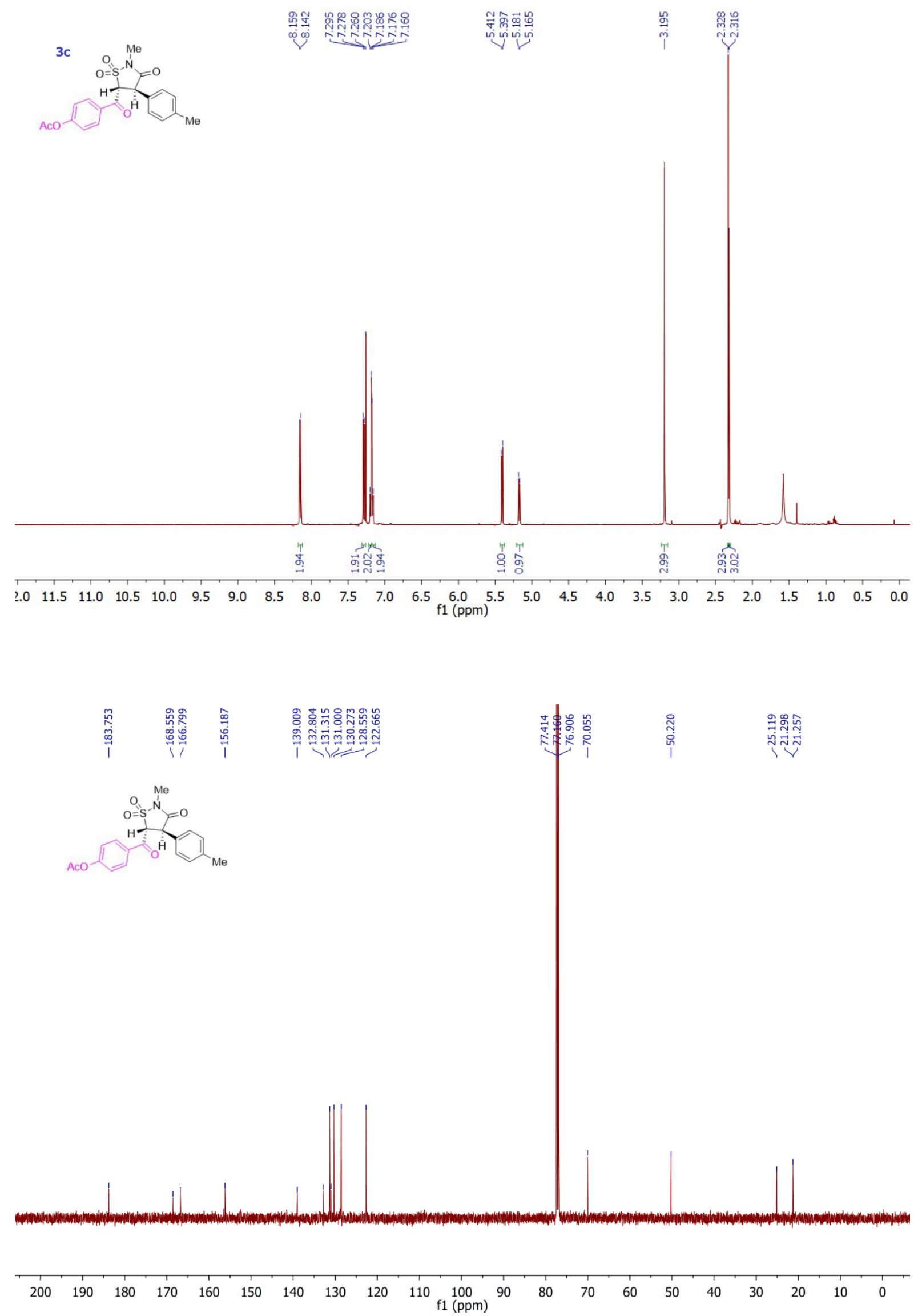

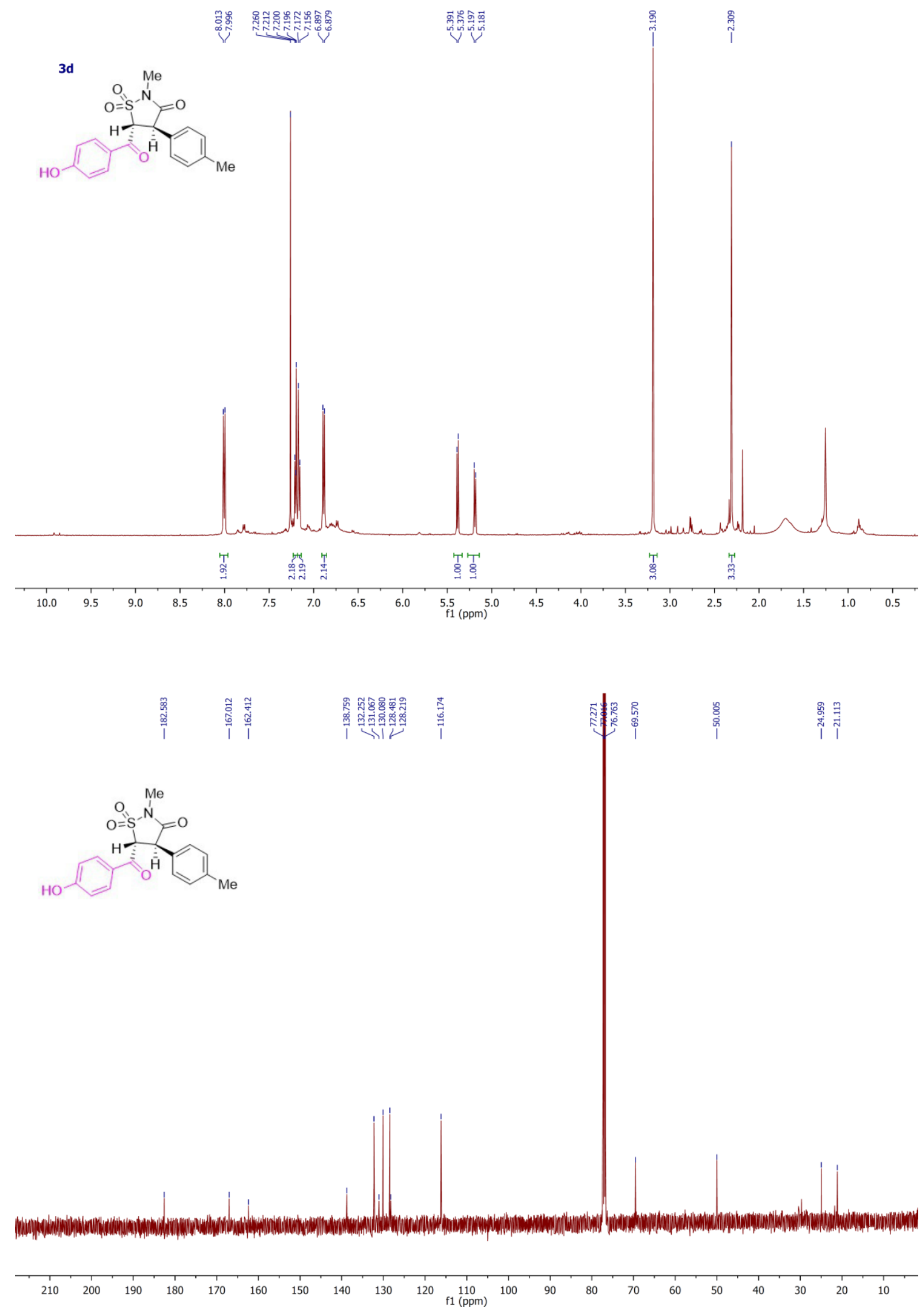

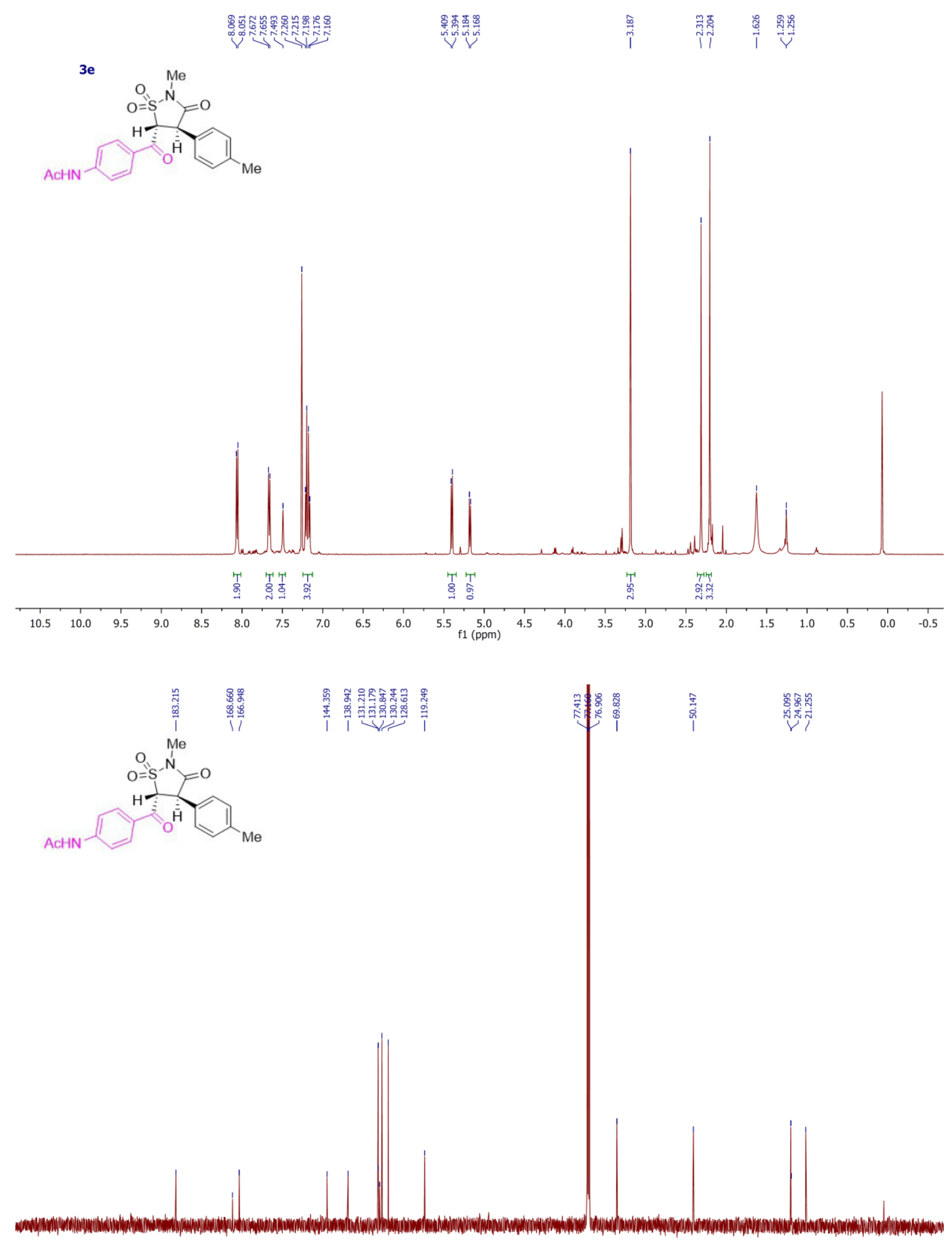

$\begin{array}{rrrrrrrrrrrrrrrrrrrrrrrrrrrrr}1 & 1 \\ 220 & 210 & 200 & 190 & 180 & 170 & 160 & 150 & 140 & 130 & 120 & 110 & 100 & 90 & 80 & 70 & 60 & 50 & 40 & 30 & 20 & 10 & 0 & -10\end{array}$ 

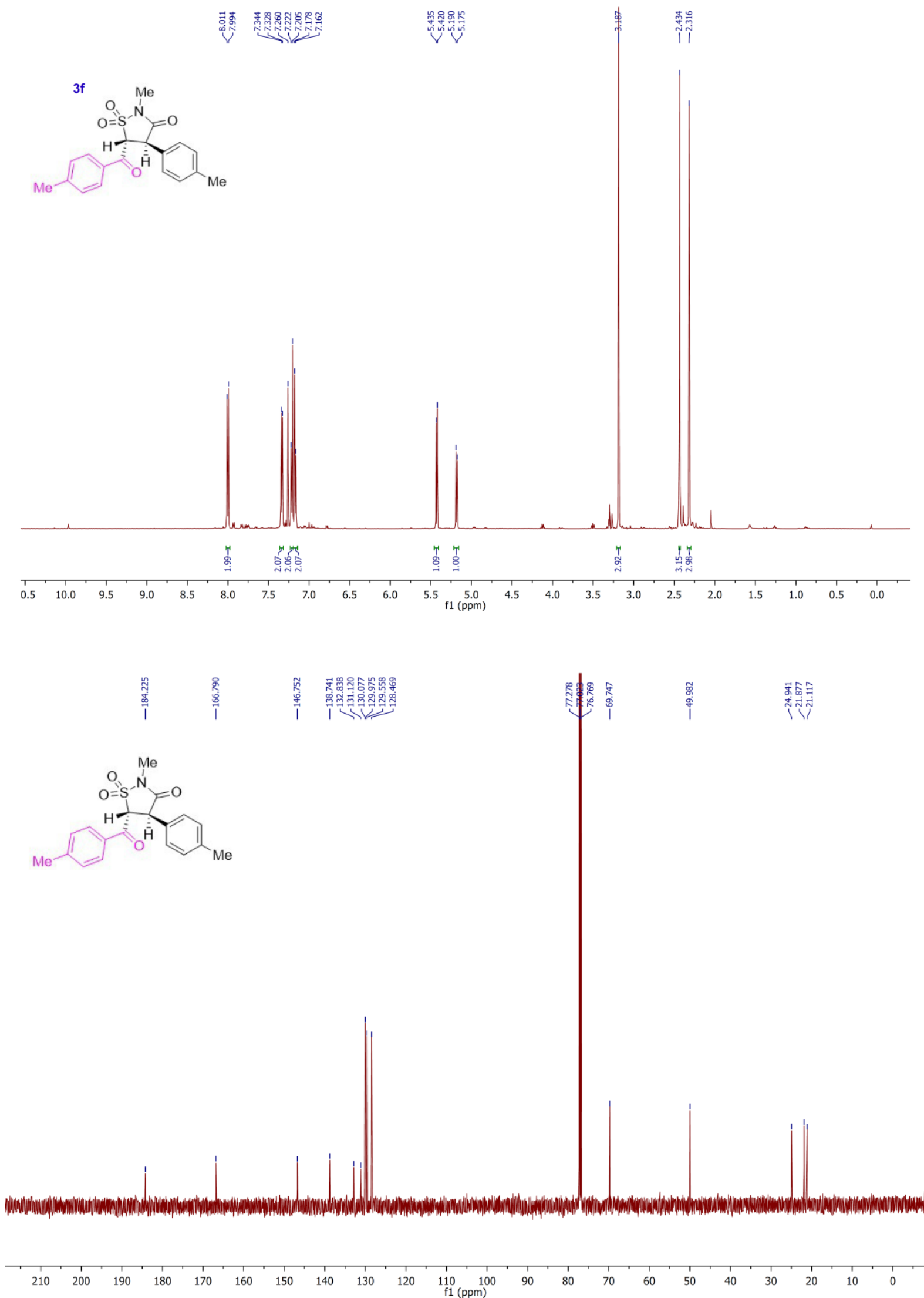

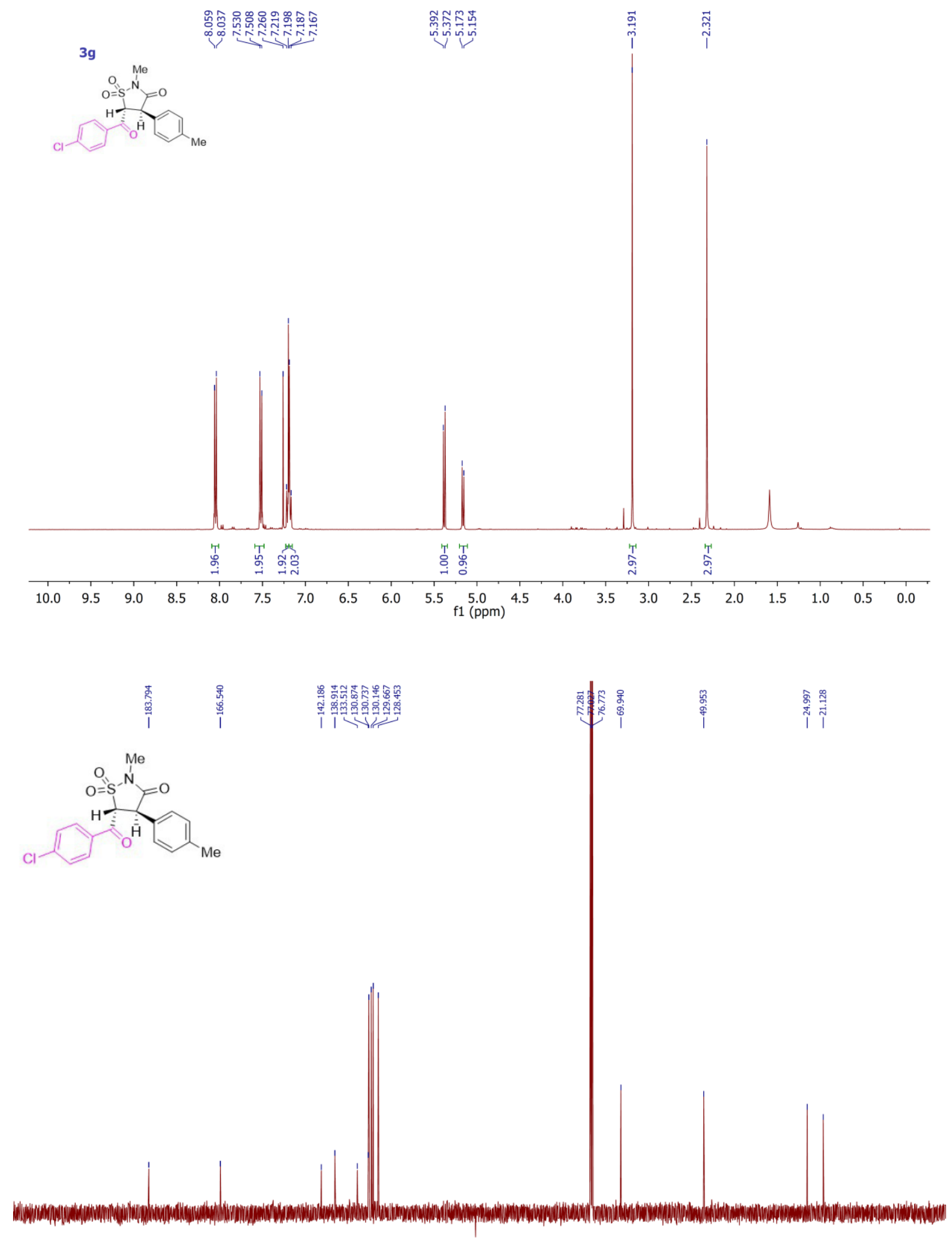

$\begin{array}{llllllllllllllllllllllllllllll}210 & 200 & 190 & 180 & 170 & 160 & 150 & 140 & 130 & 120 & 110 & 100 & 90 & 80 & 70 & 60 & 50 & 40 & 30 & 20 & 10 & 0\end{array}$ 

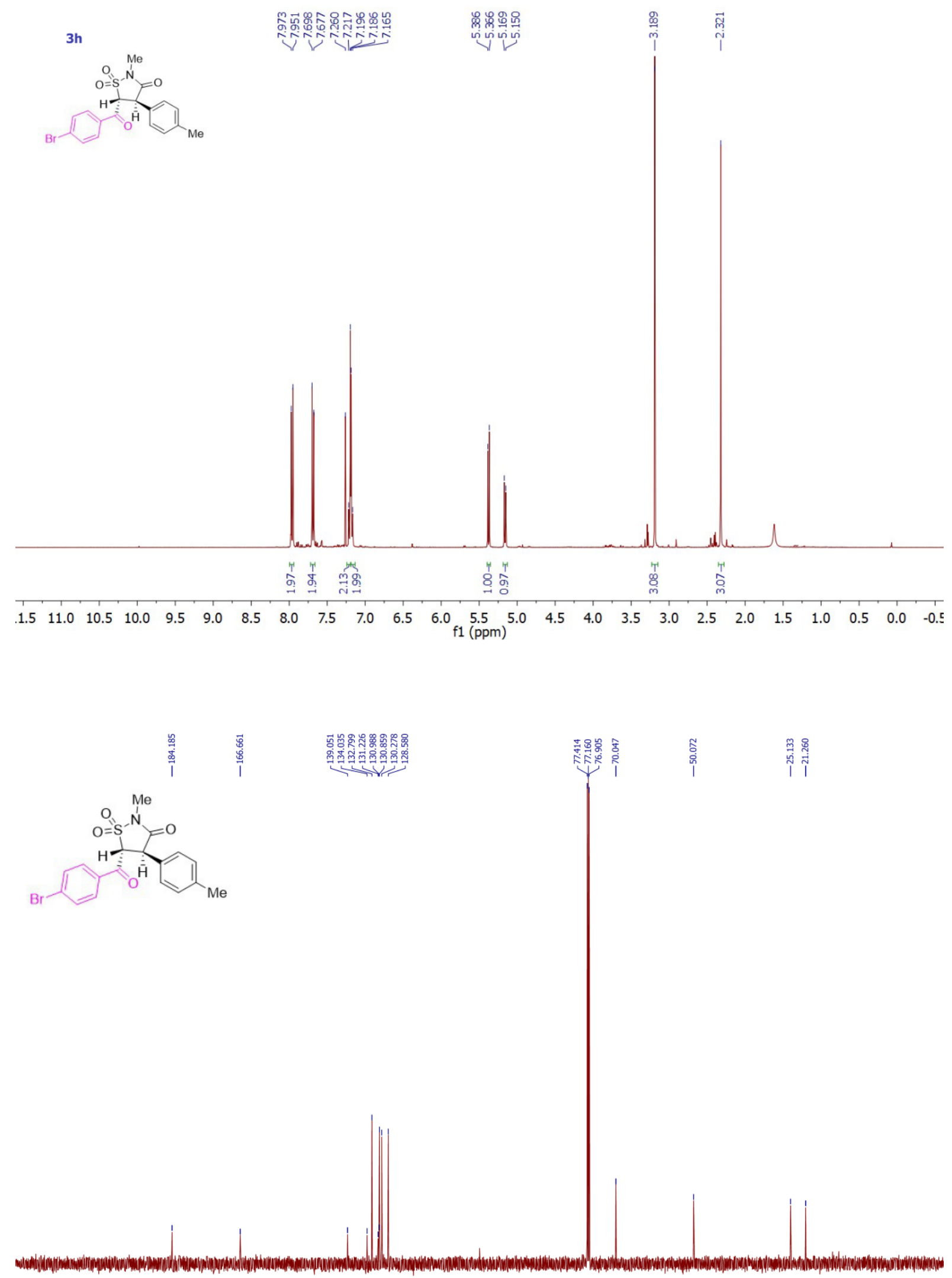

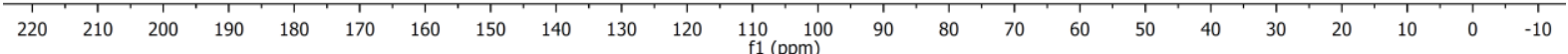




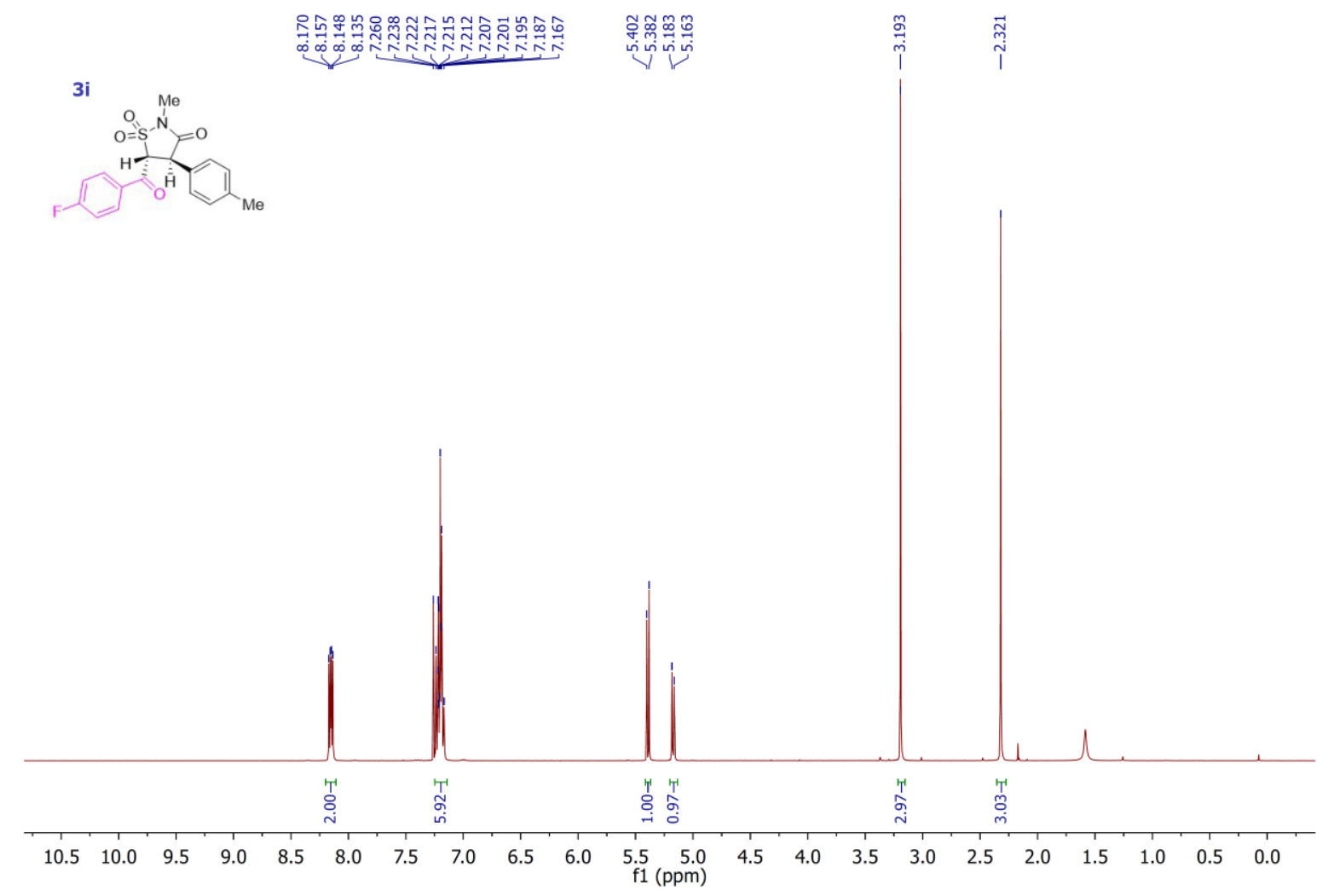

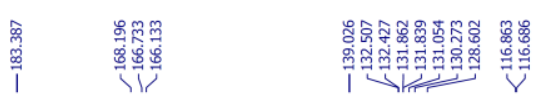

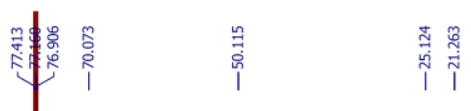
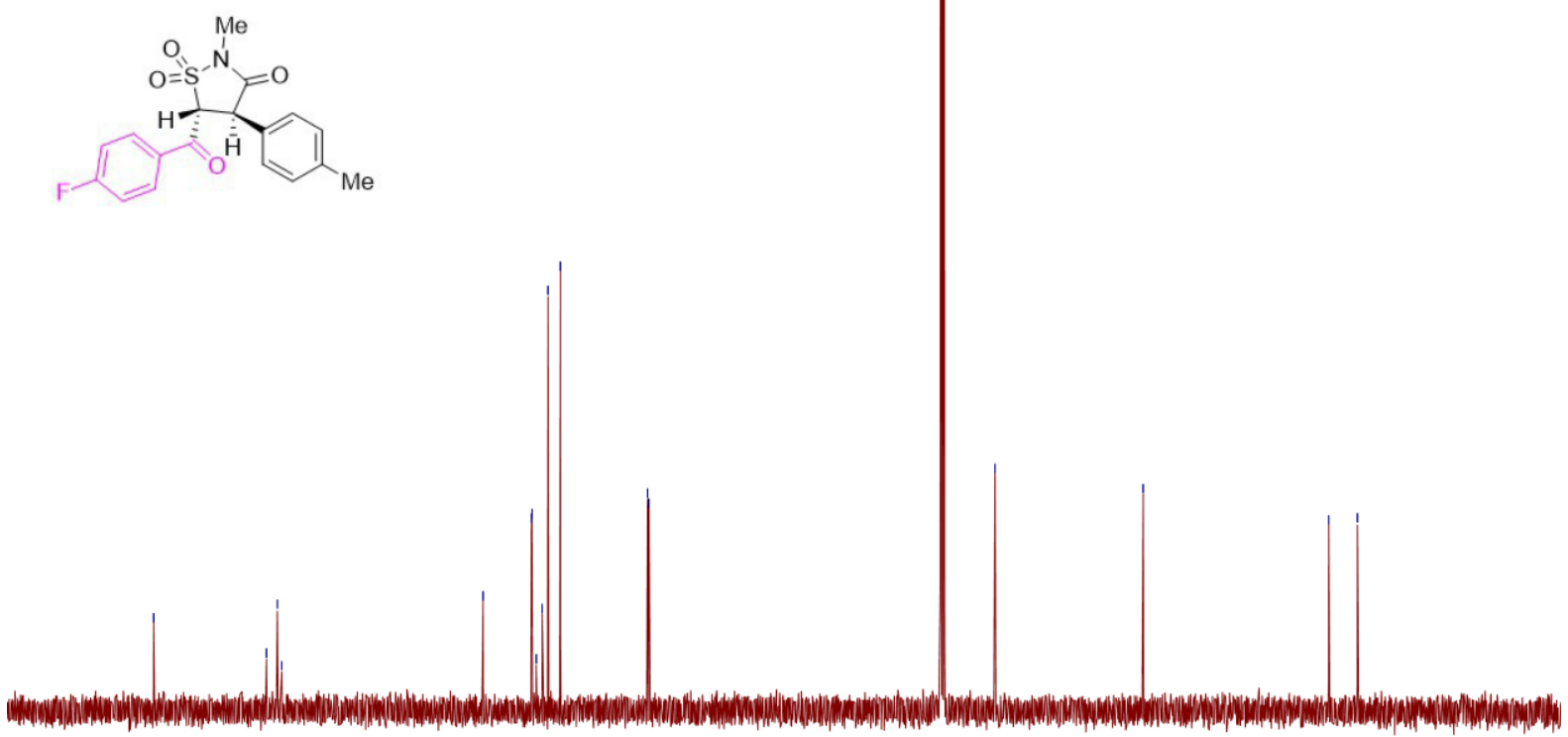

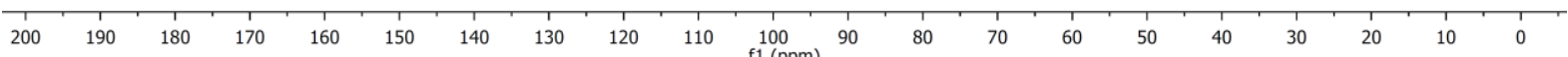


${ }^{19} \mathrm{~F} \mathrm{NMR}\left(376 \mathrm{MHz}, \mathrm{CDCl}_{3}\right) \delta$ - 100.49.
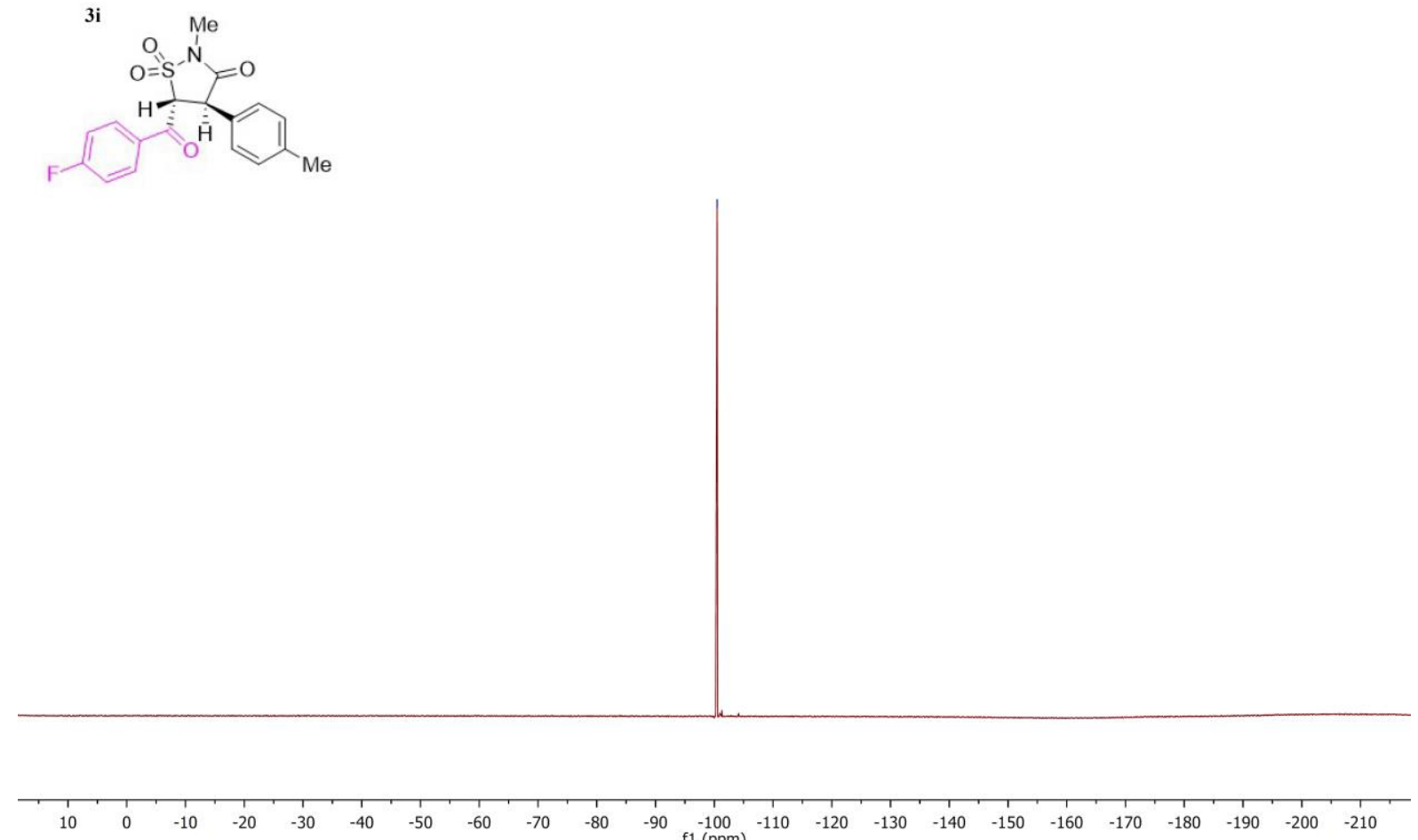

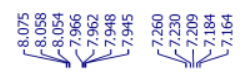

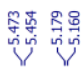
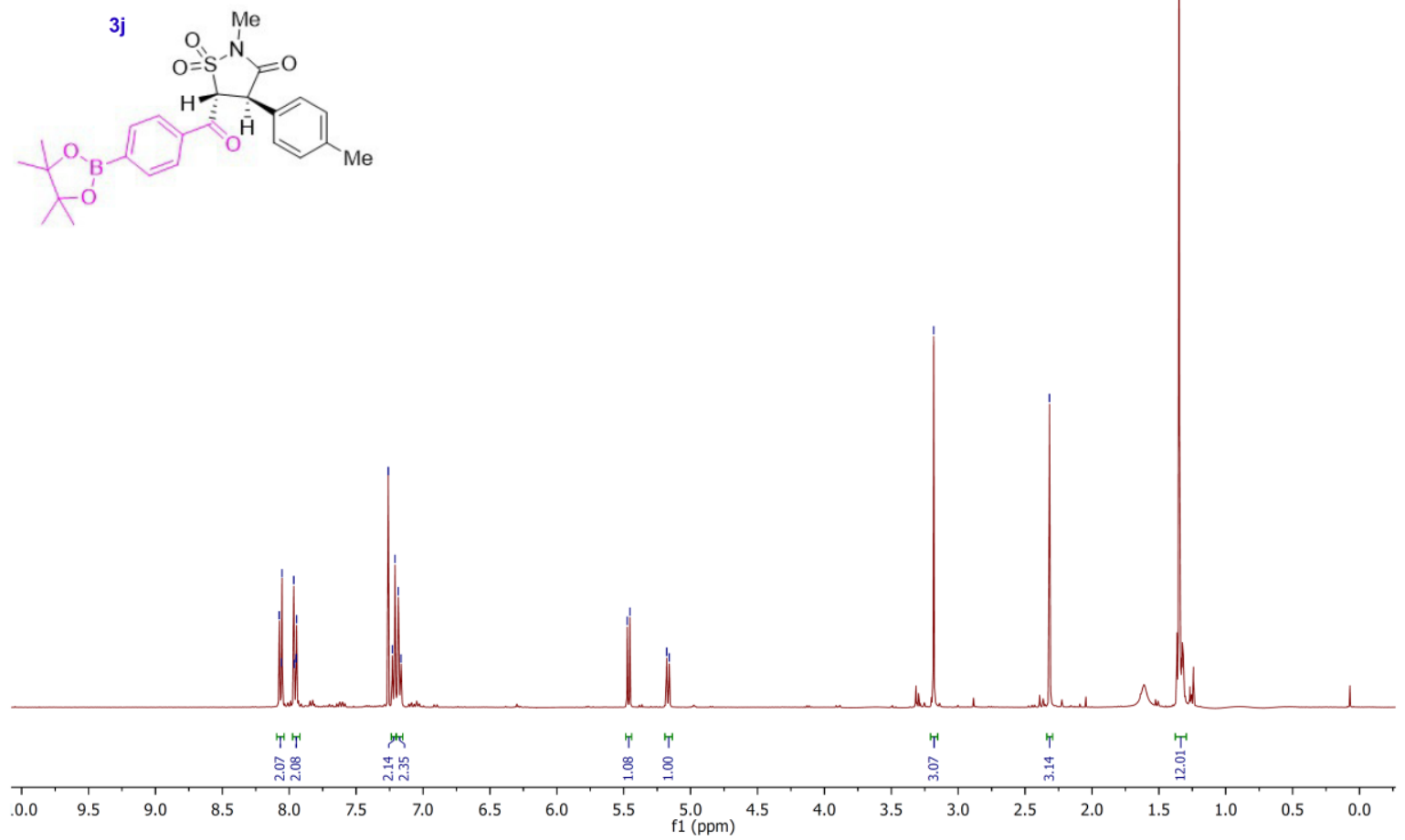

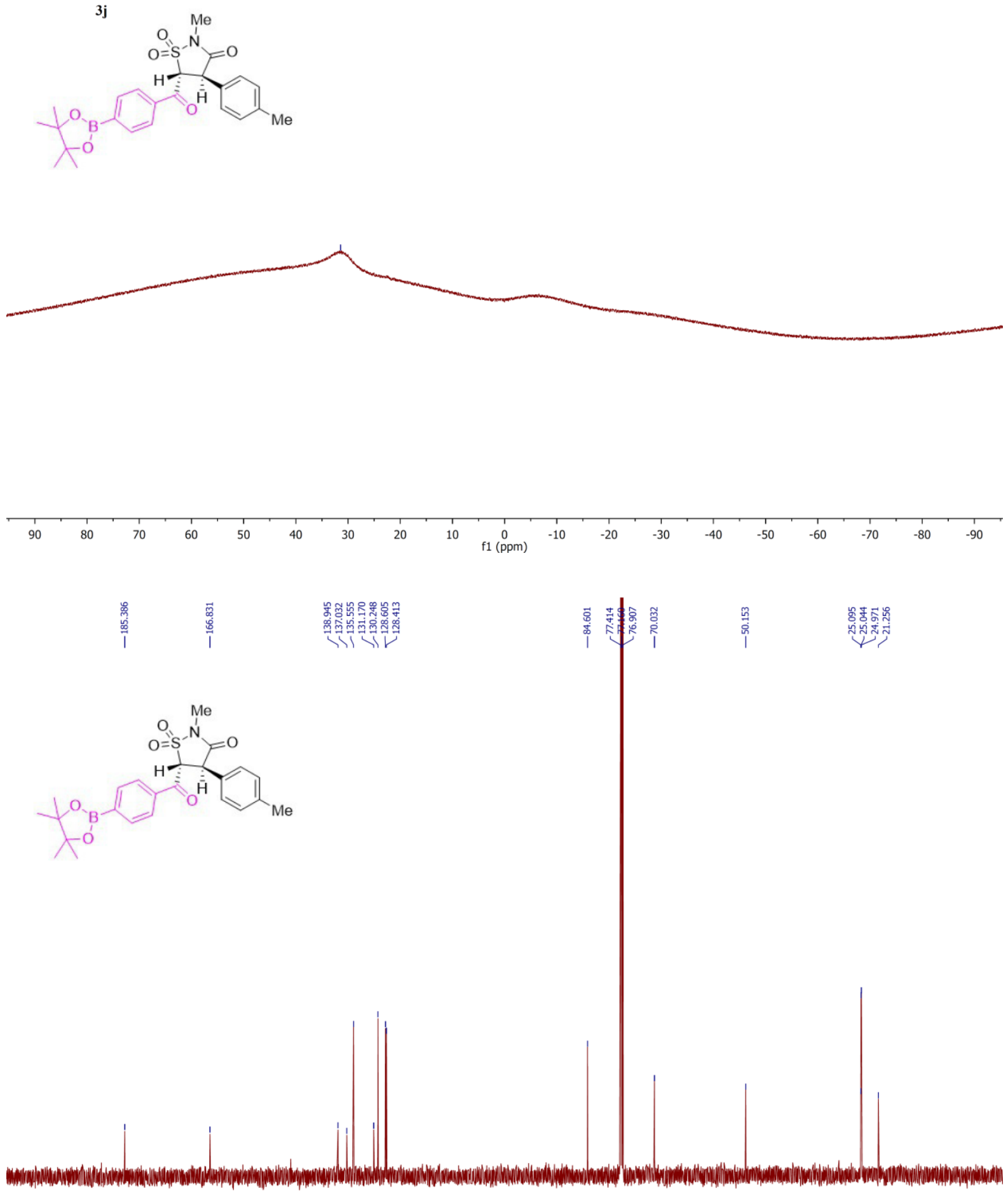

\begin{tabular}{lllllllllllllllllllllllll}
\hline 10 & 200 & 190 & 180 & 170 & 160 & 150 & 140 & 130 & 120 & 110 & 100 & 90 & 80 & 70 & 60 & 50 & 40 & 30 & 20 & 10 & 0
\end{tabular} 

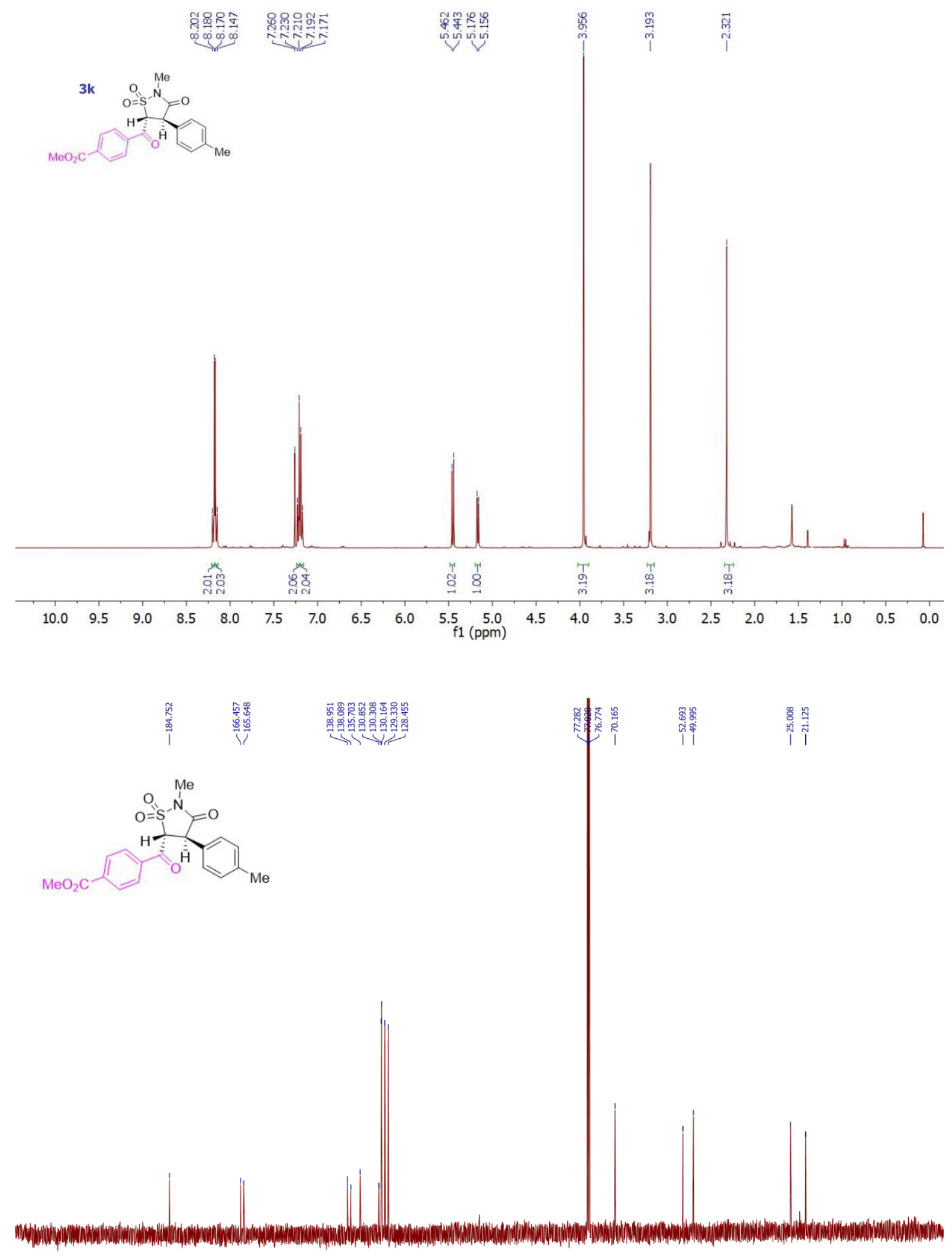

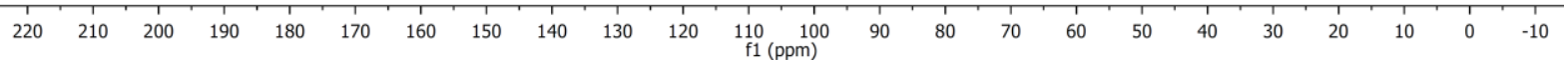




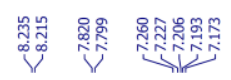

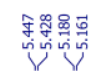
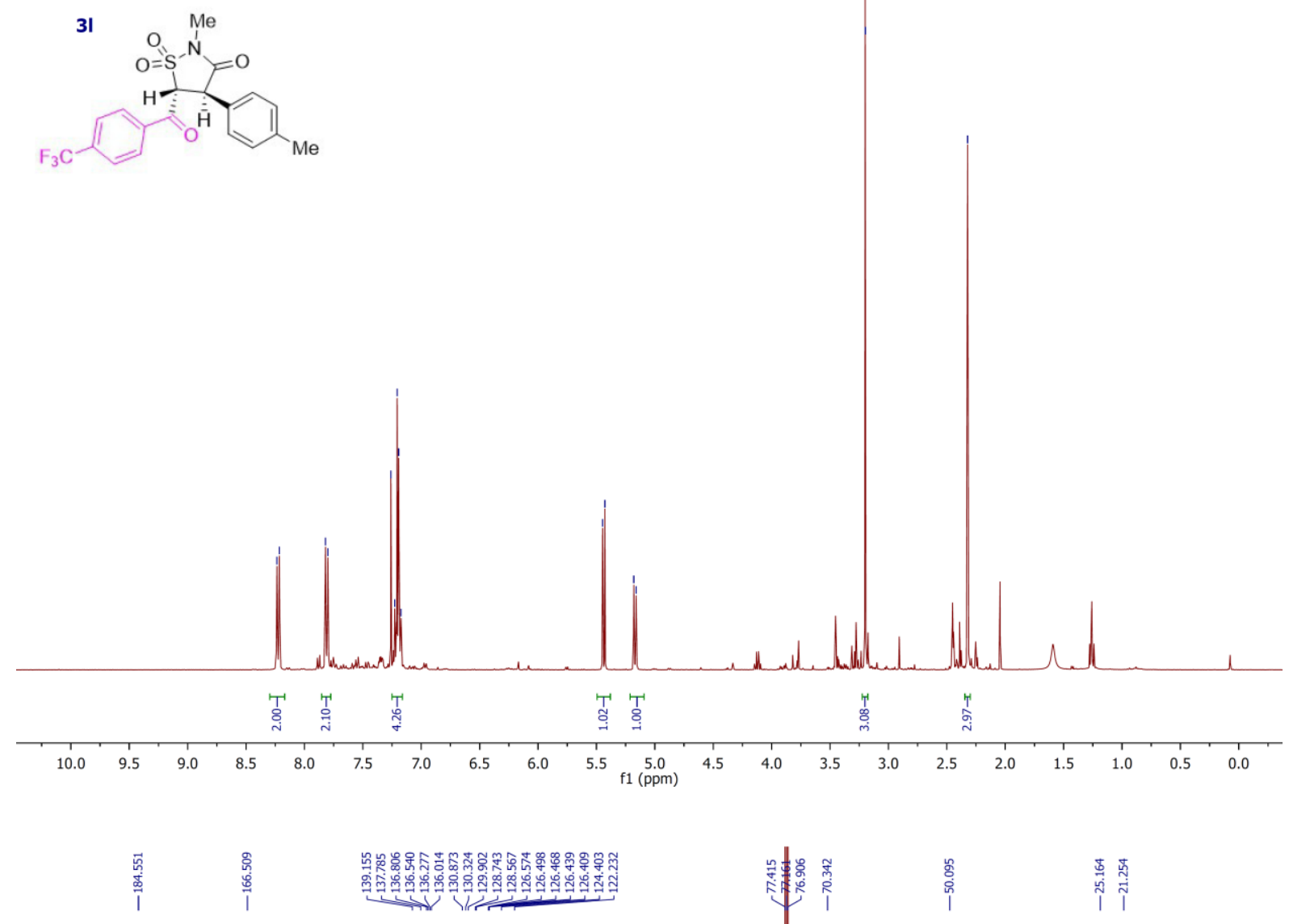

志苟
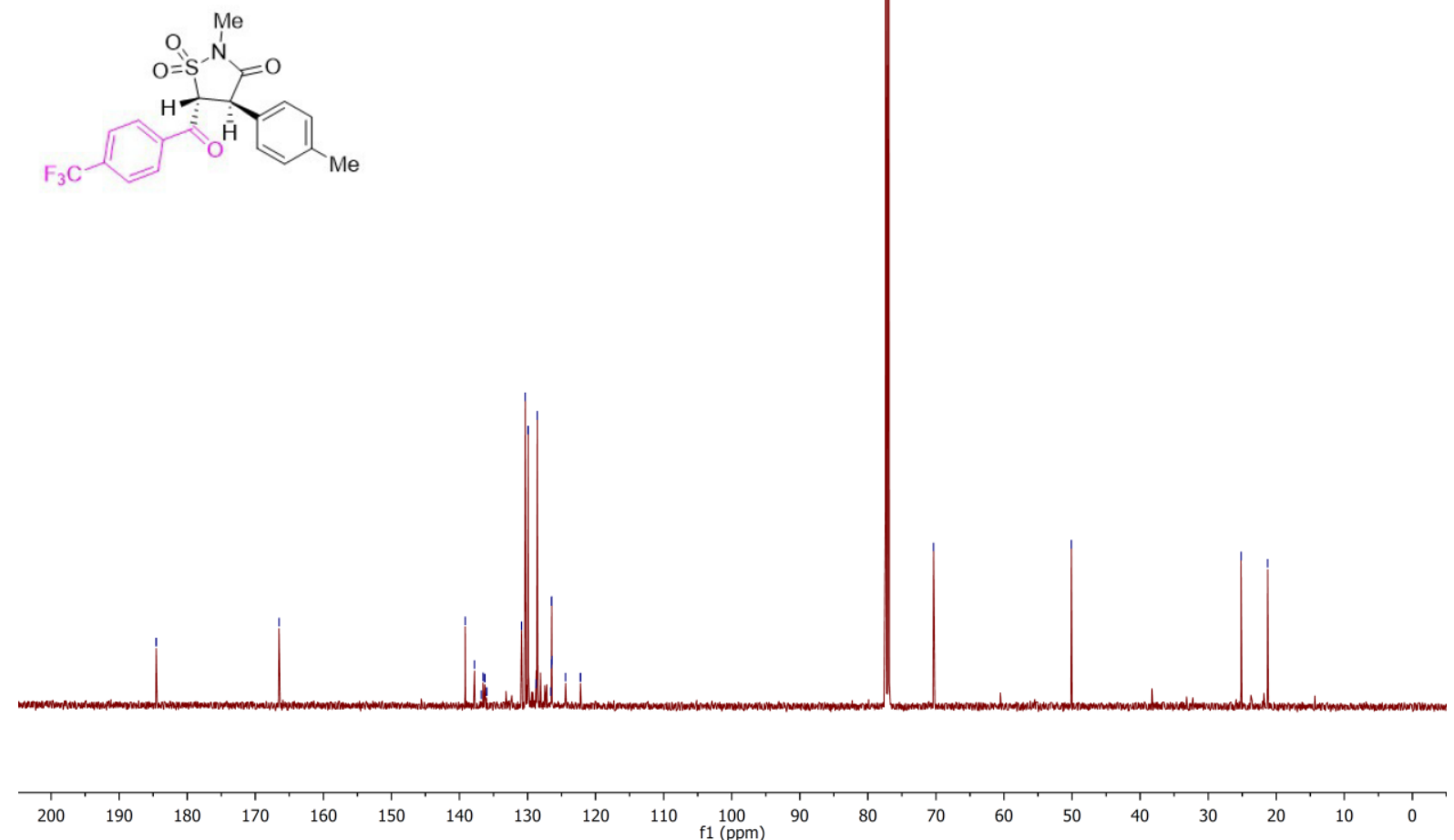
${ }^{19} \mathrm{~F} \mathrm{NMR}\left(377 \mathrm{MHz}, \mathrm{CDCl}_{3}\right) \delta-63.45$.

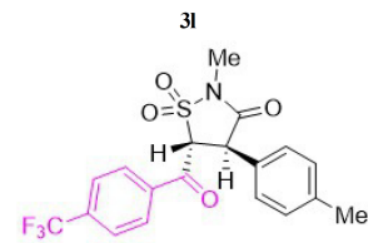

\begin{tabular}{rlllllllllllllllllllllll}
\hline 10 & 0 & -10 & -20 & -30 & -40 & -50 & -60 & -70 & -80 & -90 & -100 & -110 & -120 & -130 & -140 & -150 & -160 & -170 & -180 & -190 & -200 & -210
\end{tabular}

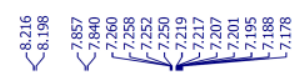

琴琴琴
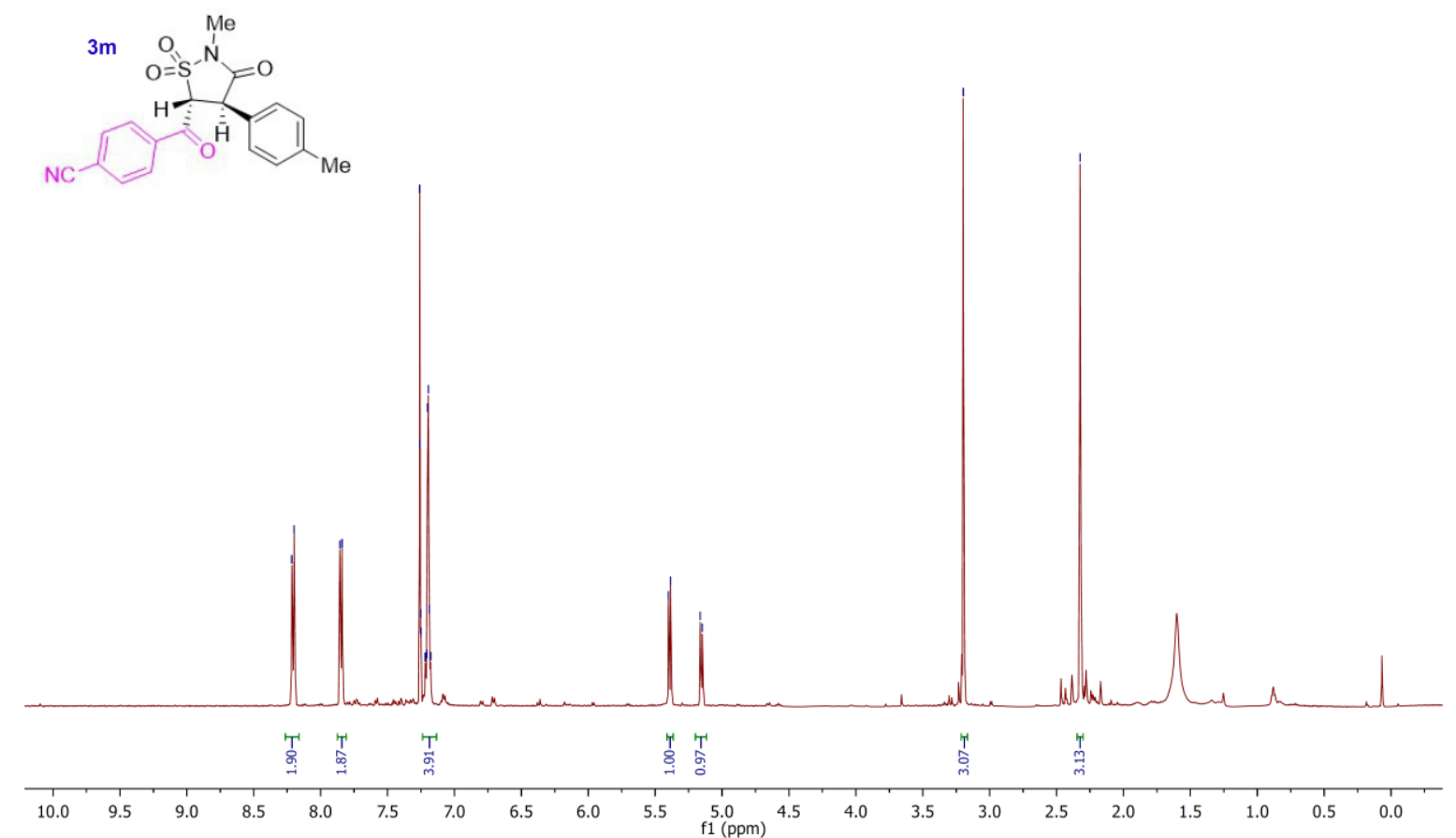

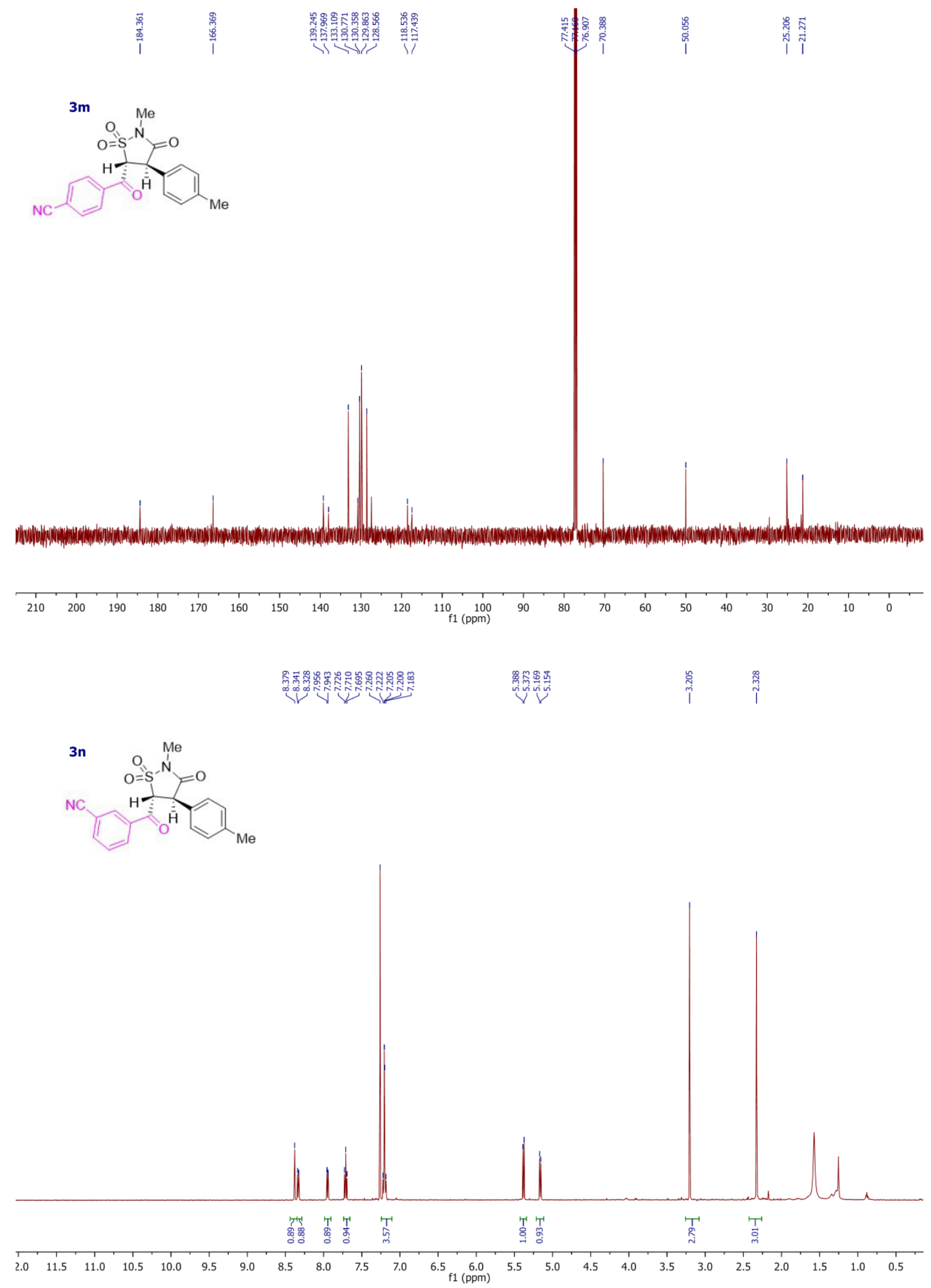


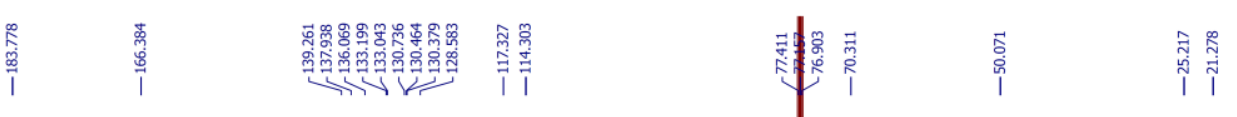
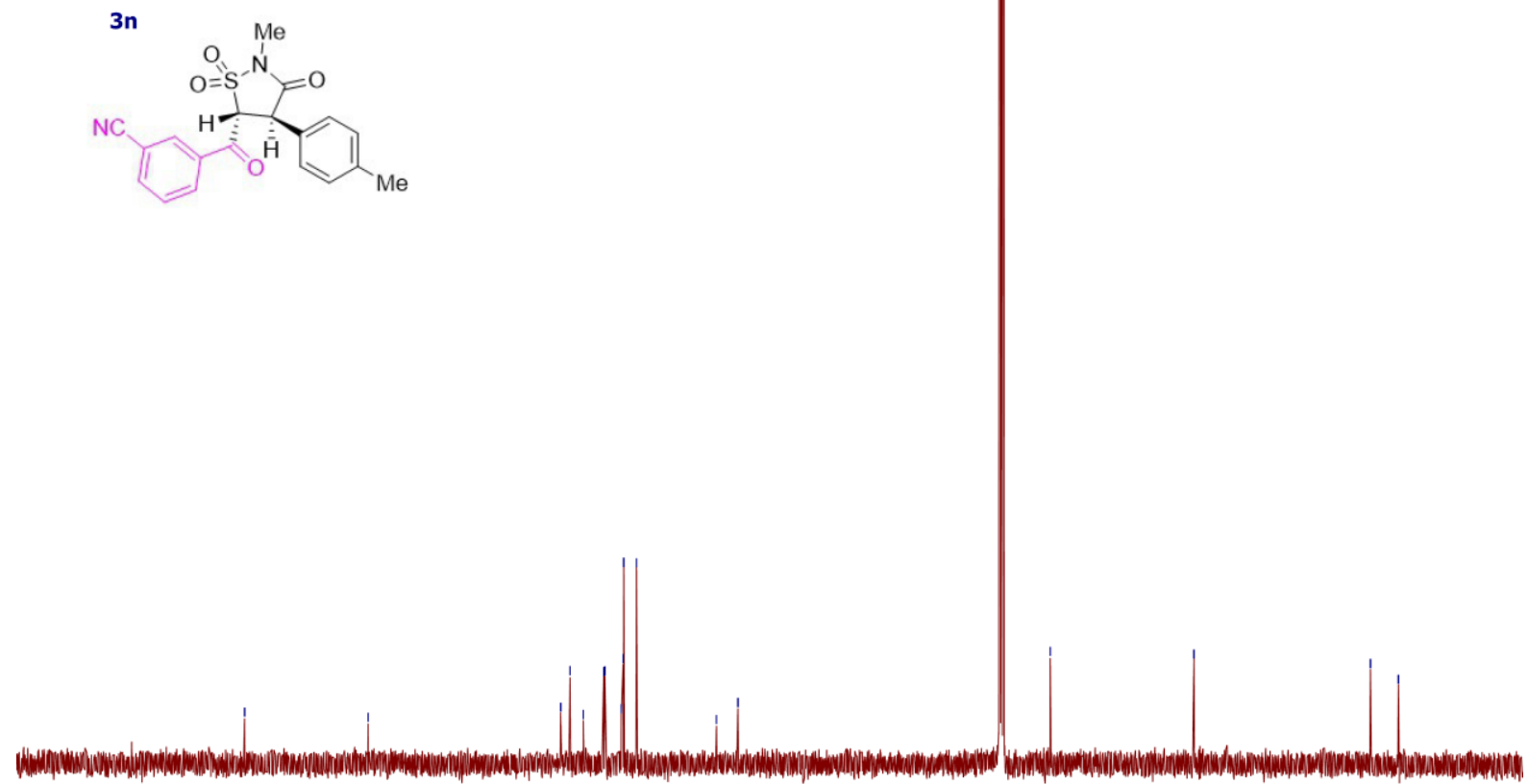

\begin{tabular}{|c|c|c|c|c|c|c|c|c|c|c|c|c|c|c|c|c|c|c|}
\hline 210 & 200 & 190 & 180 & 170 & 160 & 150 & 140 & 130 & 120 & 110 & 100 & 90 & 80 & 70 & 60 & 50 & 40 & 30 \\
\hline
\end{tabular}

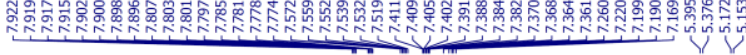
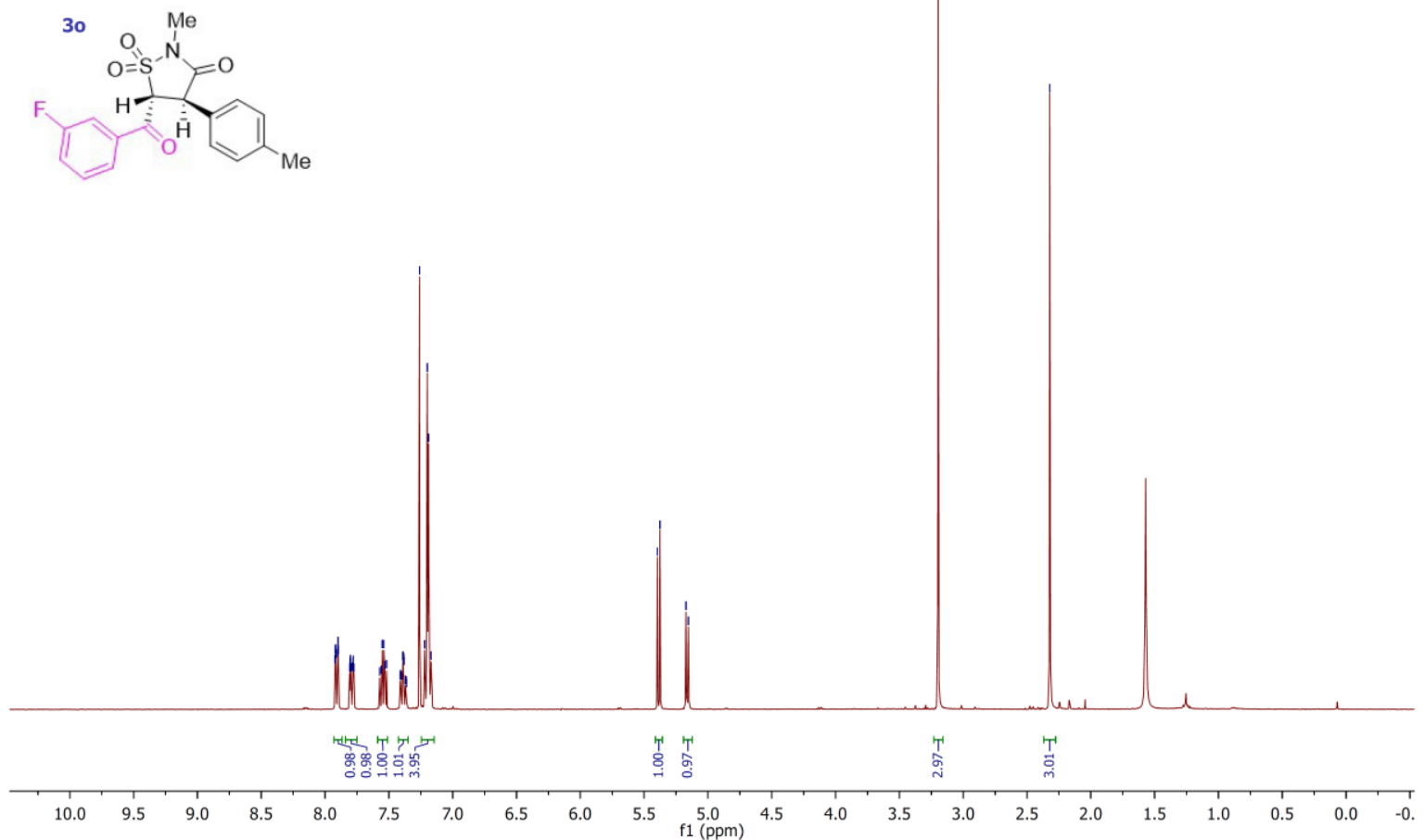
30<smiles>Cc1ccc([C@H]2C(=O)N(C)S(=O)(=O)[C@@H]2C(=O)c2cccc(F)c2)cc1</smiles>
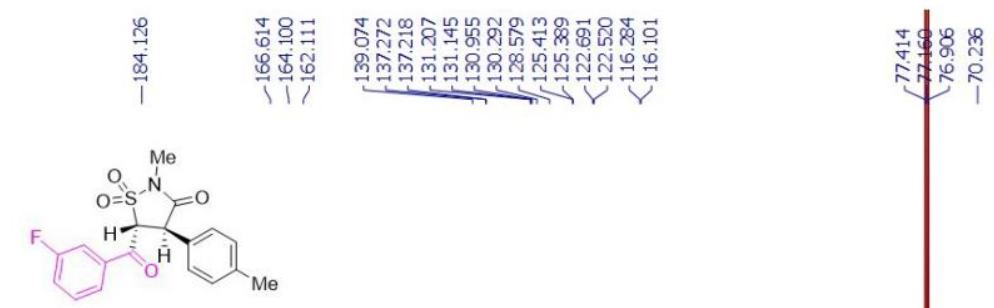

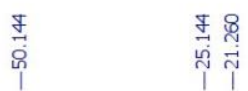

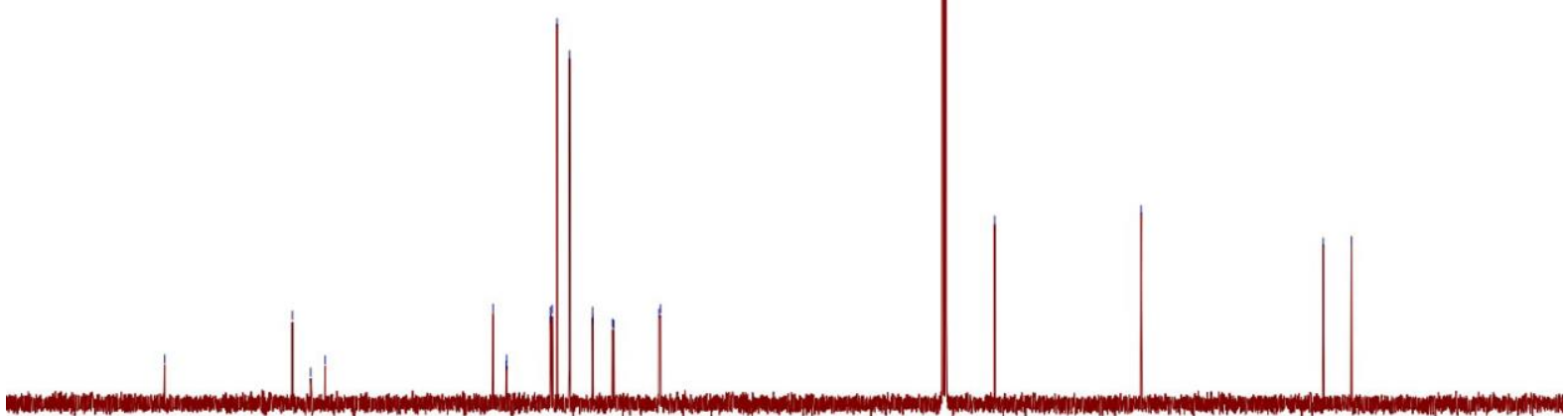

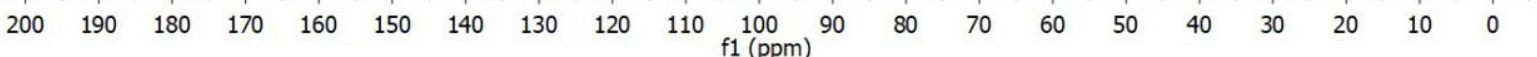



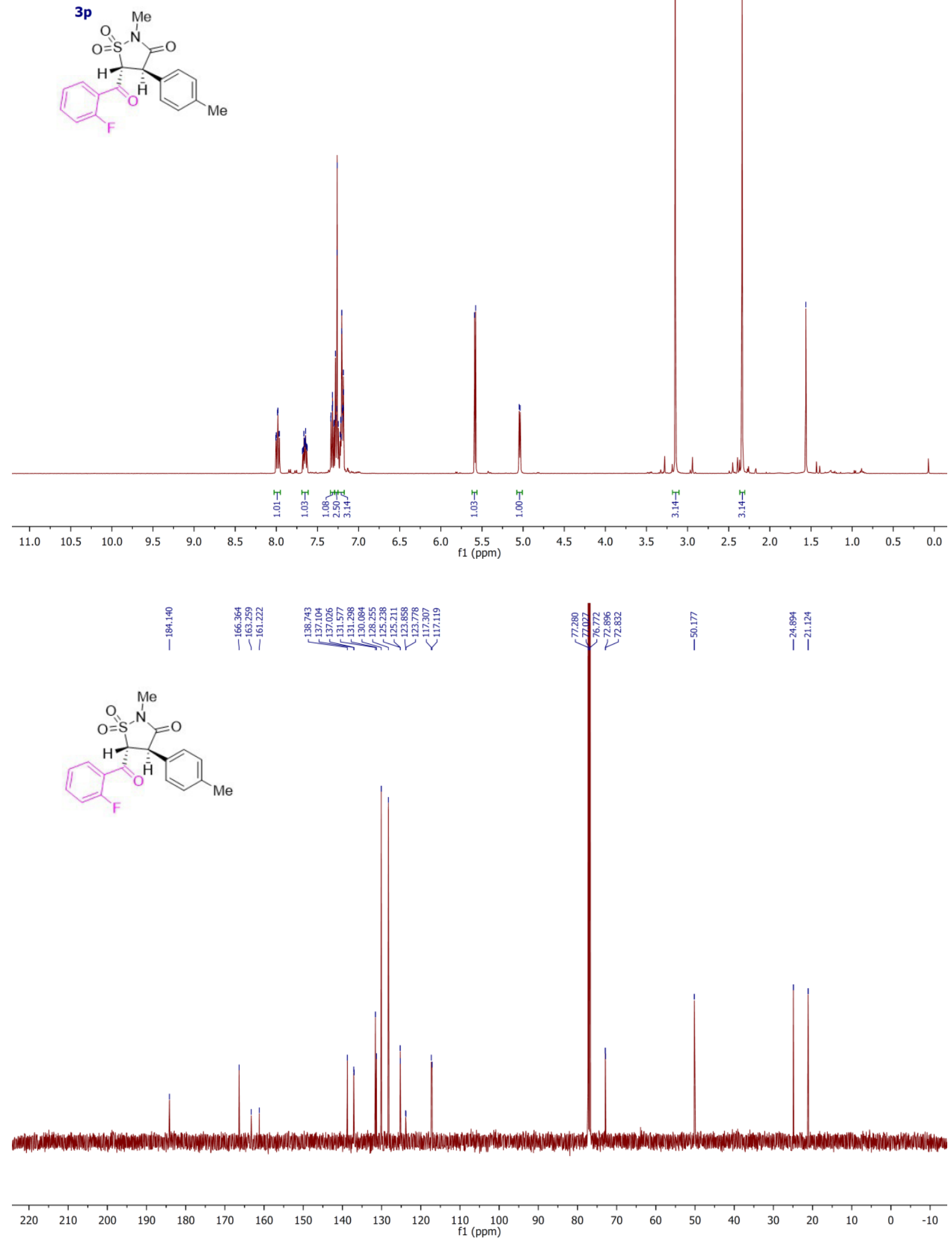
${ }^{19} \mathrm{~F}$ NMR $\left(377 \mathrm{MHz}, \mathrm{CDCl}_{3}\right) \delta-108.25$

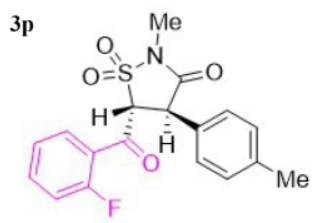

$\begin{array}{llllllllllllllllllllllll}1 & 10 & 0 & -10 & -20 & -30 & -40 & -50 & -60 & -70 & -80 & -90 & -100 & -110 & -120 & -130 & -140 & -150 & -160 & -170 & -180 & -190 & -200 & -210\end{array}$

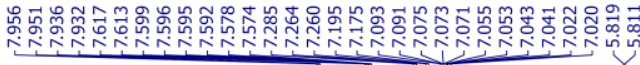

$3 q$
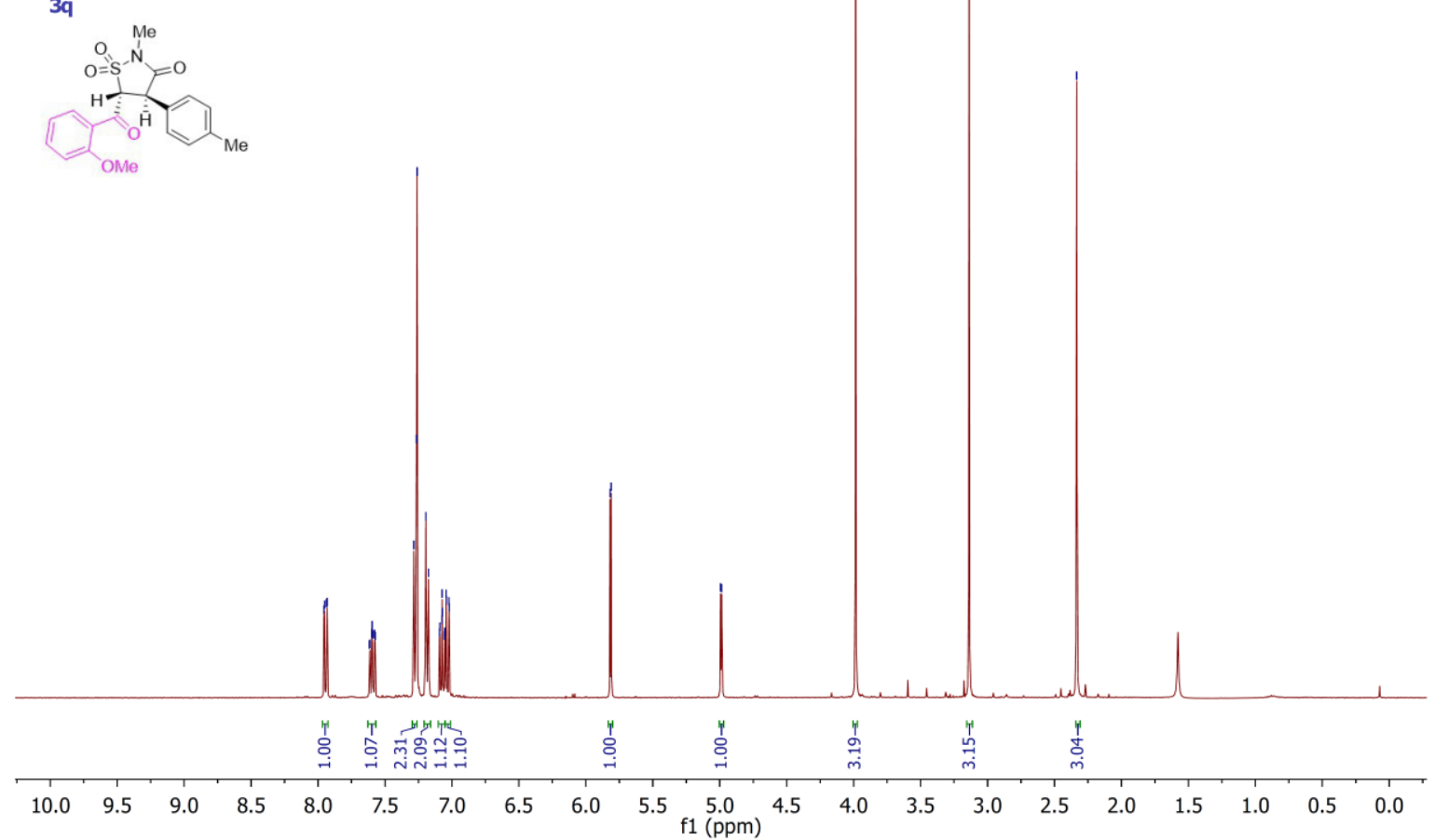

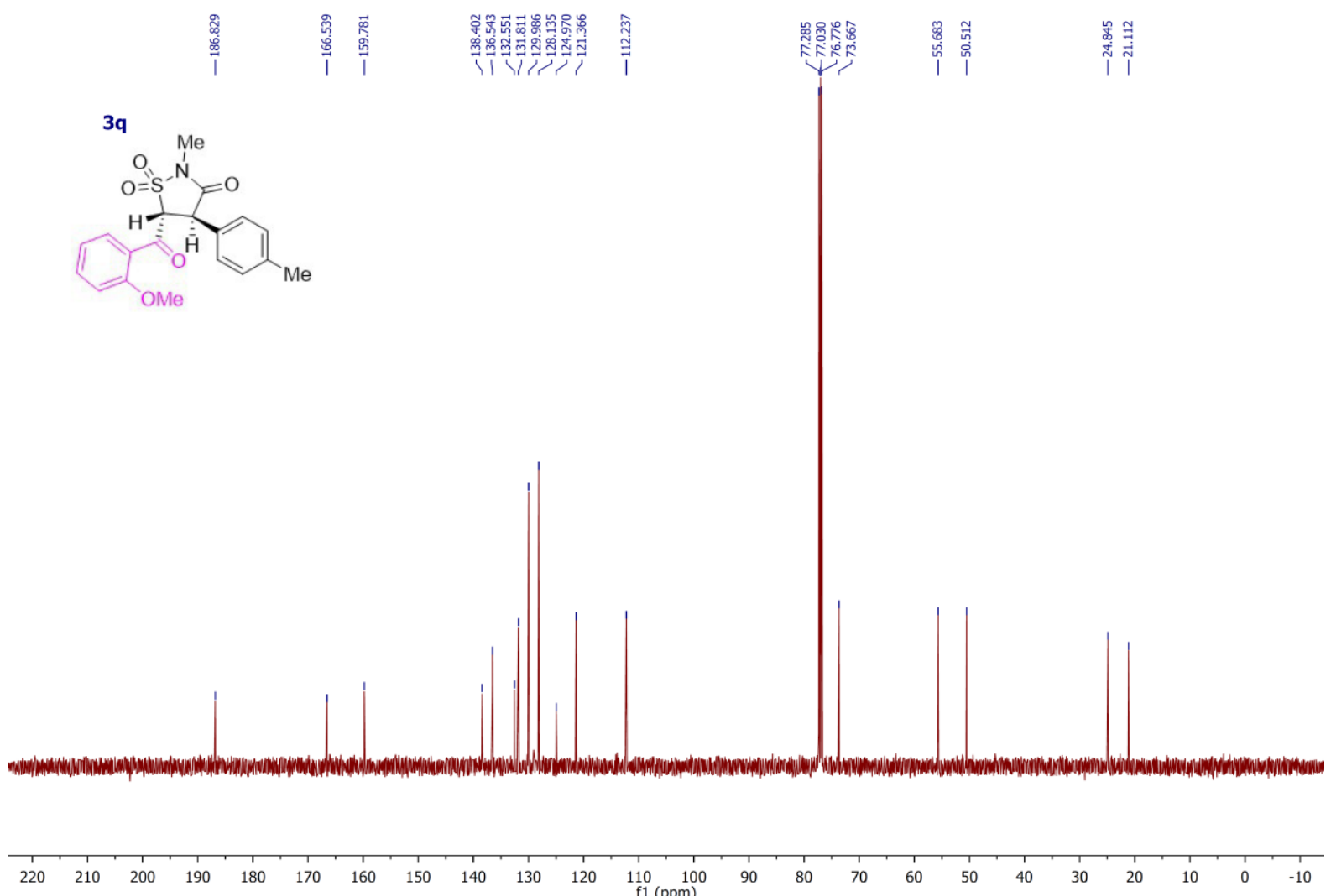

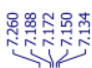
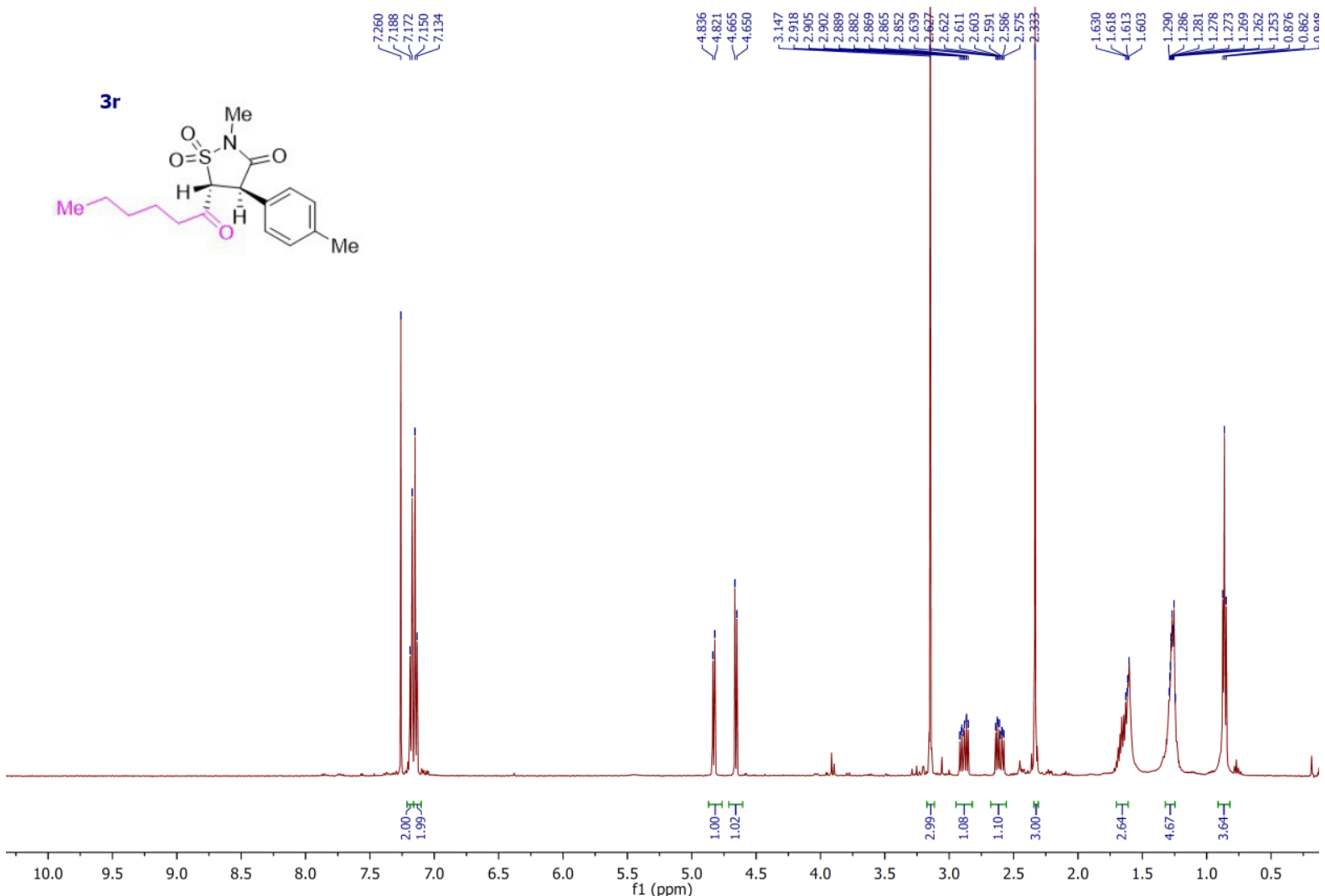

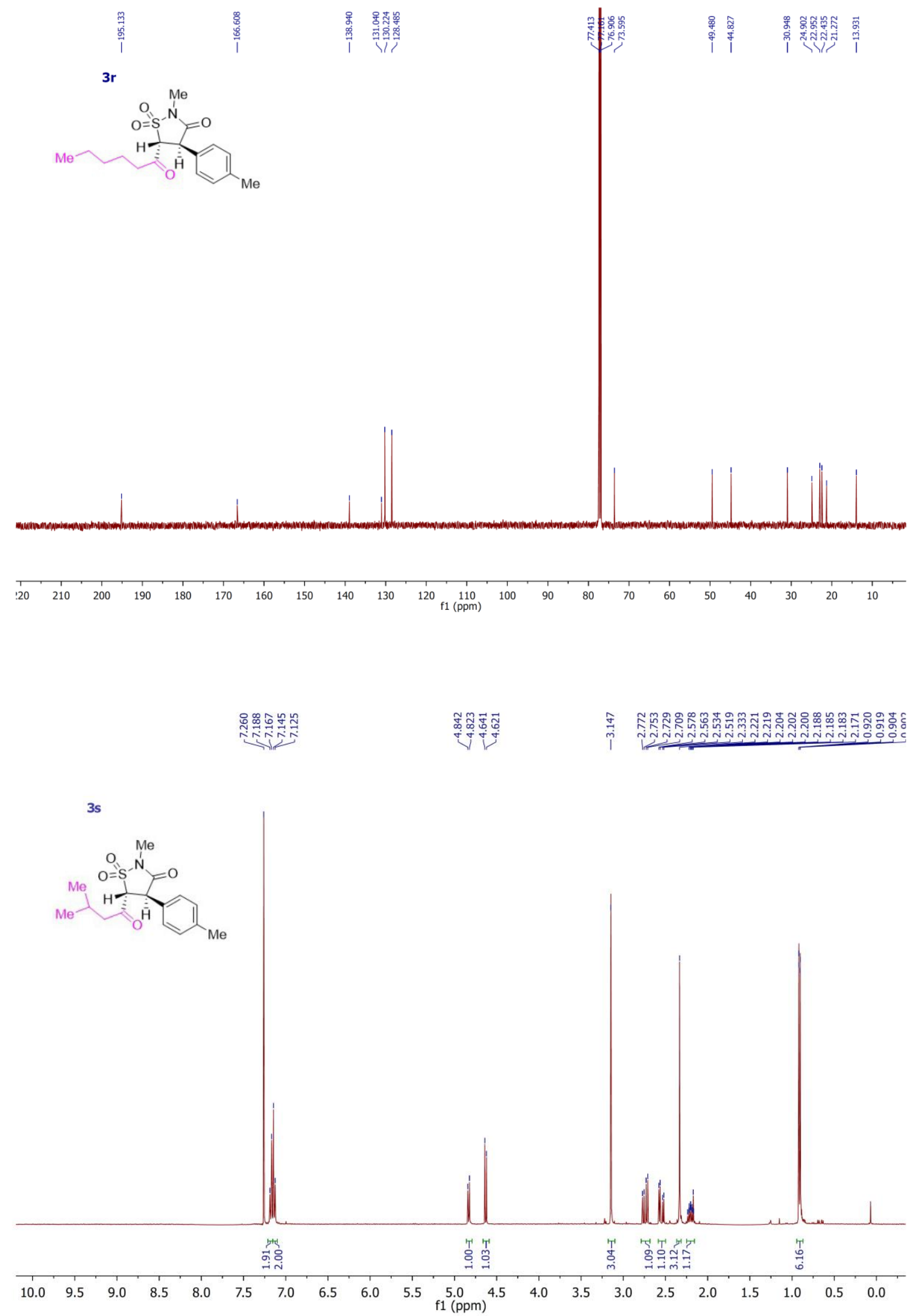

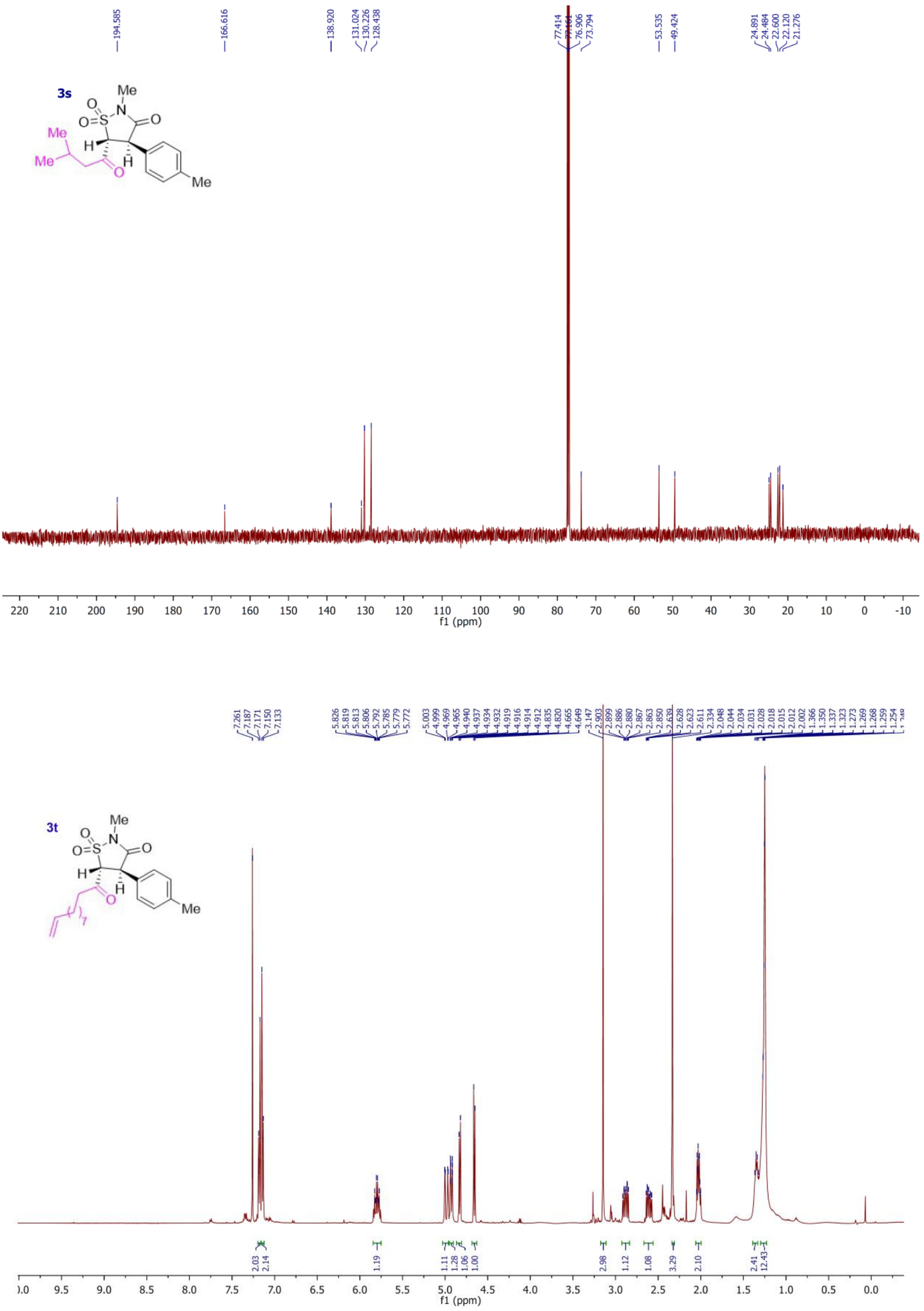

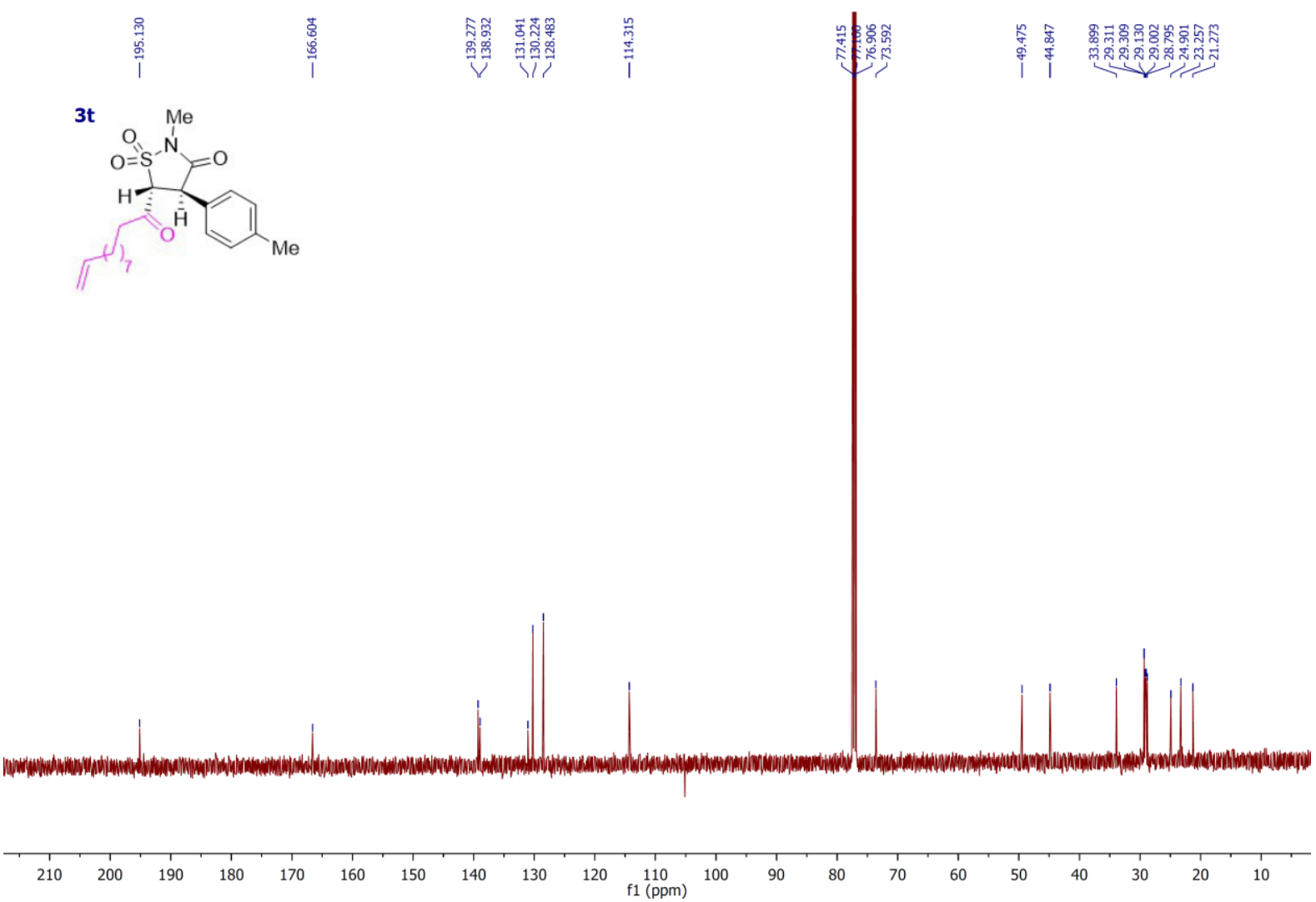

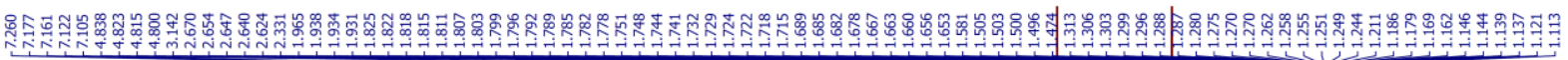
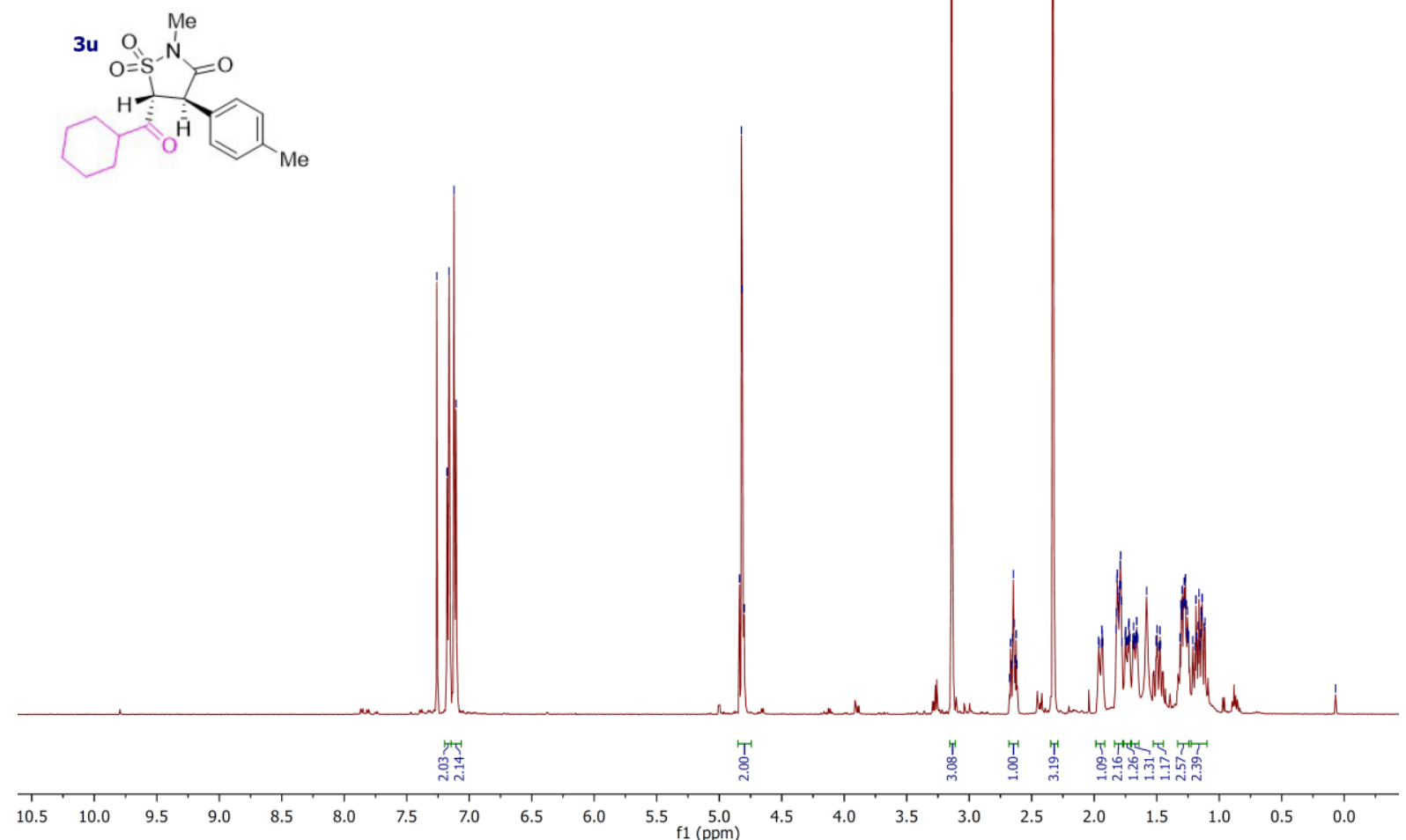


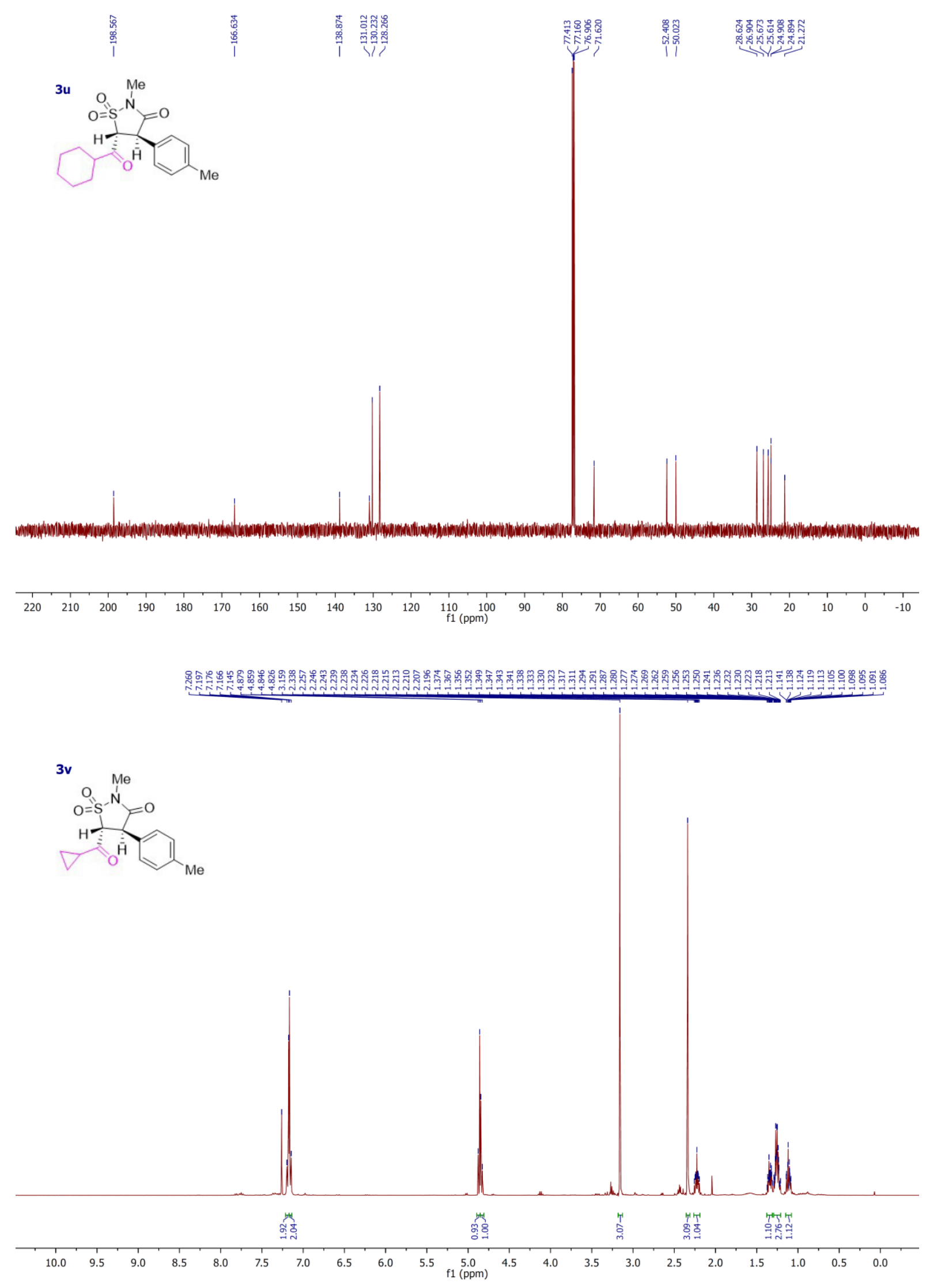



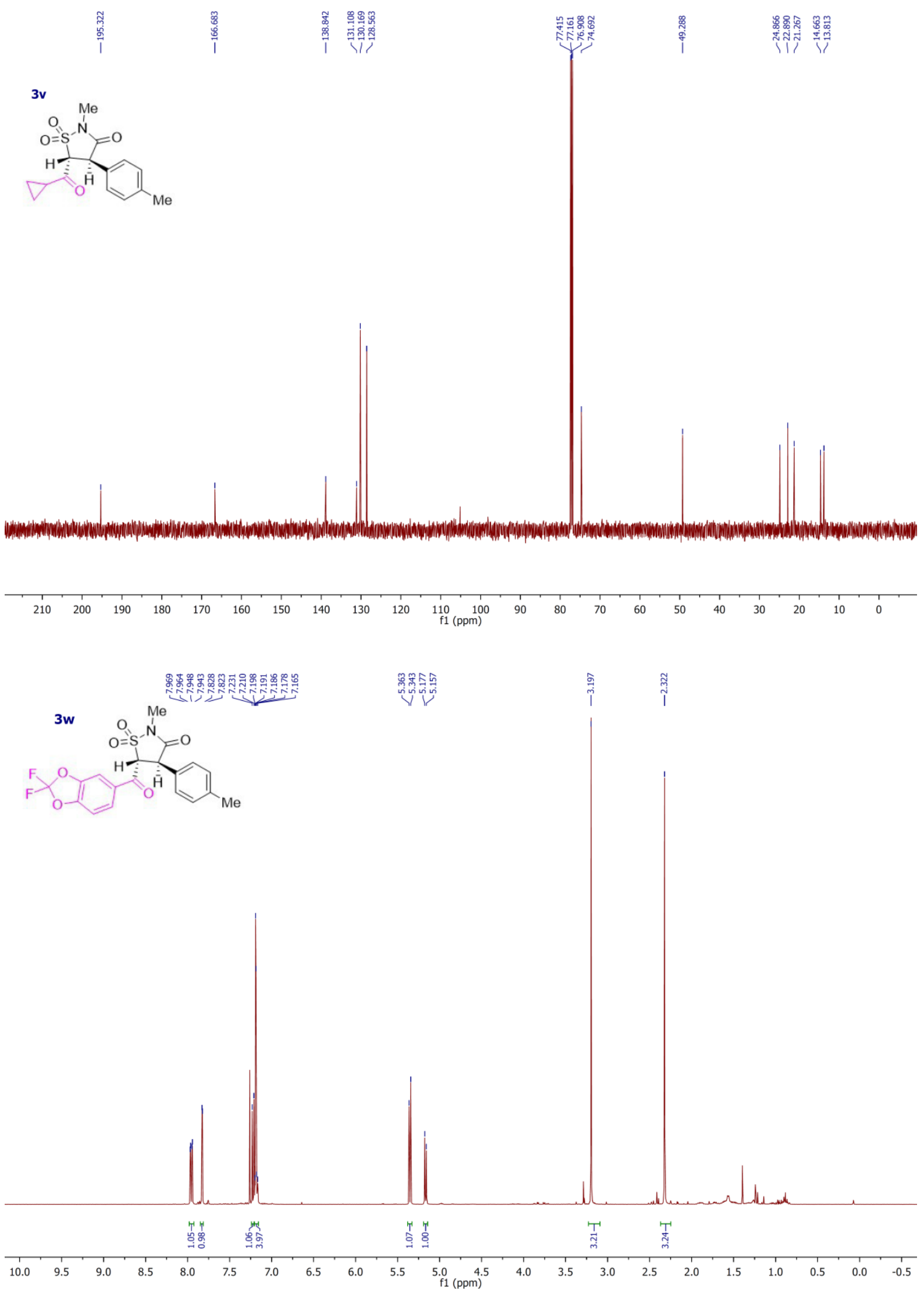

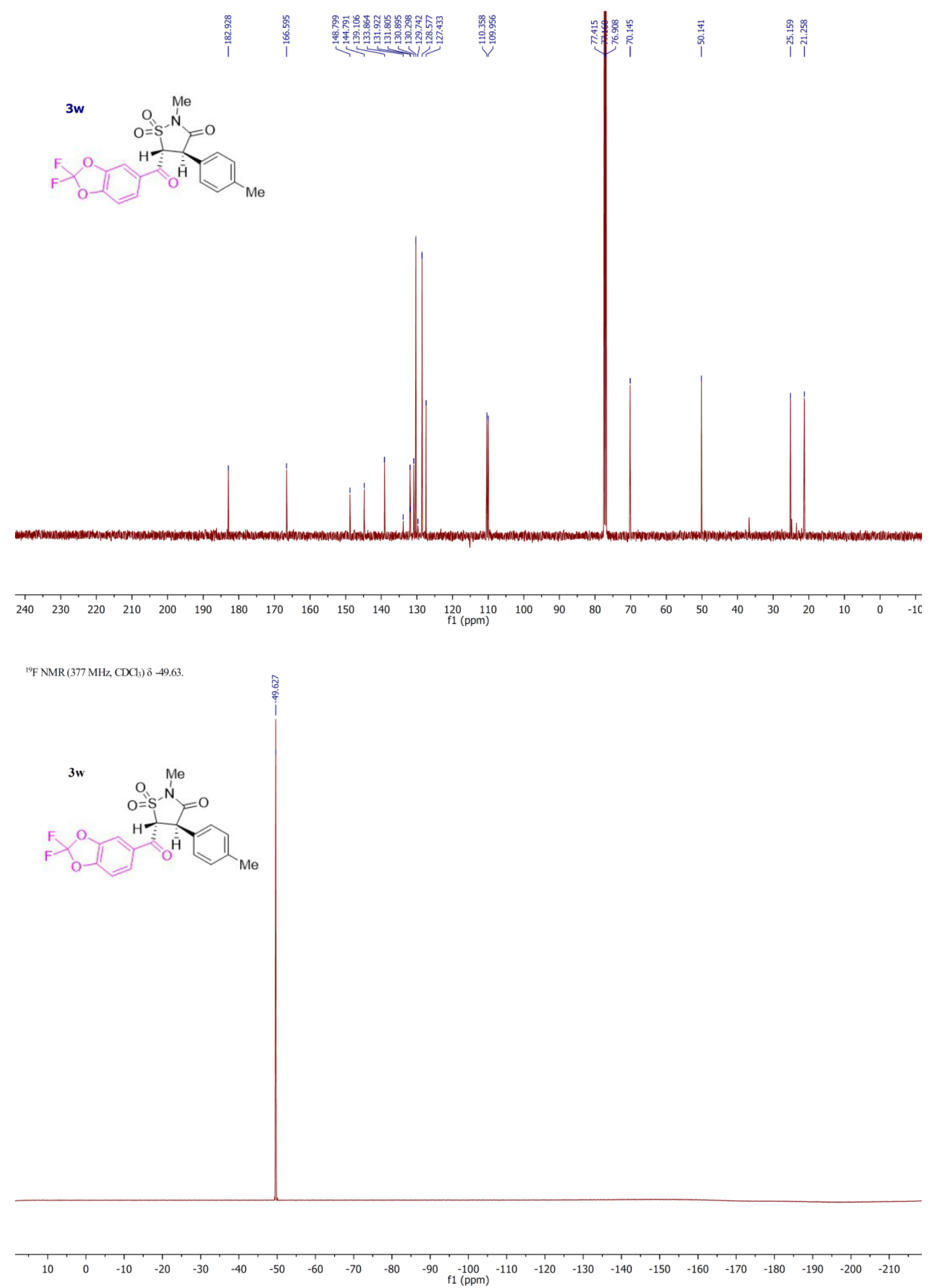

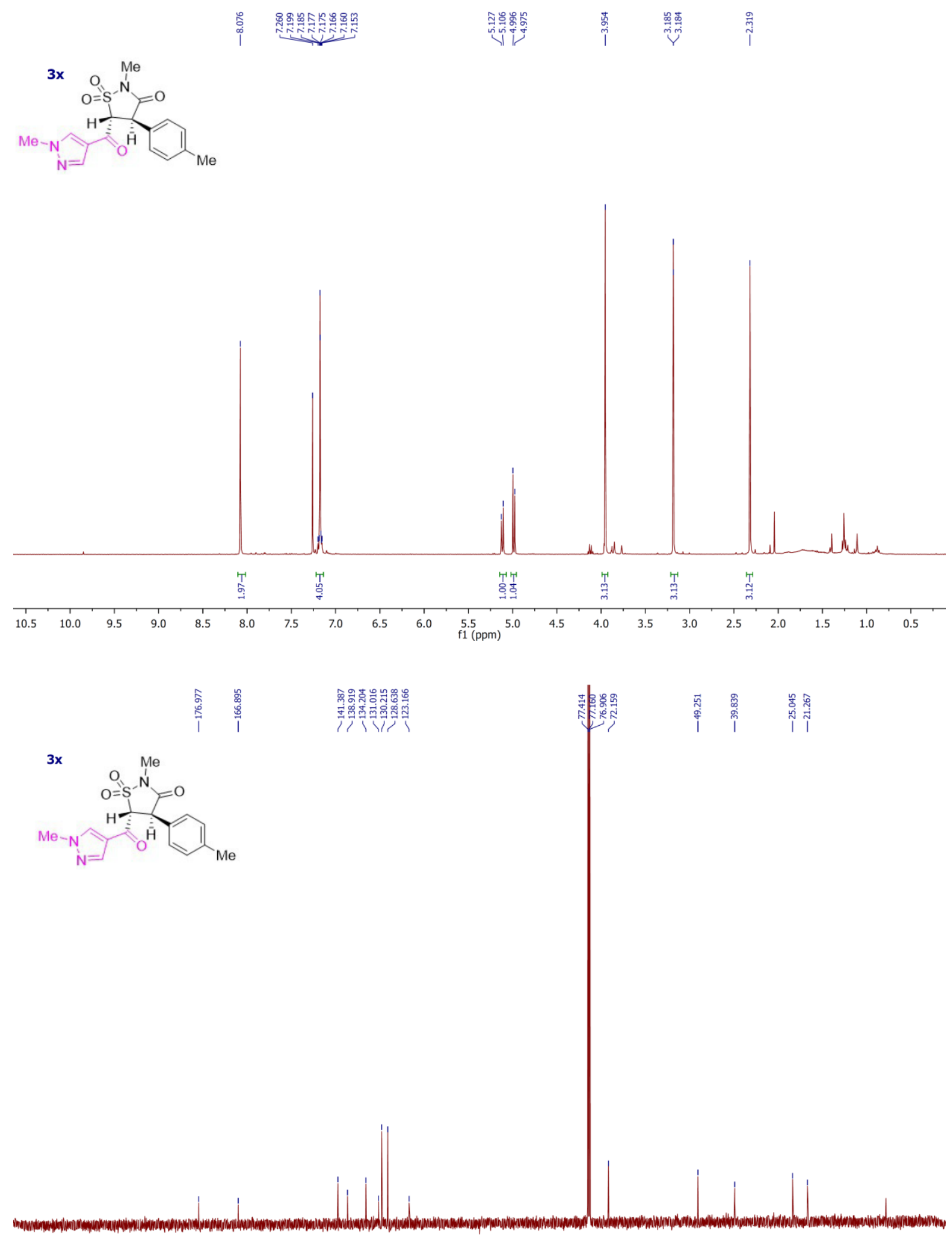

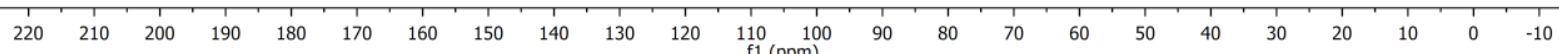



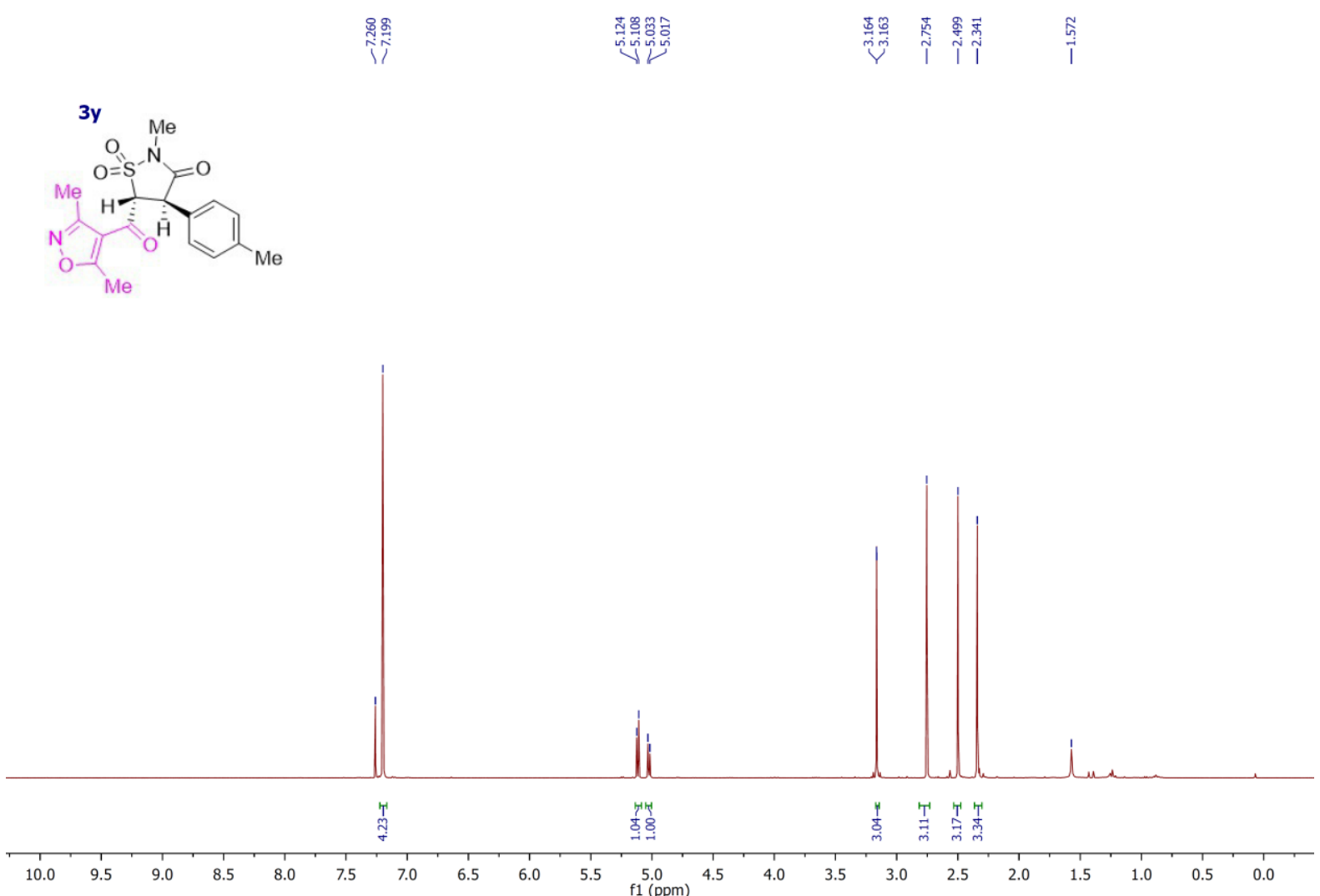

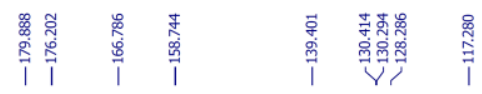
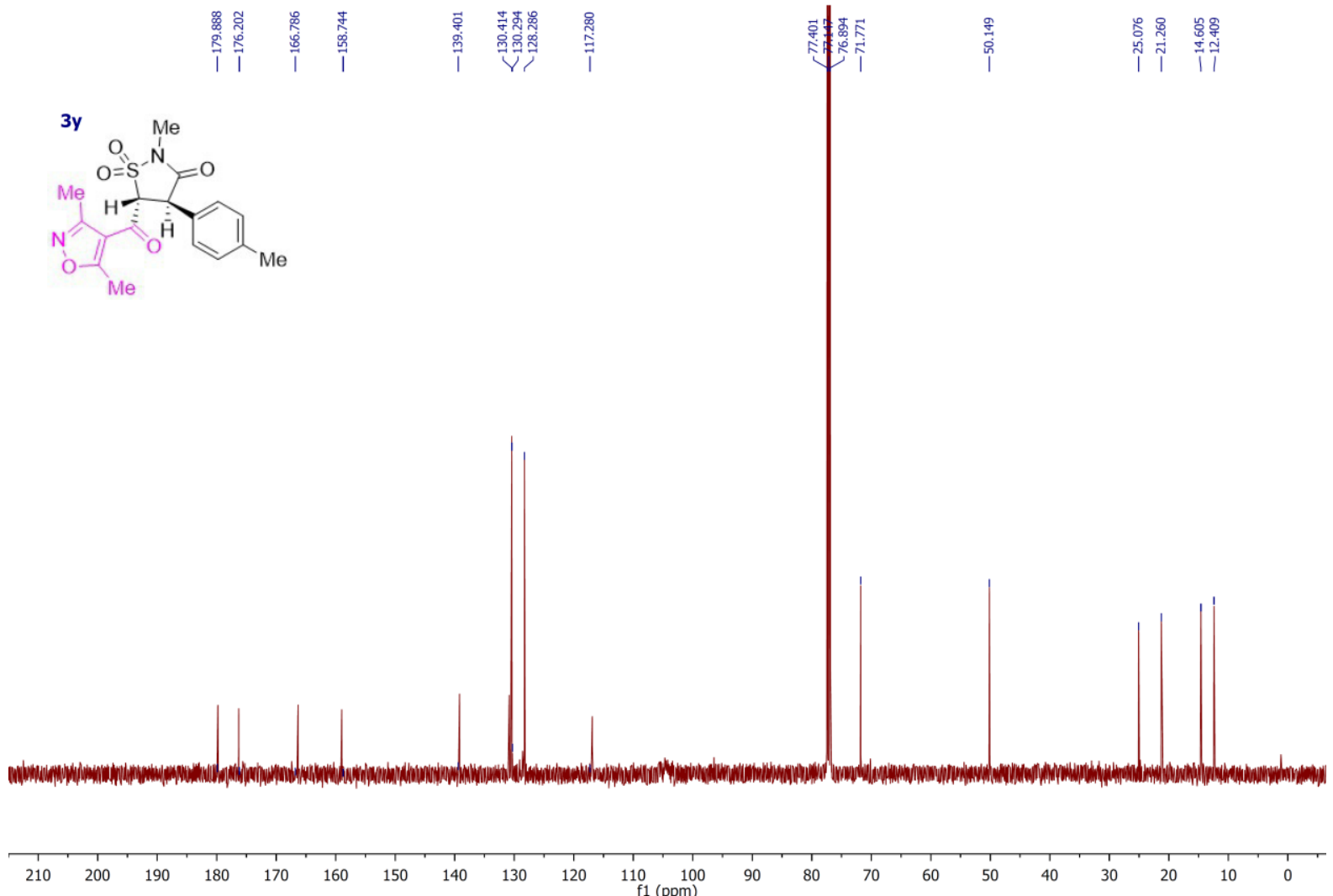
$3 z$

Ond

Me
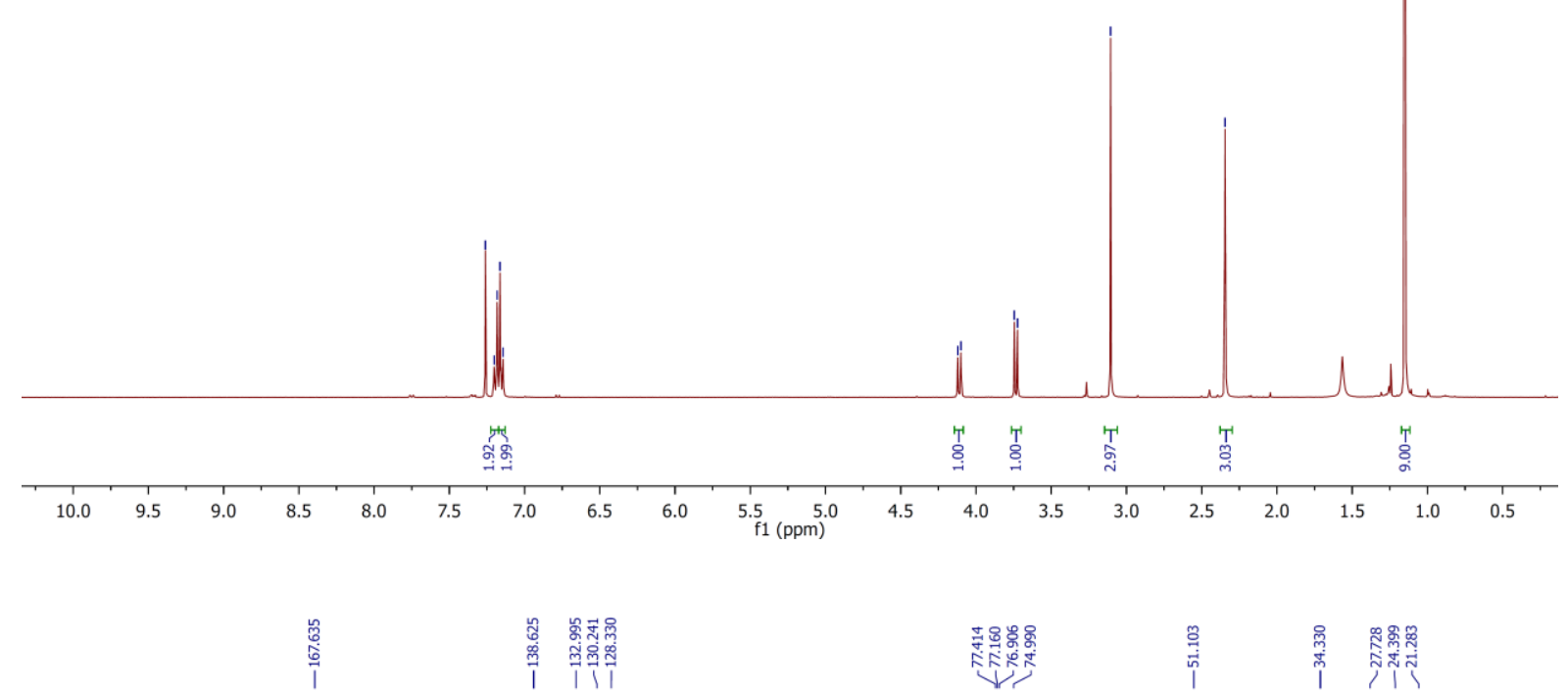

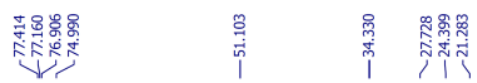

32
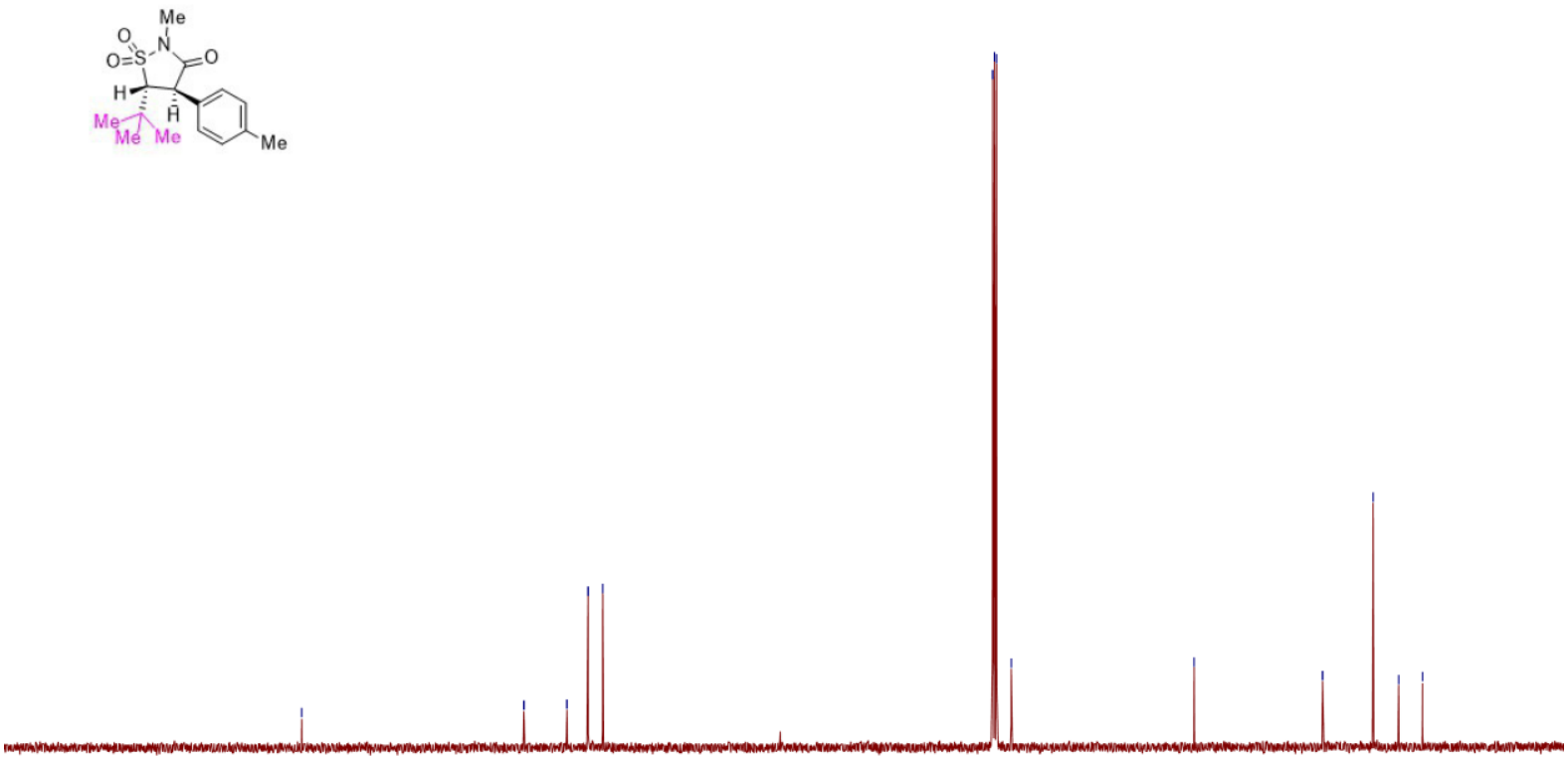

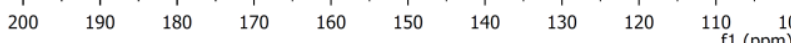



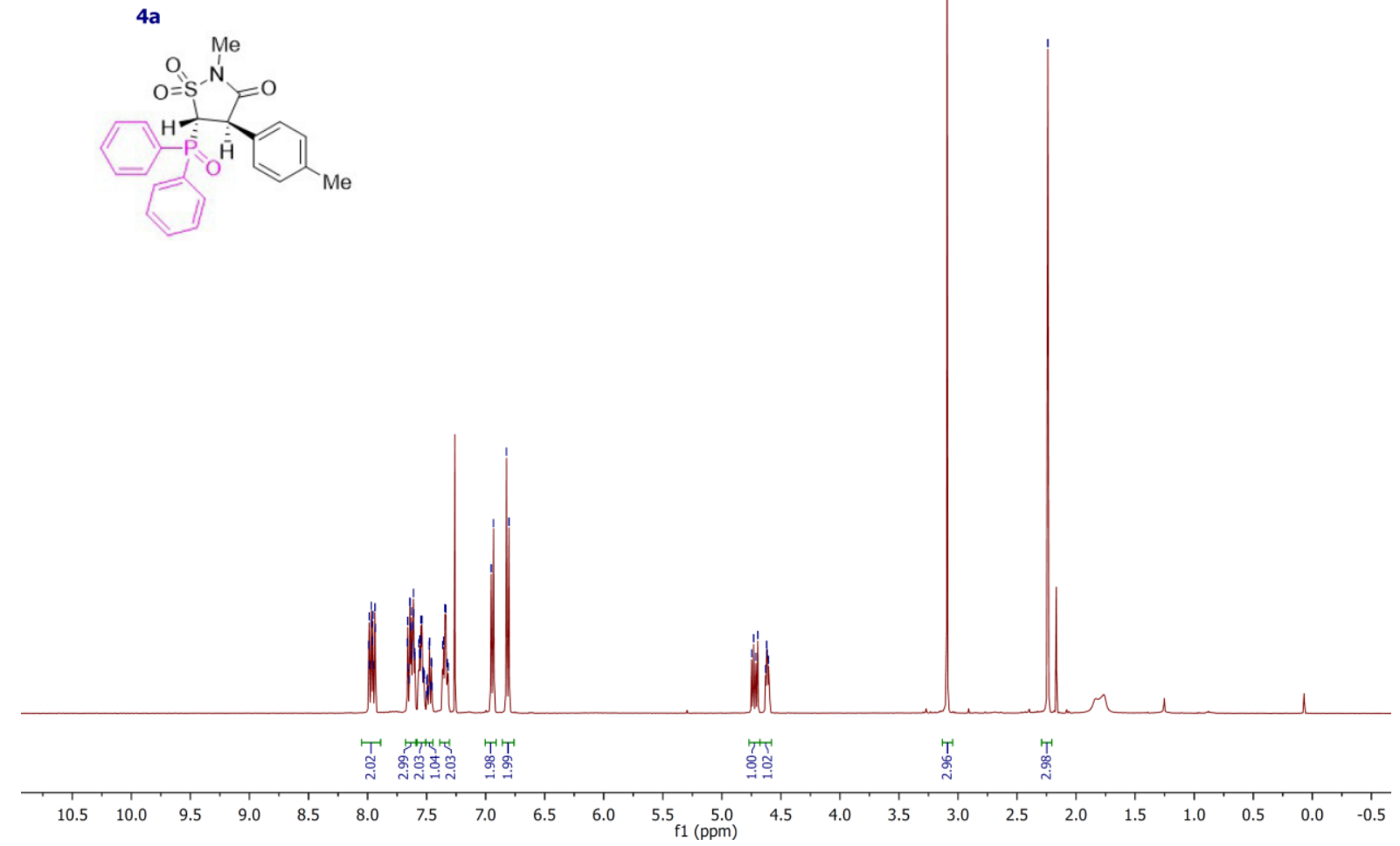

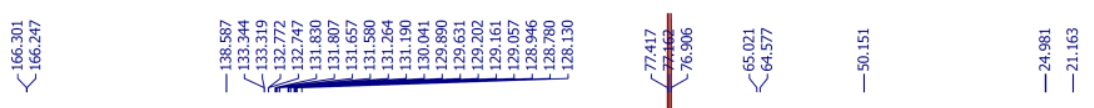

$4 a$
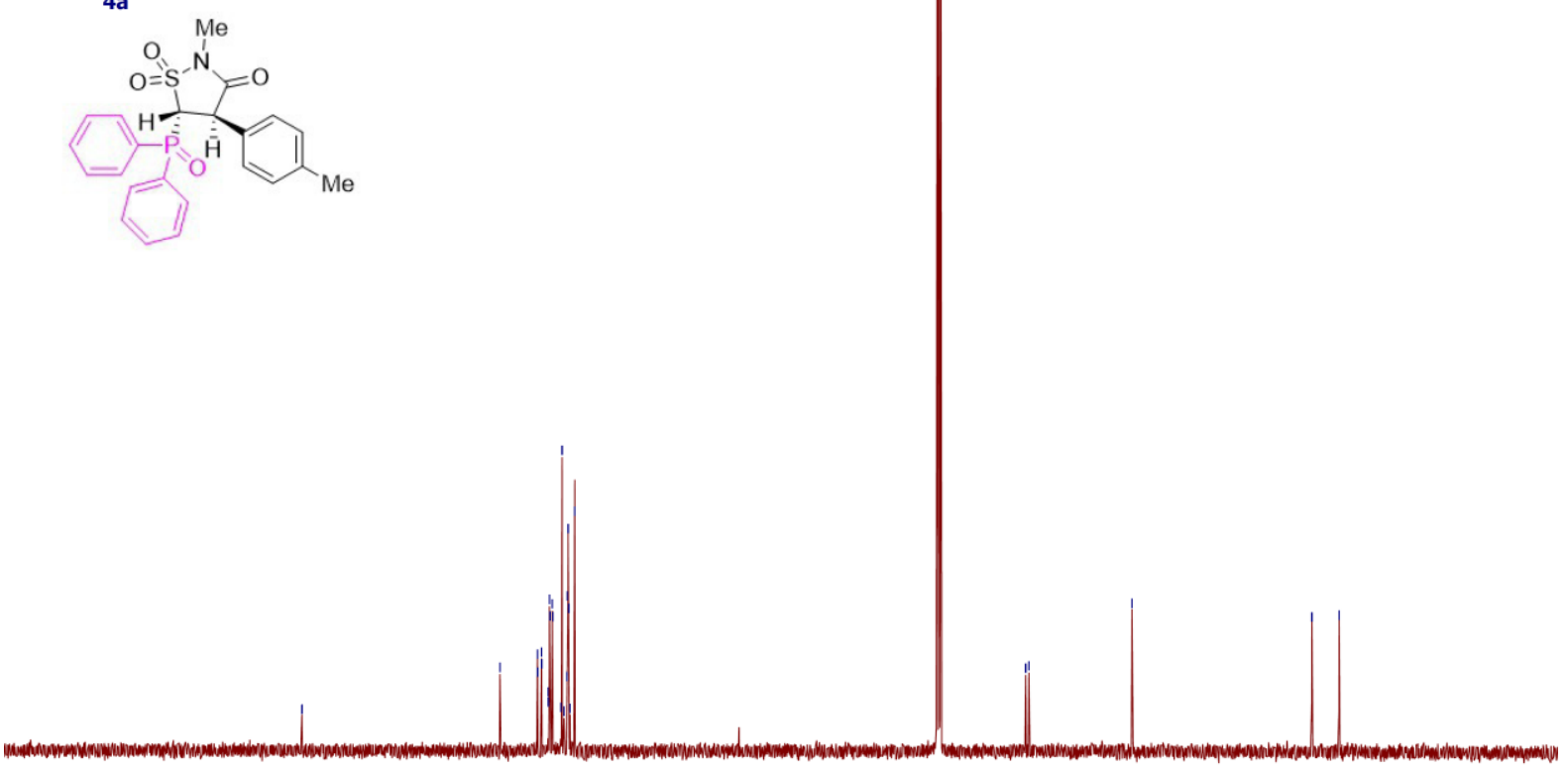

$\begin{array}{lllllllllll}200 & 190 & 180 & 170 & 160 & 150 & 140 & 130 & 120 & 110 & \begin{array}{c}100 \\ \mathrm{f} 1(\mathrm{ppm})\end{array}\end{array}$ 


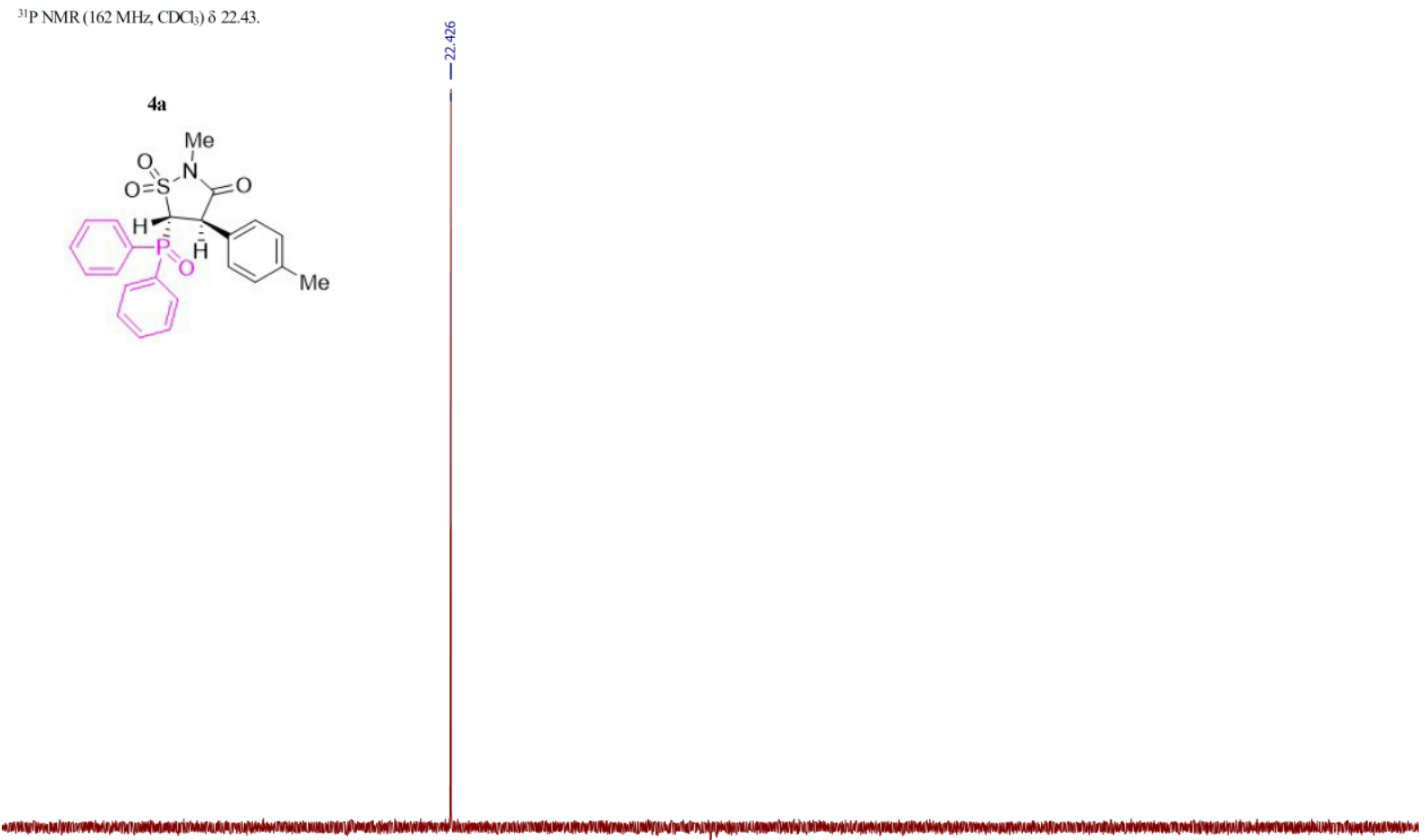

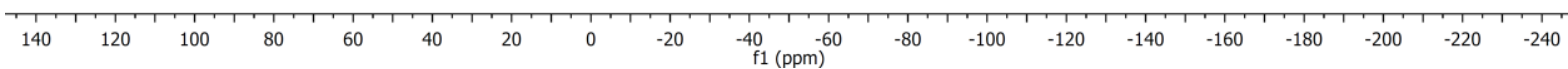

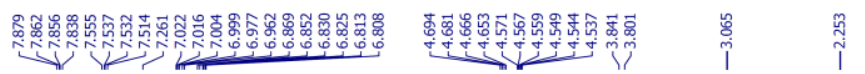

4b

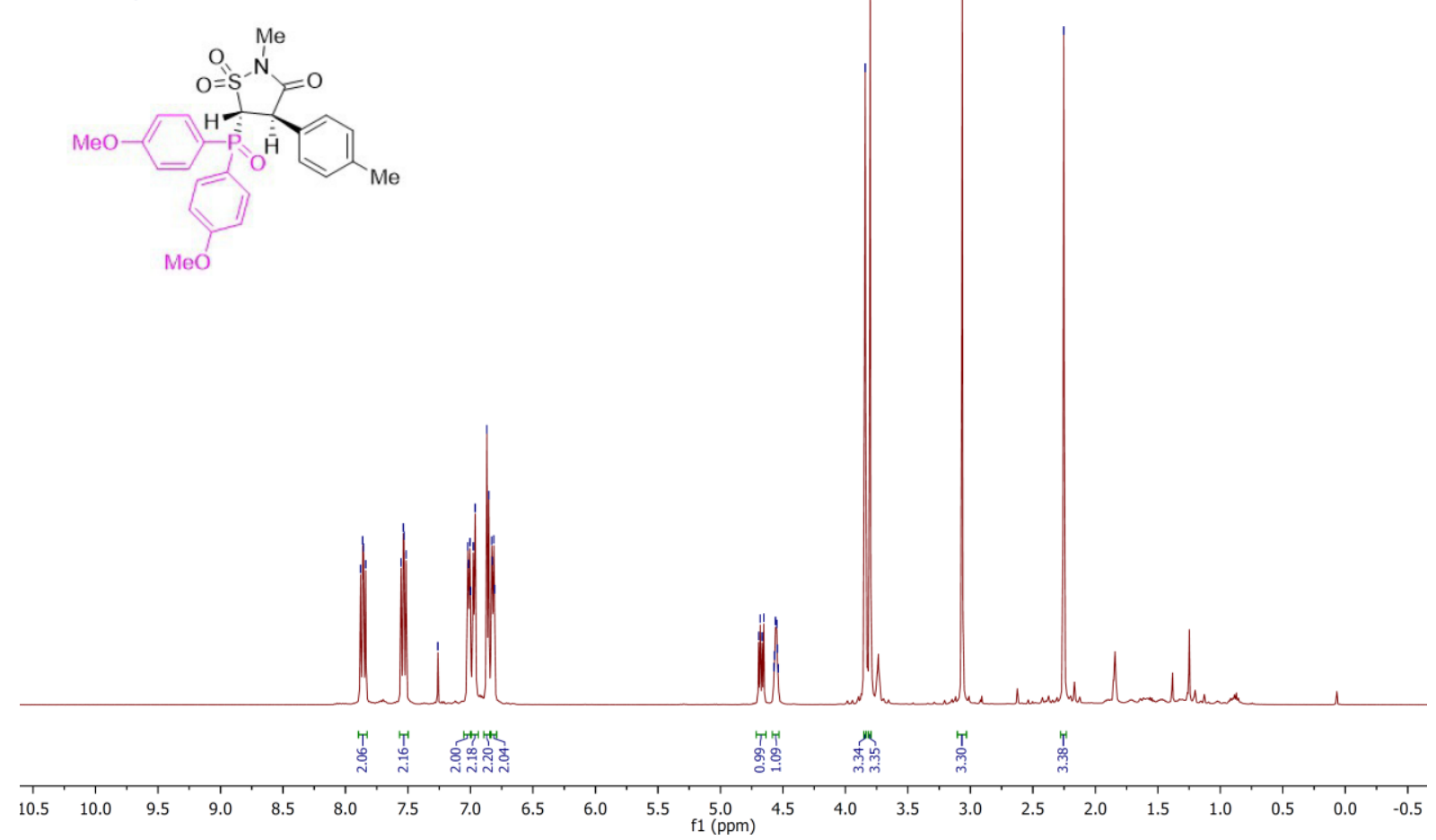



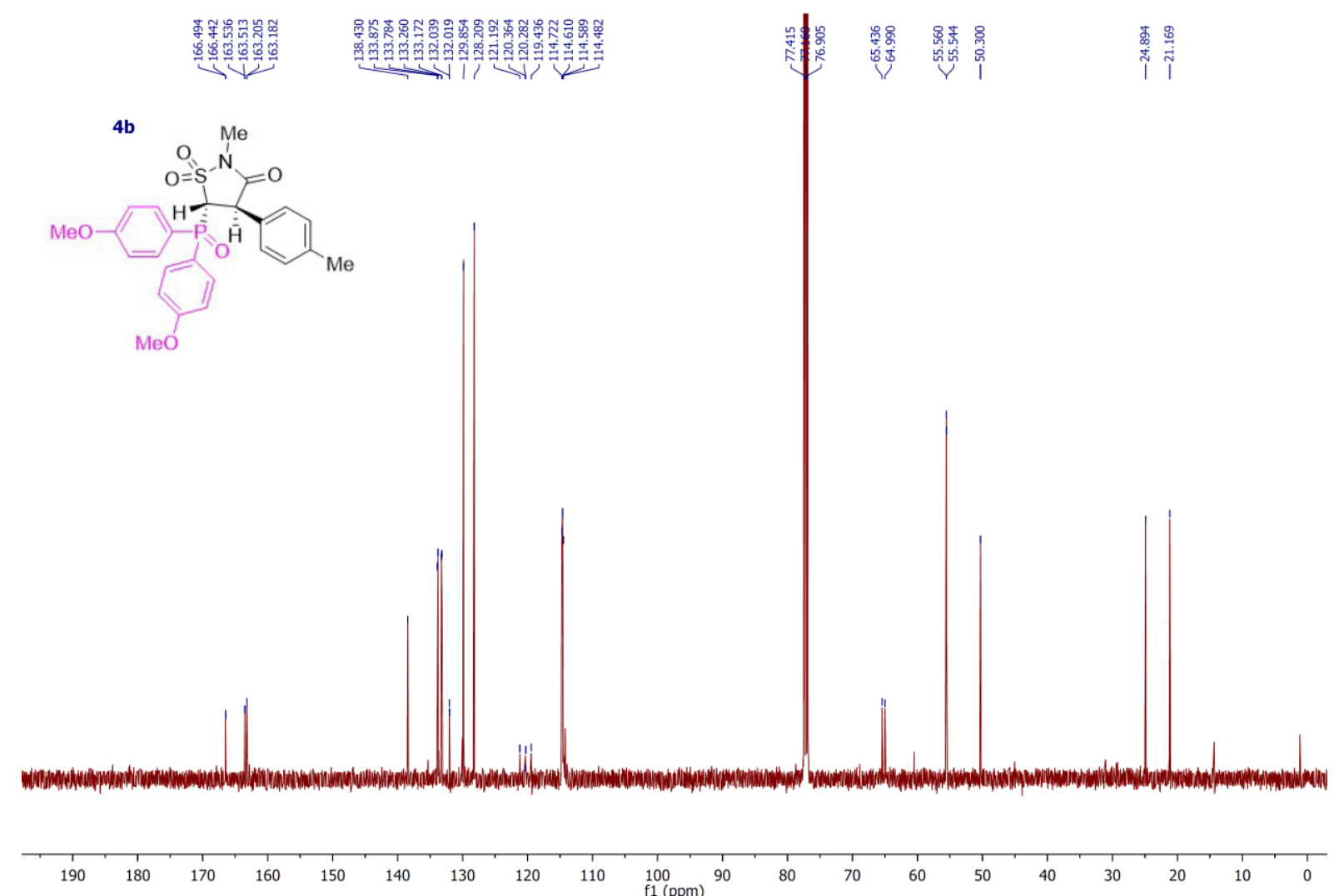

${ }^{31} \mathrm{P} \mathrm{NMR}\left(162 \mathrm{MHz}, \mathrm{CDCl}_{3}\right) \delta 22.96$.

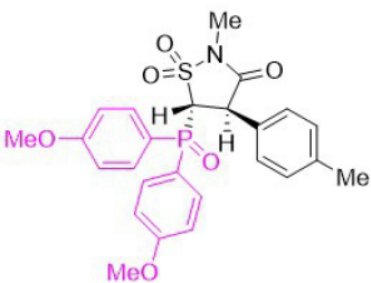

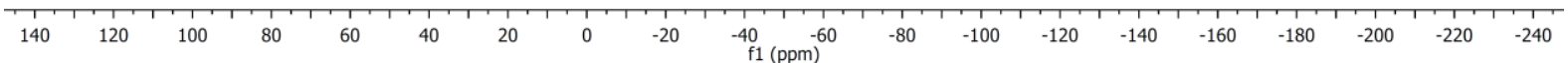



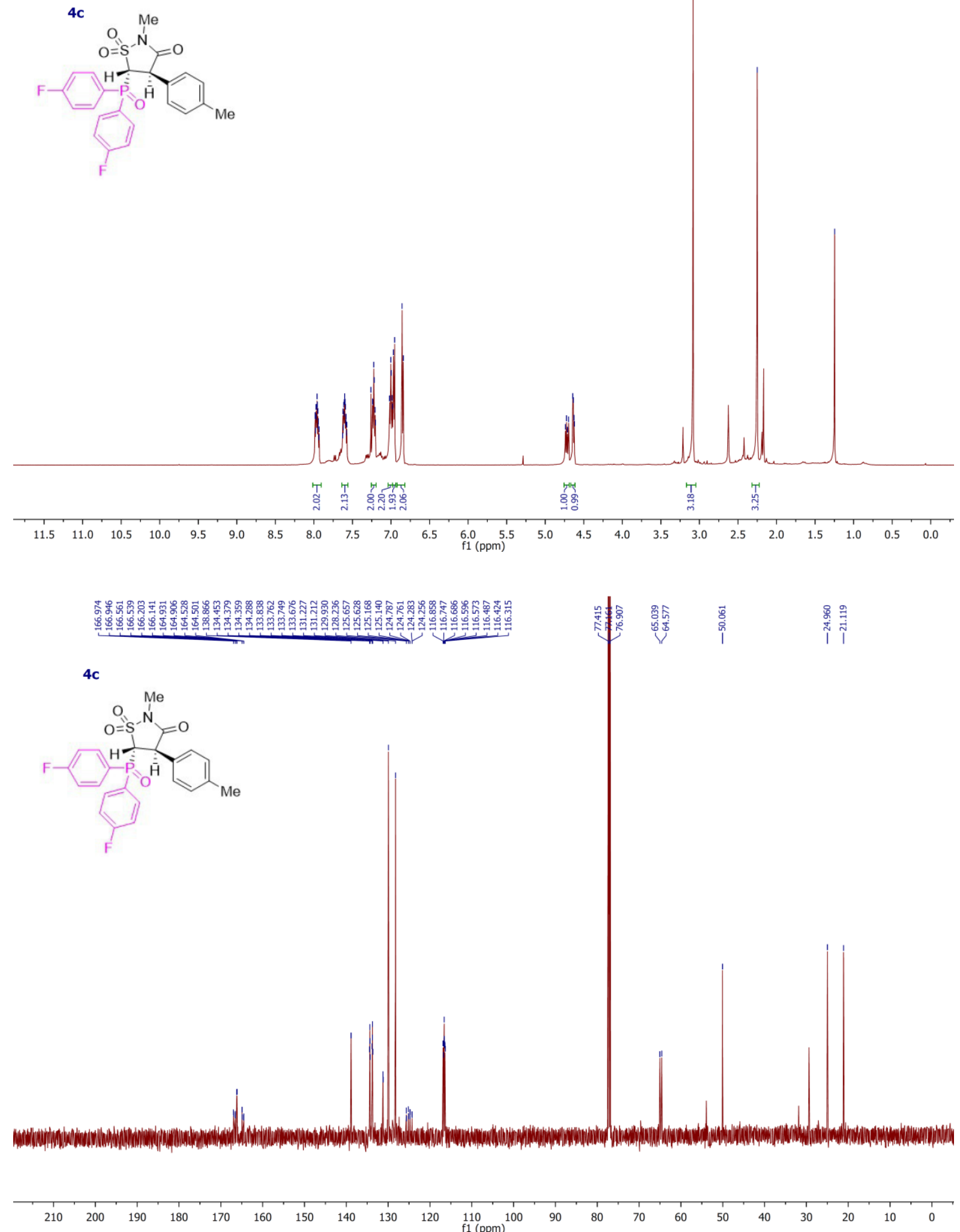
$4 c$

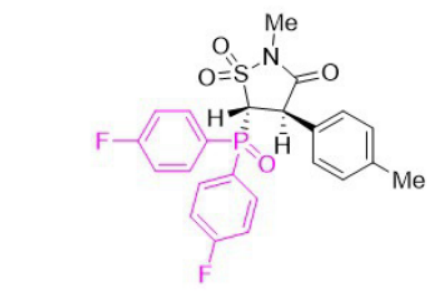

Me

\begin{tabular}{rlllllllllllllllllllllllllllll}
\hline & 1 & 0 & -10 & -20 & -30 & -40 & -50 & -60 & -70 & -80 & -90 & -100 & -110 & -120 & -130 & -140 & -150 & -160 & -170 & -180 & -190 & -200 & -210
\end{tabular}

${ }^{31} \mathrm{P} \mathrm{NMR}\left(202 \mathrm{MHz}, \mathrm{CDCl}_{3}\right) \delta 21.30$.

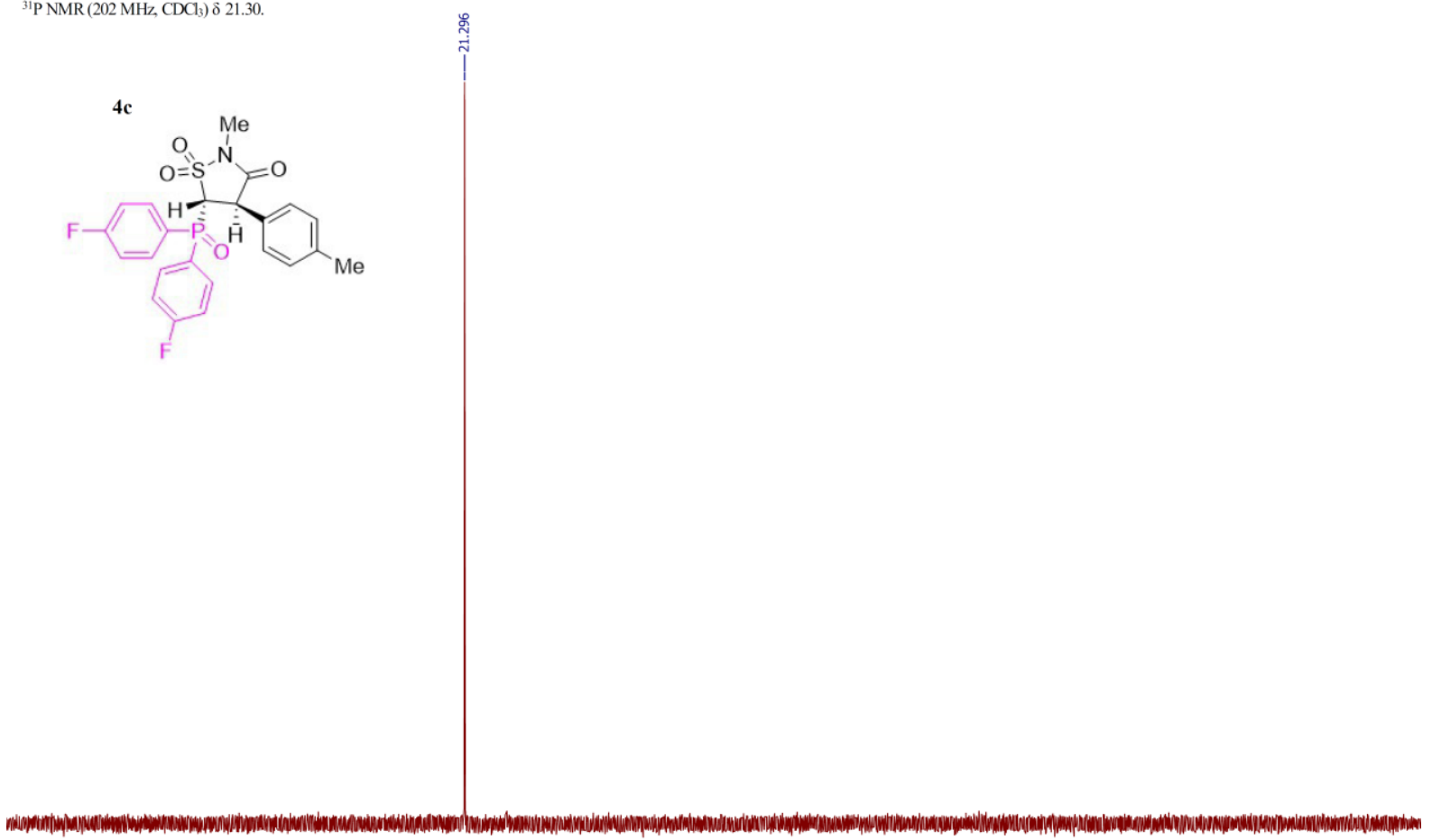

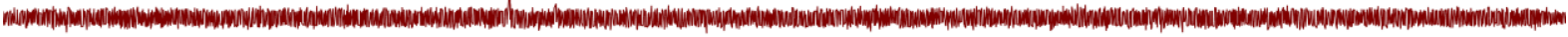

$\begin{array}{lllllllllllllllllllllllll}1 & 130 & 110 & 90 & 70 & 50 & 30 & 10 & -10 & -30 & -50 & -70 & -90 & -110 & -130 & -150 & -170 & -190 & -210 & -230 & -2\end{array}$



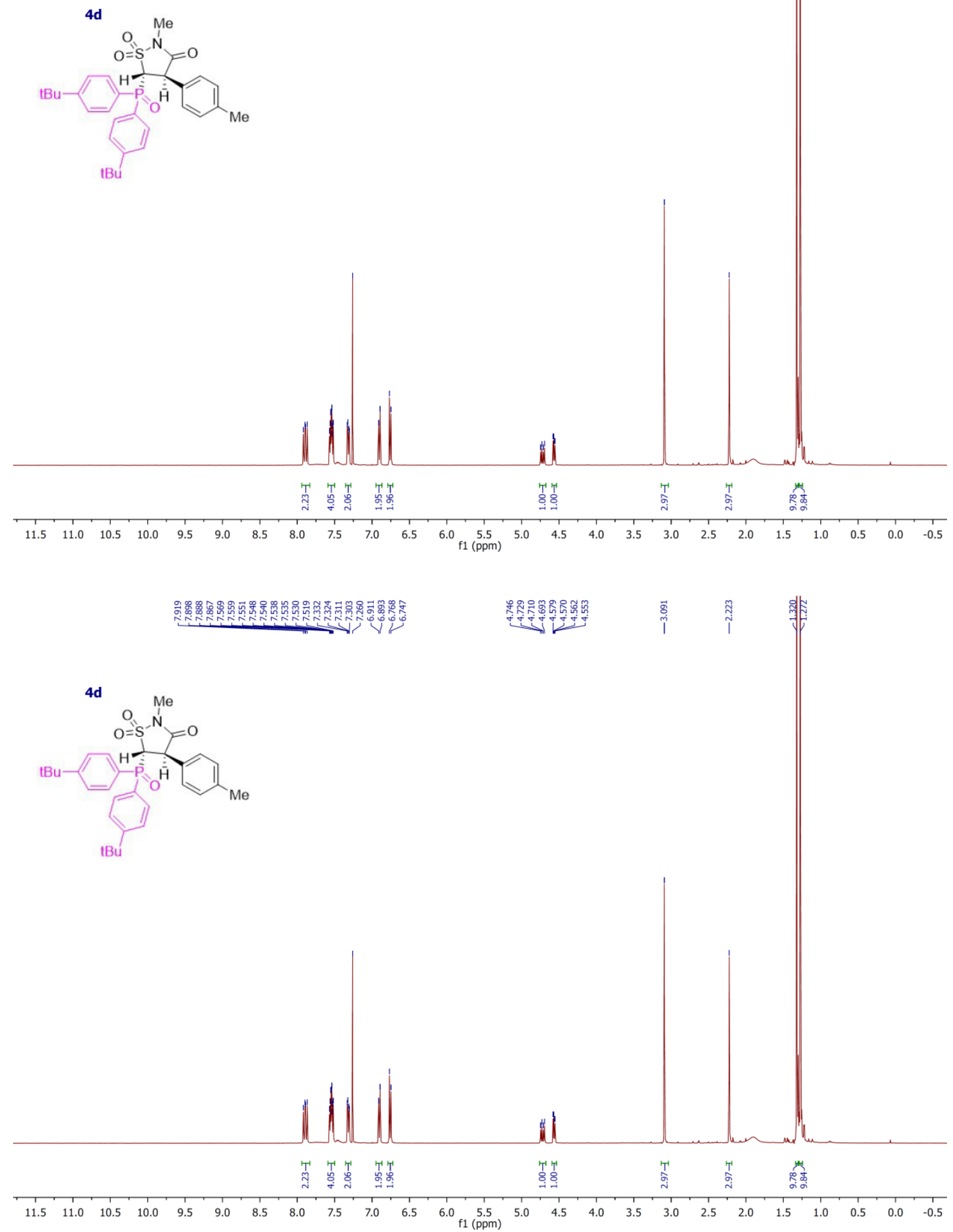


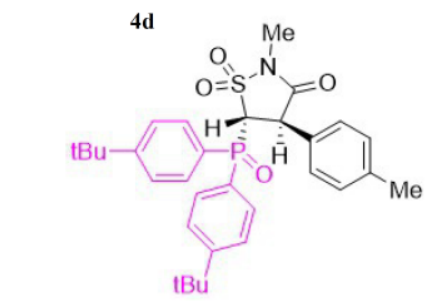

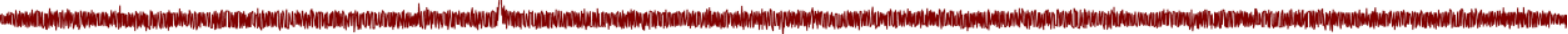

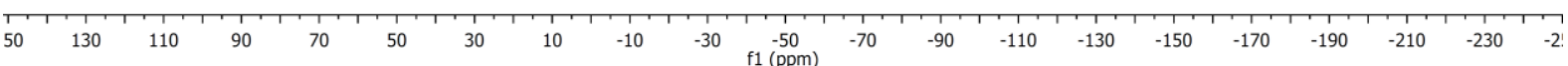

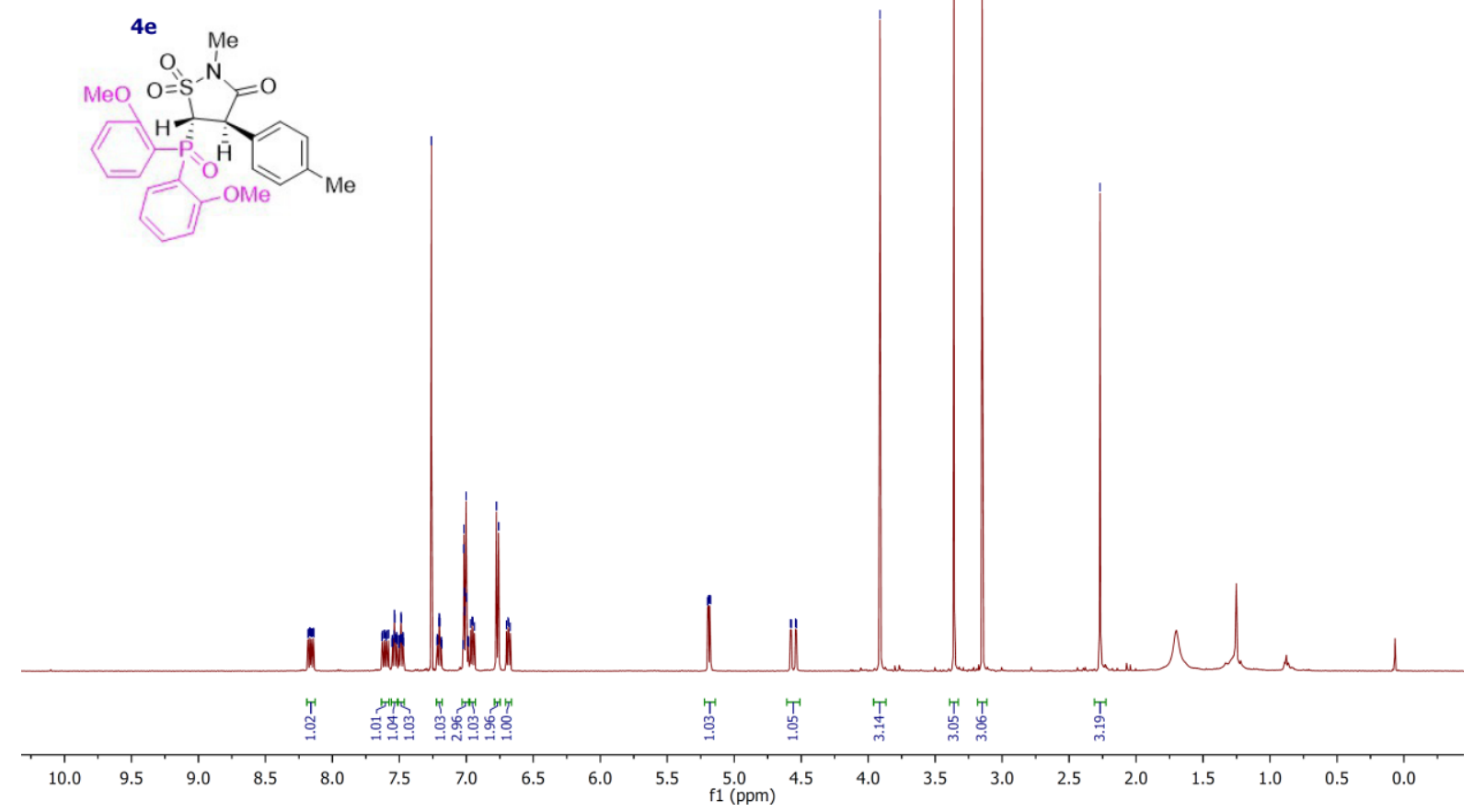



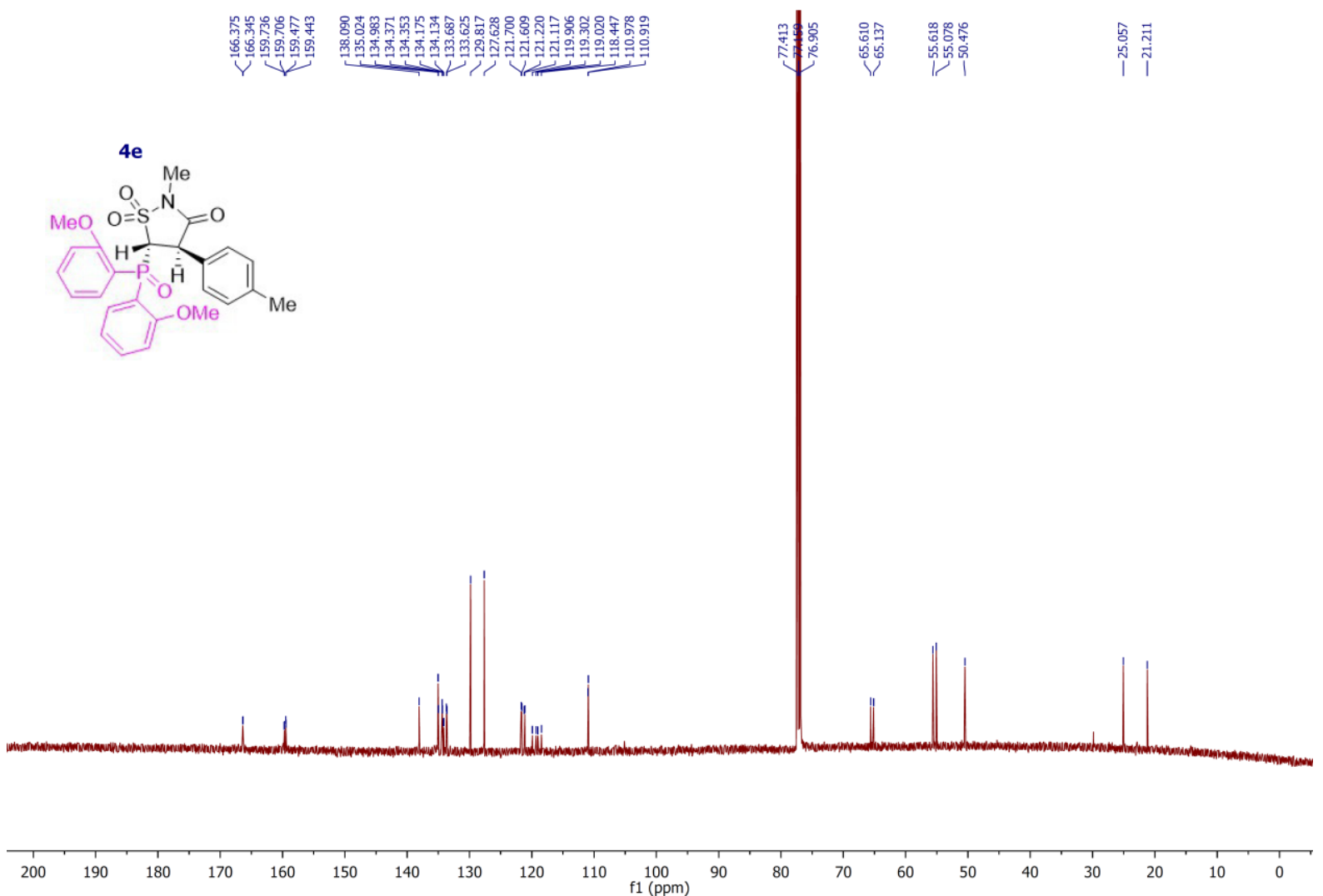

${ }^{31} \mathrm{P} \mathrm{NMR}\left(162 \mathrm{MHz}, \mathrm{CDCl}_{3}\right) \delta 23.34$.

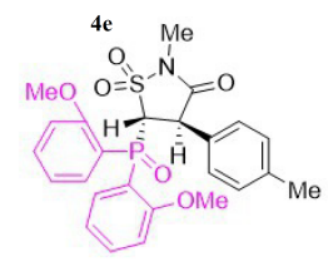

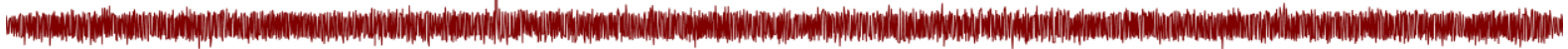

\begin{tabular}{lllllllllllllllllllll}
\hline 140 & 120 & 100 & 80 & 60 & 40 & 20 & 0 & -20 & -40 & -60 & -80 & -100 & -120 & -140 & -160 & -180 & -200 & -220 & -240
\end{tabular}




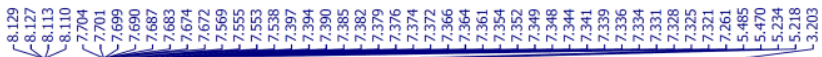
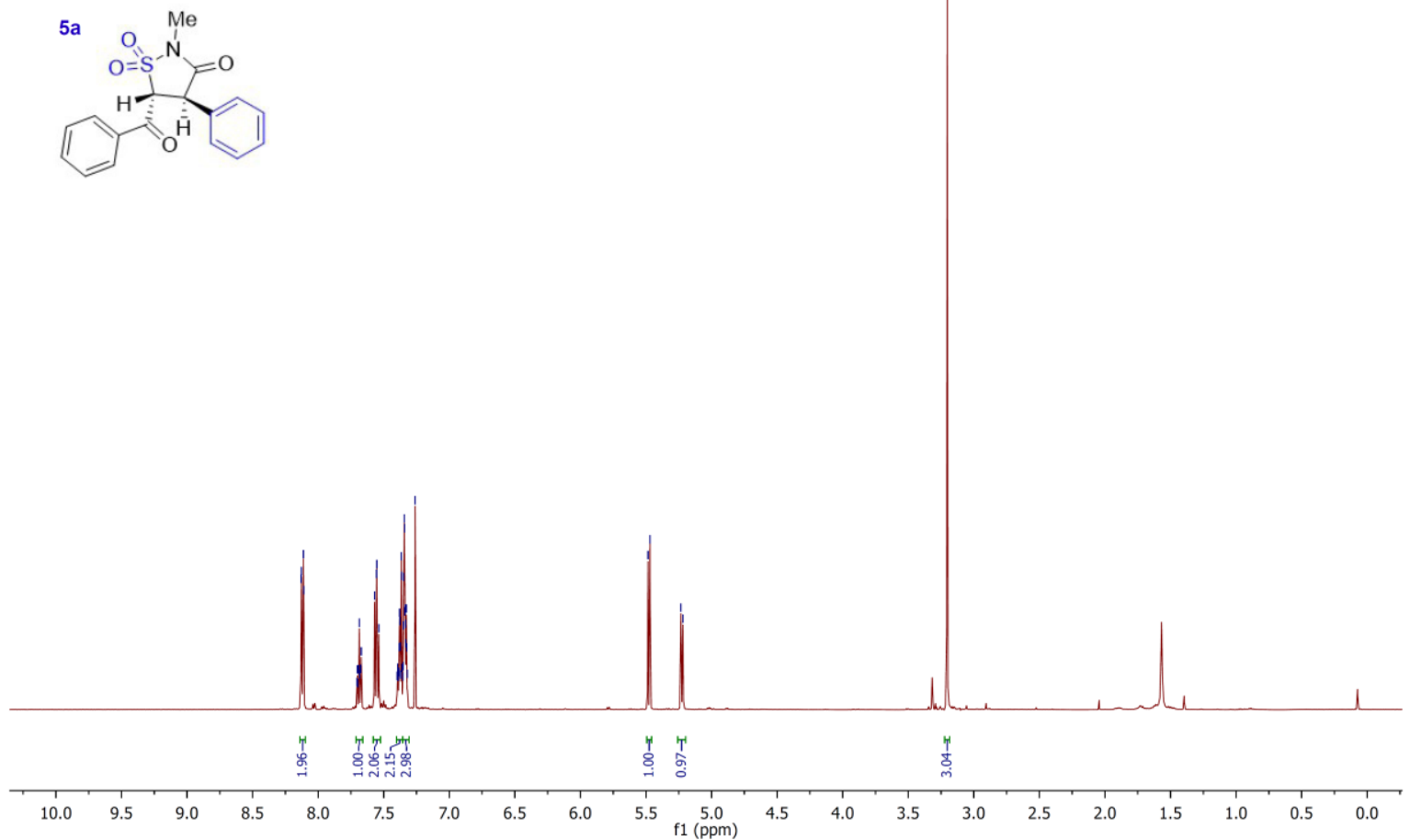

$5 a$

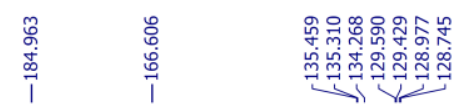

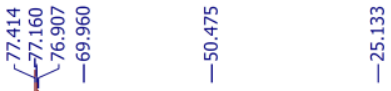
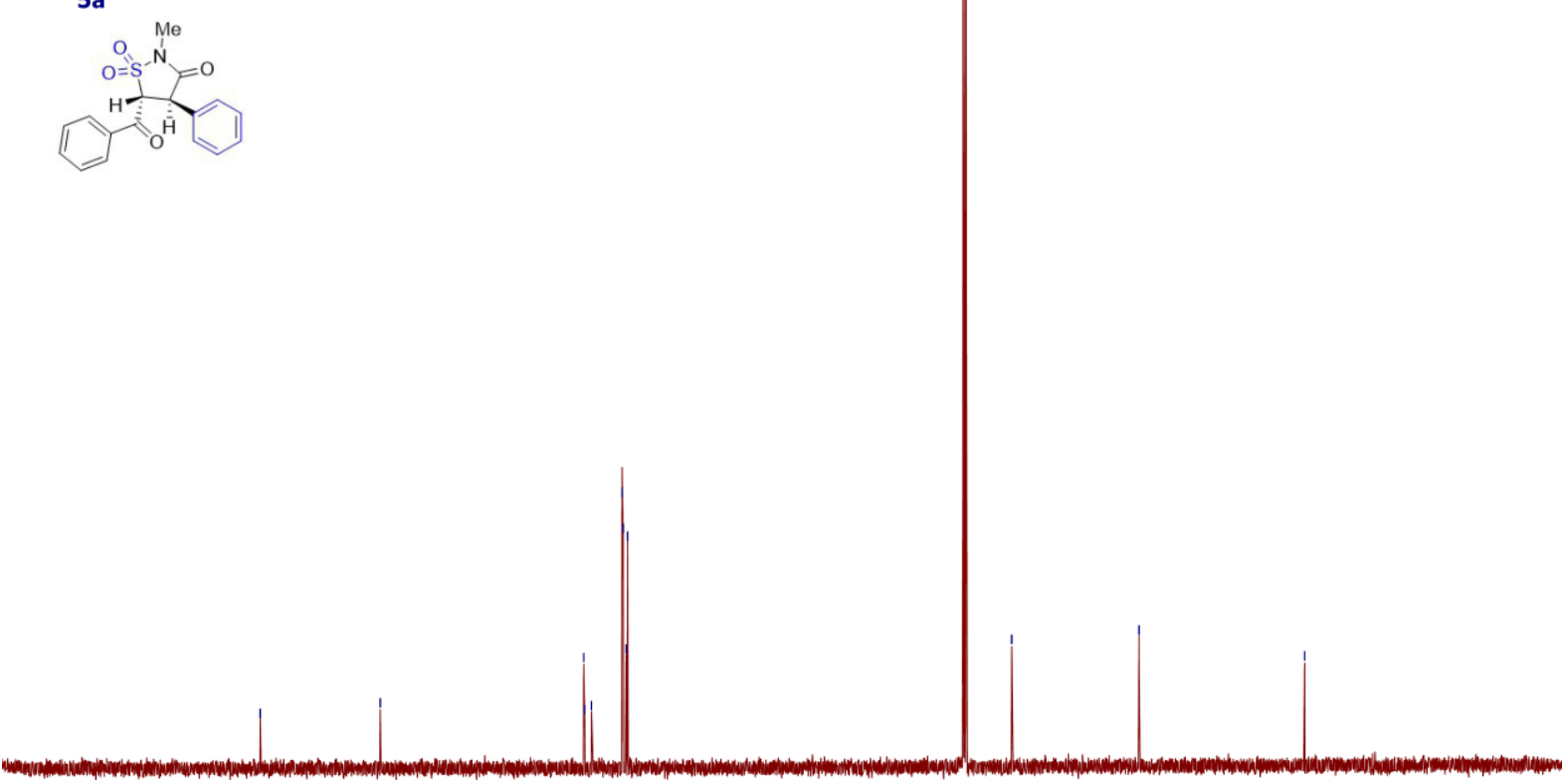

$\begin{array}{llllllllllllllllllllllll}220 & 210 & 200 & 190 & 180 & 170 & 160 & 150 & 140 & 130 & 120 & 110 & 100 & 90 & 80 & 70 & 60 & 50 & 40 & 30 & 20 & 10 & 0 & -10\end{array}$ 


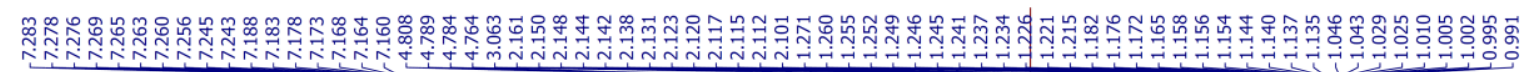

$5 b$

Sb Me

$\mathrm{O}=\mathrm{S}^{-\mathrm{N}}=0$

O
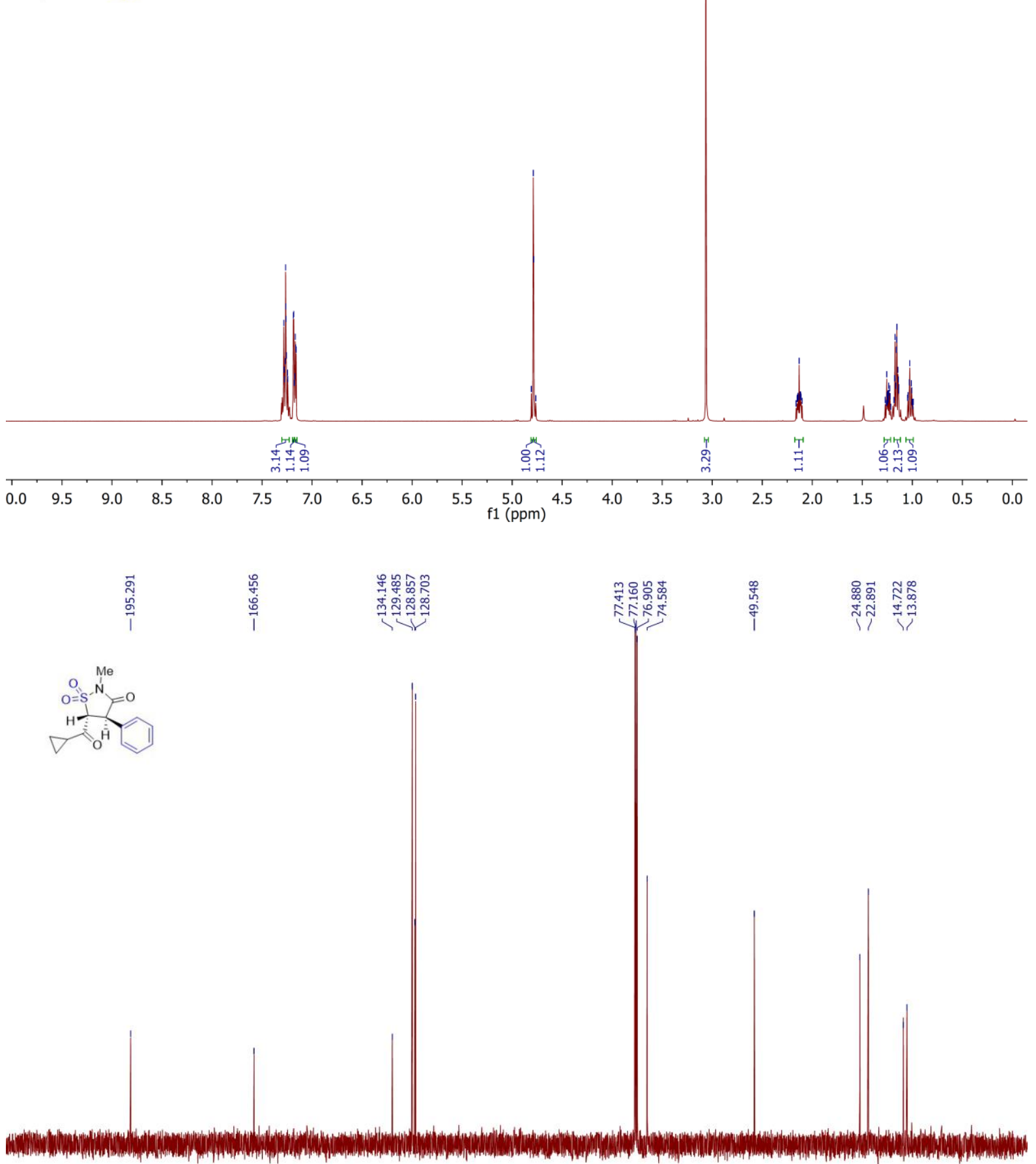

$\begin{array}{lllllllllllllllllllllllllllll}220 & 210 & 200 & 190 & 180 & 170 & 160 & 150 & 140 & 130 & 120 & \begin{array}{c}110 \\ \mathrm{f} 1(\mathrm{ppm})\end{array} & 100 & 80 & 70 & 60 & 50 & 40 & 30 & 20 & 10 & 0 & -10\end{array}$ 


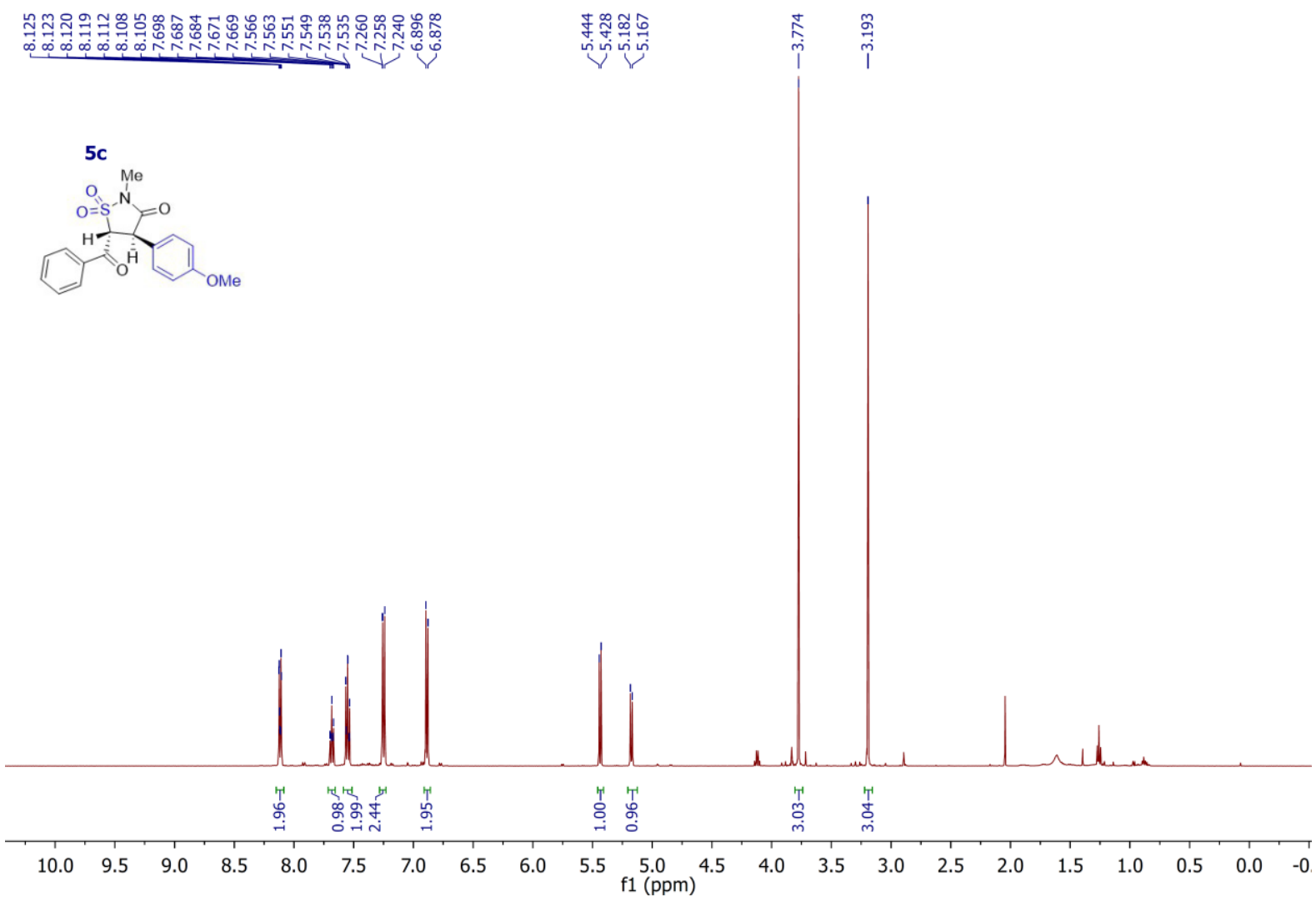

$5 \mathbf{c}$
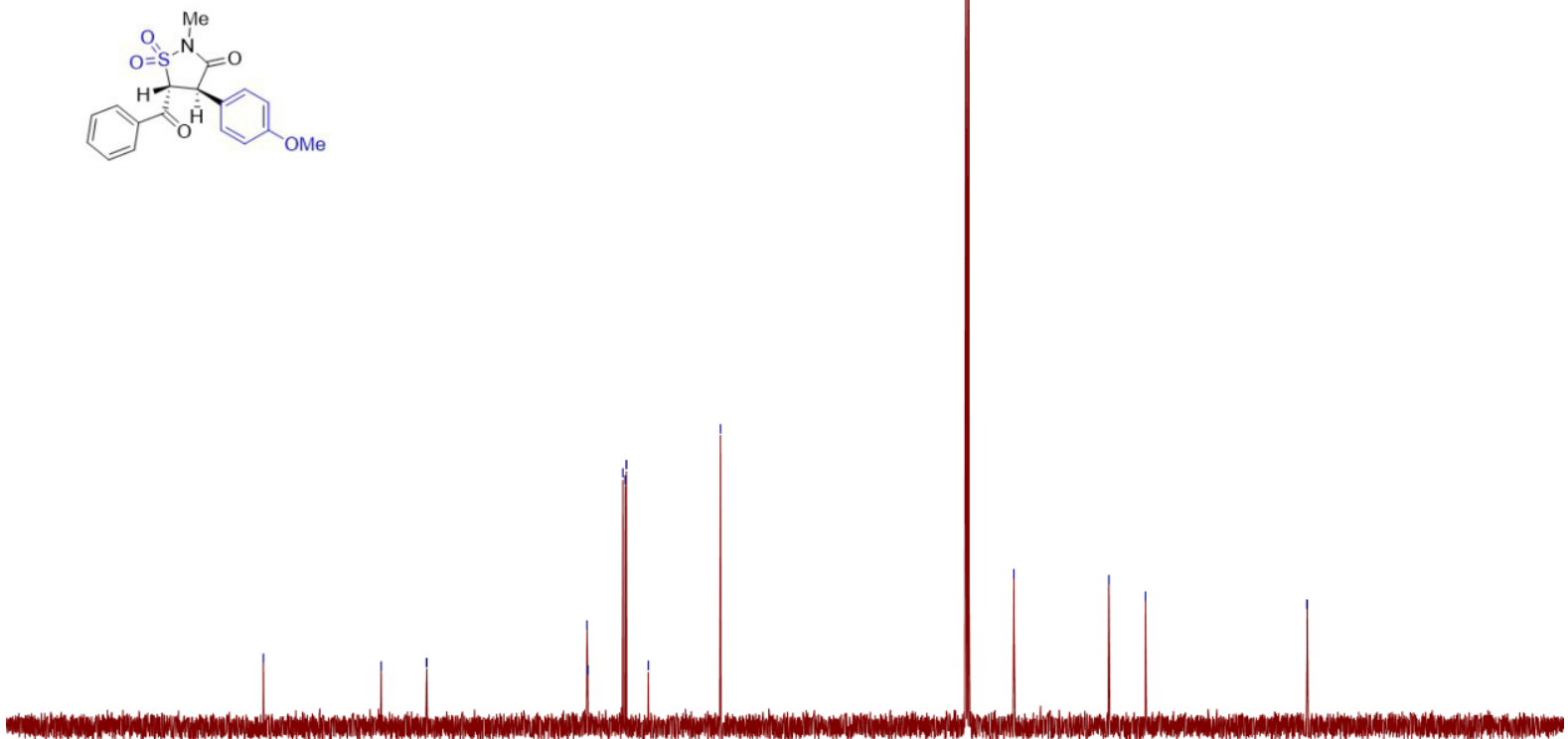

$\begin{array}{lllllllllllllllllllllllll}220 & 210 & 200 & 190 & 180 & 170 & 160 & 150 & 140 & 130 & 120 & 110 & 100 & 90 & 80 & 70 & 60 & 50 & 40 & 30 & 20 & 10 & 0 & -10\end{array}$ 

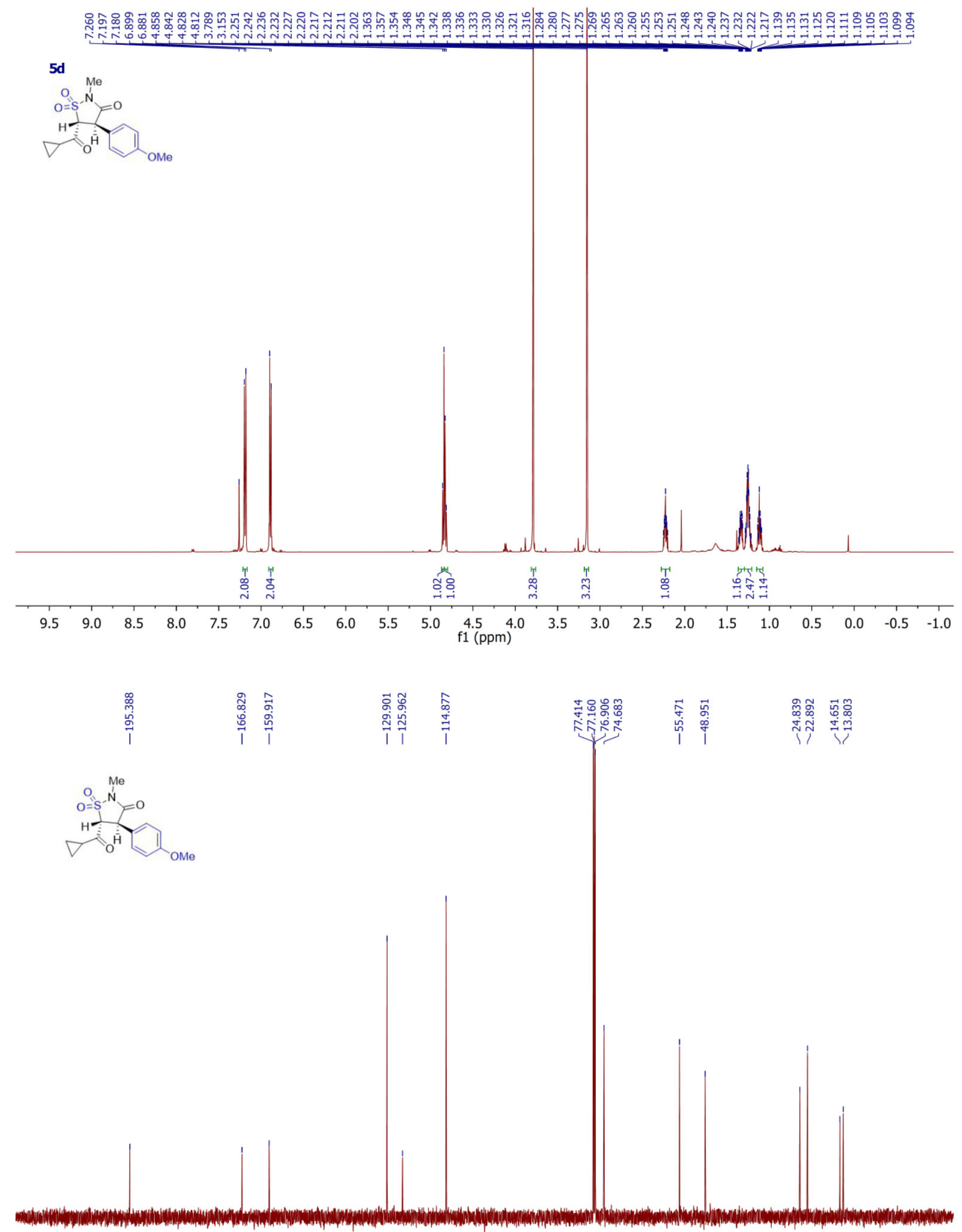

\begin{tabular}{llllllllllllllllllllllllllllll}
\hline 220 & 210 & 200 & 190 & 180 & 170 & 160 & 150 & 140 & 130 & 120 & $\begin{array}{c}110 \\
\mathrm{f} 1(\mathrm{ppm})\end{array}$ & 100 & 90 & 70 & 60 & 50 & 40 & 30 & 20 & 10 & 0 & -10
\end{tabular} 


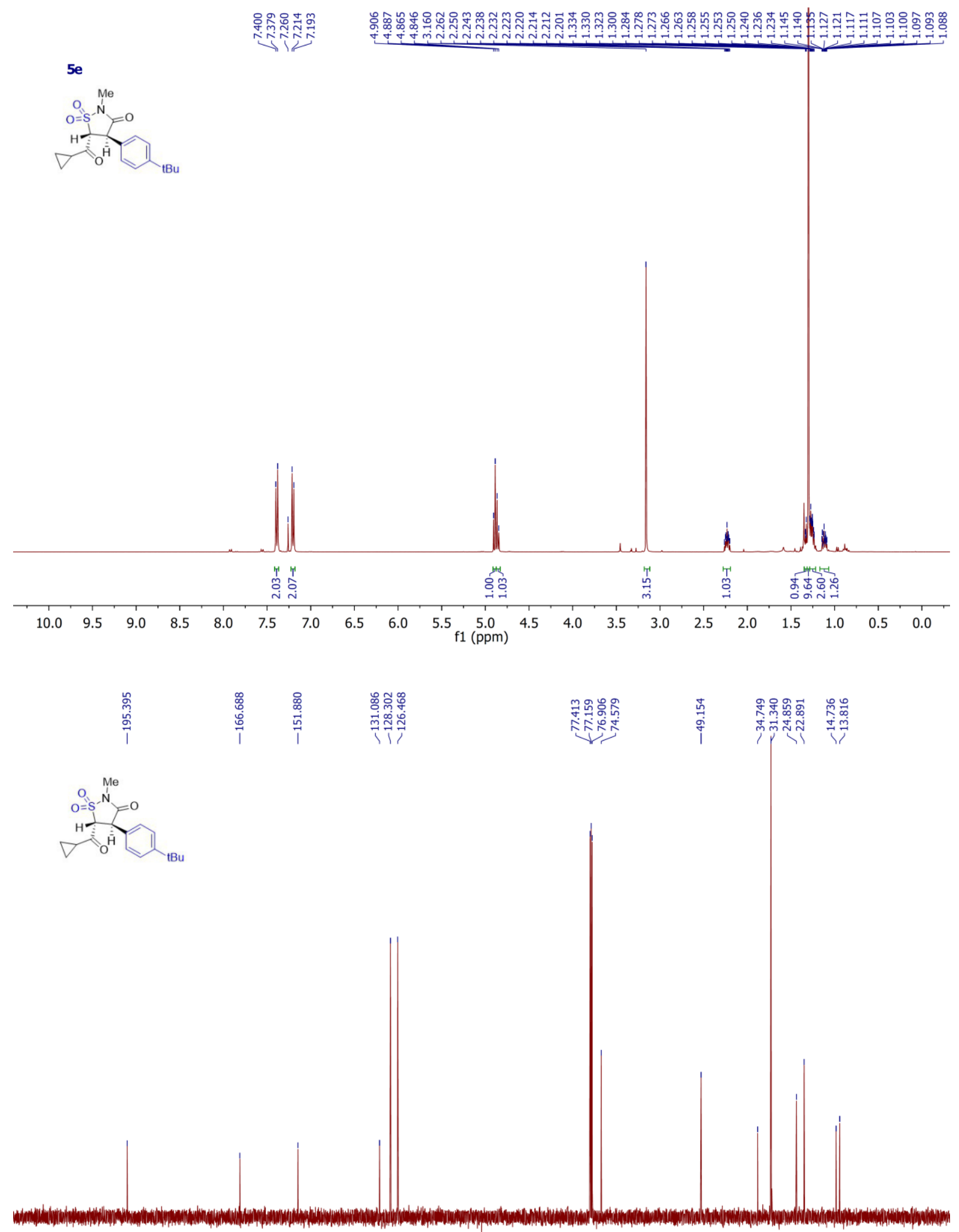

$\begin{array}{lllllllllllllllllllllllllll}220 & 210 & 200 & 190 & 180 & 170 & 160 & 150 & 140 & 130 & 120 & \begin{array}{c}110 \\ \mathrm{f}(\mathrm{ppm})\end{array} & 100 & 80 & 70 & 60 & 50 & 40 & 30 & 20 & 10 & 0 & -10\end{array}$ 

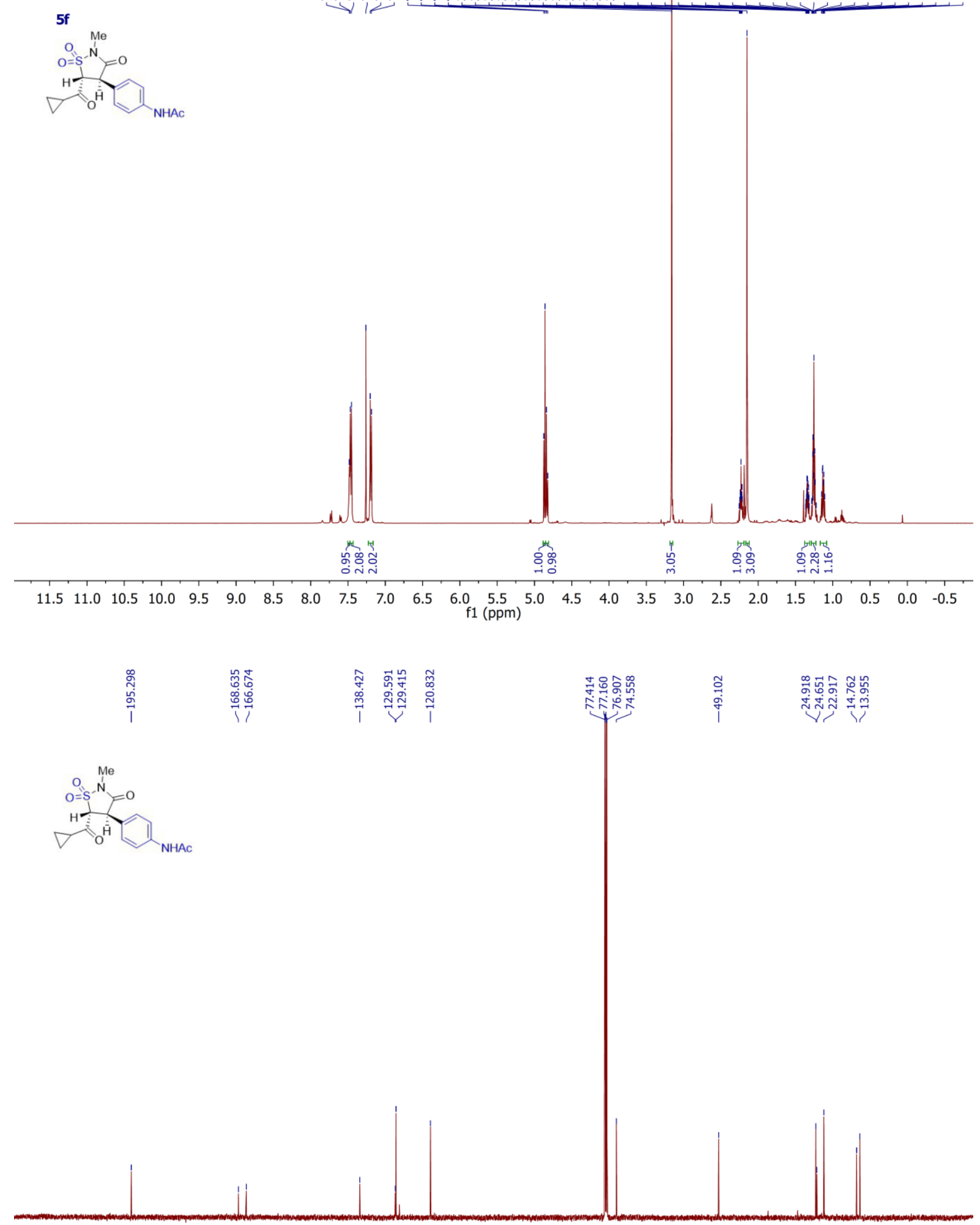

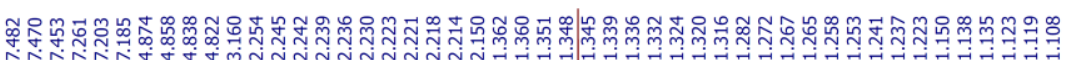

(ppm)

$\begin{array}{rrrrrrrrrrrrrrrrrrrrrrrrrr}1 & 220 & 210 & 200 & 190 & 180 & 170 & 160 & 150 & 140 & 130 & 120 & \begin{array}{c}110 \\ \mathrm{f} 1(\mathrm{ppm})\end{array} & 100 & 80 & 70 & 60 & 50 & 40 & 30 & 20 & 10 & 0 & -10\end{array}$ 


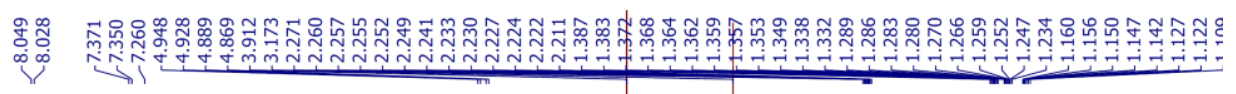
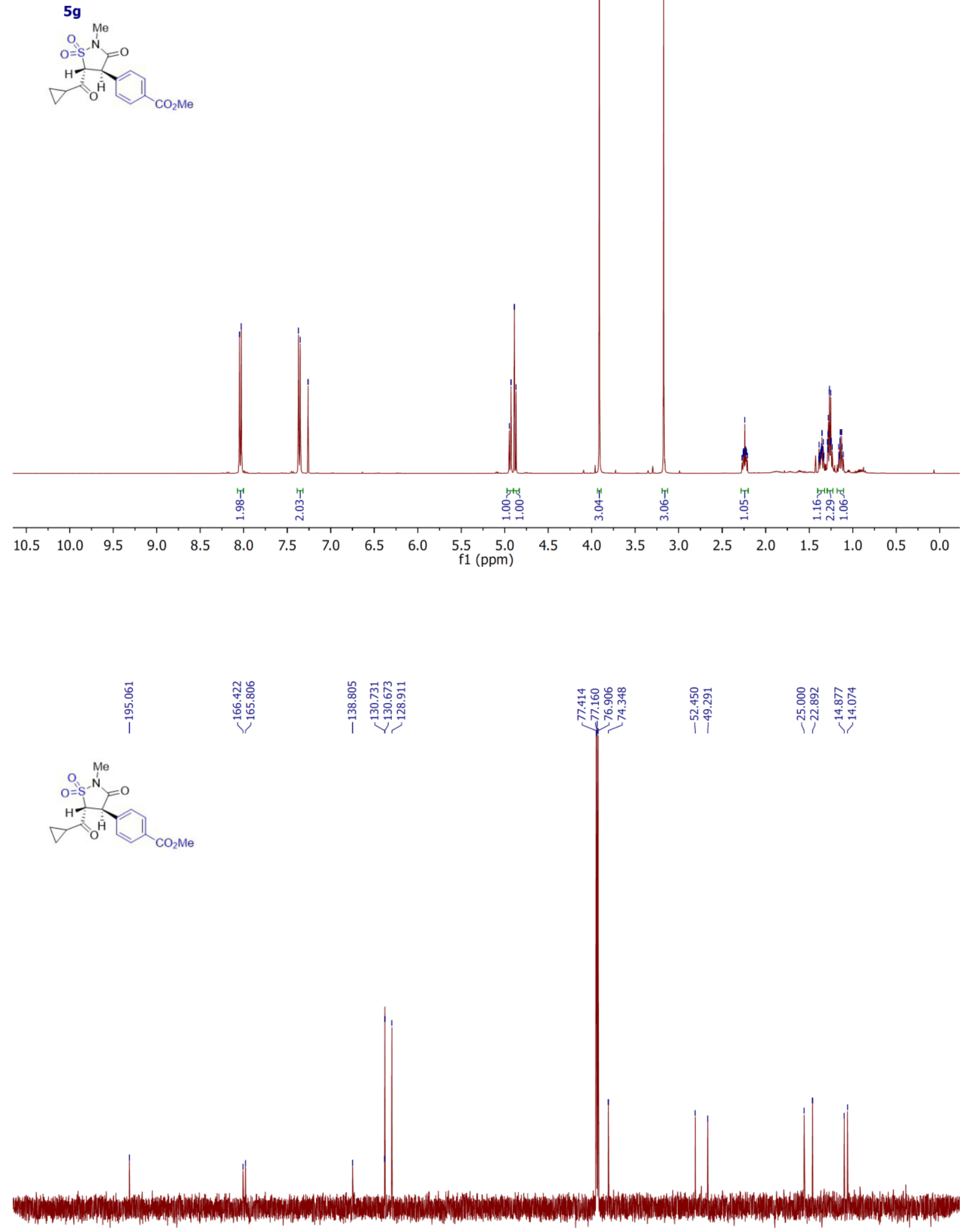

$\begin{array}{lllllllllllllllllllllllllll}220 & 210 & 200 & 190 & 180 & 170 & 160 & 150 & 140 & 130 & 120 & \begin{array}{c}110 \\ \mathrm{f} 1(\mathrm{ppm})\end{array} & 90 & 80 & 70 & 60 & 50 & 40 & 30 & 20 & 10 & 0 & -10\end{array}$ 


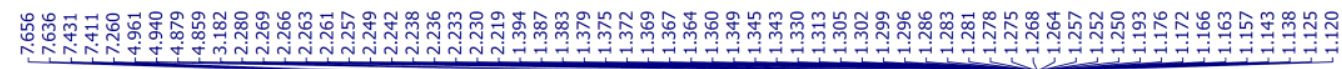

5h

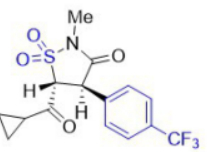

$\nabla \mathrm{O}^{\mathrm{H}} \mathrm{CF}_{3}$
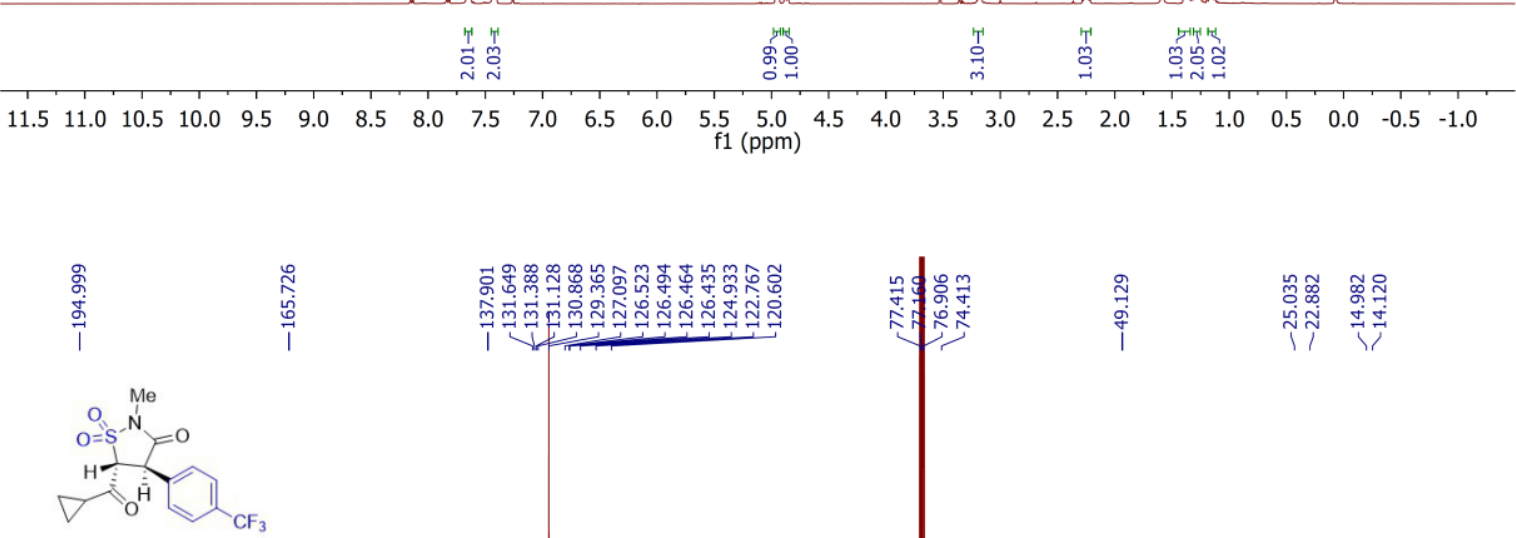

$\begin{array}{lllllllllllllllllllll}200 & 190 & 180 & 170 & 160 & 150 & 140 & 130 & 120 & 110 \underset{\mathrm{f} 1(\mathrm{ppm})}{100} & 90 & 80 & 70 & 60 & 50 & 40 & 30 & 20 & 10 & 0\end{array}$
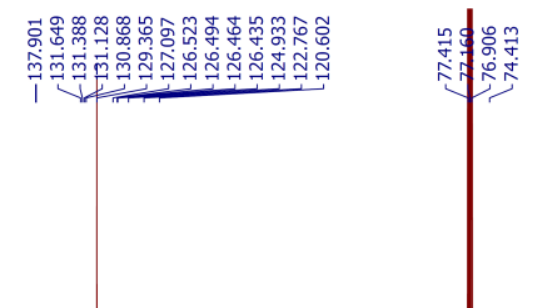

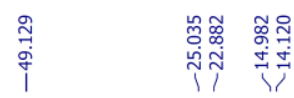

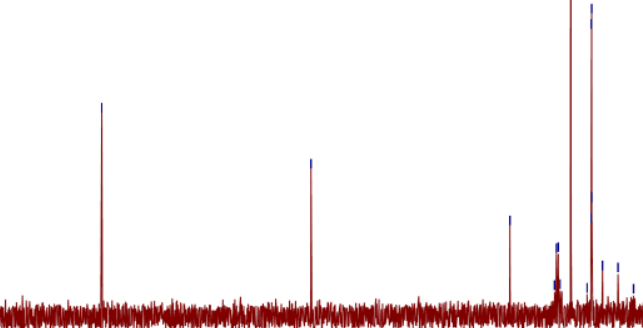


${ }^{19} \mathrm{~F} \mathrm{NMR}\left(376 \mathrm{MHz}, \mathrm{CDCl}_{3}\right) \delta-62.88$
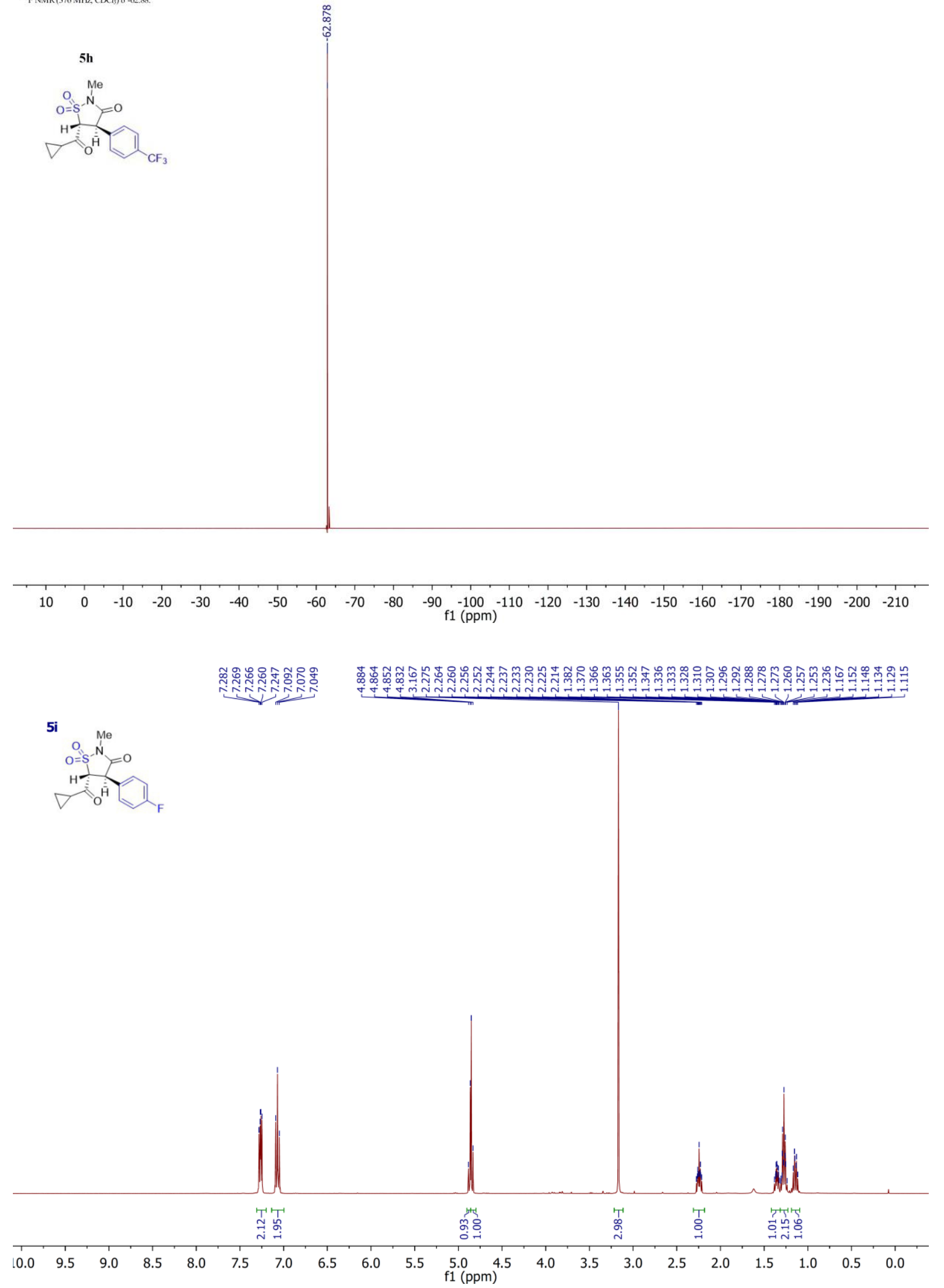
$5 i$
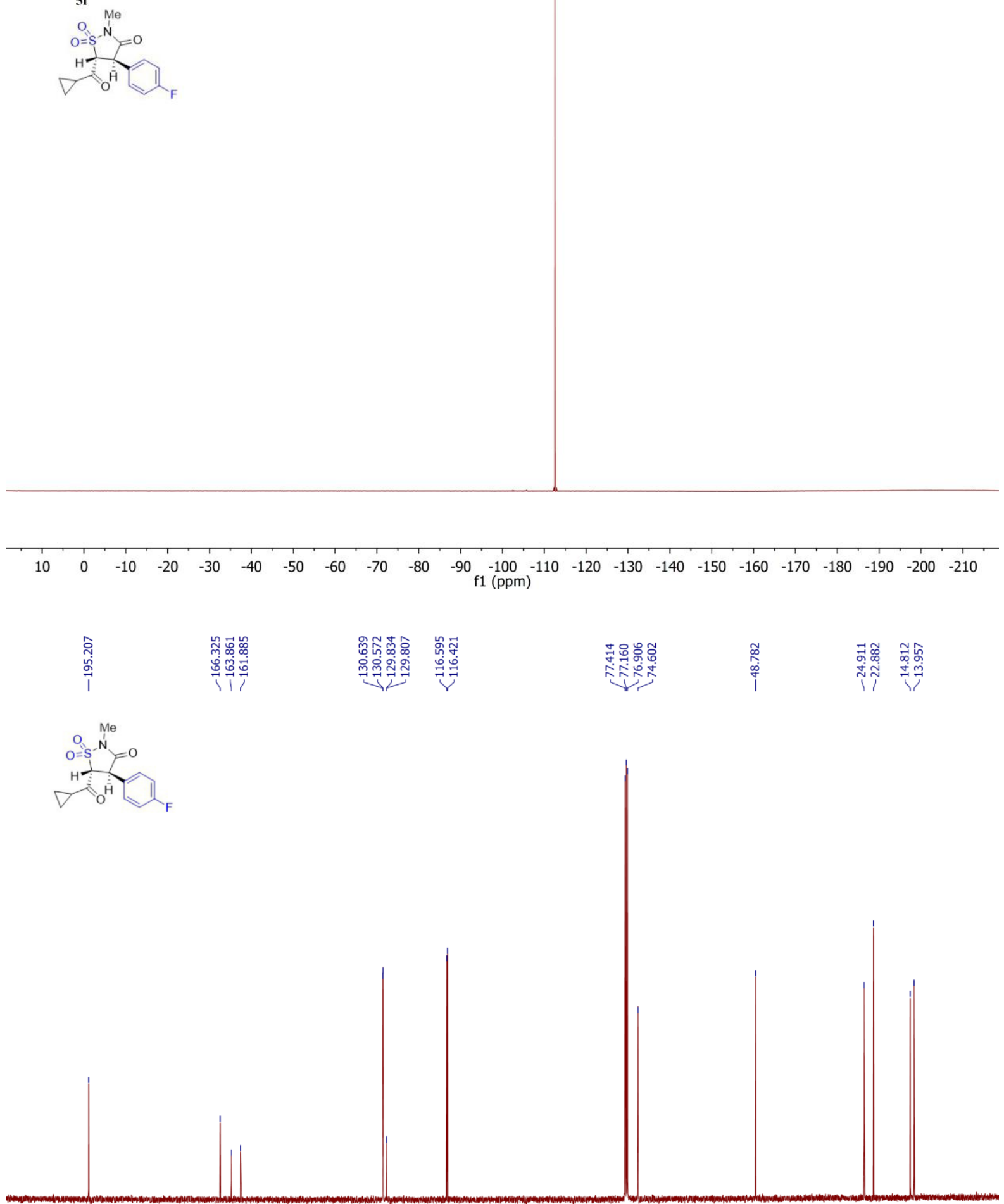

$\begin{array}{lllllllllll}210 & 200 & 190 & 180 & 170 & 160 & 150 & 140 & 130 & 120 & 110 \\ \mathrm{f} 1(\mathrm{ppm})\end{array}$

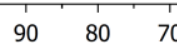




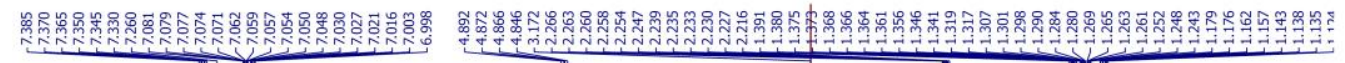
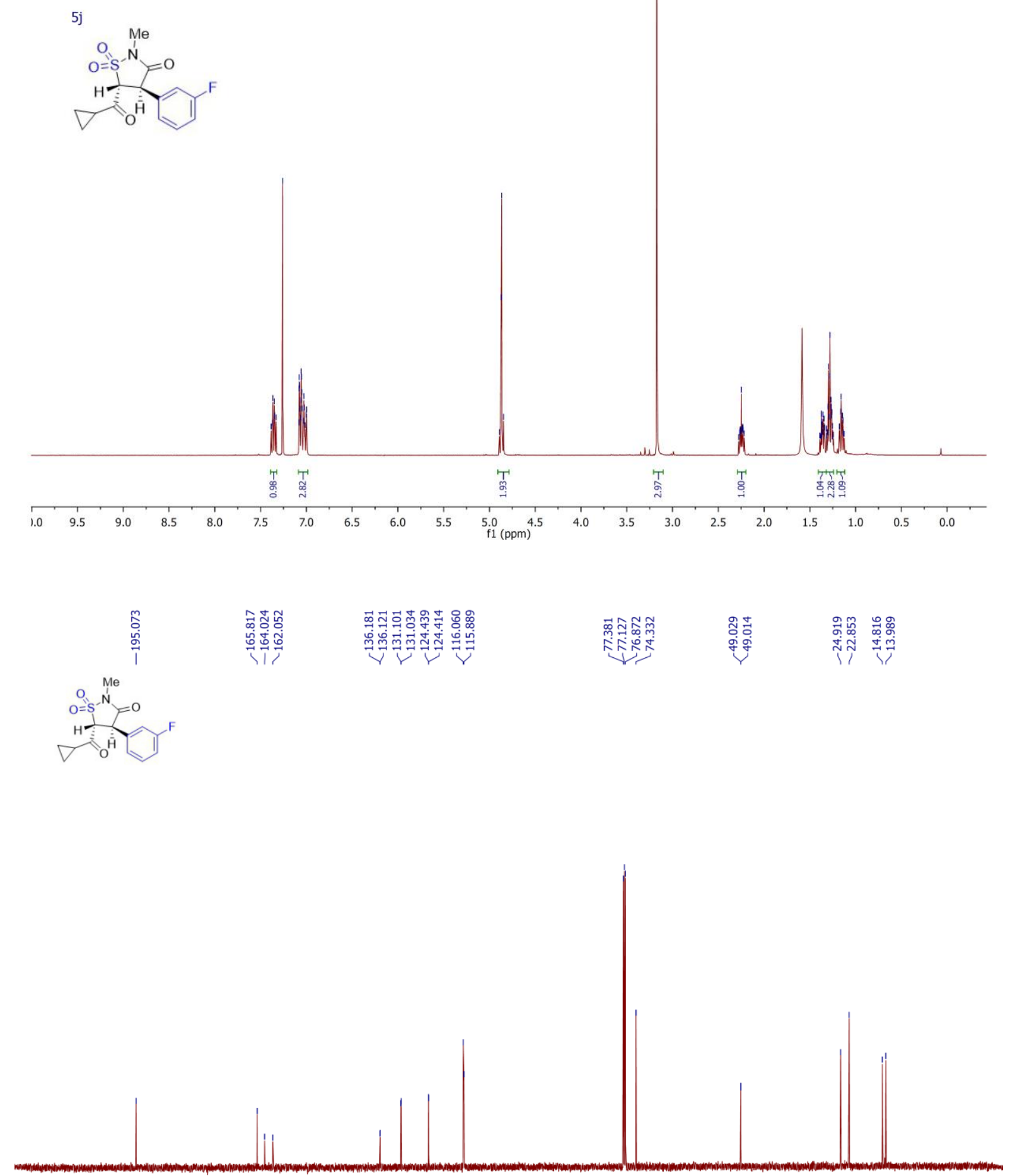

$\begin{array}{lllllllllllllllllllllll}220 & 210 & 200 & 190 & 180 & 170 & 160 & 150 & 140 & 130 & 120 & \begin{array}{c}110 \\ \mathrm{f} 1(\mathrm{ppm})\end{array} & 90 & 80 & 70 & 60 & 50 & 40 & 30 & 20 & 10 & 0 & -10\end{array}$ 


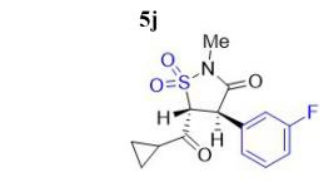

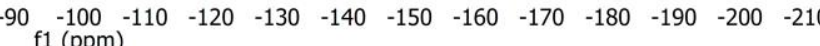

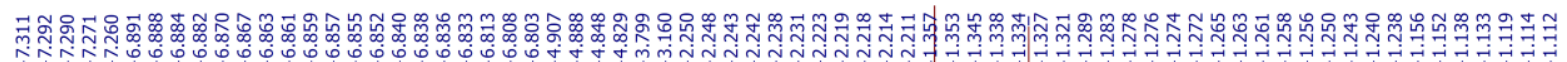
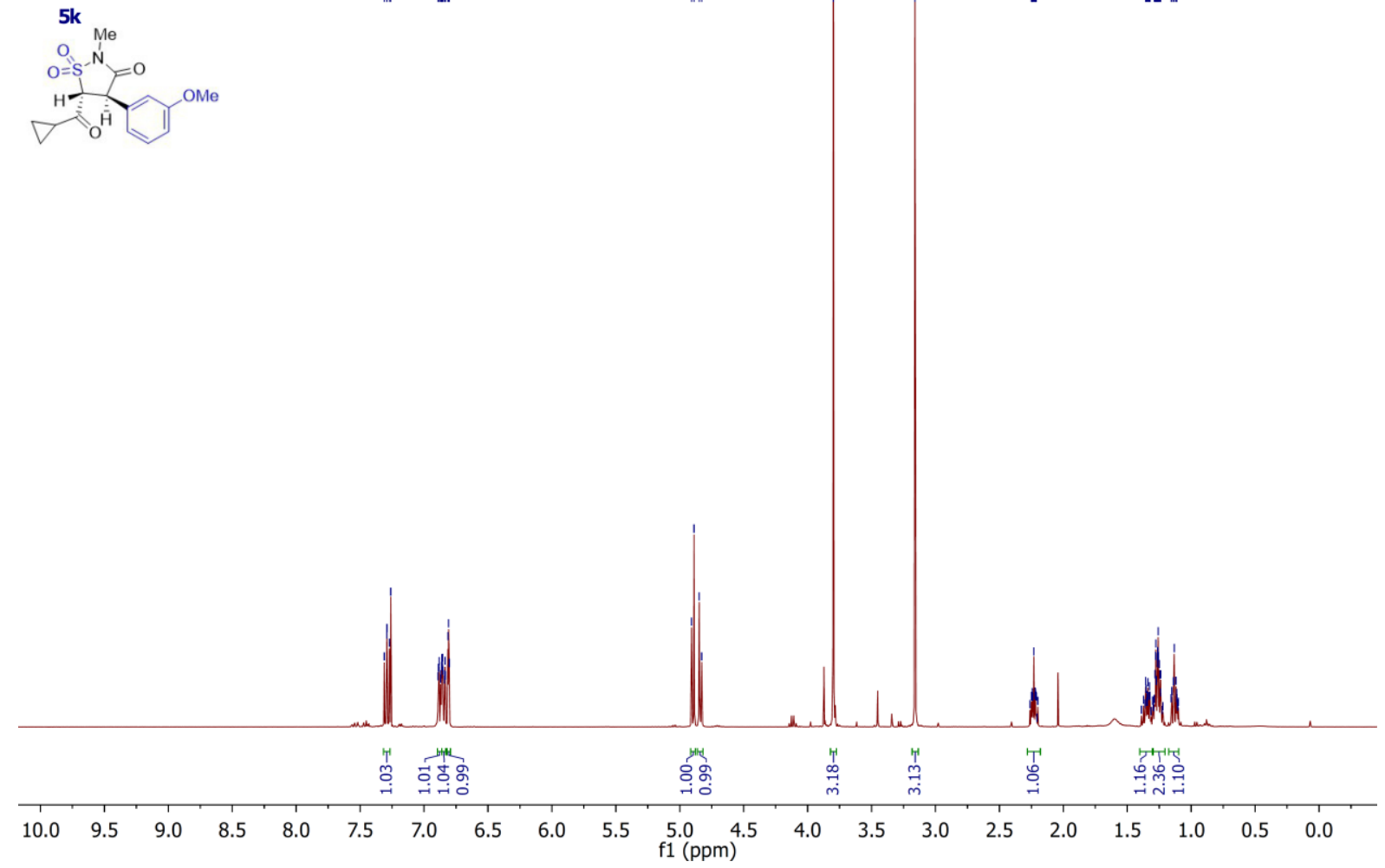

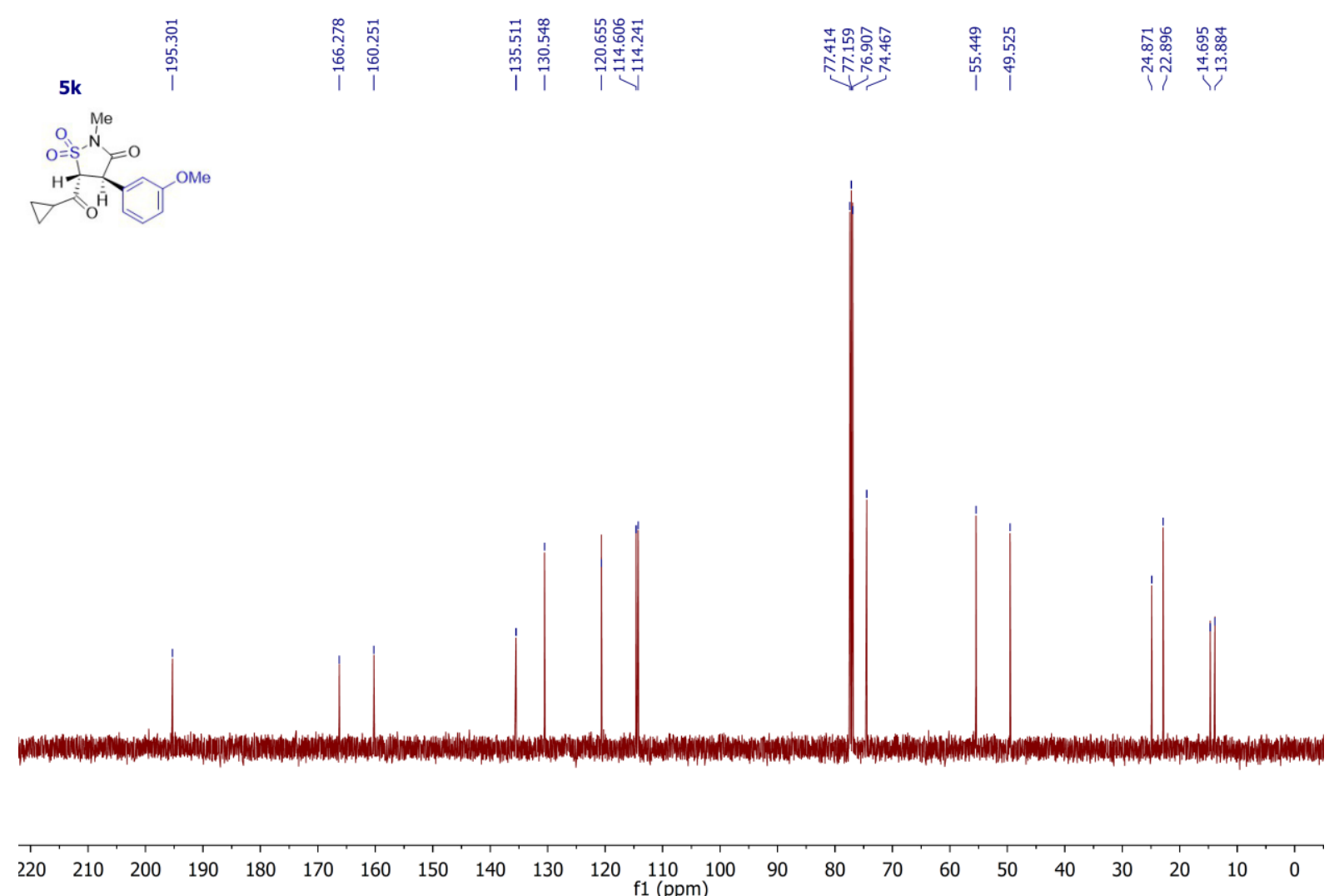

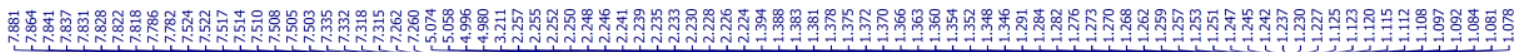
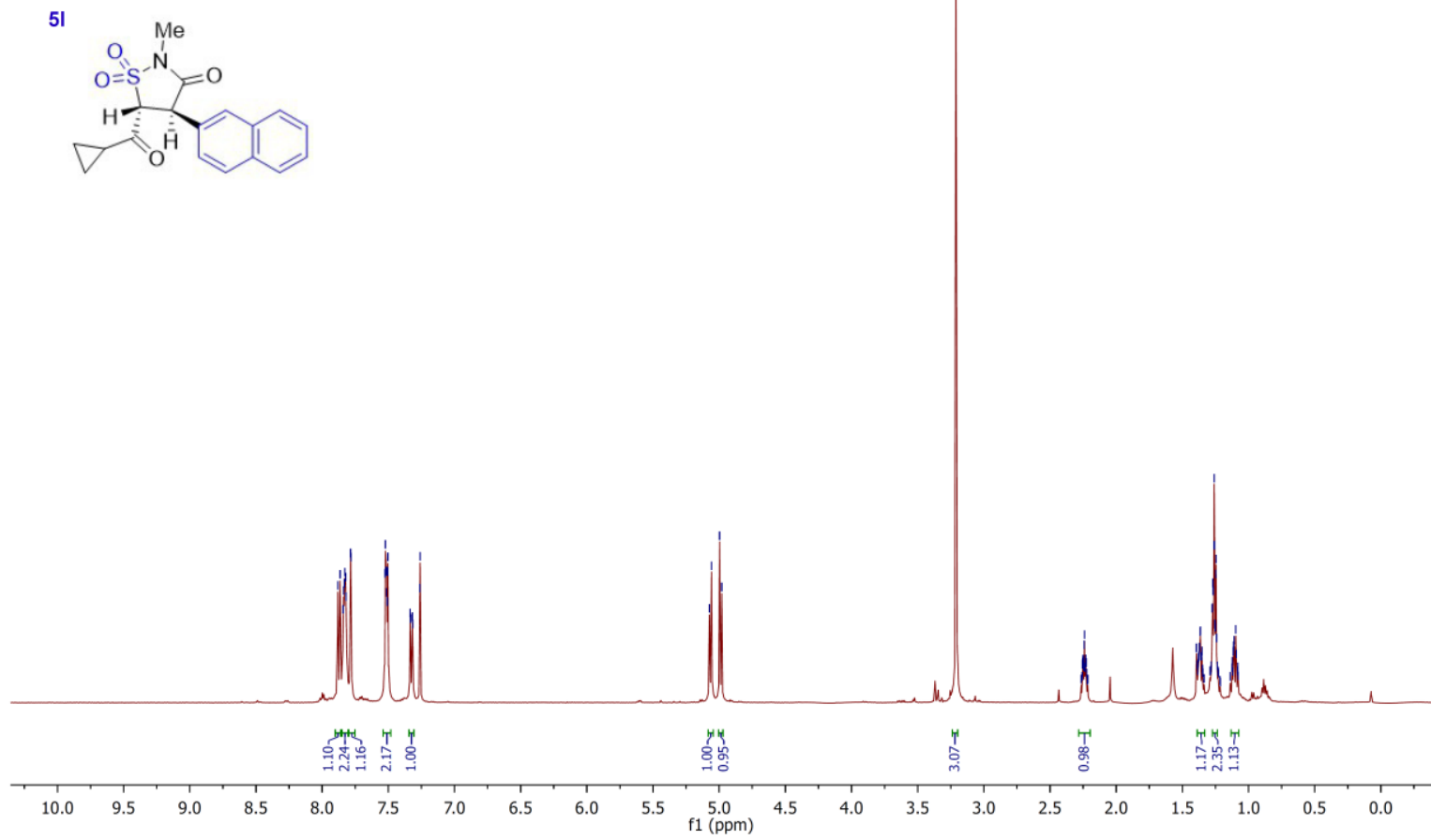


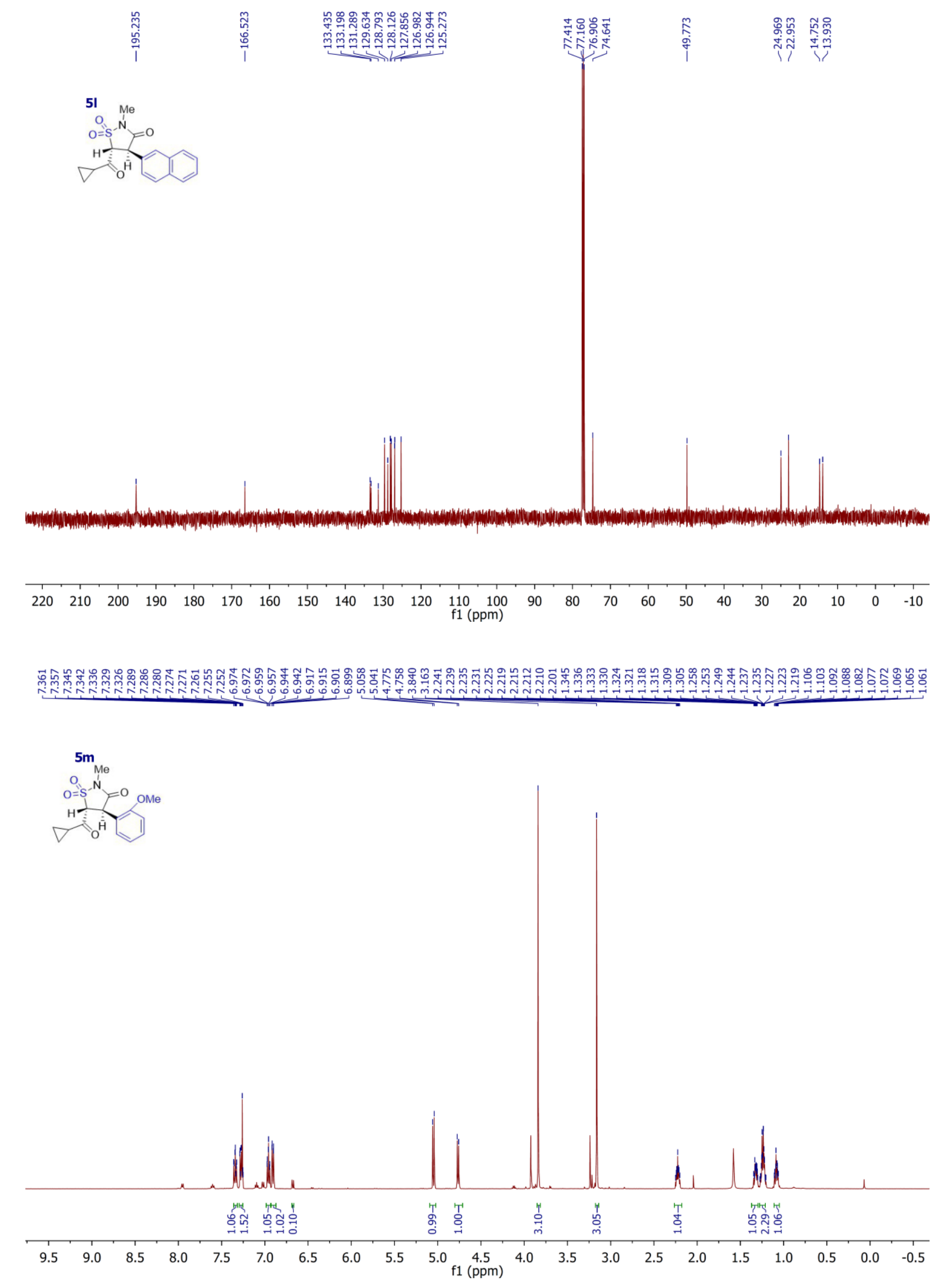




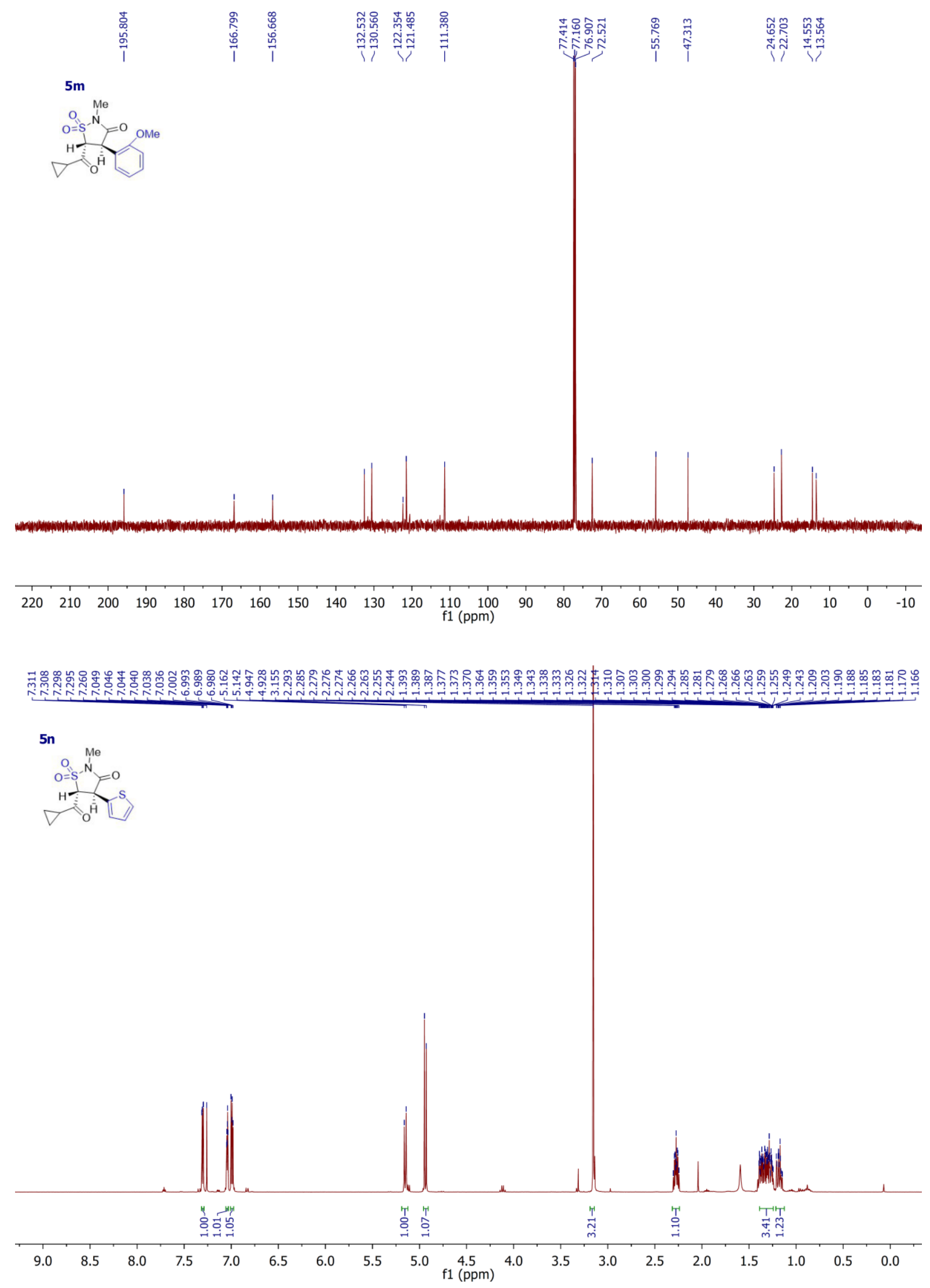




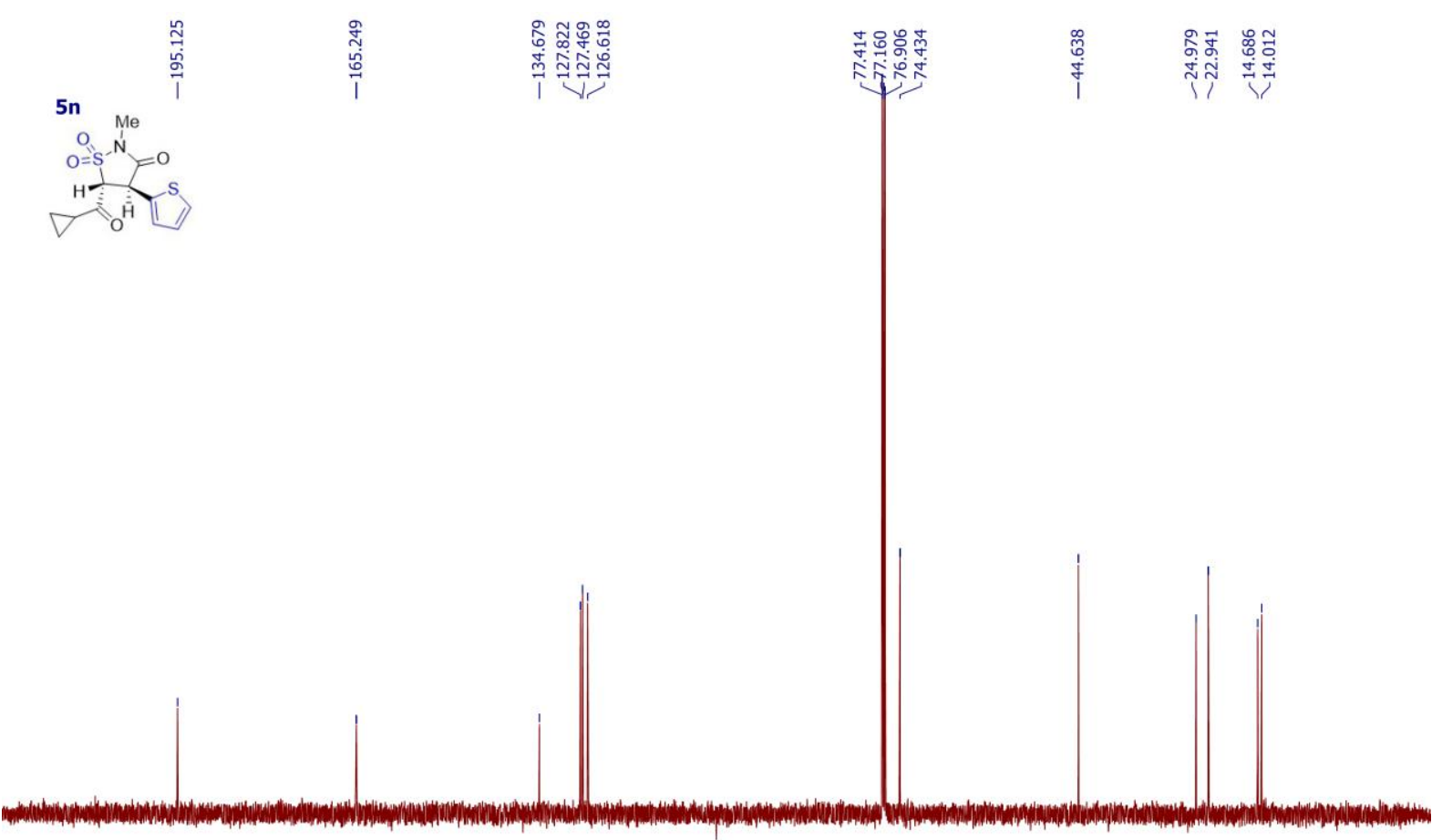

$\begin{array}{lllllllllllllllllllllllll}220 & 210 & 200 & 190 & 180 & 170 & 160 & 150 & 140 & 130 & 120 & 110 & 100 & 90 & 80 & 70 & 60 & 50 & 40 & 30 & 20 & 10 & 0 & -10\end{array}$

养的

50
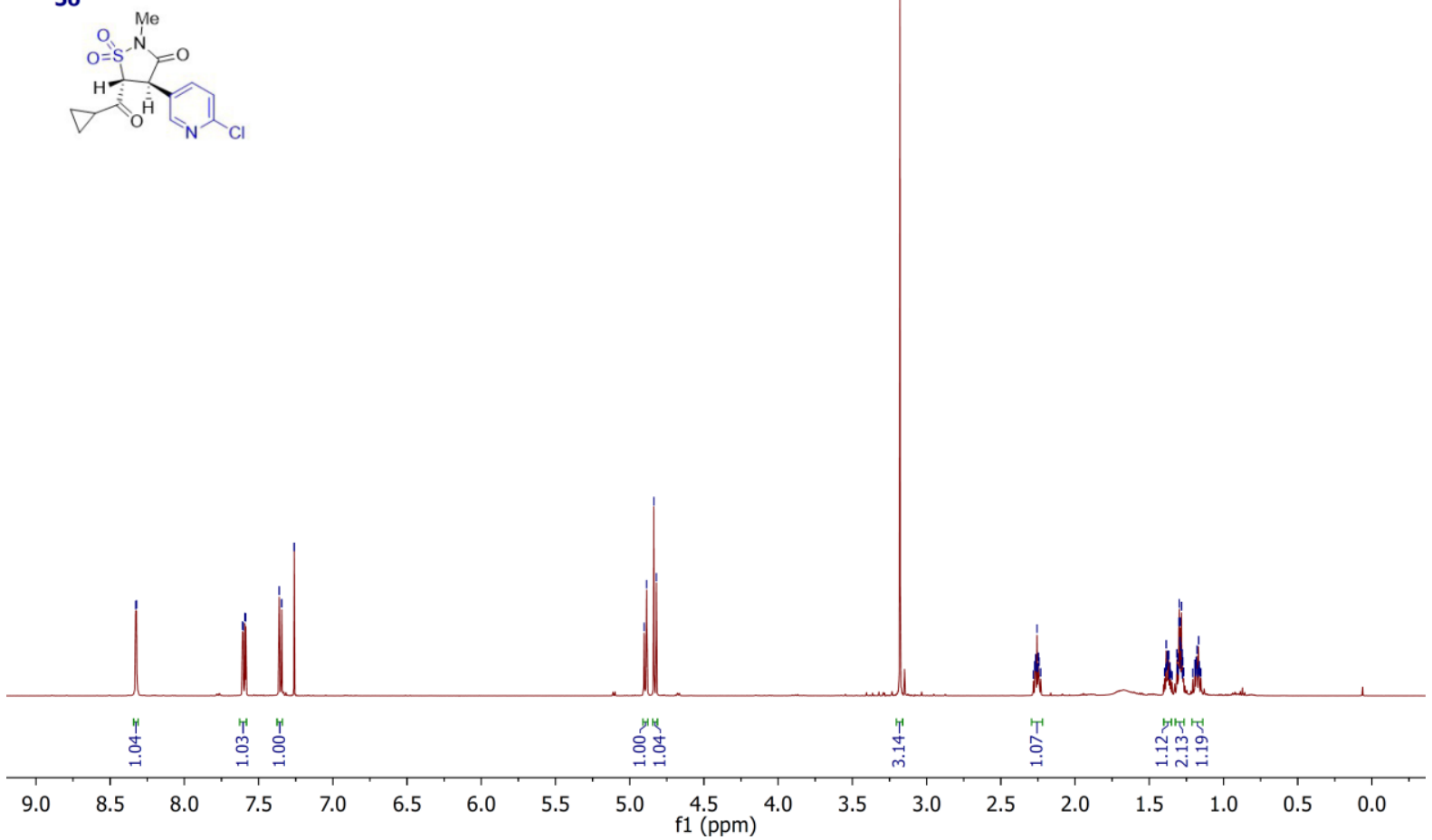

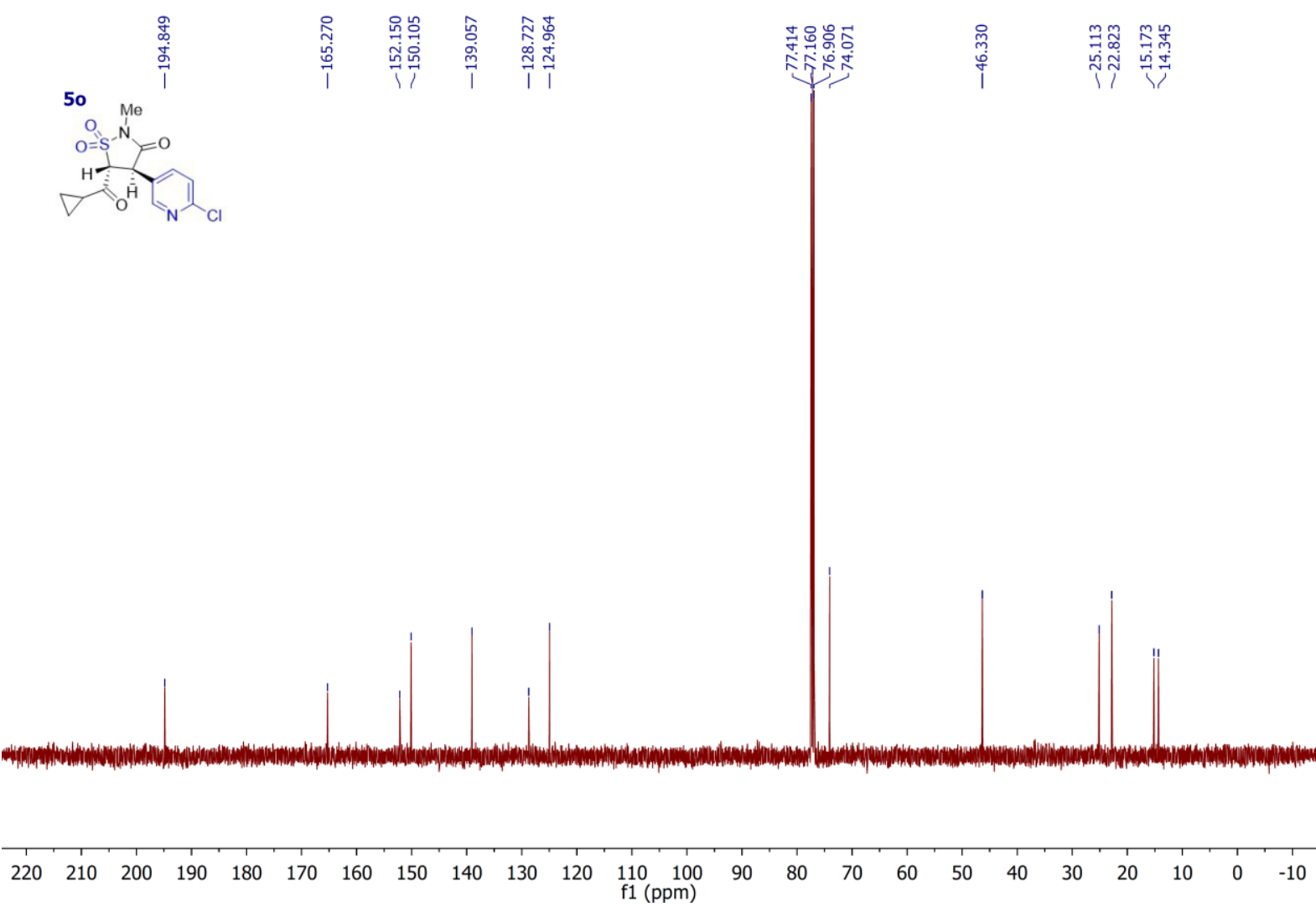

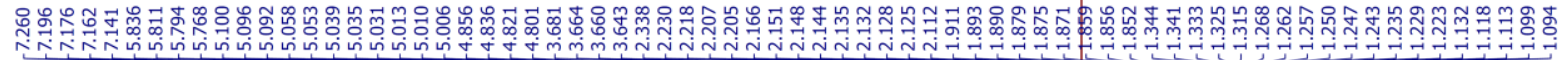

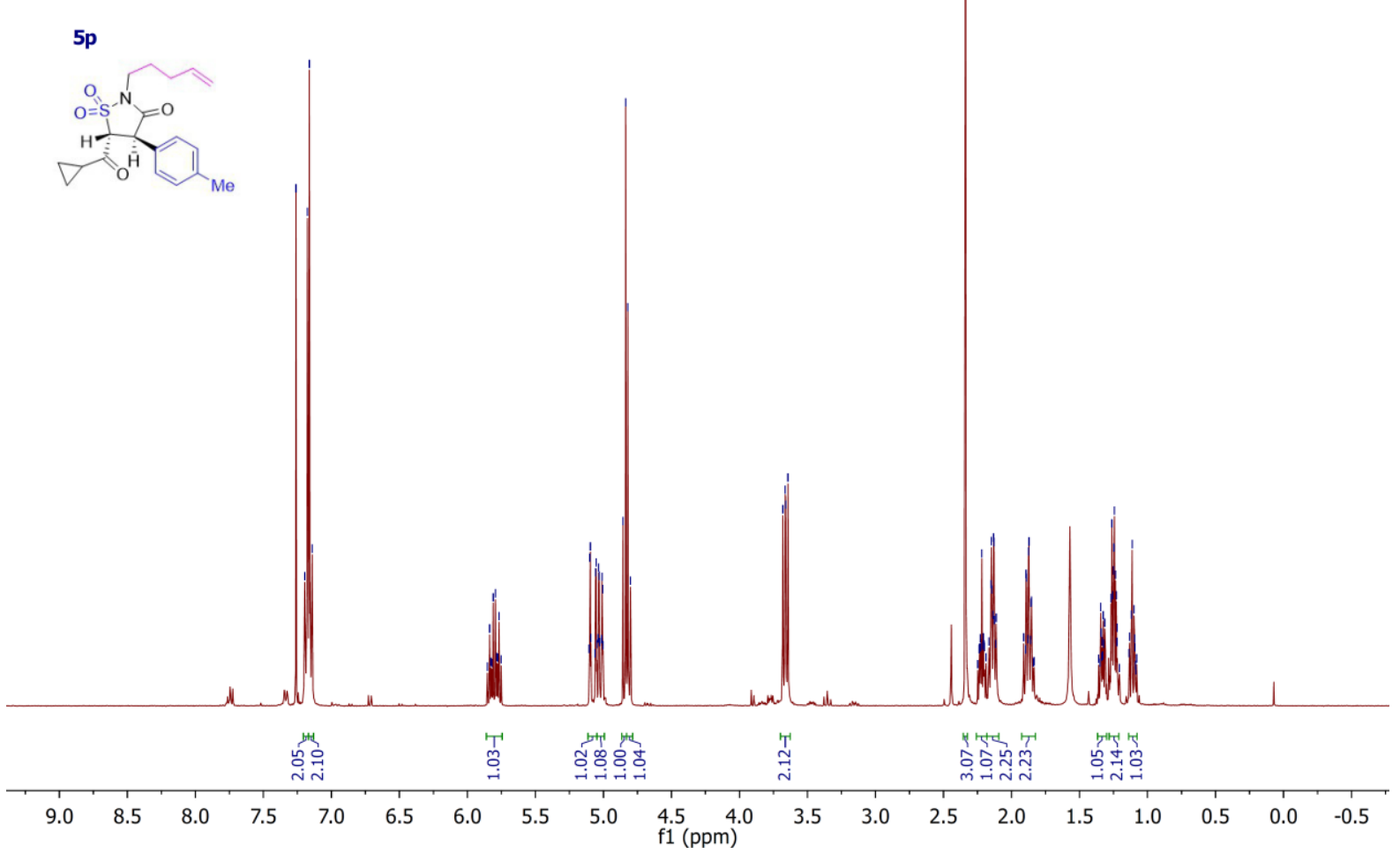




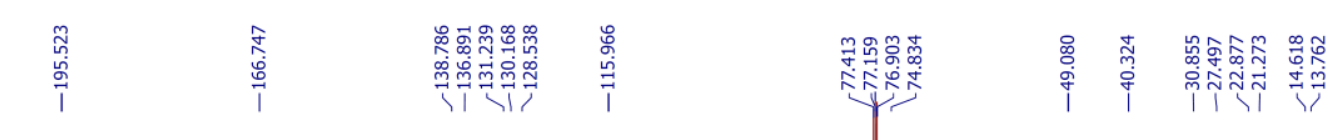

$5 p$
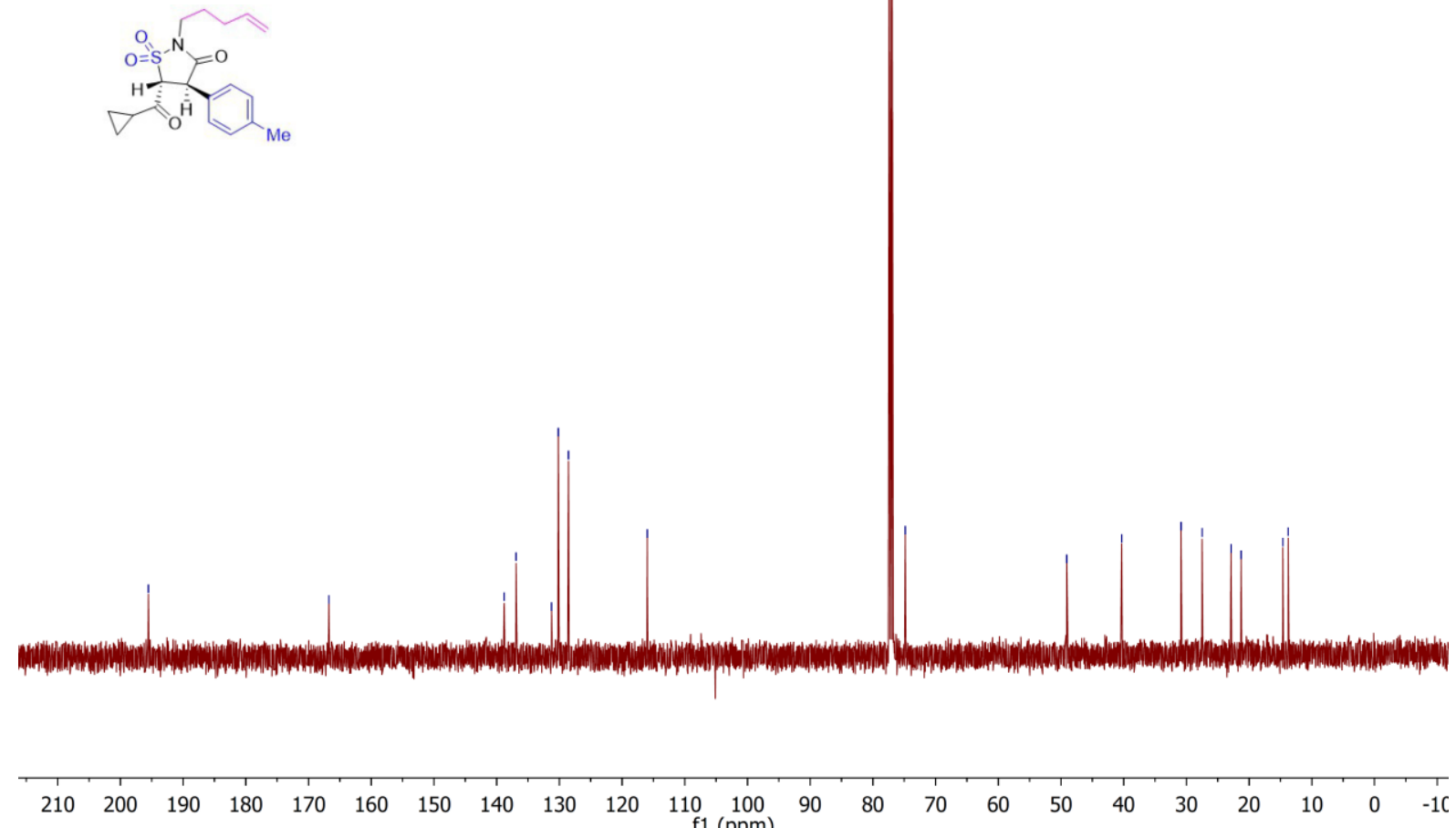

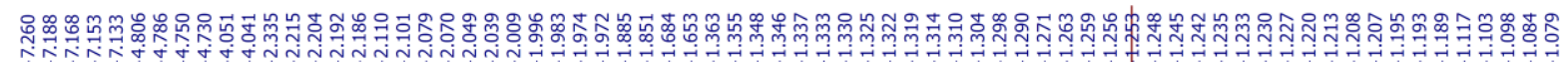

5q
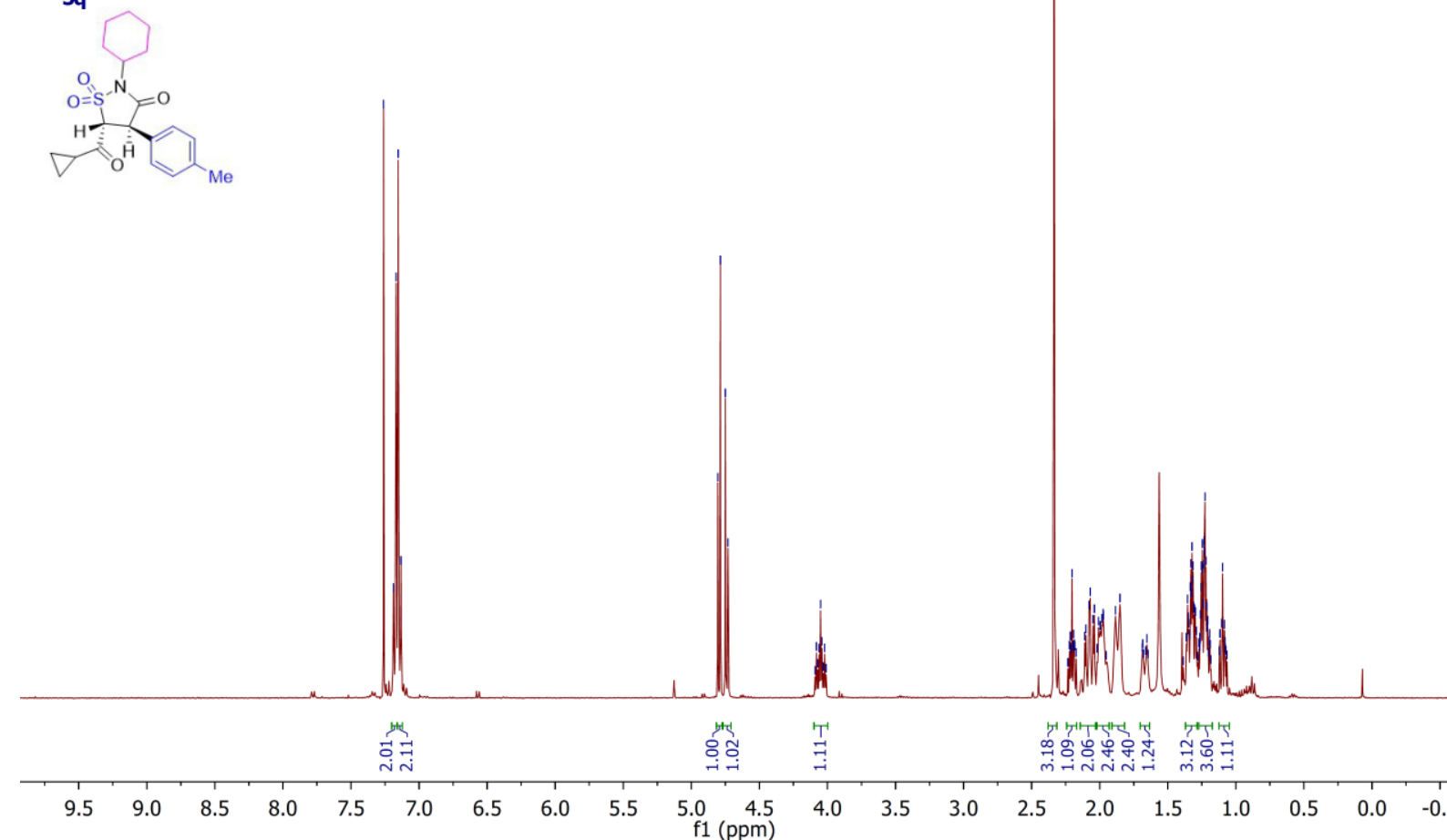


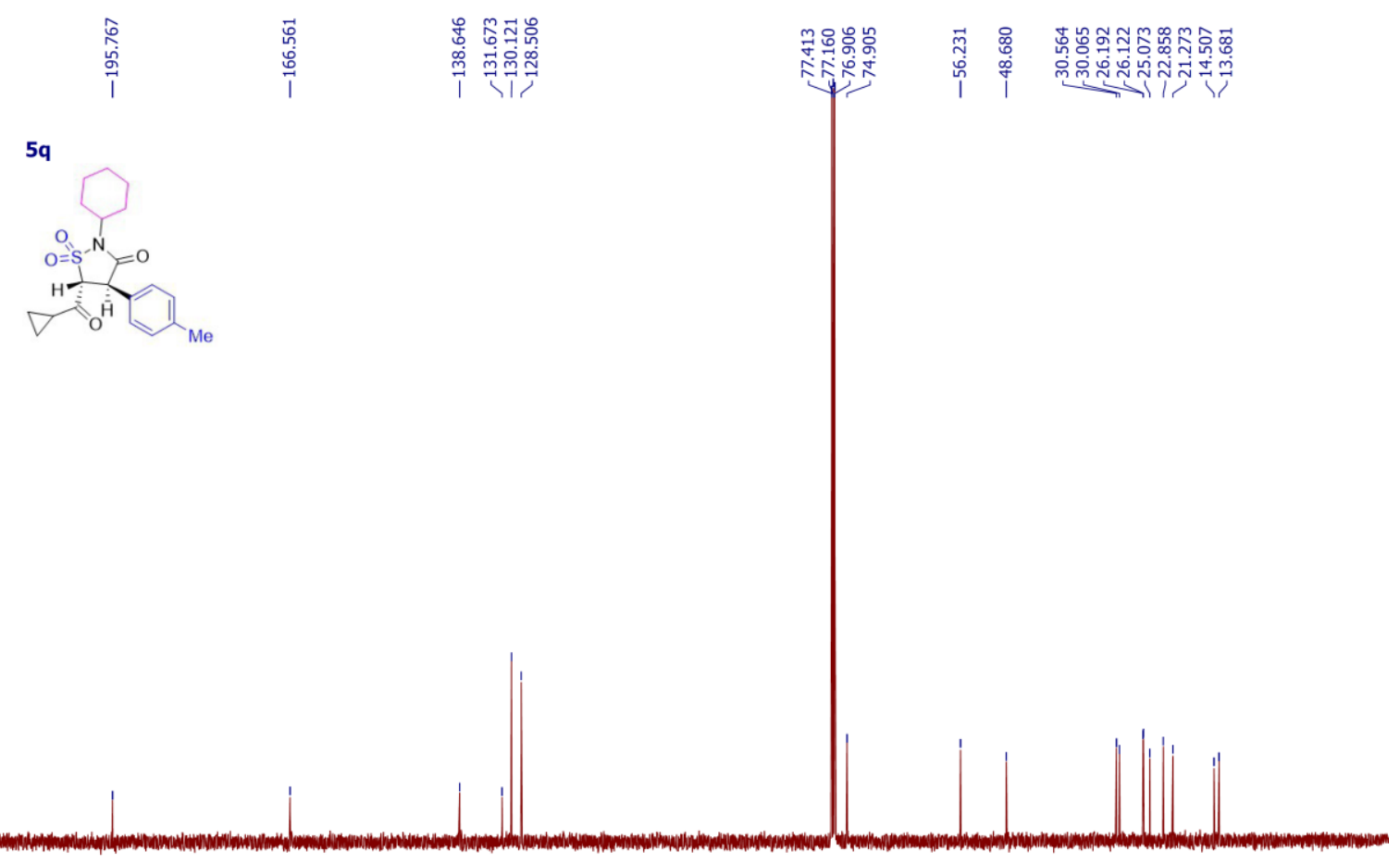

$\begin{array}{lllllllllllllllllllllll}220 & 210 & 200 & 190 & 180 & 170 & 160 & 150 & 140 & 130 & 120 & \begin{array}{c}110 \\ \mathrm{f} 1(\mathrm{ppm})\end{array} & 90 & 80 & 70 & 60 & 50 & 40 & 30 & 20 & 10 & 0 & -10\end{array}$

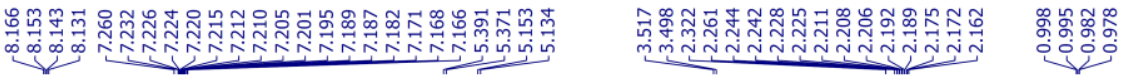
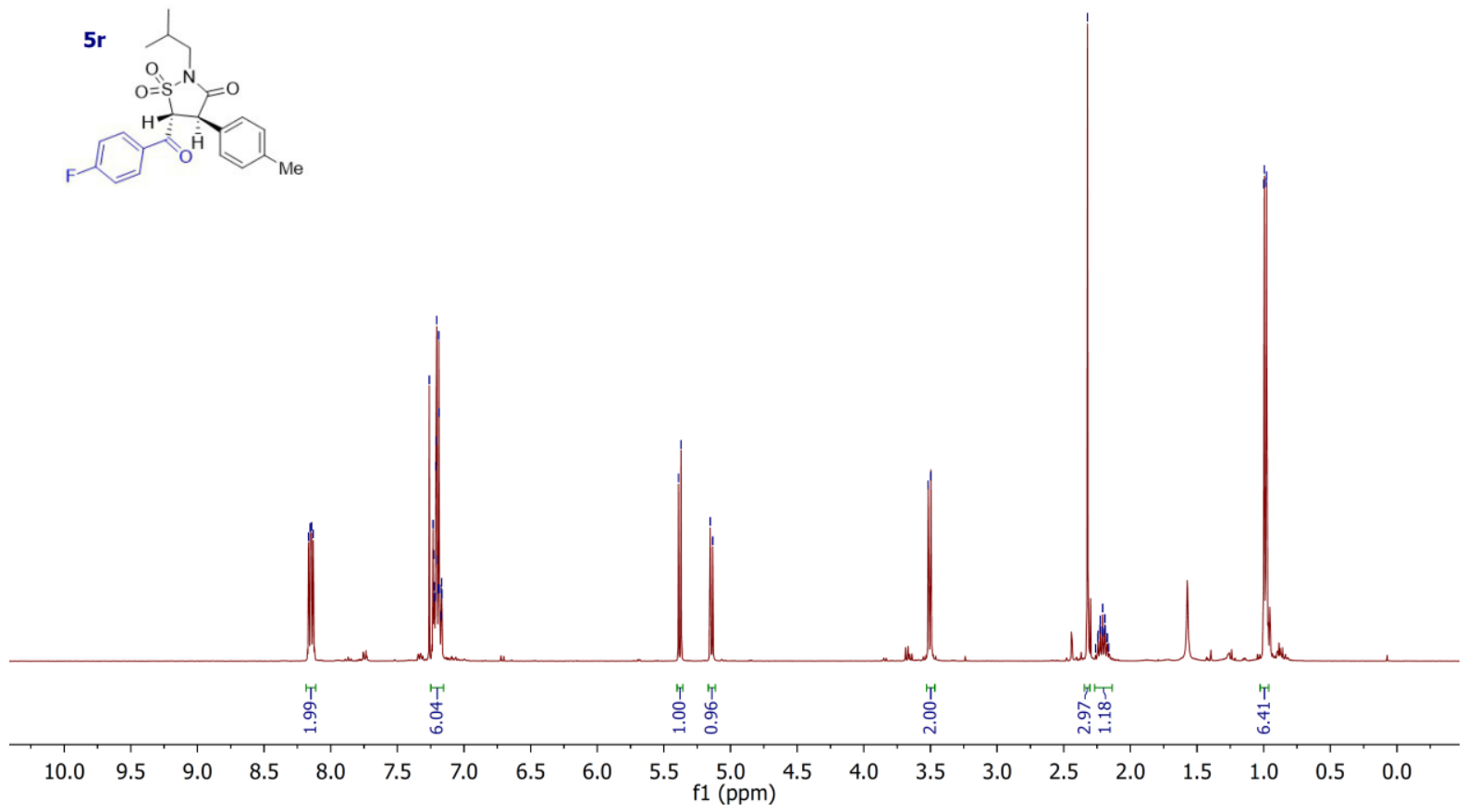

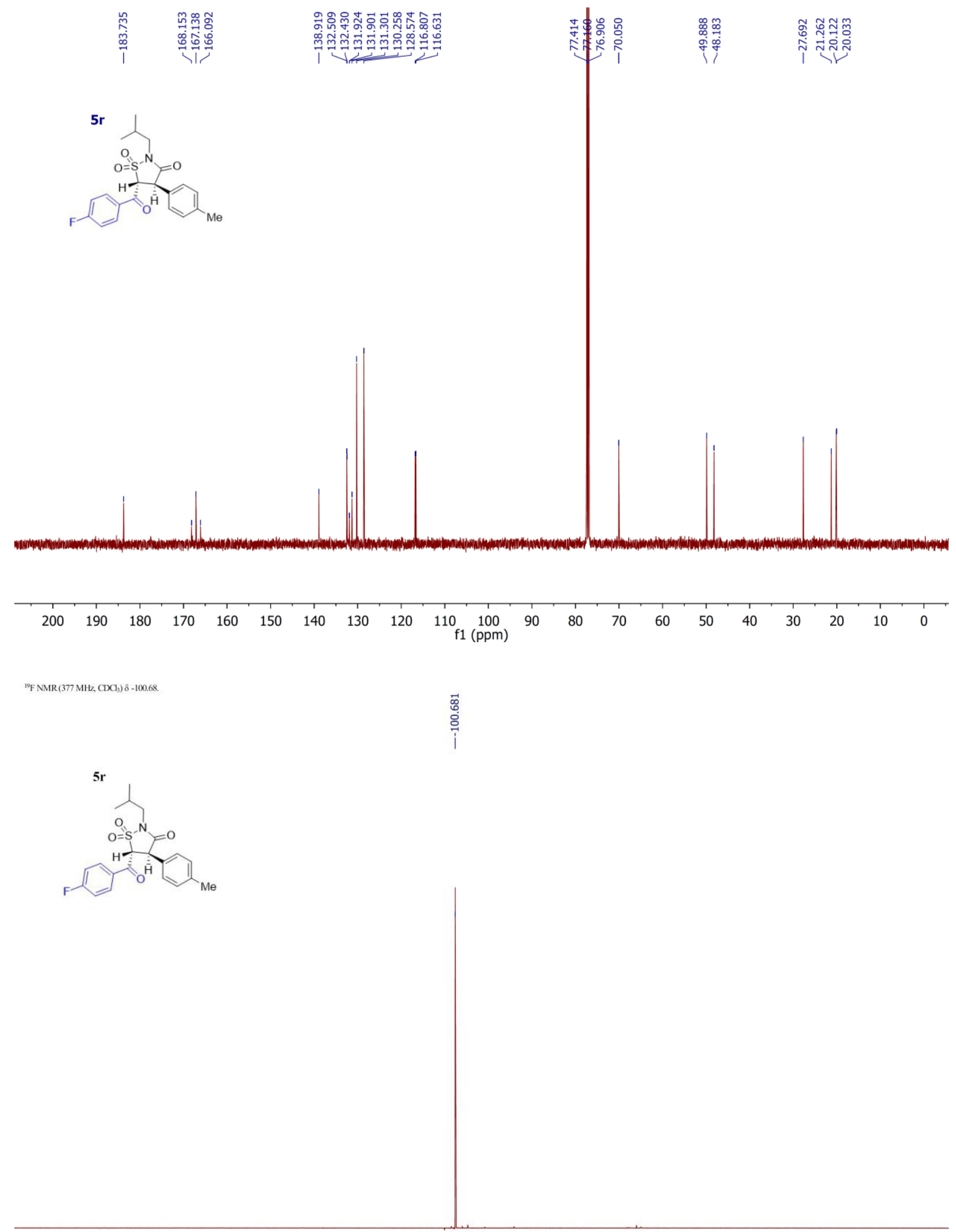

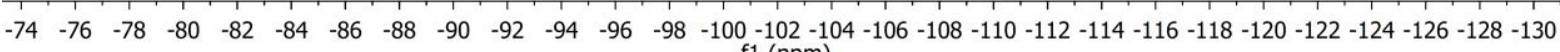
f1 (ppm) 
Don

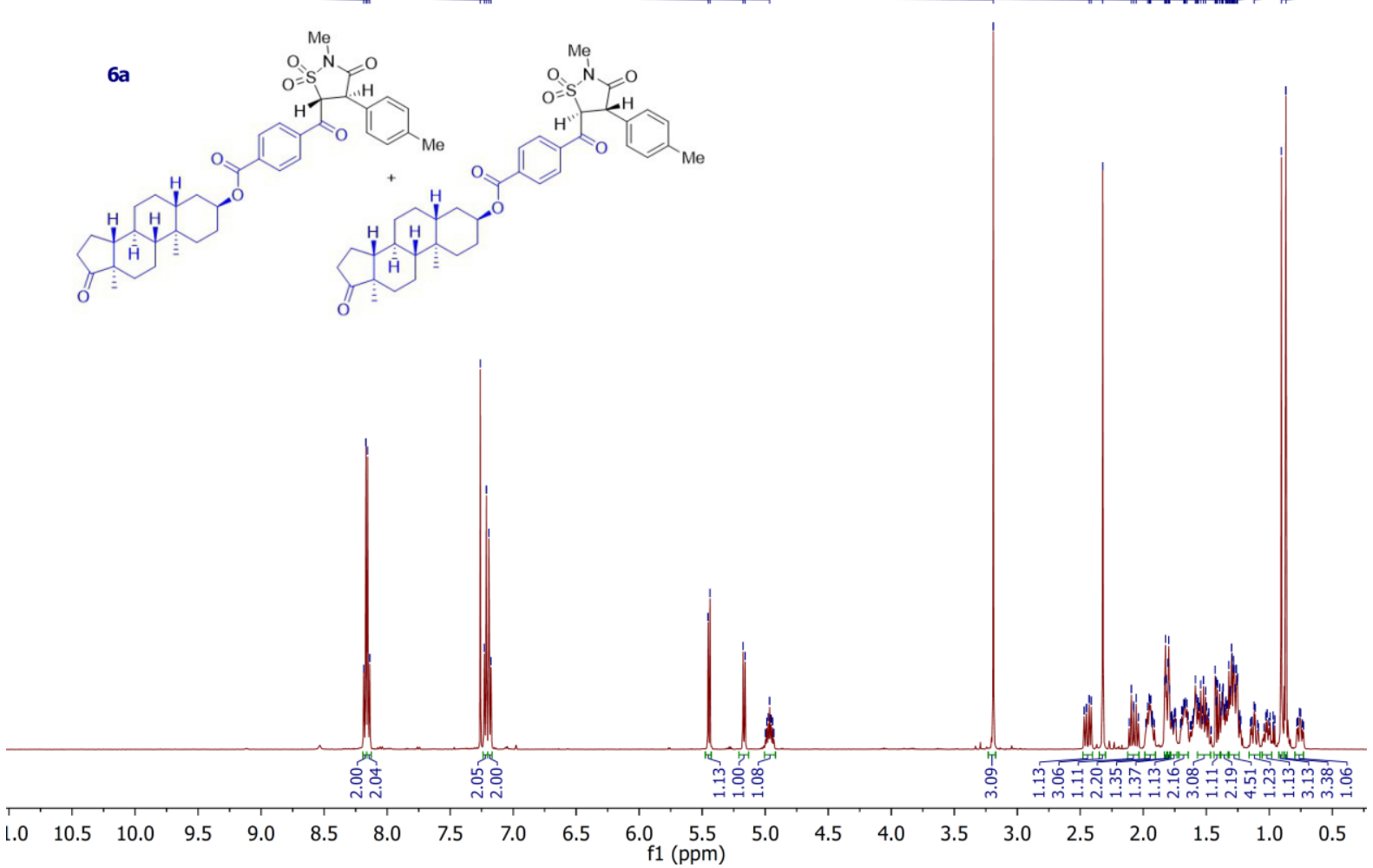

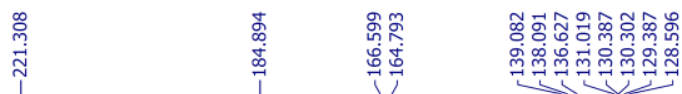

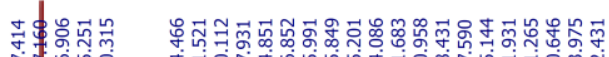

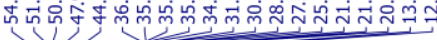
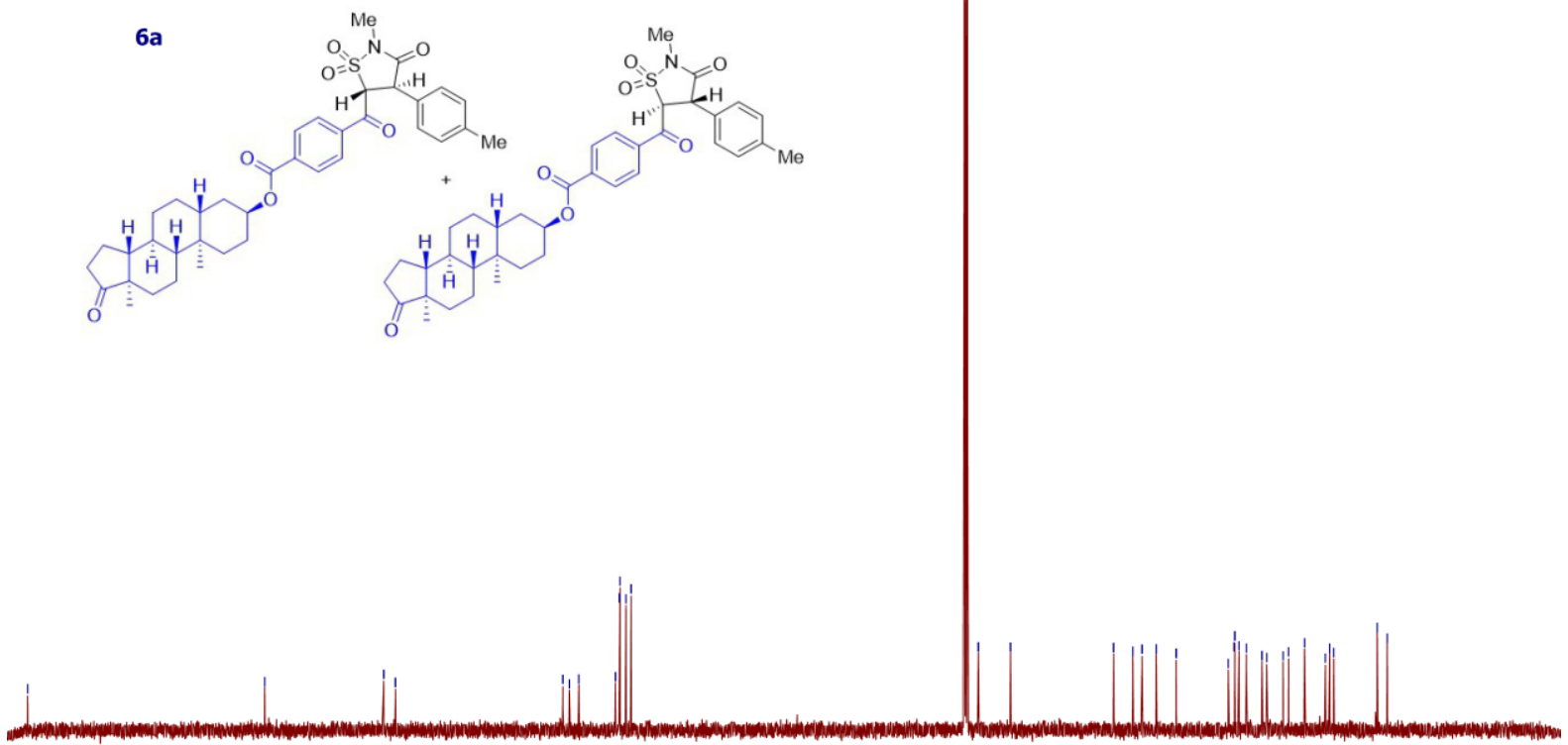

$\begin{array}{llllllllllllllllllllllll}220 & 210 & 200 & 190 & 180 & 170 & 160 & 150 & 140 & 130 & 120 & 110 & 100 & 90 & 80 & 70 & 60 & 50 & 40 & 30 & 20 & 10 & 0 & -10\end{array}$ 


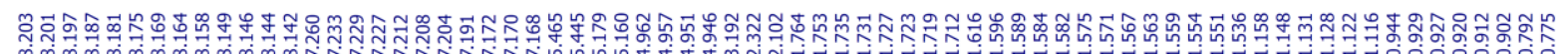

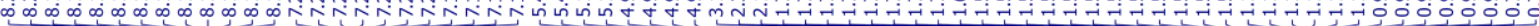

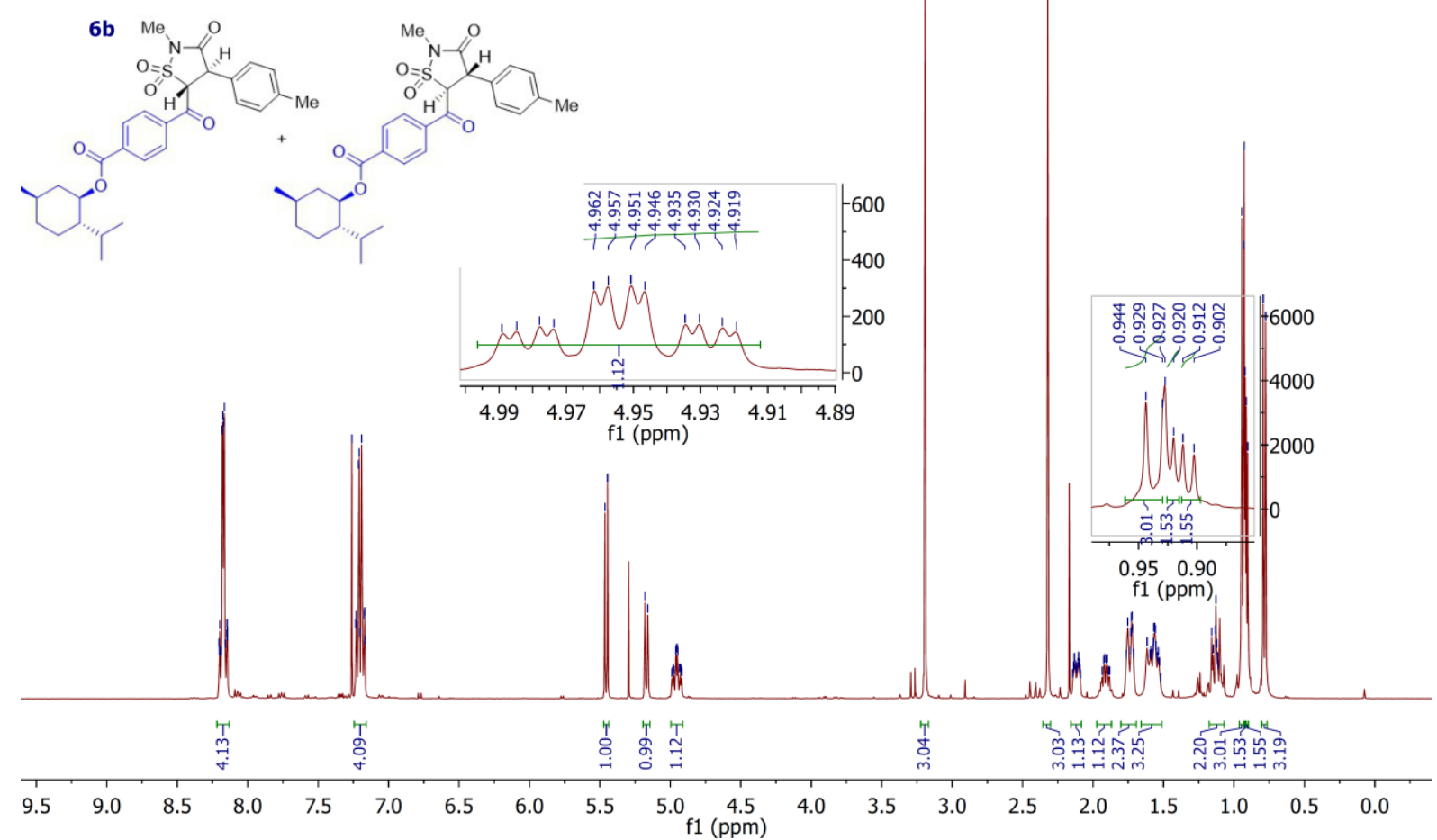

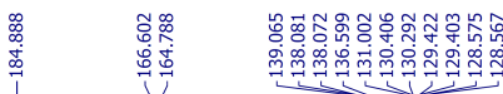

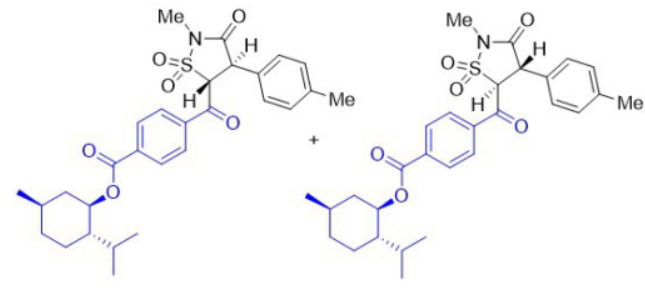

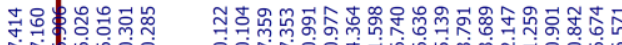

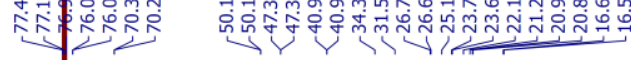

$\begin{array}{lllllllllll}210 & 200 & 190 & 180 & 170 & 160 & 150 & 140 & 130 & 120 & \begin{array}{c}110 \\ \mathrm{f} 1(\mathrm{ppm})\end{array}\end{array}$

$90 \quad 80$

$80 \quad 60 \quad 50$

$\begin{array}{lllll}40 & 30 & 20 & 10 & 0\end{array}$ 


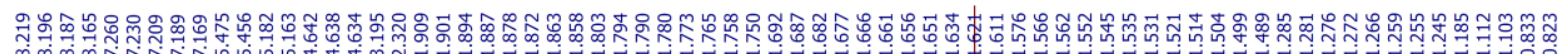
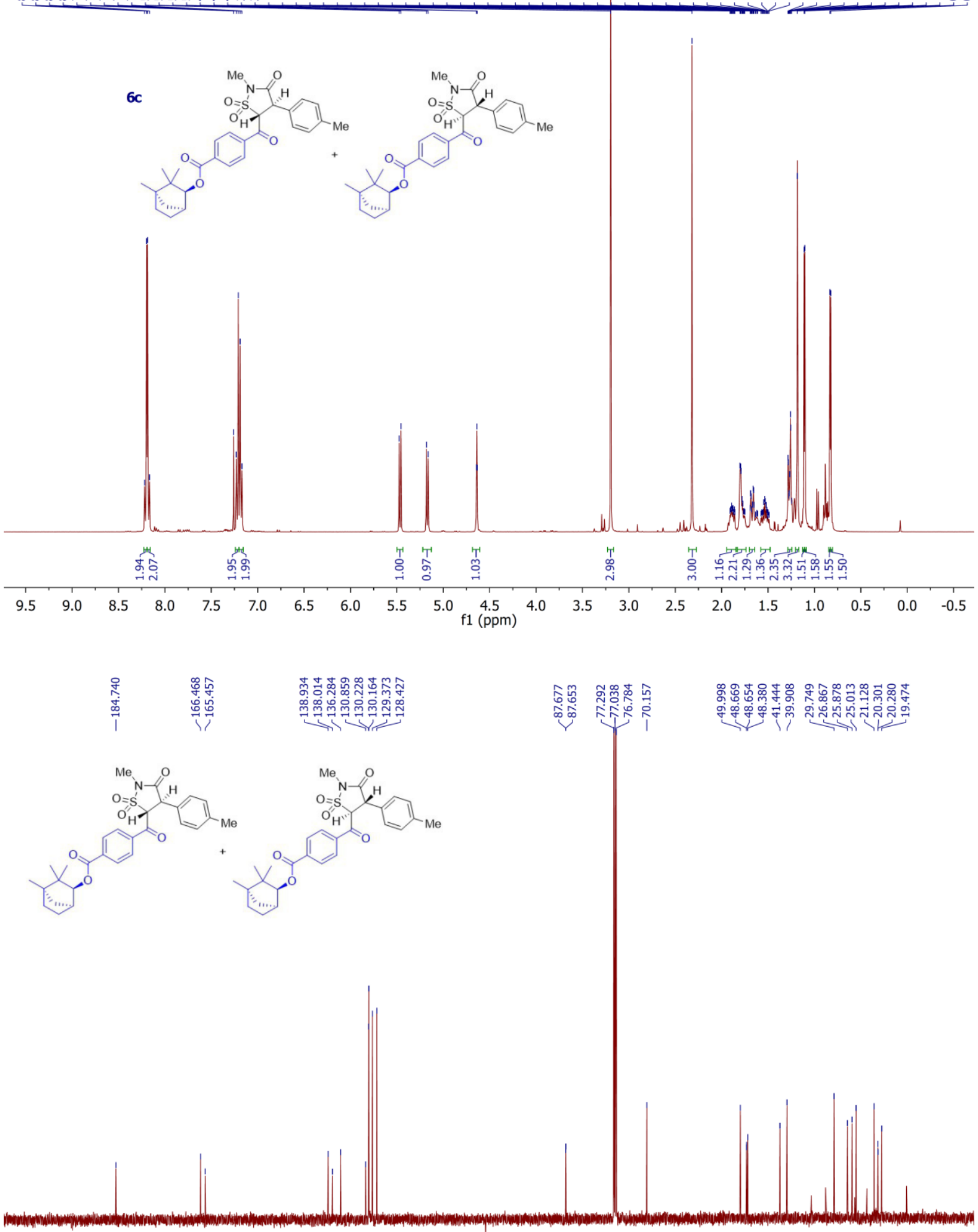

$\begin{array}{lllllllllll}200 & 190 & 180 & 170 & 160 & 150 & 140 & 130 & 120 & \begin{array}{l}110 \\ \mathrm{f} 1(\mathrm{ppm})\end{array}\end{array}$

$\begin{array}{llllllllll}90 & 80 & 70 & 60 & 50 & 40 & 30 & 20 & 10 & \mathrm{C}\end{array}$ 


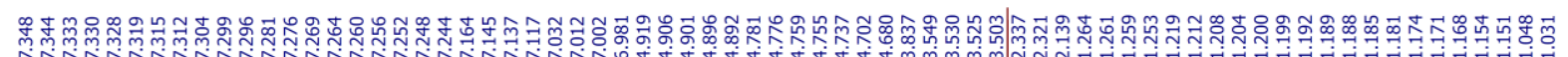
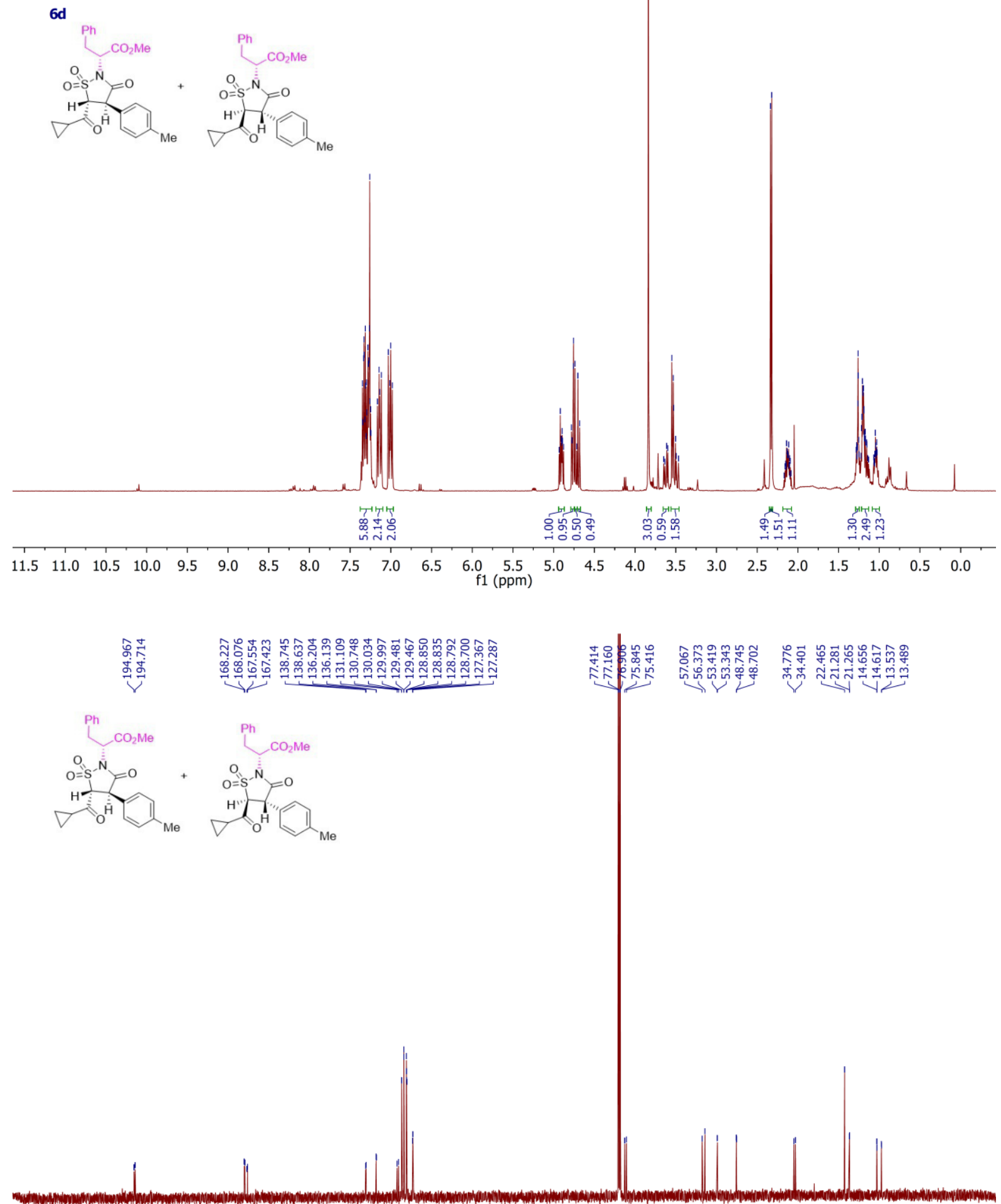

$\begin{array}{lllllllllllllllllllllllll}220 & 210 & 200 & 190 & 180 & 170 & 160 & 150 & 140 & 130 & 120 & \begin{array}{l}110 \\ \mathrm{f} 1(\mathrm{ppm})\end{array} & 90 & 80 & 70 & 60 & 50 & 40 & 30 & 20 & 10 & 0 & -10\end{array}$ 

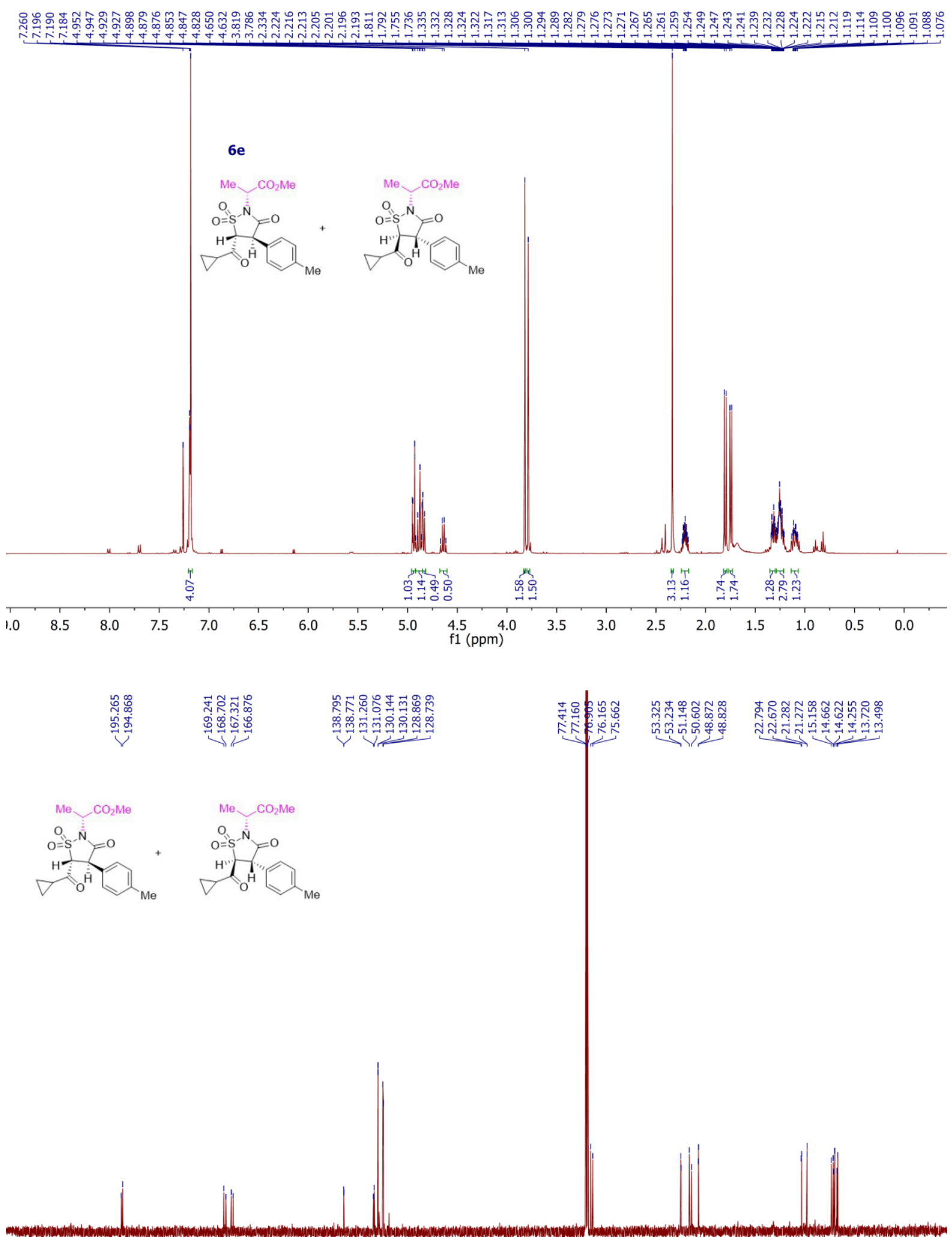

$\begin{array}{llllllllllllllllllllllllllll}220 & 210 & 200 & 190 & 180 & 170 & 160 & 150 & 140 & 130 & 120 & \begin{array}{l}110 \\ \mathrm{f} 1(\mathrm{ppm})\end{array} & 90 & 80 & 70 & 60 & 50 & 40 & 30 & 20 & 10 & 0 & -10\end{array}$ 


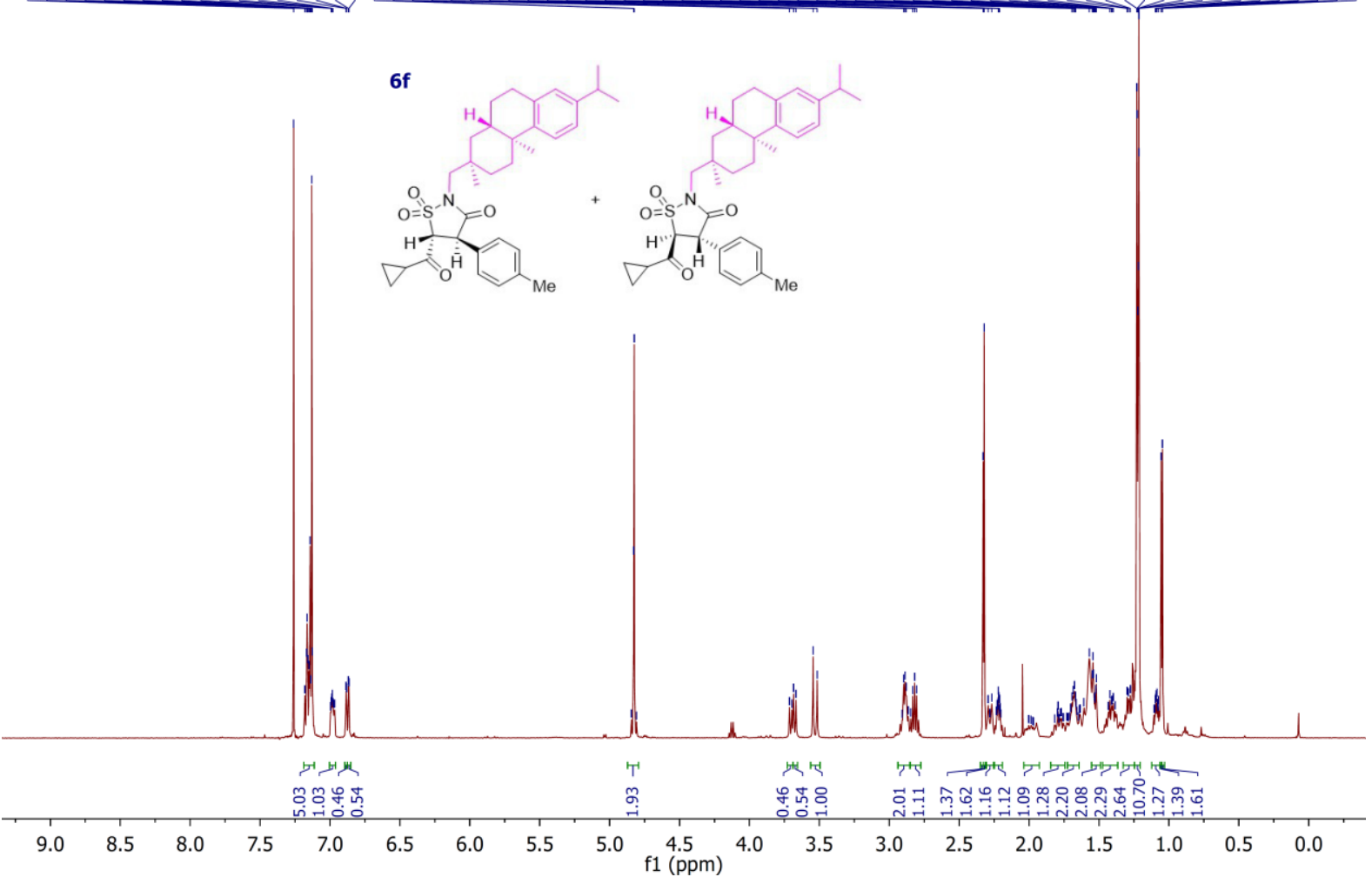

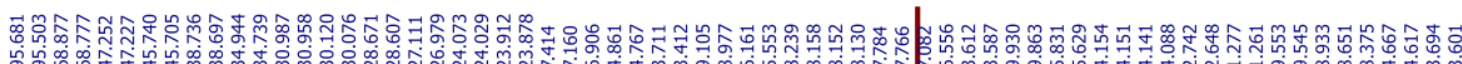

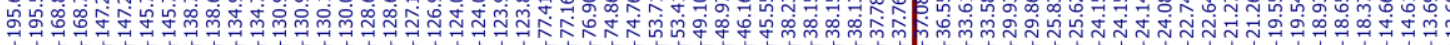
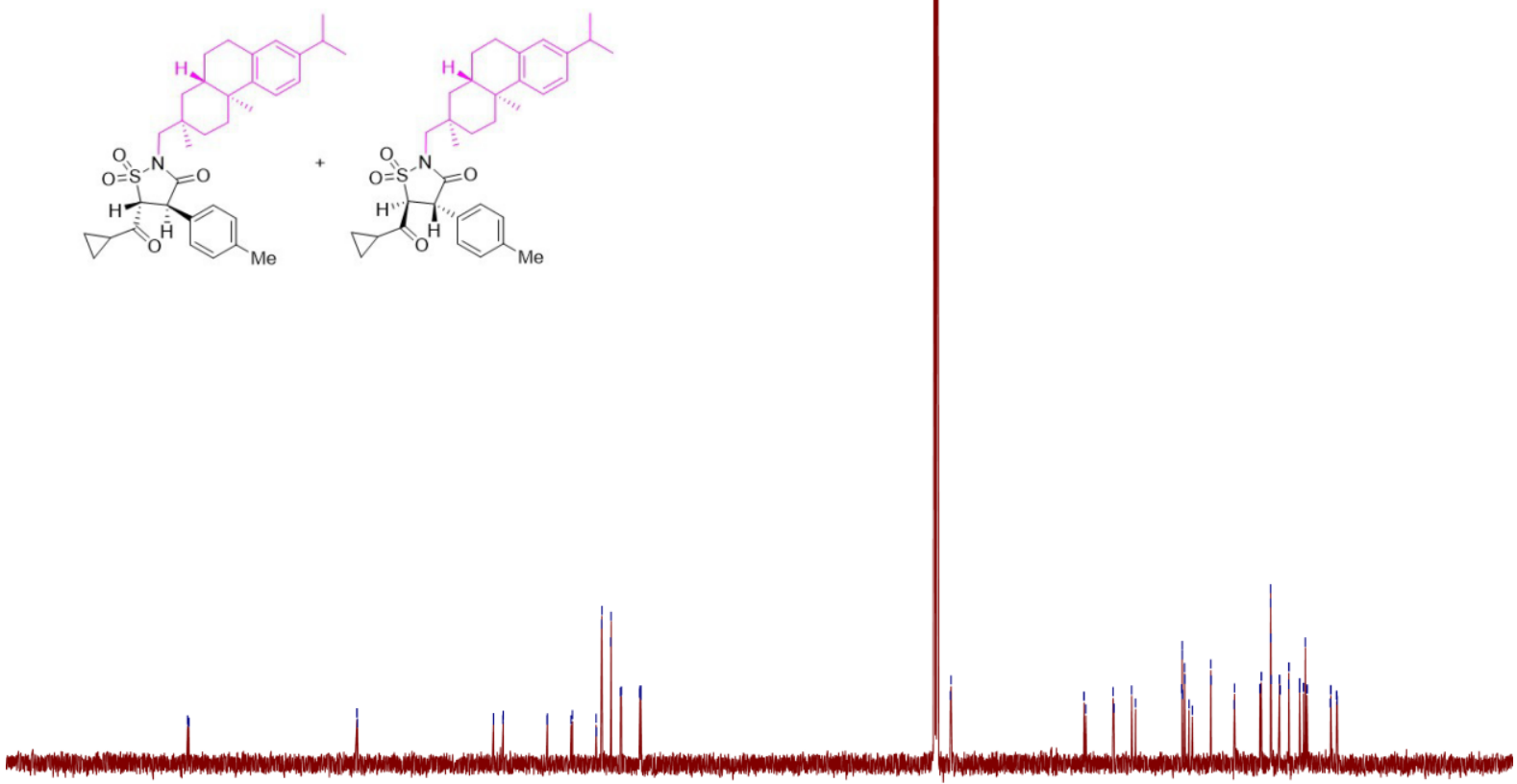

$\begin{array}{llllllllllllllllllllllll}220 & 210 & 200 & 190 & 180 & 170 & 160 & 150 & 140 & 130 & 120 & \begin{array}{l}110 \\ \mathrm{f} 1(\mathrm{ppm})\end{array} & 100 & 80 & 70 & 60 & 50 & 40 & 30 & 20 & 10 & 0 & -10\end{array}$ 

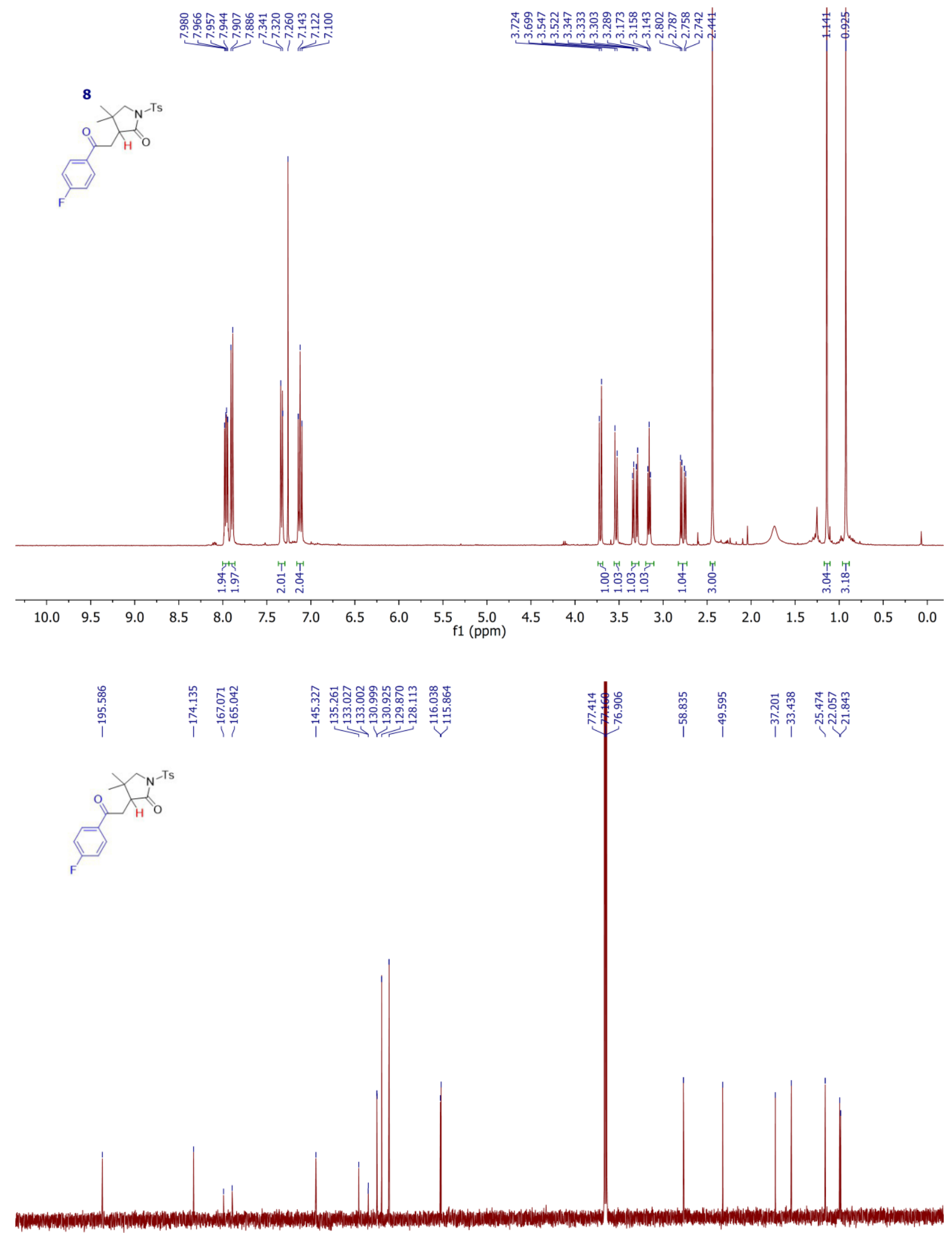

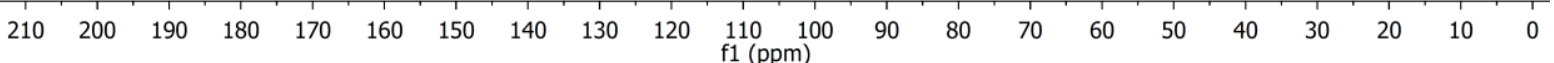



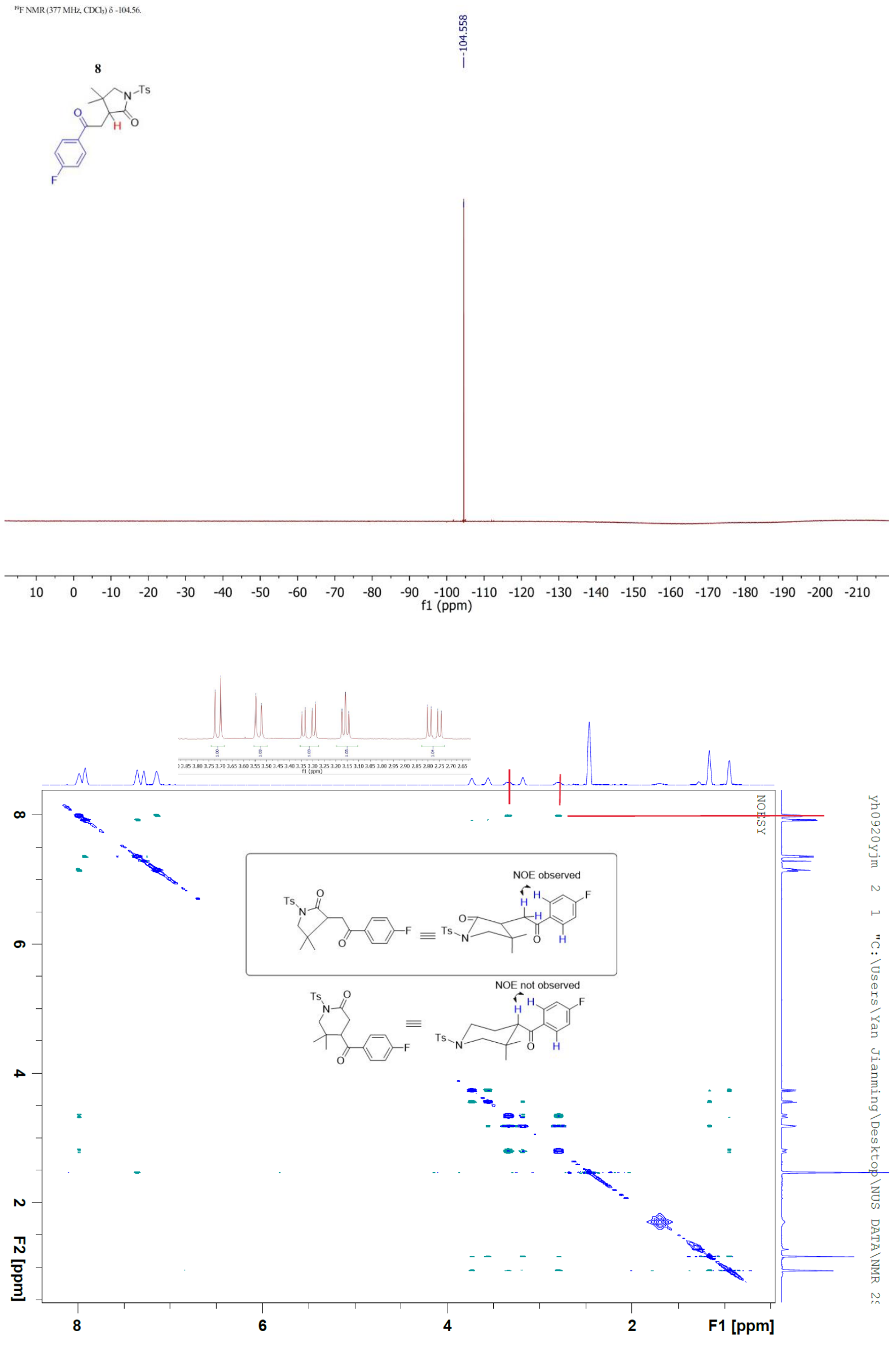


\section{References}

${ }^{1}$ West, T. H.; Walden, D. M.; Taylor, J. E.; Brueckner, A. C.; Johnston, R. C.; Cheong, P. H.-Y.; Lloyd-Jones, G. C.; Smith, A. D. J. Am. Chem. Soc. 2017, 139, 4366-4375.

${ }^{2}$ Ke, J.; Tang, Y.; Yi, H.; Li, Y.; Cheng, Y.; Liu, C.; Li, A. Angew. Chem., Int. Ed. 2015, 54, 6604-6607.

${ }^{3}$ Nicholas A. E.; Peter D. M.; Martha D. M.; Gabriel F. Synlett. 2011, 5, 699-701.

${ }^{4}$ Mukherjee, S.; Patra, T.; Glorius, F. ACS Catal. 2018, 87, 5842-5846.

${ }^{5}$ Pearson, M. S. M.; Carbery, D. R. J. Org. Chem. 2009, 74, 5320-5325.

${ }^{6}$ Narayan, A. R. H.; Jiménez-Osés, G.; Liu, P.; Negretti, S.; Zhao, W.; Ramabhadr, R. O.; Furan, L.; Li, Z.; Yang, Y.-F.; Gilbert, M.; Podust, L. M.; Montgomery, J.; Houk, K. N.; Sherman, D. H. Nature Chem. 2015, 7, 653-660.

${ }^{7}$ For the formation of a ketone as an adduct from acyl radical and BHT, see: Tan, H.; Li, H.; Ji, W.; Wang, L. Angew. Chem., Int. Ed. 2015, 54, 8374-8377.

${ }^{8}$ Ryu, I.; Tani, A.; Fukuyama, T.; Ravelli, D.; Fagnoni, M.; Albini, A. Angew. Chem., Int. Ed. 2011, 50, 1869-1872.

${ }^{9}$ Zhao, G.; Wang, T. Angew. Chem. Int. Ed. 2018, 57, 6120-6124.

${ }^{10}$ Cismesia, M. A.; Yoon, T. P. Chem. Sci. 2015, 6, 5426-5434.

${ }^{11}$ Hatchard, C. G.; Parker, C. A. Proc. Roy. Soc. (London) 1956, A235, 518-536.

${ }^{12}$ Grimme, S.; Antony, J.; Ehrlich, S.; Krieg, H. J. Chem. Phys. 2010, 132, 154104.

13 Grimme, S.; Ehrlich, S.; Goerigk, L. J. Comput. Chem. 2011, 32, 1456-1465.

${ }^{14}$ Frisch, M. J.; Trucks, G. W.; Schlegel, H. B.; Scuseria, G. E.; Robb, M. A.; Cheeseman, J. R.; Scalmani, G.; Barone, V.; Petersson, G. A.; Nakatsuji, H.; Li, X.; Caricato, M.; Marenich, A. V.; Bloino, J.; Janesko, B. G.; Gomperts, R.; Mennucci, B.; Hratchian, H. P.; Ortiz, J. V.; Izmaylov, A. F.; Sonnenberg, J. L.; Williams-Young, D.; Ding, F.; Lipparini, F.; Egidi, F.; Goings, J.; Peng, B.; Petrone, A.; Henderson, T.; Ranasinghe, D.; Zakrzewski, V. G.; Gao, J.; Rega, N.; Zheng, G.; Liang, W.; Hada, M.; Ehara, M.; Toyota, K.; Fukuda, R.; Hasegawa, J.; Ishida, M.; Nakajima, T.; Honda, Y.; Kitao, O.; Nakai, H.; Vreven, T.; Throssell, K.; Montgomery, J. A.; Peralta, J. E.; Ogliaro, F.; Bearpark, M. J.; Heyd, J. J.; Brothers, E. N.; Kudin, K. N.; Staroverov, V. N.; Keith, T. A.; Kobayashi, R.; Normand, J.; Raghavachari, K.; Rendell, A. P.; Burant, J. C.; Iyengar, S. S.; Tomasi, J.; Cossi, M.; Millam, J. M.; Klene, M.; Adamo, C.; Cammi, R.; Ochterski, J. W.; Martin, R. L.; Morokuma, K.; Farkas, O.; Foresman, J. B.; Fox, D. J. Gaussian 16, Revision A.03; Gaussian, Inc.: Wallingford, CT, 2016. 